SLAC-R-1019

\title{
Study of $|\mathrm{Vtd} / \mathrm{Vts}|$ Using a Sum of Exclusive B \to X Gamma Final States Reconstructed with the BaBar Detector
}

\author{
by \\ Mark James Tibbetts
}

June 2010 
IMPERIAL COLLEGE, UNIVERSITY OF LONDON

\title{
Study of $\left|V_{t d} / V_{t s}\right|$ Using a Sum of Exclusive $B \rightarrow X \gamma$ Final States Reconstructed with the BABAR Detector
}

\author{
by \\ Mark James Tibbetts \\ A thesis submitted in partial fulfillment for the \\ degree of Doctor of Philosophy \\ in the \\ School of Natural Sciences \\ Department of Physics
}

June 2010 


\section{Abstract}

Experimental data collected with the BABAR detector consisting of $470.9 \pm 2.8$ million $B \bar{B}$ events are used to measure the sum of seven exclusive $B \rightarrow X_{d(s)} \gamma$ transitions, where $X_{d(s)}$ is any non-strange (strange) charmless hadronic state. For each transition flavour, measurements are made in the hadronic mass ranges $0.5 \leq m_{X}<1.0 \mathrm{GeV} / c^{2}$ and $1.0 \leq$ $m_{X} \leq 2.0 \mathrm{GeV} / c^{2}$. These are extrapolated and combined in a model-dependent way to obtain the ratio of the total branching fractions, $\mathcal{B}\left(B \rightarrow X_{d} \gamma\right) / \mathcal{B}\left(B \rightarrow X_{s} \gamma\right)=0.0456 \pm$ $0.0110 \pm 0.0097$ where the first error is statistical and the second error is systematic. This is interpreted as a measurement of the ratio of CKM matrix elements $\left|V_{t d} / V_{t s}\right|=$ $0.211 \pm 0.023 \pm 0.022 \pm 0.001$ where the final error is due to theoretical uncertainty. 


\section{Acknowledgements}

Firstly I thank my supervisor, Paul Dauncey. Under his watchful eye I have matured in the last four years from a new graduate with a talent for parroting answers in exams to something more akin to a real scientific researcher. His patience with me during this transition cannot be understated. Neither can the importance of his clarity of thinking and direct critiques while discussing work I have presented to him; even when separated by eight time zones. Paul is an excellent supervisor and I am honoured to have worked with him.

The work presented in these pages would not exist without the committed work of Debbie Bard. Her dedication to this analysis before my joining the BABAR Collaboration and her valuable assistance after I took on the task of updating her work has been essential to its successful completion. It has been a pleasure working with a colleague as good natured and positive as Debbie. I am also grateful to her for introducing me to the Burning Man Festival, an experience I will never forget!

Many members of the BABAR Collaboration have provided sagely advice as I have developed this analysis. In particular I thank Bruce Schumm for his weekly inputs which have been invaluable. I am also grateful to the members of the Radiative Penguin AWG for their regular counsel, especially the conveners John Walsh, Kevin Flood and Jurgen Kroseberg. On the detector operations side I would like to show my appreciation to the members of the Trigger group: Dan Walker for teaching me the workings of the EMT and the system managers Su Dong and Rainer Bartoldus for their advice at operations meetings. Also thanks to Tom Latham for making the EMT a straightforward system to watch over!

California is a fantastic place to live and SLAC a great place to work. My moving there was a smooth process thanks to the UK liaison Jean as well as Robyn and Elaine in the International Office. The Californian experience was of course enhanced massively by the great bunch of people I spent my time with out there, either climbing volcanoes or getting drunk in downtown Palo Alto on 'Swanky Nights'. So a big thanks to Aidan, Christina, Chukwudi, David, Manny, Euginia, Graham, Jen, Jon, Kim, Matt, Mike, Rob, Sudan and Tim. I am beholden to Simon Keenan for his visit, accompanying me to Coachella to witness probably the best festival lineup ever. The trip to Alcatraz was pretty funny too. A particular shout out also goes to Will Panduro Vazquez who not only introduced me to the bay area and its fine eateries, but who has also always been available on short notice for a swift half in London to mutually lament over UK physics funding. 
I am particularly grateful to Julia Sedgbeer and Gavin Davies for giving me the opportunity to study with the Imperial HEP group and to Peter Dornan for assigning me to such a great experiment. It's also important to mention my fellow Imperial students, Andy, Chris, the two Davids, Kostas, Joe, Mark and Theo.

While finishing this thesis I have had the pleasure of living with Ali and Matin at 'The Keep' and had the support of many good friends. Thanks to Aidan, Andy, Angelo, Charlotte, Chloe, Dave, Ed (for nodding politely and staring blankly), Eden, Jon, Loz, Matt, Mike, Noemie, Paul, Phil, Rhodri, Rich (for allegedly proof reading), Rosie, Yolanna and anyone else I have forgotton.

A special acknowledgement should go to the UK tax payer (via STFC) for continuing to fund such an important area of research and for paying for my studentship in particular. Despite recent difficulties, the UK is a great place to study particle physics and the interest and support of members of the public in this research is a great motivation for those of us that carry it out.

Finally I would like to thank my family: my mother Joyce, my father Peter and my sister Emma as well as the extended Tibbetts and James families. Claire gets a special mention for joining Mum and Emma in visiting me in California; the road trip to the Grand Canyon will always be one of my fondest memories. I dedicate this thesis to my parents in the hope it partially repays the debt of your time, love and support over the past 28 years. 


\section{Declaration}

This thesis has been written entirely by the author while in candidature for a research degree at Imperial College. Where arguments and work presented rely on the work of others this has been acknowledged through references to the relevant literature.

With the exception of the coordinate transformation following equation (1.62), which has been calculated by the author, chapter 1 represents the authors best understanding of the relevant physical theory and does not present original work. Where plots have been taken from the existing literature this has been indicated.

The experiment description of chapter 2 represents the authors best understanding of the detector operations environment and does not contain any original work. All plots in this chapter have been provided by the relevant working groups of the BABAR collaboration.

The physics analysis presented from chapter 3 onwards has been performed predominantly by the author. It updates an existing physics analysis which is published in [1]. Much of the code used for event reconstruction has been developed by members of the $B A B A R$ collaboration and implemented by the author based on the previous analysis. Where any part of the analysis relies on the work of others this has been indicated in the text and references to the relevant literature provided.

Work in this thesis uses software tools developed by the BABAR collaboration which are not currently documented publicly. Such tools are described in internal BABAR Analysis Documents (BADs) which are referenced in this thesis. Furthermore published BABAR analyses are generally documented in more detail in support BADs, therefore references to published $B A B A R$ analyses also contain a support BAD number for completeness. It is likely that the BABAR collaboration will make all BADs publically available in the future.

Signed:

Date: 
"Y'know, it's very hard to talk quantum using a language originally designed to tell other monkeys where the ripe fruit is."

Terry Pratchett 


\section{Contents}

Abstract

$\begin{array}{ll}\text { Acknowledgements } & \text { ii }\end{array}$

Declaration

List of Figures $\quad$ x

List of Tables $\quad$ xiv

1 Physics Background $\quad 1$

1.1 The Standard Model of Particle Physics . . . . . . . . . . . . . . . . 1

1.1.1 Electroweak Interactions . . . . . . . . . . . . . . . . . . 3

1.1.2 Charged Current Weak Interactions of Quarks . . . . . . . . . 4

1.1.2.1 The Cabibbo-Kobayashi-Maskawa Matrix . . . . . . 5

1.1.2.2 CP Violation . . . . . . . . . . . . . . 7

1.1.2.3 The Unitarity Triangle . . . . . . . . . . . . . . 8

1.2 Phenomenology of $B \rightarrow X_{s / d} \gamma$ Decays . . . . . . . . . . . . . 9

1.2.1 Operator Product Expansion ．... . . . . . . . . . . . . . 10

1.2.1.1 Heavy Quark Expansion . . . . . . . . . . . . . . 14

1.2.1.2 Wilson Coefficient Evaluation ........... . . 14

1.2.2 Calculation of $\left|V_{t d} / V_{t s}\right| \ldots \ldots \ldots$. . . . . . . . . . . 15

1.2.2.1 Decay Rates in $B \rightarrow X \gamma \ldots \ldots \ldots \ldots$

1.2.2.2 $\left|V_{t d} / V_{t s}\right|$ from Inclusive Decays . . . . . . . . . . 16

1.2.2.3 $\left|V_{t d} / V_{t s}\right|$ from Exclusive Resonant $B \rightarrow X_{s / d} \gamma$ Decays . 19

1.2.3 Kagan-Neubert Model of Photon Energy Spectrum . . . . . . . 19

1.2.3.1 Photon Energy Spectrum . . . . . . . . . . . . 20

1.2.3.2 Hadronic Mass Spectrum . . . . . . . . . . . . . . . 21

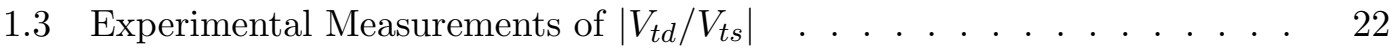

1.3.1 Measurement from $B$ Mixing . . . . . . . . . . . . . . . 22

1.3.2 Measurements from Radiative Penguin Decays . . . . . . . . . 23

1.3.3 Summary of $\left|V_{t d} / V_{t s}\right|$ Measurements . . . . . . . . . . . 23

2 The BABAR Experiment $\quad 25$ 
$2.1 \quad$ PEP-II Asymmetric $e^{+} e^{-}$Collider . . . . . . . . . . . . . . . . 26

2.2 The BABAR Detector . . . . . . . . . . . . . . . . . . . . . 28

2.2 .1 Design Goals . . . . . . . . . . . . . . . . . . 30

2.2.2 Detection of Charged Particles . . . . . . . . . . . . . . 30

2.2.2.1 The Silicon Vertex Tracker . . . . . . . . . . . . 31

2.2.2.2 The Wire Drift Chamber . . . . . . . . . . . . . 32

2.2.2.3 Charged Particle Trajectory Reconstruction . . . . . . 34

2.2 .3 Charged Hadron Identification . . . . . . . . . . . . . . 35

2.2.3.1 The Cherenkov Detector . . . . . . . . . . . 37

2.2 .4 Photon Detection . . . . . . . . . . . . . . . . 39

2.2.4.1 The Electromagnetic Calorimeter . . . . . . . . 39

2.2.4.2 Calorimeter Resolution . . . . . . . . . . . . . . 41

2.2.4.3 Calorimeter Energy Clusters . . . . . . . . . . . . 42

2.2.5 Electron and Muon Identification . . . . . . . . . . . . . . . 43

2.2.5.1 The Instrumented Flux Return . . . . . . . . . . . . . 44

2.2.6 The Trigger and Data Acquisition System . . . . . . . . . . . . 46

2.2.6.1 The Hardware Trigger . . . . . . . . . . . . . . . . 47

2.2.6.2 The Software Trigger . . . . . . . . . . . . . . . . 48

3 Event Selection $\quad 49$

3.1 Analysis Overview . . . . . . . . . . . . . . . . . . . . . . . . 49

3.1.1 Simulated and Experimental Data Samples . . . . . . . . . . 51

3.2 Event Reconstruction Framework . . . . . . . . . . . . . . . . . 53

3.2.1 Charged Tracks . . . . . . . . . . . . . . . 54

3.2.2 Photons ............................ 54

$3.2 .3 \pi^{0}$ and $\eta$ Mesons . . . . . . . . . . . . . . . . . . . . . . . 54

3.2.4 Vertex Fitting . . . . . . . . . . . . . . . . . 55

3.2.5 Charged Particle Identification Classifiers . . . . . . . . . . 55

3.2 .6 Primary Vertex . . . . . . . . . . . . . . . . . . . 56

3.3 Discriminating Variables . . . . . . . . . . . . . . . . 56

3.3.1 Kinematic Variables . . . . . . . . . . . . . . . . . 57

3.3.2 Topological Variables . . . . . . . . . . . . . . . . . . . 59

3.3.2.1 Thrust . . . . . . . . . . . . . . . 60

3.3.2.2 Energy and Momentum Flow . . . . . . . . . . 61

3.3.2.3 Sphericity Tensor . . . . . . . . . . . . . . . . . 62

3.3.2.4 Angular Momentum . . . . . . . . . . . . . . . 63

3.3.3 Flavour Tagging Variables . . . . . . . . . . . . . . . . 64

3.3.3.1 Lepton Tagging . . . . . . . . . . . . . . 64

3.3.3.2 Hadron Tagging . . . . . . . . . . . . . . . . . 67

3.3.3.3 Momentum Tagging . . . . . . . . . . . . . . . 69

3.4 Event Reduction Filters . . . . . . . . . . . . . . . . . . . . . 70

3.4.1 BToXdGammaFilter ................. . . . . . . . 70

3.4 .2 Filter Efficiencies . . . . . . . . . . . . . . . . . . 73

3.5 Full Event Reconstruction . . . . . . . . . . . . . . . . . . . . . 73

3.5.1 Signal Candidate Identification . . . . . . . . . . . 75

3.6 Post Reconstruction Event Reduction . . . . . . . . . . . . . . . . . . 77

3.6.1 $B \rightarrow X_{s} \gamma$ Background from $K_{S}^{0}$ Decays . . . . . . . . . 77 
3.6.2 High Energy Photon Candidate . . . . . . . . . . . . . . . . 79

3.6.3 $X_{s / d}$ Candidate Cuts . . . . . . . . . . . . . . . . 82

3.6.4 B Meson Candidate Cuts . . . . . . . . . . . . . . . . . . 83

3.6.4.1 Hadronic Mass Bin Variations . . . . . . . . . . . . 83

3.6.5 Multivariate Classifier for Continuum Event Reduction . . . . . 84

3.6.5.1 Overview of Neural Networks . . . . . . . . . 85

3.6.5.2 Classification of Training and Testing Events . . . . . 86

3.6.5.3 Training Strategy and Input Variable Selection . . . . 88

3.6.6 Best Candidate Selection . . . . . . . . . . . . . . . . . 89

3.6.7 Cut Optimisation . . . . . . . . . . . . . . . . . . . . 90

3.6.7.1 Discussion of Analysis Strategy . . . . . . . . . . 91

3.7 Event Selection Summary . . . . . . . . . . . . . . . . . . 93

3.7.1 Estimated Data Yields and Selection Efficiencies . . . . . . . . 94

$\begin{array}{llr}4 & \text { Signal Yield Extraction } & 100\end{array}$

4.1 Maximum Likelihood . . . . . . . . . . . . . . . . . . . . . 100

4.1 .1 Overview .......................... 100

4.1.2 Extended Maximum Likelihood . . . . . . . . . . . . . . 101

$4.1 .3 \quad$ Fit Variables . . . . . . . . . . . . . . . . . . . . . . . . . 102

4.2 Corrections to $B \rightarrow X \gamma \mathrm{MC}$ data . . . . . . . . . . . . . . . . 102

4.3 Event Hypotheses ． . . . . . . . . . . . . . . . . . . . . . . . . . . . 104

4.3.1 $B \rightarrow X_{s} \gamma$ Candidate Events . . . . . . . . . . . . . . 104

4.3.2 $B \rightarrow X_{d} \gamma$ Candidate Events . . . . . . . . . . . . . 105

4.4 PDF Parameterisation . . . . . . . . . . . . . . . . . . 105

4.4.1 PDF Functional Forms . . . . . . . . . . . . . . . . . . 105

4.4.2 $\quad B \rightarrow X_{s} \gamma$ Candidate PDFs . . . . . . . . . . . . . . 111

4.4.3 $B \rightarrow X_{d} \gamma$ Candidate PDFs . . . . . . . . . . . . . 116

4.5 Combined Fit Strategy . . . . . . . . . . . . . . . . . . . 116

4.5.1 Combined Fit Overview . . . . . . . . . . . . . . . . . 122

4.5.2 Studies to Test for Fit Stability and Bias . . . . . . . . . . . . 123

4.5.2.1 $X_{s}$ Candidate Fits . . . . . . . . . . . . . . . 125

4.5.2.2 $X_{d}$ Candidates . . . . . . . . . . . . . 125

4.6 Fits To Experimental Data . . . . . . . . . . . . . . . . . . . . 129

4.6.1 $X_{s}$ Candidate Fit Results . . . . . . . . . . . . . . . . . 129

$4.6 .2 \quad X_{d}$ Candidate Fit Results . . . . . . . . . . . . . . . . . . . . 132

4.6.3 Quality of Fits to Experimental Data . . . . . . . . . . . . 134

4.6.4 Interpretation of Results . . . . . . . . . . . . . . . . 137

5 Post Fit Studies $\quad 139$

5.1 Experimental Systematic Uncertainties . . . . . . . . . . . . . . . 139

5.1.1 Reconstruction Efficiencies . . . . . . . . . . . . . . . . 139

5.1.1.1 Charged Tracks . . . . . . . . . . . . . . . 140

5.1.1.2 Neutral Particles . . . . . . . . . . . . . . . . . . . 141

5.1.2 Event Reduction Cut Efficiencies . . . . . . . . . . . . . 141

5.1.2.1 Photon Cuts . . . . . . . . . . . . . . . 142

5.1.2.2 Charged Track PID . . . . . . . . . . . . . . . 142

5.1.2.3 Hadronic Candidate and Event Topology Cuts . . . . . 144 
5.1.3 Fit Uncertainties . . . . . . . . . . . . . . . . . 150

5.1.3.1 Fixed PDF Parameters . . . . . . . . . . . . . 150

5.1.3.2 Agreement Between MC and Experimental Data . . . 151

5.1.3.3 Fixed Event Class Normalisations . . . . . . . . . . . 154

5.1 .3 .4 Fit Bias . . . . . . . . . . . . . . . . . 155

5.1.4 Signal Model Uncertainties . . . . . . . . . . . . . . . . . 156

5.1.4.1 Photon Spectrum Model . . . . . . . . . . . 156

5.1.4.2 JETSET Phase Space Model . . . . . . . . . . . . . . 158

5.1.4.3 Resonant Final States . . . . . . . . . . . . . . 160

5.1.5 Summary of Experimental Systematic Uncertainties . . . . . . . 161

5.2 Measured BF Extrapolation and Extraction of $\left|V_{t d} / V_{t s}\right| \ldots \ldots$. . . . . 162

5.2.1 Low Mass Region BF Extrapolation . . . . . . . . . . . . . . . 162

5.2.2 High Mass Region BF Extrapolation . . . . . . . . . . . . . . 164

5.2.2.1 Correction for Final States with $1.0 \leq m_{X} \leq 2.0 \mathrm{GeV} / c^{2} \quad 164$

5.2.2.2 Correction for Final States with $m_{X}>2.0 \mathrm{GeV} / c^{2} \ldots . \quad 167$

5.2.2.3 Summary of Extrapolated BFs . . . . . . . . . . 167

5.2.3 Calculation of $\left|V_{t d} / V_{t s}\right|$ and Associated Uncertainty . . . . . . 168

6 Summary and Future Prospects 171

6.1 Future Prospects . . . . . . . . . . . . . . . . . . . . . . . 173

6.2 Addendum . . . . . . . . . . . . . . . . . . . . . 174

A Monomial Functions and Sphericity Tensor Variable Distributions $\mathbf{1 7 5}$

A.1 Monomial Function Distributions . . . . . . . . . . . . . . . . 175

A.2 Sphericity Tensor Variable Distributions . . . . . . . . . . . . . 182

$\begin{array}{lr}\text { B Signal Monte Carlo Data Event Classification } & 184\end{array}$

B.1 Resonant MC data . . . . . . . . . . . . . . . . . . . . . . 184

B.1.1 $B \rightarrow X_{s} \gamma \ldots \ldots \ldots \ldots \ldots \ldots \ldots$

B.1.2 $B \rightarrow X_{d} \gamma \ldots \ldots \ldots \ldots \ldots$. . . . . . . . . . . . . . . . . . . . . . . .

B.2 Non-Resonant MC data with Generated Hadronic Mass Following the KN

Model . . . . . . . . . . . . . . . . . . 185

B.2.1 $B \rightarrow X_{s} \gamma \ldots \ldots \ldots \ldots \ldots \ldots$

B.2.2 $B \rightarrow X_{d} \gamma \ldots \ldots \ldots \ldots$. . . . . . . . . . . 186

$\begin{array}{lr}\text { References } & 190\end{array}$ 


\section{List of Figures}

1.1 The Unitarity Triangle . . . . . . . . . . . . . . . . . . . . . 8

1.2 Standard Model Feynman Diagram for the Flavour Changing Neutral Current $b \rightarrow s / d \gamma \ldots \ldots$. . . . . . . . . . . . . . . . . 9

1.3 Possible supersymmetry contributions to $b \rightarrow s / d \gamma \ldots \ldots$

1.4 Tree level weak transition $b \rightarrow u d \bar{u} \ldots$. . . . . . . . . . . . . . . . . 11

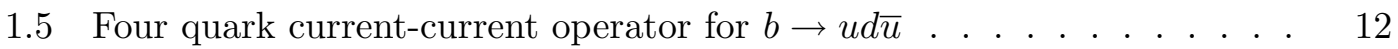

1.6 QCD corrections to $b \rightarrow u d \bar{u} \ldots \ldots \ldots$. . . . . . . . . . 12

1.7 QCD penguin operators . . . . . . . . . . . . . . . . . 13

1.8 Magnetic penguin operators . . . . . . . . . . . . . . . . . . 13

1.9 Calculated variation of $\left|V_{t d} / V_{t s}\right|$ as a function of $R(d \gamma / s \gamma) \ldots \ldots$

1.10 Weak annihilation in the process $B^{-} \rightarrow \rho^{-} \gamma \ldots \ldots$. . . . . . . . 19

$1.11 \mathrm{KN}$ model photon spectra for various values of parameters $\left(m_{b}, \mu_{\pi}^{2}\right)$. . 20

$1.12 \mathrm{KN}$ model mass spectra for various values of parameter $m_{b}$. . . . . . 22

1.13 Dominant Feynman diagram contributing to neutral $B$ meson mixing . 23

2.1 The PEP-II Storage Rings _ . . . . . . . . . . . . . . . . . 26

2.2 Plot of BABAR Recorded Luminosity . . . . . . . . . . . . . . . . . 27

2.3 The BABAR Detector in Longitudinal and Transverse Section . . . . . . 29

2.4 The SVT in Longitudinal and Transverse Section . . . . . . . . . . . . 31

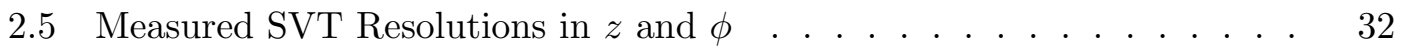

2.6 DCH Superlayer Structure . . . . . . . . . . . . . . . . . . . 33

2.7 Transverse DCH Cell Resolution . . . . . . . . . . . . . . . . . 34

2.8 Resolution in Transverse Momentum of the SVT and DCH combined . 35

2.9 Bethe-Bloch Curves Compared to Data for Different Particle Species in the $\mathrm{DCH} \ldots \ldots \ldots \ldots$. . . . . . . . . . . . . . 36

2.10 Longitudinal Section of the DIRC . . . . . . . . . . . . . . 37

2.11 Schematic Showing Principle of DIRC Operation . . . . . . . . . . . . 38

2.12 Transverse Section of a Side of the DIRC Barrel . . . . . . . . . . . . 38

2.13 Plots to Illustrate DIRC Performance . . . . . . . . . . . . . . . . . 39

2.14 Longitudinal Section of the EMC . . . . . . . . . . . . . . . . 40

2.15 Schematic of a $\mathrm{CsI}(\mathrm{Tl})$ Crystal . . . . . . . . . . . . . . . . . . . . . 40

2.16 Plot of EMC Energy Resolution in Data . . . . . . . . . . . . . . . 41

2.17 Plot of EMC Angular Resolution for $\pi^{0} \rightarrow \gamma \gamma$ Decays . . . . . . . . . 42

2.18 Layout of the IFR . . . . . . . . . . . . . . . . . . . . . . . . . . . . . . . . 44

2.19 Schematic Showing RPC Design . . . . . . . . . . . . . . . . . . 45

2.20 Sketch of an LST in Transverse Section . . . . . . . . . . . . . . 45

2.21 Comparison of IFR Efficiency for High Energy Muons . . . . . . . . . 46 
3.1 Schematic representation of the primary vertex in a $B \bar{B}$ event. . . . . .

3.2 Distributions of $m_{E S}^{\prime}$ for continuum and correctly reconstructed candidates before post-reconstruction cuts. . . . . . . . . . . . .

3.3 Distributions of $\Delta E$ for continuum and correctly reconstructed candidates before post-reconstruction cuts. . . . . . . . . . . . . .

3.4 Distributions of $T_{R O E}$ for continuum and correctly reconstructed candidates before post-reconstruction cuts. . . . . . . . . . . .

3.5 Distributions of $\left|\cos \theta_{T}\right|$ for continuum and correctly reconstructed candidates before post-reconstruction cuts. . . . . . . . . . . . .

3.6 Distributions of $R_{2}^{\prime}$ for continuum and correctly reconstructed candidates before post-reconstruction cuts. . . . . . . . . . . . . .

3.7 Distributions of $\left|\cos \theta_{B}\right|$ for continuum and correctly reconstructed candidates before post-reconstruction cuts. . . . . . . . . . .

3.8 Distributions of $L_{T A G}^{e}$ for continuum and correctly reconstructed candidates before post-reconstruction cuts. . . . . . . . . . . .

3.9 Distributions of $L_{T A G}^{\mu}$ for continuum and correctly reconstructed candidates before post-reconstruction cuts. . . . . . . . . . . . .

3.10 Distributions of $L_{T A G}^{l}$ for continuum and correctly reconstructed candidates before post-reconstruction cuts. . . . . . . . . . . . .

3.11 Distributions of $H_{T A G}^{\pi}$ for continuum and correctly reconstructed candidates before post-reconstruction cuts. . . . . . . . . . . . .

3.12 Distributions of $H_{T A G}^{K}$ for continuum and correctly reconstructed candidates before post-reconstruction cuts. . . . . . . . . . . . . .

3.13 Distributions of $H_{T A G}^{K \pi}$ for continuum and correctly reconstructed candidates before post-reconstruction cuts. . . . . . . . . . . . . .

3.14 Distributions of $P_{T A G}$ for continuum and correctly reconstructed candidates before post-reconstruction cuts. . . . . . . . . . . .

3.15 Distribution of $\Delta m_{X}$ for signal MC data candidates. . . . . . . . . . . 76

3.16 Distance between decay vertex and event primary vertex for reconstructed $X_{d}$ candidates in the high mass region. . . . . . . . . . . 78

3.17 Invariant mass distribution of reconstructed $K_{S}^{0} \rightarrow \pi^{+} \pi^{-}$candidates. . . 78

3.18 Distributions of best $\pi^{0}$ mass for continuum and correctly reconstructed candidates before post-reconstruction cuts. . . . . . . . . . . .

3.19 Distributions of best $\eta$ mass for continuum and correctly reconstructed candidates before post-reconstruction cuts. . . . . . . . . . . . .

3.20 Distributions of $S_{\text {bump }}^{\prime}$ for continuum and correctly reconstructed candidates before post reconstruction cuts. . . . . . . . . . . . . . . . 81

3.21 Distributions of the momentum magnitude of the lowest momentum hadronic daughter for continuum and correctly reconstructed candidates before post reconstruction cuts.

3.22 Schematic Representation of a Neural Network . . . . . . . . . . . . . 85

3.23 Distributions of the NN output for continuum and correctly reconstructed candidates. . . . . . . . . . . . . . . . . .

4.1 2D plots of $m_{E S}^{\prime}$ and $\Delta E$ for $B \rightarrow X_{s}^{L} \gamma$ candidates . . . . . . . . . 106

4.2 2D plots of $m_{E S}^{\prime}$ and $\Delta E$ for $B \rightarrow X_{s}^{H} \gamma$ candidates . . . . . . . . 107

4.32 D plots of $m_{E S}^{\prime}$ and $\Delta E$ for $B \rightarrow X_{d}^{L} \gamma$ candidates . . . . . . . . . . 108

4.4 2D plots of $m_{E S}^{\prime}$ and $\Delta E$ for $B \rightarrow X_{d}^{H} \gamma$ candidates . . . . . . . . . 109 


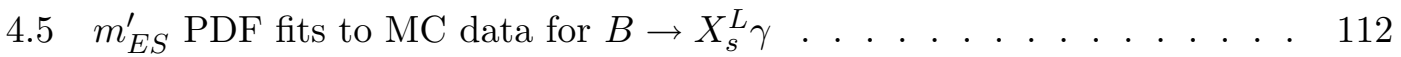

4.6 $\Delta E \mathrm{PDF}$ fits to $\mathrm{MC}$ data for $B \rightarrow X_{s}^{L} \gamma$ candidates . . . . . . . . . . 113

$4.7 m_{E S}^{\prime}$ PDF fits to MC data for $B \rightarrow X_{s}^{H} \gamma$ candidates . . . . . . . . . . 114

$4.8 \Delta E$ PDF fits to MC data for $B \rightarrow X_{s}^{H} \gamma$ candidates . . . . . . . . . . . 115

$4.9 m_{E S}^{\prime}$ PDF fits to MC data for $B \rightarrow X_{d}^{L} \gamma$ candidates . . . . . . . . . . 118

$4.10 \Delta E$ PDF fits to MC data for $B \rightarrow X_{d}^{L} \gamma$ candidates . . . . . . . . . . . 119

$4.11 m_{E S}^{\prime}$ PDF fits to MC data for $B \rightarrow X_{d}^{H} \gamma$ candidates . . . . . . . . . . 120

$4.12 \Delta E \mathrm{PDF}$ fits to $\mathrm{MC}$ data for $B \rightarrow X_{d}^{H} \gamma$ candidates . . . . . . . . . 121

4.13 Toy study signal yield pull distributions for $B \rightarrow X_{s} \gamma$ candidates fits to experimental data . . . . . . . . . . . . . . . . . . . 127

4.14 Toy study signal yield pull distributions for $B \rightarrow X_{d} \gamma$ candidates fits to experimental data . . . . . . . . . . . . . . .

4.15 Projection plots of $m_{E S}^{\prime}$ and $\Delta E$ for $B \rightarrow X_{s}^{L} \gamma$ candidate fit to experimental data . . . . . . . . . . . . . . . . . . . . 130

4.16 Projection plots of $m_{E S}^{\prime}$ and $\Delta E$ for $B \rightarrow X_{s}^{H} \gamma$ candidate fit to experimental data . . . . . . . . . . . . . . . . .

4.17 Projection plots of $m_{E S}^{\prime}$ and $\Delta E$ for $B \rightarrow X_{d}^{L} \gamma$ candidate fit to experimental data . . . . . . . . . . . . . . . .

4.18 Projection plots of $m_{E S}^{\prime}$ and $\Delta E$ for $B \rightarrow X_{d}^{H} \gamma$ candidate fit to experimental data . . . . . . . . . . . . . . . . .

5.1 Efficiency comparisons of PID selector performance between MC and experimental data control samples . . . . . . . . . . . .

5.2 Systematic variation for $n_{s i g} / \epsilon_{M C}$ as a function of $\left|p_{\text {min }}\right|$ cut in $B \rightarrow X_{s} \gamma$ data fits . . . . . . . . . . . . . . . . 146

5.3 Systematic variation for $n_{\text {sig }} / \epsilon_{M C}$ as a function of $\chi^{2}$ probability cut in $B \rightarrow X_{s} \gamma$ data fits . . . . . . . . . . . . . . . . . 147

5.4 Systematic variation for $n_{s i g} / \epsilon_{M C}$ as a function of $x_{D E C}$ cut in $B \rightarrow X_{s} \gamma$ data fits . . . . . . . . . . . . . . . . . . . . 148

5.5 Systematic variation for $n_{s i g} / \epsilon_{M C}$ as a function of NN cut in $B \rightarrow X_{s} \gamma$ data fits . . . . . . . . . . . . . . . . . . . 149

5.6 Weights applied to generated hadronic mass spectrum to emulate alternative $\mathrm{KN}$ model parameterisations.

A.1 Distributions of the monomial $L_{1}^{T} \ldots \ldots \ldots \ldots$. . . . . . . . 176

A.2 Distributions of the monomial $L_{2}^{T} \ldots \ldots \ldots \ldots$. . . . . . . 176

A.3 Distributions of the monomial $L_{3}^{T} \ldots \ldots \ldots$. . . . . . . . . . . 177

A.4 Distributions of the monomial $P_{1}^{T} \ldots \ldots \ldots \ldots$. . . . . . . . . . . . . . . . . 177

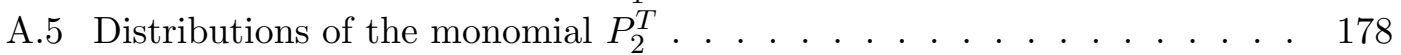

A.6 Distributions of the monomial $P_{3}^{T} \ldots \ldots \ldots \ldots$. . . . . . . . . . . . . . . . . . . . 178

A.7 Distributions of the monomial $L_{1}^{\gamma} \ldots \ldots \ldots \ldots$. . . . . . . . . . 179

A.8 Distributions of the monomial $L_{2}^{\gamma} \ldots \ldots \ldots \ldots$. . . . . . . . . . . 179

A.9 Distributions of the monomial $L_{3}^{\gamma} \ldots \ldots \ldots$. . . . . . . . . . 180

A.10 Distributions of the monomial $P_{1}^{\gamma}$. . . . . . . . . . . . . . . 180

A.11 Distributions of the monomial $P_{2}^{\gamma}$. . . . . . . . . . . . . . . 181

A.12 Distributions of the monomial $P_{2}^{\gamma} \ldots \ldots \ldots$. . . . . . . . . . . . . . . . . . . . . . . 181

A.13 Distributions of $S_{R O E} \ldots \ldots \ldots \ldots \ldots \ldots$

A.14 Distributions of $P_{R O E} \ldots \ldots \ldots \ldots$ 
A.15 Distributions of $A_{R O E} \ldots \ldots \ldots \ldots$ 


\section{List of Tables}

1.1 The quarks and leptons of the Standard Model . . . . . . . . . . . . . 2

1.2 Values of the Wolfenstein Parameters . . . . . . . . . . . . . . . . . 9

1.3 Values of input parameters for the evaluation of $R(d \gamma / s \gamma) \ldots \ldots$

3.1 Reconstructed exclusive modes . . . . . . . . . . . . . . . . . . . . 49

3.2 Simulated dataset sizes . . . . . . . . . . . . . . . . . . . . . . 52

3.3 High mass bin simulated signal sizes after model based hadronic mass cut 52

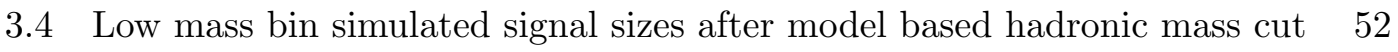

3.5 Filter efficiencies for high mass bin signal MC data . . . . . . . . . . . 71

3.6 Filter efficiencies for low mass bin signal MC data . . . . . . . . . . . 72

3.7 High mass bin signal MC samples after filter and hadronic mass cuts . . 72

3.8 Low mass bin signal MC samples after filter and hadronic mass cuts . . 73

3.9 Filter efficiencies for generic MC collections and experimental data . . . 73

3.10 Events with at least one reconstructed candidate . . . . . . . . . . . 75

3.11 Variations in the high mass region used for cut optimisation . . . . . . 84

3.12 Variations in the low mass region used for cut optimisation . . . . . . . 84

3.13 Unoptimised cut values used to assign training events . . . . . . . . . 86

3.14 Required make up of training samples to reflect expected proportions in data . . . . . . . . . . . . . . . . . 87

3.15 Size of NN training and testing samples for each optimisation class . . . 87

3.16 Variables considered for $\mathrm{NN}$ input . . . . . . . . . . . . . . . . . . 88

3.17 Cut optimisation ranges for continuous variables . . . . . . . . . . . . 91

3.18 Final FOM for each optimisation class . . . . . . . . . . . . . . . . . . 92

3.19 Optimised cut values for the high mass region . . . . . . . . . . . . 93

3.20 Optimised cut values for the low mass region . . . . . . . . . . . . . 94

$3.21 \mathrm{MC}$ data sample sizes after event selection . . . . . . . . . . . . . 95

3.22 Estimates of $X_{d}$ candidate yields and selection efficiencies . . . . . . . 96

3.23 Estimates of $X_{s}$ candidate yields and selection efficiencies . . . . . . . 97

3.24 Total efficiency by mode for correctly reconstructed high mass $X_{d}$ and $X_{s}$ candidates . . . . . . . . . . . . . . . . . . . . . 98

3.25 Total efficiency as a function of generated hadronic mass for correctly reconstructed high mass $X_{d}$ candidates . . . . . . . . . . . . . . . . 98

4.1 Corrected event yield estimates for $B \rightarrow X_{s} \gamma$ event hypotheses after MC data weighting . . . . . . . . . . . . . . . . . 103

4.2 Fitted $m_{E S}^{\prime}$ PDF parameters in MC data for low and high mass region $B \rightarrow X_{s} \gamma$ candidates. . . . . . . . . . . . . . . . . . 111 
4.3 Fitted $\Delta E$ PDF parameters in MC data for low and high mass region $B \rightarrow X_{s} \gamma$ candidates. . . . . . . . . . . . . . .

4.4 Fitted $m_{E S}^{\prime}$ PDF parameters in MC data for low and high mass region $B \rightarrow X_{d} \gamma$ candidates. . . . . . . . . . . . . .

4.5 Fitted $\Delta E$ PDF parameters in MC data for low and high mass region $B \rightarrow X_{d} \gamma$ candidates. . . . . . . . . . . . .

4.6 Summary of fixed and floated event class yields and PDF parameters in fits to experimental data . . . . . . . . . . . . . .

4.7 Fitted Gaussian parameters to pull distributions obtained from toy MC studies for the low mass $B \rightarrow X_{s} \gamma$ fit . . . . . . . . . . .

4.8 Fitted Gaussian parameters to pull distributions obtained from toy MC studies for the high mass $B \rightarrow X_{s} \gamma$ fit . . . . . . . . . .

4.9 Fitted Gaussian parameters to pull distributions obtained from toy MC studies for the low mass $B \rightarrow X_{d} \gamma$ fit . . . . . . . . . . . . . . . . 127

4.10 Fitted Gaussian parameters to pull distributions obtained from toy MC studies for the high mass $B \rightarrow X_{d} \gamma$ fit . . . . . . . . . . . . . . . 129

4.11 Result of fit to experimental data for $B \rightarrow X_{s}^{L} \gamma$ candidates . . . . . . . 132

4.12 Result of fit to experimental data for $B \rightarrow X_{s}^{H} \gamma$ candidates . . . . . . 132

4.13 Corrections to $B \rightarrow X_{s} \gamma$ cross-feed components yields in fits to $B \rightarrow X_{d} \gamma$ candidates after fitting for $B \rightarrow X_{s} \gamma$ candidates in experimental data .

4.14 Corrections to $\Delta E$ signal widths in fits to $B \rightarrow X_{d} \gamma$ candidate data after fitting for $B \rightarrow X_{s} \gamma$ candidates in experimental data . . . . . . . . . . 133

4.15 Result of fit to experimental data for $B \rightarrow X_{d}^{L} \gamma$ candidates . . . . . . . 134

4.16 Result of fit to experimental data for $B \rightarrow X_{d}^{H} \gamma$ candidates . . . . . . 134

$4.17 \chi^{2}$ goodness of fit test results for fits to experimental data . . . . . . . 137

4.18 Measured BFs for each fit to experimental data . . . . . . . . . . . . . 138

5.1 Estimated uncertainties from charged track reconstruction . . . . . . . 140

5.2 Estimated uncertainties from neutral particle reconstruction . . . . . . 141

5.3 Estimated uncertainties from PID selectors . . . . . . . . . . . . . 144

5.4 Systematic uncertainties from fixing $m_{E S}^{\prime}$ PDF parameters in fits to experimental data. . . . . . . . . . . . . . . . . . . 151

5.5 Systematic uncertainties from fixing $\Delta E$ PDF parameters in fits to experimental data. . . . . . . . . . . . . . . . . .

5.6 Systematic uncertainties from fixing event hypothesis yields in fits to experimental data. . . . . . . . . . . . . . . .

5.7 Systematic uncertainties from bias on parameters floated in fits to experimental data . . . . . . . . . . . . . . . . . . .

5.8 Fitted weight functions and calculated MC data efficiency for alternative KN parameterisations in $B \rightarrow X_{s} \gamma \mathrm{MC}$ data . . . . . . . . . . .

5.9 Fitted weight functions and calculated MC data efficiency for alternative KN parameterisations in $B \rightarrow X_{d} \gamma \mathrm{MC}$ data . . . . . . . . . .

5.10 Systematic uncertainties due to uncertainties in the weights applied to $B \rightarrow X_{s}^{H} \gamma$ signal MC data modes . . . . . . . . . . . . . . .

5.11 Comparison of final state particles when substituting the $s$ quark for a $d$

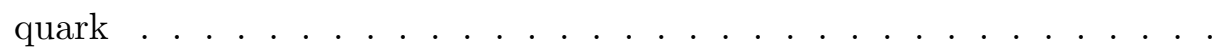

5.12 Weights assigned to $B \rightarrow X_{d}^{H} \gamma$ signal modes to obtain an alternative model of final state contributions . . . . . . . . . . . . . . . . . 
5.13 Uncertainty in signal efficiency due to assumed relative contribution of individual resonant final states . . . . . . . . . . . . . . . . 161

5.14 Estimates of total systematic uncertainty on measured BFs . . . . . . . 163

5.15 Measured BFs showing calculated systematic uncertainties before BF extrapolation. . . . . . . . . . . . . . . . . . . . 164

5.16 Systematic uncertainties due to weights applied to $B \rightarrow X_{s}^{H} \gamma \mathrm{MC}$ data after BF extrapolation . . . . . . . . . . . . . . 165

5.17 Resonant decays used for alternative fragmentation model . . . . . . . 166

5.18 Extrapolated BF summary. . . . . . . . . . . . . . . . . 168

5.19 Estimated uncertainties in the calculation of $R \ldots \ldots$

6.1 Summary of measured BFs . . . . . . . . . . . . . . . . . . . 171

6.2 Summary of extrapolated BFs . . . . . . . . . . . . . . . . 172

6.3 Existing world average BF measurements . . . . . . . . . . . . . 172

$6.4\left|V_{t d} / V_{t s}\right|$ measurement comparisons . . . . . . . . . . . . . 172

B.1 Distribution of final states in $B^{+} \rightarrow K^{*+} \gamma$ signal MC data . . . . . . . 184

B.2 Distribution of final states in $B^{0} \rightarrow K^{* 0} \gamma$ signal MC data . . . . . . 185

B.3 Distribution of final states in $B^{0} \rightarrow \omega \gamma$ signal MC data . . . . . . . . . 185

B.4 Distribution of final states in high mass $B^{+} \rightarrow X_{s}^{+} \gamma$ signal MC data . . 186

B.5 Distribution of final states in high mass $B^{0} \rightarrow X_{s}^{0} \gamma$ signal MC data . . 187

B.6 Application of weights to high mass $B \rightarrow X_{s} \gamma$ signal MC data . . . . 188

B.7 Distribution of final states in high mass $B^{+} \rightarrow X_{d}^{+} \gamma$ signal MC data . . 188

B.8 Distribution of final states in high mass $B^{0} \rightarrow X_{d}^{0} \gamma$ signal MC data . . 189 


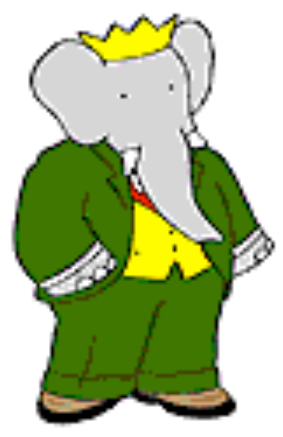

For my family. 


\section{Chapter 1}

\section{Physics Background}

This thesis presents a study of $b \rightarrow s / d \gamma$ electroweak radiative penguin decays using $e^{+} e^{-}$collision data from the BABAR experiment. Due to quark confinement the process is studied through analysis of the inclusive hadronic decays $B \rightarrow X_{s / d} \gamma$, where $X_{s / d}$ represents any charmless hadronic system formed from the resultant $s / d$ quark and spectator quark of the $B$ meson. This chapter motivates the study by discussing the relevant physical theory. An overview of electroweak interactions in the Standard Model $(\mathrm{SM})$ is given and flavour mixing in the quark sector is considered from descriptions of the Cabbibo-Kobayashi-Maskawa (CKM) matrix [2,3] and resulting Unitarity Triangle (UT) [4]. The phenomenology of inclusive $B \rightarrow X_{s / d} \gamma$ decays is discussed through an introduction to rate calculations using operator product expansion. The relation of such rates to the ratio of CKM matrix elements $\left|V_{t d} / V_{t s}\right|$ then directly motivates the analysis described in subsequent chapters. Finally modelling of the photon energy spectrum is discussed. The following overview of the relevant theory from the SM of particle physics is largely based on the text books [5-7] and the content of lecture courses attended by the author, the proceedings of which are available in $[8,9]$.

\subsection{The Standard Model of Particle Physics}

The fundamental constituents of matter, the quarks and leptons, are fermions which form three generations as shown in table 1.1. Their interactions, with the exception of gravity, are described by the Standard Model of particle physics. The SM is constructed by performing gauge transformations on the free Dirac field equation of the fermion spinors and then requiring the transformations be a local space-time symmetry of the Lagrangian. This demands a covariant derivative which, when acting on the fermion 
(a)

\begin{tabular}{|l||lll|}
\hline$Q$ & 1 & 2 & 3 \\
\hline$-\frac{1}{3}$ & $d$ & $s$ & $b$ \\
$+\frac{2}{3}$ & $u$ & $c$ & $t$ \\
\hline
\end{tabular}

(b)

\begin{tabular}{|r||lll|}
\hline \multicolumn{1}{|l||}{$Q$} & 1 & 2 & 3 \\
\hline-1 & $e^{-}$ & $\mu^{-}$ & $\tau^{-}$ \\
0 & $\nu_{e}$ & $\nu_{\mu}$ & $\nu_{\tau}$ \\
\hline
\end{tabular}

TABLE 1.1: The fundamental fermions of the SM are (a) the quarks and (b) the leptons. Here they are shown in order of increasing generation from left to right (1-3) and are classified into rows of equal electric charge, $Q$, given in units of the proton charge.

field, transforms as the field itself. The result is the introduction of terms in the Lagrangian whereby the spinors couple to vector gauge fields. The coupling strengths are arbitrary and there exist as many gauge fields as there are generators of the symmetry group defining the transformation. Kinetic terms for the freely propagating vector fields also appear. The complete SM of strong and electroweak interactions requires local gauge symmetry for transformations under

$$
S U(3)_{C} \otimes S U(2)_{L} \otimes U(1)_{Y}
$$

The component $\mathrm{SU}(3)_{C}$ represents the coupling of the quarks to the eight gluon vector fields through the colour charge. The remaining $\mathrm{SU}(2)_{L} \times \mathrm{U}(1)_{Y}$ component forms the Glashow-Salam-Weinberg (GSW) model [10-12] of unified electroweak interactions, the structure of which is described below. Masses of the fermions and weak force mediators are generated in the SM through electroweak spontaneous symmetry breaking (SSB) through the Higgs mechanism [13], requiring the addition of the scalar Higgs field.

To date the SM has been incredibly successful in both accurately predicting and accounting for the phenomenology in all experimental observations of the strong, weak and electromagnetic interactions; however, the SM is not a complete theory, it requires a number of arbitrary constants to be input from experimental measurements as it makes no prediction of their magnitude. Furthermore loop corrections relating to calculations of the Higgs mass are quadratically divergent in the $\mathrm{SM}^{1}$ unless its value is fine tuned such that the divergences cancel. These are among the motivations for constructing new physical theories which aim to go beyond the Standard Model (BSM) and provide a more complete picture of fundamental physics. All such theories must reduce to the SM in the correct limits so as to be consistent with the experimental data.

\footnotetext{
${ }^{1}$ The so called hierarchy problem.
} 


\subsubsection{Electroweak Interactions}

The GSW model of electroweak interactions considers left-handed fermion doublets and right-handed fermion singlets, which for a single generation of quarks and leptons are,

$$
\psi_{L}=\left(\begin{array}{c}
u \\
d
\end{array}\right)_{L},\left(\begin{array}{c}
\nu_{e} \\
e^{-}
\end{array}\right)_{L} ; \psi_{R}=e_{R}^{-}, u_{R}, d_{R}
$$

plus corresponding antiparticles. Note that there are implicitly no right-handed neutrinos in the $\mathrm{SM}^{2}$. Interactions are described by the symmetry group,

$$
S U(2)_{L} \otimes U(1)_{Y}
$$

where $S U(2)_{L}$ couples the left-handed fermion doublets to the gauge fields of the weak isospin, $\boldsymbol{I}$, and $U(1)_{Y}$ the fermions to the gauge field of the weak hypercharge, $Y$. Under (1.3) the spinor fields of (1.2) transform as,

$$
\begin{gathered}
\psi_{L} \rightarrow \psi_{L}^{\prime} \equiv e^{i Y \beta(x)} U_{L}(x) \psi_{L} ; \quad U_{L}(x) \equiv e^{i \frac{\sigma_{i}}{2} \alpha_{i}(x)} \quad(i=1,2,3), \\
\psi_{R} \rightarrow \psi_{R}^{\prime} \equiv e^{i Y \beta(x)} \psi_{R} .
\end{gathered}
$$

In (1.4) and (1.5), $Y$ and $\sigma_{i}$ (the Pauli spin matrices) are the generators of the groups $U(1)$ and $S U(2)$ respectively. The four gauge parameters of the transformation are $\beta(x)$ representing a local phase change, and $\alpha_{i}(x)$ representing a local rotation of weak isospin. The required covariant derivatives for the Lagrangian to remain locally invariant are,

$$
\begin{gathered}
D_{\mu}^{(L)} \psi_{L} \equiv\left[\partial_{\mu}+i g \widetilde{W}_{\mu}(x)+i g^{\prime} Y B_{\mu}(x)\right] \psi_{L} ; \quad \widetilde{W}_{\mu}(x)=\frac{\sigma_{i}}{2} W_{\mu}^{i}(x), \\
D_{\mu}^{(R)} \psi_{R} \equiv\left[\partial_{\mu}+i g^{\prime} Y B_{\mu}(x)\right] \psi_{R} .
\end{gathered}
$$

It can be seen that four vector gauge fields are introduced: the $B_{\mu}$ singlet and the $W_{\mu}^{i}$ triplet. Their respective coupling constants are $g^{\prime}$ and $g$. In order for the covariant derivative to transform as the fermion fields the gauge fields must transform as,

$$
\begin{gathered}
B_{\mu}(x) \rightarrow B_{\mu}^{\prime}(x) \equiv B_{\mu}(x)-\frac{1}{g^{\prime}} \partial_{\mu} \beta(x), \\
\widetilde{W}_{\mu}(x) \rightarrow \widetilde{W}_{\mu}^{\prime}(x) \equiv U_{L}(x) \widetilde{W}_{\mu} U_{L}^{\dagger}(x)+\frac{i}{g}\left[\partial_{\mu} U_{L}(x)\right] U_{L}^{\dagger}(x) .
\end{gathered}
$$

\footnotetext{
${ }^{2}$ Experimental evidence of neutrino flavour mixing suggests neutrinos do have finite mass [14]; however, this is not directly relevant to the discussion here and so ignored.
} 
From the above and by adding the correct gauge invariant kinetic terms for the vector fields the electroweak Lagrangian is therefore,

$$
\mathcal{L}_{E W}=i \bar{\psi}_{L} \gamma^{\mu} D_{\mu}^{(L)} \psi_{L}+i \bar{\psi}_{R} \gamma^{\mu} D_{\mu}^{(R)} \psi_{R}-\frac{1}{4} B_{\mu \nu} B^{\mu \nu}-\frac{1}{4} W_{\mu \nu}^{i} W_{i}^{\mu \nu},
$$

where,

$$
\begin{gathered}
B_{\mu \nu} \equiv \partial_{\mu} B_{\nu}-\partial_{\nu} B_{\mu}, \\
W_{\mu \nu}^{i} \equiv \partial_{\mu} W_{\nu}^{i}-\partial_{\nu} W_{\mu}^{i}-g \epsilon^{i j k} W_{\mu}^{j} W_{\nu}^{k} .
\end{gathered}
$$

Here $\epsilon^{i j k}$ is the rank three Levi-Civita tensor. Gauge invariance and the different transformation properties of right and left-handed spinors require the fermions and quanta of the gauge fields in (1.10) to be massless. After SSB the gauge fields mix into physical mass eigenstates,

$$
\begin{gathered}
W_{\mu}^{ \pm}=\frac{1}{\sqrt{2}}\left(W_{\mu}^{1} \mp i W_{\mu}^{2}\right), \\
Z_{\mu}=-B_{\mu} \sin \theta_{W}+W_{\mu}^{3} \cos \theta_{W}, \\
A_{\mu}=B_{\mu} \cos \theta_{W}+W_{\mu}^{3} \sin \theta_{W} .
\end{gathered}
$$

This introduces the weak mixing angle, $\theta_{W}$, which is given by $\tan \theta_{W}=g^{\prime} / g$. Finally the respective couplings of the weak eigenstates of the fermions to the mass eigenstates of the gauge fields, (1.13), (1.14) and (1.15), can be written down as,

$$
\begin{gathered}
-i \frac{g}{\sqrt{2}} \gamma^{\mu} \frac{1}{2}\left(1-\gamma^{5}\right), \\
\frac{-i g}{\cos \theta_{W}} \gamma^{\mu} \frac{1}{2}\left[I_{3}\left(1-\gamma^{5}\right)-2 Q \sin ^{2} \theta_{W}\right], \\
-i e Q \gamma^{\mu} .
\end{gathered}
$$

Here $Q=I_{3}+(Y / 2)$ is the fermion electric charge. It can be seen that the weak charged current, $W^{ \pm}$, couples only to left-handed fermion fields due to the $\left(1-\gamma^{5}\right)$ term in (1.16). The neutral weak current, $Z^{0}$, couples to both left-handed fermions and charged right-handed fermions. Finally the massless photon field, $A_{\mu}$, only couples to the electric charge.

\subsubsection{Charged Current Weak Interactions of Quarks}

SSB through the Higgs mechanism allows the introduction of gauge invariant Yukawa terms in the Lagrangian for each fermion,

$$
\mathcal{L}_{Y}=-Y_{f} \bar{\psi}_{L}^{(f)} \Phi f_{R}+\text { h.c. },
$$


where h.c. stands for hermitian conjugate, $\Phi$ is the scalar Higgs $S U(2)_{L}$ doublet, $Y_{f}$ is the Yukawa coupling for fermion $f$ and $\psi_{L}^{(f)}$ the fermion doublet containing $f_{L}$. The Higgs doublet results in mass terms for each fermion (excluding the neutrino which has no right-handed field in the SM),

$$
-\sqrt{2} \frac{Y_{f} M_{W}}{g}\left(\bar{f}_{L} f_{R}+\bar{f}_{R} f_{L}\right)=\sqrt{2} \frac{Y_{f} M_{W}}{g} \bar{f} f
$$

where $M_{W}$ is the mass of the charged current weak propagator. The fermion mass is directly read from (1.20) as,

$$
m_{f}=\sqrt{2} \frac{Y_{f} M_{W}}{g}
$$

The above holds for a single generation; however, in the three generation SM it is possible to write Yukawa terms which mix quarks of different generations. In this scenario (1.19) for the quarks becomes,

$$
\mathcal{L}_{Y}=\sum_{i, j=0}^{3}\left(\Gamma_{i j}^{u} \bar{\psi}_{L}^{(u) i} \Phi u_{R}^{j}+\Gamma_{i j}^{d} \bar{\psi}_{L}^{(d) i} \Phi d_{R}^{j}+\text { h.c. }\right) ; u=\left(\begin{array}{c}
u \\
c \\
t
\end{array}\right), d=\left(\begin{array}{c}
d \\
s \\
b
\end{array}\right) .
$$

The $\Gamma_{i j}^{q}$ matrix elements mix generations $i, j$ and represent couplings analogous to the $Y_{f}$ of a single generation. It follows that the quark mass matrix has the form,

$$
M_{i j}^{q}=\sqrt{2} \frac{\Gamma_{i j}^{q} M_{W}}{g}
$$

To obtain the physical mass eigenstates of the quarks the mass matrix is diagonalised through the introduction of unitarity matrices, $V_{L(R)}^{q}$, such that,

$$
V_{L}^{q} M^{q} V_{R}^{q \dagger}=\mathcal{M}_{\text {diag }}^{q}
$$

The relation of the quark mass eigenstates to their weak eigenstates is then given by,

$$
u_{L(R)}^{m}=V_{L(R)}^{u} u_{L(R)} ; \quad d_{L(R)}^{m}=V_{L(R)}^{d} d_{L(R)} .
$$

\subsubsection{The Cabibbo-Kobayashi-Maskawa Matrix}

Given (1.25) it is possible to rewrite the electroweak interaction Lagrangian in terms of the quark mass eigenstates. For neutral currents this has no effect, but for charged 
currents,

$$
\begin{aligned}
\mathcal{L}_{C C} & =-\frac{g}{\sqrt{2}} \bar{u}_{L} \gamma^{\mu} W_{\mu}^{+} d_{L}+\text { h.c. } \\
& =-\frac{g}{\sqrt{2}} \bar{u}_{L}^{m} \gamma^{\mu} W_{\mu}^{+}\left(V_{L}^{u} V_{L}^{d \dagger}\right) d_{L}^{m}+\text { h.c. }
\end{aligned}
$$

The up-type quarks thus couple to a linear combination of the down-type quarks and the strength of these couplings are determined by the unitary CKM matrix, $\left(V_{L}^{u} V_{L}^{d \dagger}\right)=$ $V_{C K M}[2,3]$. The CKM matrix can be written as,

$$
V_{C K M}=\left(\begin{array}{ccc}
V_{u d} & V_{u s} & V_{u b} \\
V_{c d} & V_{c s} & V_{c b} \\
V_{t d} & V_{t s} & V_{t b}
\end{array}\right)
$$

From (1.28) it follows that weak charged currents allow transitions between quarks of different generations and that the coupling strengths of these flavour transitions are determined by the weak coupling strength, $g$, scaled by the relevant CKM matrix element $V_{i j}$.

The most general form a unitarity $3 \times 3$ matrix can take is characterised by nine real parameters: three moduli and six phases; however, in the case of the CKM matrix the $U(1)$ global symmetry of the Lagrangian means five of the phases are unobservable as they can be 'absorbed' by redefining the phases of the quark fields. The CKM matrix is therefore completely described by three moduli and one phase. The standard representation as advocated by the Particle Data Group (PDG) [14] is,

$$
V_{C K M}=\left(\begin{array}{ccc}
c_{12} c_{13} & s_{12} c_{13} & s_{13} e^{-i \delta_{13}} \\
-s_{12} c_{23}-c_{12} s_{23} s_{13} e^{i \delta_{13}} & c_{12} c_{23}-s_{12} s_{23} s_{13} e^{-i \delta_{13}} & s_{23} c_{13} \\
s_{12} s_{23}-c_{12} c_{23} s_{13} e^{i \delta_{13}} & -c_{12} s_{23}-s_{12} c_{23} s_{13} e^{i \delta_{13}} & c_{23} c_{13}
\end{array}\right)
$$

where $c_{i j}=\cos \theta_{i j}, s_{i j}=\sin \theta_{i j}$. The angles $\theta_{i j}$ are the three moduli and $\delta_{13}$ represents the phase; $i$ and $j$ are generation indices. A common representation in the study of $B$ meson decays is that of Wolfenstein [15] using the parameters $\lambda, A, \rho, \eta$ whereby,

$$
s_{12} \equiv \lambda ; s_{23} \equiv A \lambda^{2} ; s_{13} e^{i \delta_{13}} \equiv A \lambda^{3}(\rho-i \eta)
$$

Taking $\lambda \approx 0.22$ as an expansion parameter the form of (1.29) in this representation, neglecting terms $\geq \mathcal{O}\left(\lambda^{4}\right)$ is,

$$
V_{C K M}=\left(\begin{array}{ccc}
1-\frac{1}{2} \lambda^{2} & \lambda & A \lambda^{3}(\rho-i \eta) \\
-\lambda & 1-\frac{1}{2} \lambda^{2} & A \lambda^{2} \\
A \lambda^{3}(1-\rho-i \eta) & -A \lambda^{2} & 1
\end{array}\right)
$$


Higher order expansions of the Wolfenstein parameterisation to $\mathcal{O}\left(\lambda^{6}\right)$ exist and under this scheme, to keep concise notation, it is convenient to define the terms [16],

$$
\bar{\rho}=\rho\left(1-\frac{\lambda^{2}}{2}\right) ; \bar{\eta}=\eta\left(1-\frac{\lambda^{2}}{2}\right)
$$

\subsubsection{CP Violation}

Any local quantum field theory constructed in the Lagrangian formalism can be subject to three discrete transformations: parity $(P)$, time reversal $(T)$ and charge conjugation $(C)$. Parity performs the operation $P:(t \rightarrow t ; \mathbf{x} \rightarrow-\mathbf{x})$ and time reversal the operation $T:(t \rightarrow-t ; \mathbf{x} \rightarrow \mathbf{x})$. Charge conjugation substitutes particle for antiparticle, reversing the sign on all internal quantum numbers of the particle while leaving the four-momentum, space-time coordinate and spin unchanged. The combined operation $C P T$ is always a symmetry of the Lorentz invariant Lagrangian [17].

It is possible to rewrite (1.27) as

$$
\mathcal{L}=-\frac{g}{\sqrt{2}}\left[\bar{u}_{i} \gamma^{\mu} W_{\mu}^{+}\left(1-\gamma^{5}\right) V_{i j} d_{j}+\bar{d}_{j} \gamma^{\mu} W_{\mu}^{-}\left(1-\gamma^{5}\right) V_{i j}^{*} u_{i}\right]
$$

Under the discrete transformation $C P$ the terms in this equation transform as

$$
\bar{\psi}_{i} \gamma^{\mu} W_{\mu}\left(1-\gamma^{5}\right) \psi_{j} \stackrel{C P}{\longrightarrow} \bar{\psi}_{j} \gamma^{\mu} W_{\mu}\left(1-\gamma^{5}\right) \psi_{i}
$$

however, $V_{i j}$ and $V_{i j}^{*}$ do not transform. It directly follows that the remaining phase in the CKM matrix can result in the combined $C P$ transformation not being a symmetry of the Lagrangian. It is also required that none of the quarks are mass degenerate and that none of the three CKM moduli are zero or $\pi / 2$ as either would decouple the flavour transition between at least two of the quarks allowing the final CKM phase to be absorbed by a redefinition of quark field phase. $C P$ asymmetry was first observed in 1964 in the neutral kaon system [18] and has since been seen by the BABAR experiment in the neutral $B$ meson system $[19,20]$. Furthermore the $B A B A R$ collaboration have also claimed evidence of $C P$ asymmetry in the charged $B$ meson system [21]. All these results are consistent with the CKM formalism of the SM.

Experimentally there are three types of observable $C P$ violation in the $\mathrm{SM}$ arising from the CKM phase factor [4]. $C P$ violation in decay, known as direct $C P$ violation, occurs when the amplitude, $A$, of a decay is not identical to the conjugate decay amplitude $\bar{A}$. $C P$ violation in mixing, known as indirect $C P$ violation, occurs in the mixing of neutral mesons where the flavour eigenstates are different from the mass eigenstates. This can be observed through the neutral meson weak eigenstate and its anti-particle 
partner decaying to the same $C P$ eigenstate with different amplitudes. The decay $B^{0} \rightarrow$ $J / \psi K_{S}$ and its charge conjugate are an example of such a system. The final type of $C P$ violation occurs from a quantum mechanical interference of the first two. Theoretically $C P$ violation can occur in $B \rightarrow X_{d} \gamma$ transitions [22]; however, the BABAR dataset is insufficient to render the required statistical sensitivity for such a measurement.

\subsubsection{The Unitarity Triangle}

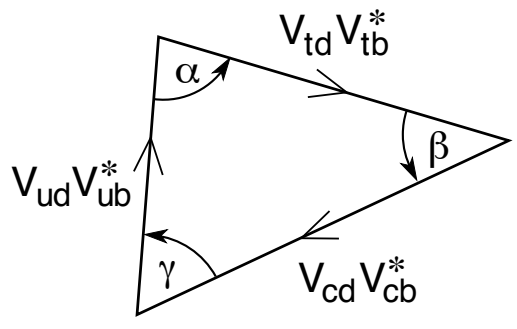

(a)

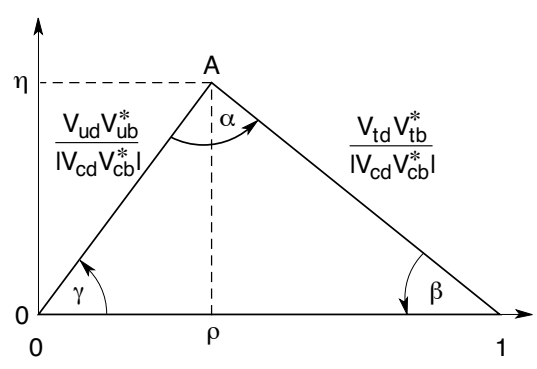

(b)

Figure 1.1: Sketch of the Unitarity Triangle from [4] showing it both as (a) an arbitrary triangle in the complex plane and (b) normalised such that the apex lies at $(\rho, \eta)$.

$C P$ violation in the $\mathrm{SM}$ is constrained by the unitarity of the CKM matrix, $V_{C K M} V_{C K M}^{\dagger}=$ $I$. It follows that testing the constraints of unitarity can test the SM picture of $C P$ violation. There exist six orthogonality relations from unitarity which are each expressed as the vanishing sum of three complex numbers and hence represented as six closed triangles in the complex plane. Of particular interest to $B$ meson decays is the relation,

$$
V_{u d} V_{u b}^{*}+V_{c d} V_{c b}^{*}+V_{t d} V_{t b}^{*}=0
$$

In the complex plane this relation is represented by the so called Unitarity Triangle which is shown in figure 1.1 [4]. It can be seen that it is convenient to normalise the UT by dividing each side by a factor $V_{c d} V_{c b}^{*}$ which results in the apex having coordinate $(\rho, \eta)$ in the Wolfenstein representation. This holds for higher order too so one could easily write $(\bar{\rho}, \bar{\eta})$ as the apex. For very precise experimental measurements of CKM matrix elements and UT triangle geometry the higher order parameterisation is preferred due to its increased analytical accuracy.

Experimentally it is possible to independently measure the angles $\alpha, \gamma$ and $\beta$ as well as the triangle sides. Performing such measurements allows one to determine whether they are consistent with a closed triangle and therefore whether the CKM matrix is unitary and fully accounts for experimental observations of $C P$ violation. The current 


\begin{tabular}{|c|l|}
\hline Param & Value \\
\hline$\lambda$ & $0.2257_{-0.0010}^{+0.0009}$ \\
$A$ & $0.814_{-0.022}^{+0.021}$ \\
$\bar{\rho}$ & $0.135_{-0.016}^{+0.031}$ \\
$\bar{\eta}$ & $0.349_{-0.017}^{+0.015}$ \\
\hline
\end{tabular}

TABLE 1.2: Fitted values of the Wolfenstein parameters from experimental constraints with associated errors [14].

published experimental data are consistent with the UT closing. Measurements for the Wolfenstein parameters are given in table 1.2 [14]. The analysis in this thesis is used to extract a measurement of the ratio of CKM parameters $\left|V_{t d} / V_{t s}\right|$, the details of which are discussed below. It can be seen from (1.31) that in the Wolfenstein parameterisation,

$$
\left|\frac{V_{t d}}{V_{t s}}\right|=\lambda \sqrt{(1-\rho)^{2}+\eta^{2}}
$$

Hence $\left|V_{t d} / V_{t s}\right|$ is directly proportional to the length of the side of the UT between $(\rho, \eta)$ and $(1,0)$ with $\lambda$ as the scaling factor. The form of (1.36) at higher order with $\rho \rightarrow \bar{\rho}$ and $\eta \rightarrow \bar{\eta}$ is discussed below. Typically (1.36) is constrained experimentally from $\left|V_{t d} / V_{t s}\right| \propto\left(\Delta m_{d} / \Delta m_{s}\right)$ where $\Delta m_{d(s)}$ is the mixing frequency of the neutral $B_{d(s)}^{0} \bar{B}_{d(s)}^{0}$ meson system [14]. An independent constraint can be obtained through measuring the rates of electroweak penguin decays and this is the aim of the physics analysis presented in subsequent chapters. Current experimental constraints on $\left|V_{t d} / V_{t s}\right|$ from these different types of analysis are discussed in section 1.3.

\section{$1.2 \quad$ Phenomenology of $B \rightarrow X_{s / d} \gamma$ Decays}

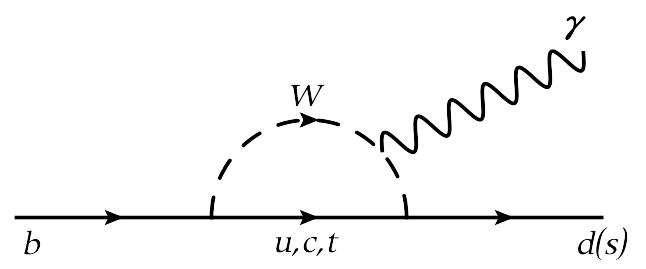

Figure 1.2: Feynman diagram showing the SM loop process dominating the FCNC transition $b \rightarrow s / d \gamma$.

The electroweak formalism of the SM results in there being no flavour changing neutral currents (FCNC) at tree level. The transition $b \rightarrow s / d \gamma$ proceeds at leading order through one-loop penguin processes involving the emission and reabsorption of a $W$ boson combined with an intermediate up-type quark as shown in figure 1.2. The CKM 
and mass dependance of the quark propagator in the loop results in different contributions from each of $u, c$ and $t$, with the more massive $t$ quark dominating. The process $b \rightarrow s / d \gamma$ is thus higher order and suppressed in the SM, with the transition $b \rightarrow d \gamma$ itself suppressed relative to its CKM favoured counterpart, $b \rightarrow s \gamma$. It is possible that contributions from BSM physics can lead to a measurable difference between the experimental rate and the calculated rate from pure SM contributions. Figure 1.3 shows
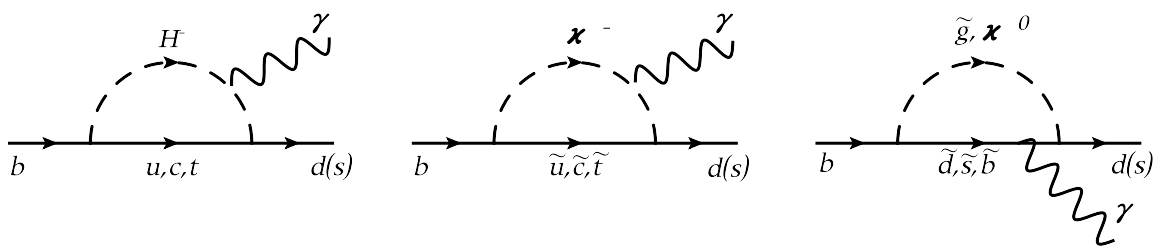

Figure 1.3: Examples of possible BSM contributions to $b \rightarrow s / d \gamma$. The photon can radiate from any charged leg.

possible contributions to the loop from certain supersymmetric models. The presence of such particles can lead to a departure from unitarity of the flavour structure in the quark sector, hence the measurement of these processes perform a stringent test on the validity of the SM at higher order.

The pure electroweak process above is studied through the weak decays of $B$ mesons of the form $B \rightarrow X_{s / d} \gamma$ due to quark confinement. Consequently calculations of the expected SM rates must account for QCD corrections due to strong interactions between the quarks and gluons. Such corrections are complicated by the non-perturbative nature of long range QCD effects which are important when considering hadron decays. The calculation is therefore a non-trivial combination of the electroweak and strong interactions. In order to solve this the formalism of operator product expansion (OPE) is employed.

\subsubsection{Operator Product Expansion}

Consider the amplitude $A$ for a general weak meson $i$ decaying to final state $f$. In the OPE formalism this amplitude is expressed as [23],

$$
A(i \rightarrow f)=\left\langle f\left|\mathcal{H}_{e f f}\right| i\right\rangle=\frac{G_{F}}{\sqrt{2}} \sum_{i} V_{C K M}^{i} C_{i}\left(\mu, M_{W}\right)\left\langle f\left|Q_{i}(\mu)\right| i\right\rangle
$$

where $G_{F}=\left(\sqrt{2} g^{2} / 8 M_{W}^{2}\right)$ is the Fermi constant. The decay amplitude is modelled through an effective theory governed by the Hamiltonian $\mathcal{H}_{\text {eff }}$. This factorises the problem into Wilson coefficient functions, $C_{i}$ and the matrix elements of local operators $Q_{i}$ at a factorisation scale $\mu$. Physically the Wilson coefficients contain the information 
on physics over short distances such as heavy virtual particle exchange (e.g. the $W$ boson or top quark) and short range QCD effects which due to asymptotic freedom can be treated perturbatively. The $Q_{i}$ operators describe the long range interactions, in particular non-perturbative QCD effects which are important in hadronic decays. By definition $A$ is independent of $\mu$ hence the scale dependance of $C_{i}(\mu)$ must cancel that of $Q_{i}(\mu)$. For $B$ meson decays it is usual to take $\mu \sim \mathcal{O}\left(m_{b}\right)$ where $m_{b}$ is the mass of the $b$ quark.

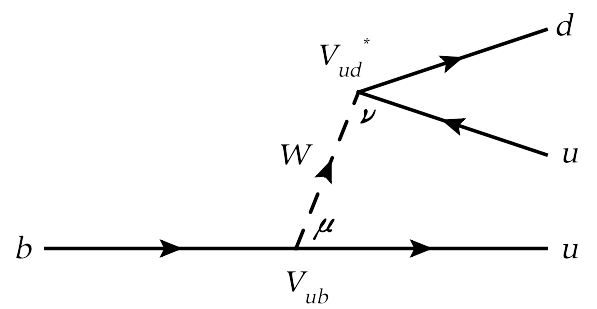

FiguRE 1.4: Feynman diagram showing the tree level weak transition $b \rightarrow u d \bar{u}$.

To illustrate the construction of such an effective theory consider the simple case of the quark level transition $b \rightarrow u d \bar{u}$ shown in figure 1.4 which can contribute to the combinatoric backgrounds of $B \rightarrow X_{s / d} \gamma$ decays if there is a $\pi^{0}$ or $\eta$ meson in the final state. The evaluation of this Feynman diagram yields,

$$
-\frac{g^{2}}{8} V_{u d}^{*} V_{u b}\left[\bar{d} \gamma^{\nu}\left(1-\gamma^{5}\right) u\right]\left[\frac{g_{\nu \mu}}{k^{2}-M_{W}^{2}}\right]\left[\bar{u} \gamma^{\mu}\left(1-\gamma^{5}\right) b\right]
$$

where $k$ is the four-momentum transfer due to the weak boson. Since $k^{2}<m_{b}^{2} \ll M_{W}^{2}$ the following approximation is made,

$$
\frac{g_{\nu \mu}}{k^{2}-M_{W}^{2}} \longrightarrow-\frac{g_{\nu \mu}}{M_{W}^{2}} \equiv-\left(\frac{8 G_{F}}{\sqrt{2} g^{2}}\right) g_{\nu \mu} .
$$

Thus the effective Hamiltonian can be written as,

$$
\mathcal{H}_{e f f}=\frac{G_{F}}{\sqrt{2}} V_{u d}^{*} V_{u b}\left(\bar{d}_{\alpha} u_{\alpha}\right)_{V-A}\left(\bar{u}_{\beta} b_{\beta}\right)_{V-A} \equiv \frac{G_{F}}{\sqrt{2}} V_{u d}^{*} V_{u b} O_{1}
$$

where $\alpha$ and $\beta$ are colour indices and the $V-A$ subscript implies the omitted $\gamma^{\mu}\left(1-\gamma^{5}\right)$ factors. Essentially the $W$ boson has been removed as a degree of freedom from the problem and the transition is described by the local four quark current-current operator $O_{1}$, shown in figure 1.5 .

In this trivial case without QCD corrections the corresponding Wilson coefficient is unity. To account for QCD corrections the effective theory is extended by considering gluon exchanges, examples of which are shown in figure 1.6. The factorisable corrections in 


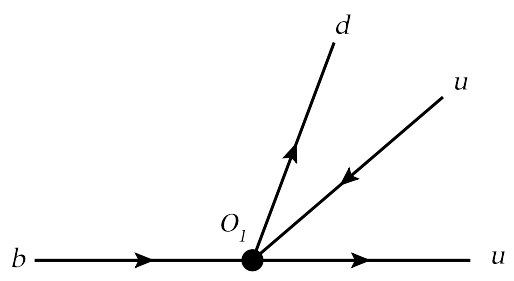

FiguRE 1.5: Four quark current-current operator for the weak transition $b \rightarrow u d \bar{u}$.

figure 1.6(a) result in the Wilson coefficient $C_{1}$ acquiring scale dependance, i.e. $C_{1}(\mu) \neq$ 1. The non-factorisable corrections of figure 1.6(b) require the addition of a second current-current operator from operator mixing,

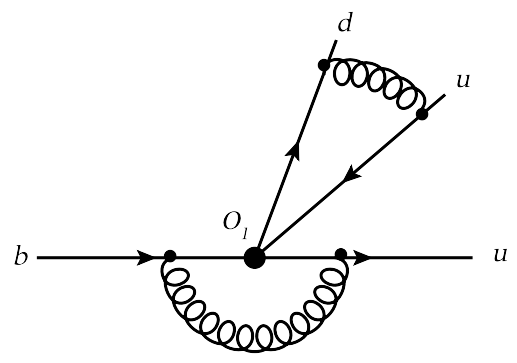

(a)

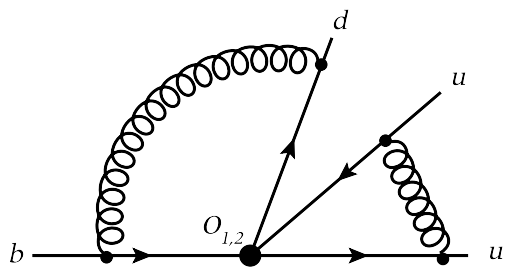

(b)

Figure 1.6: QCD corrections to $b \rightarrow u d \bar{u}$ showing (a) factorisable and (b) nonfactorisable contributions.

$$
O_{2} \equiv\left(\bar{u}_{\alpha} d_{\beta}\right)_{V-A}\left(\bar{u}_{\beta} b_{\alpha}\right)_{V-A}
$$

Hence the effective Hamiltonian becomes,

$$
\mathcal{H}_{e f f}=\frac{G_{F}}{\sqrt{2}} V_{u d}^{*} V_{u b}\left[C_{1}(\mu) O_{1}+C_{2}(\mu) O_{2}\right]
$$

Once the effective Hamiltonian has been constructed, as in the above example, the problem becomes one of evaluating the Wilson coefficients and local operator matrix elements. For the latter there are eight relevant operators in the evaluation of $B \rightarrow X_{s} \gamma$ [24]. Firstly the two current-current operators $Q_{1}$ and $Q_{2}$,

$$
\begin{aligned}
Q_{1} & =\left(\bar{s} T^{a} c\right)_{V-A}\left(\bar{c} T_{a} b\right)_{V-A}, \\
Q_{2} & =(\bar{s} c)_{V-A}(\bar{c} b)_{V-A},
\end{aligned}
$$

where $T_{a}(a=1 \ldots 8)$ are the $S U_{c}(3)$ generators and imply the colour indices of the analogous operator $\mathrm{O}_{2}$ above. Secondly there are four QCD penguin operators which arise from diagrams such as that shown in figure 1.7 and have the form, 


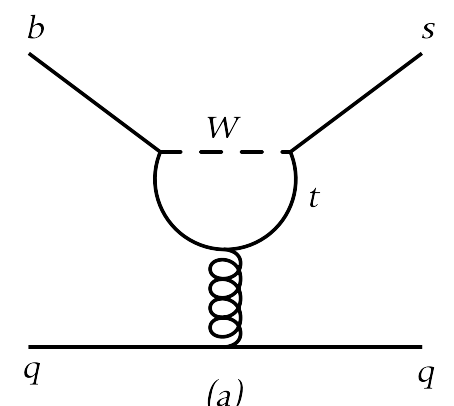

(a)

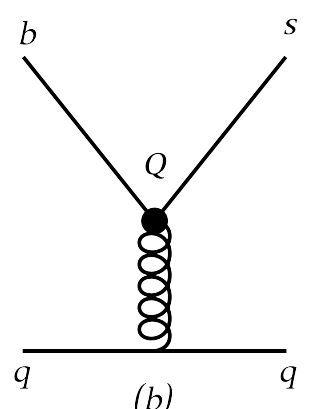

(b)

Figure 1.7: The QCD penguin contributions to $B \rightarrow X_{s} \gamma$, (a) the full SM contribution and (b) the effective operator $Q$ with the heavy degrees of freedom removed. Only the dominant top quark loop is shown.

$$
\begin{aligned}
Q_{3} & =(\bar{s} b)_{V-A} \sum_{q}(\bar{q} q)_{V-A}, \\
Q_{4} & =\left(\bar{s} T^{a} b\right)_{V-A} \sum_{q}\left(\bar{q} T_{a} q\right)_{V-A}, \\
Q_{5} & =(\bar{s} b)_{V-A} \sum_{q}(\bar{q} q)_{V+A}, \\
Q_{6} & =\left(\bar{s} T^{a} b\right)_{V-A} \sum_{q}\left(\bar{q} T_{a} q\right)_{V+A} .
\end{aligned}
$$

These differ from the current-current operators as there are additional right handed contributions from $\gamma^{\mu}\left(1+\gamma^{5}\right)$ terms (denoted $V+A$ ) and there is a sum over all $q \bar{q}$ pairs that the gluon can produce. Finally there are contributions from photon and gluonic magnetic penguin operators shown in figure 1.8 and which have the form,

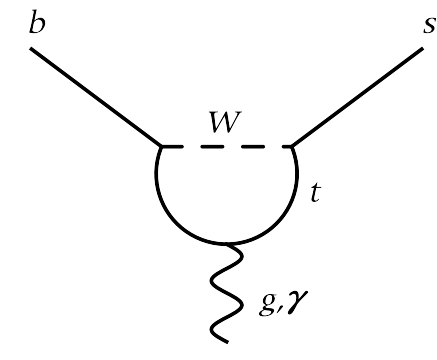

(a)

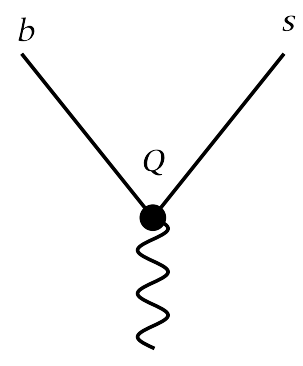

(b)

Figure 1.8: The magnetic penguin contributions to $B \rightarrow X_{s} \gamma$, (a) the full SM contribution and (b) the effective operator $Q$ with the heavy degrees of freedom removed.

The gauge boson leg is a photon for $Q_{7}$ and a gluon for $Q_{8}$.

$$
\begin{aligned}
Q_{7} & =\frac{e}{8 \pi^{2}} m_{b}(\mu) \bar{s} \sigma^{\mu \nu}\left(1+\gamma^{5}\right) b F_{\mu \nu}, \\
Q_{8} & =\frac{g_{s}}{8 \pi^{2}} m_{b}(\mu) \bar{s} \sigma^{\mu \nu} T^{a}\left(1+\gamma^{5}\right) b G_{\mu \nu}^{a},
\end{aligned}
$$


where $e$ and $F_{\mu \nu}\left(g_{s}\right.$ and $\left.G_{\mu \nu}^{a}\right)$ are the electromagnetic (strong) coupling constant and field strength tensor, respectively. For $Q_{7}$ and $Q_{8}, m_{b}(\mu)$ is the running $b$ quark mass. The equivalent $Q_{i}$ operators for $B \rightarrow X_{d} \gamma$ are trivially obtained by substituting the $s$ quark above for a $d$ quark.

\subsubsection{Heavy Quark Expansion}

The $Q_{i}$ operators represent the non-perturbative contributions to the decay $B \rightarrow X_{s / d} \gamma$. In the calculation of exclusive $B$ meson decays the evaluation of $Q_{i}$ can represent a significant and complex challenge; however, in the case of the inclusive decays considered here the problem becomes much simpler. The branching ratio can be calculated using an expansion in inverse powers of $m_{b}[25]$,

$$
\mathcal{B}\left(B \rightarrow X_{q} \gamma\right)=\mathcal{B}(b \rightarrow q \gamma)+\mathcal{O}\left(\frac{1}{m_{b}^{2}}\right)
$$

This is known as heavy quark expansion (HQE). The leading term in (1.51) represents the $b$ quark decay which can be calculated in perturbation theory. In the limit $m_{b} \rightarrow \infty$ the equivalence to the quark level decay is exact and calculations show that the $\mathcal{O}\left(\frac{1}{m_{b}^{2}}\right)$ corrections are $\sim 3 \%$ [22]. Essentially the approximation relies on the fact that the $b$ quark decay is a short distance process whose decay time is much less than the timescale of hadronisation to the final state $X_{q}$. The advantage of the HQE approximation is that the $Q_{i}$ operators can effectively be calculated perturbatively. In particular their scale dependence can be calculated and the cancellation of this dependance with the corresponding $C_{i}(\mu)$ investigated. It follows that under this scheme the long range QCD contributions to the decay $B \rightarrow X_{s / d} \gamma$ are well under control making the decay a laboratory for the short range electroweak effects contained within the Wilson coefficients.

\subsubsection{Wilson Coefficient Evaluation}

The $C_{i}(\mu)$, which contain the short range QCD effects, are always calculable in perturbation theory due to asymptotic freedom of the strong coupling $\alpha_{s}(\mu)$, which at scales $\mu \geq \mathcal{O}(1 \mathrm{GeV})$ can be used as an expansion parameter; however, at the scale $\mathcal{O}\left(m_{b}\right) \ll M\left(M=M_{W}, m_{t}\right)$ large logarithms of the form $\ln (M / \mu)$ multiply $\alpha_{s}$ due to the consequences of evaluating a renormalisable quantum field theory when different scales are present [17]. To maintain the validity of perturbation theory, a renormalisation group analysis is performed allowing summation of the logarithm terms to all orders [25]. The $C_{i}(\mu)$ are therefore evaluated with renormalisation group improved perturbation theory. The leading order (LO) term in such an analysis sums $\left[\alpha_{s} \ln (M / \mu)\right]^{n}$ 
contributions. LO calculations can contain large theoretical uncertainties due to leftover $\mu$ dependance in the amplitude calculation. This is much reduced by additionally summing $\alpha_{s}\left[\alpha_{s} \ln (M / \mu)\right]^{n}$ contributions giving a next to leading order (NLO) calculation. NLO evaluations for all the $C_{i}(\mu)$ relevant to the decay $B \rightarrow X_{s / d} \gamma$ have been made [24].

\subsubsection{Calculation of $\left|V_{t d} / V_{t s}\right|$}

OPE provides a framework to calculate expected SM rates for $B \rightarrow X_{s / d} \gamma$ and the corresponding charge conjugate decay, $\bar{B} \rightarrow \bar{X}_{s / d} \gamma$. The average branching ratio is defined as,

$$
\left\langle\mathcal{B}\left(B \rightarrow X_{s / d} \gamma\right)\right\rangle=\frac{\mathcal{B}\left(B \rightarrow X_{s / d} \gamma\right)+\mathcal{B}\left(\bar{B} \rightarrow \bar{X}_{s / d} \gamma\right)}{2} .
$$

It is possible to relate the ratio,

$$
R(d \gamma / s \gamma) \equiv \frac{\left\langle\mathcal{B}\left(B \rightarrow X_{d} \gamma\right)\right\rangle}{\left\langle\mathcal{B}\left(B \rightarrow X_{s} \gamma\right)\right\rangle}
$$

to the CKM parameter $\left|V_{t d} / V_{t s}\right|$ as shown by Ali et al [22]. A brief summary of their derivation of this relation in terms of the Wolfenstein parameters $\bar{\rho}$ and $\bar{\eta}$ follows.

\subsubsection{Decay Rates in $B \rightarrow X \gamma$}

The effective Hamiltonian for the decay $\bar{B} \rightarrow \bar{X}_{s} \gamma$ leads to the following expression for the decay rate,

$$
\begin{aligned}
\Gamma(b \rightarrow s \gamma) & =\frac{m_{b}^{5} G_{F}^{2} \alpha_{E M}}{32 \pi^{4}}|D(b \rightarrow s \gamma)|^{2} \\
D(b \rightarrow s \gamma) & =\lambda_{t}\left(A_{R e}^{t}+i A_{I m}^{t}\right)+\lambda_{u}\left(A_{R e}^{u}+i A_{I m}^{u}\right),
\end{aligned}
$$

where $\lambda_{q} \equiv V_{q b} V_{q s}^{*}$ are the relevant CKM factors and the real functions $A^{q}$ are constructed from the Wilson coefficients and matrix elements. The branching ratio is expressed in terms of the measured semileptonic branching ratio $\mathcal{B}\left(B \rightarrow X l \nu_{l}\right)$,

$$
\mathcal{B}\left(\bar{B} \rightarrow \bar{X}_{s} \gamma\right)=\frac{\Gamma\left(\bar{B} \rightarrow \bar{X}_{s} \gamma\right)}{\Gamma_{s l}} \mathcal{B}\left(B \rightarrow X l \nu_{l}\right)
$$

where $\Gamma_{s l}$ is the calculated semileptonic rate. This is now written to explicitly show dependancies on the CKM matrix factors,

$$
\mathcal{B}\left(\bar{B} \rightarrow \bar{X}_{s} \gamma\right)=\frac{1}{\left|V_{c b}\right|^{2}}\left[D_{t}\left|\lambda_{t}\right|^{2}+D_{u}\left|\lambda_{u}\right|^{2}+D_{r} \operatorname{Re}\left(\lambda_{t}^{*} \lambda_{u}\right)+D_{i} \operatorname{Im}\left(\lambda_{t}^{*} \lambda_{u}\right)\right]
$$


where the $D$ factors, which depend on various input parameters such as $m_{t}, m_{b}, m_{c}, \mu$ and $\alpha_{s}$, are calculated numerically in table 1 of [22]. The average branching ratio is obtained from dropping the last term of (1.57) due to the corresponding conjugate term having opposite sign. In fact due to the dominance of the leading term it is possible to write,

$$
\left\langle\mathcal{B}\left(B \rightarrow X_{s} \gamma\right)\right\rangle=\frac{\left|\lambda_{t}\right|^{2}}{\left|V_{c b}\right|^{2}} D_{t} .
$$

Following the same procedure for $B \rightarrow X_{d} \gamma$ gives,

$$
\left\langle\mathcal{B}\left(B \rightarrow X_{d} \gamma\right)\right\rangle=\frac{1}{\left|V_{c b}\right|^{2}}\left[D_{t}\left|\xi_{t}\right|^{2}+D_{u}\left|\xi_{u}\right|^{2}+D_{r} \operatorname{Re}\left(\xi_{t}^{*} \xi_{u}\right)\right]
$$

where $\xi_{q} \equiv V_{q b} V_{q d}^{*}$. The $D$ factors of (1.59) are identical to those of (1.57), however, the CKM factors of (1.59) reduce the dominance of the leading term so all terms must be retained.

\subsubsection{2 $\left|V_{t d} / V_{t s}\right|$ from Inclusive Decays}

From (1.58) and (1.59) it follows that,

$$
R(d \gamma / s \gamma)=\frac{\left|\xi_{t}\right|^{2}}{\left|\lambda_{t}\right|^{2}}+\frac{D_{u}}{D_{t}} \frac{\left|\xi_{u}\right|^{2}}{\left|\lambda_{t}\right|^{2}}+\frac{D_{r}}{D_{t}} \frac{\operatorname{Re}\left(\xi_{t}^{*} \xi_{u}\right)}{\left|\lambda_{t}\right|^{2}}
$$

This is rewritten in terms of the Wolfenstein parameters $\bar{\rho}$ and $\bar{\eta}[22]$,

$$
R=\lambda^{2}\left[1+\lambda^{2}(1-2 \bar{\rho})\right]\left[(1-\bar{\rho})^{2}+\bar{\eta}^{2}+\frac{D_{u}}{D_{t}}\left(\bar{\rho}^{2}+\bar{\eta}^{2}\right)+\frac{D_{r}}{D_{t}}\left(\bar{\rho}(1-\bar{\rho})-\bar{\eta}^{2}\right)\right] .
$$

Comparing the leading term of (1.60) to (1.61), the expression for $\left|V_{t d} / V_{t s}\right|$ as a function of the higher order Wolfenstein parameters is found to be,

$$
\frac{\left|\xi_{t}\right|^{2}}{\left|\lambda_{t}\right|^{2}}=X^{2}=\lambda^{2}\left[1+\lambda^{2}(1-2 \bar{\rho})\right]\left[(1-\bar{\rho})^{2}+\bar{\eta}^{2}\right],
$$

where the definition $X=\left|V_{t d} / V_{t s}\right|$ is used to simplify the notation. Remaining terms in (1.61) are also functions of $\bar{\rho}$ and $\bar{\eta}$. If one were to measure $R$ and calculate $X$ while constraining these terms with the current Wolfenstein parameter measurements then the value of $X$ obtained would not be truly independent. This is due to previous measurements of $X$ being used to evaluate the world averages of $\bar{\rho}$ and $\bar{\eta}$. For this analysis the extracted value of $X$ is required to be independent. It is therefore proposed to rewrite (1.61) as a function of $X$ and an orthogonal coordinate which is chosen to be the UT angle $\beta$. It is noticed from (1.62) that in the higher order Wolfenstein expansion $X$ is no longer directly proportional to the relevant side of the UT as was the case for (1.36). Therefore redefinition of the coordinate system requires substitutions of the 
form,

$$
\begin{aligned}
(1-\bar{\rho}) & =\frac{X}{\lambda} \cos \beta+\lambda^{2} f_{\bar{\rho}}(X, \beta), \\
\bar{\eta} & =\frac{X}{\lambda} \sin \beta+\lambda^{2} f_{\bar{\eta}}(X, \beta),
\end{aligned}
$$

where the $f$ functions account for the higher order corrections to (1.36). From (1.62), the relation $\tan \beta=\bar{\eta} /(1-\bar{\rho})$ which is obtained from the UT geometry, assuming $(X / \lambda) \sim 1$ and neglecting terms with multiplying factors $>\mathcal{O}\left(\lambda^{4}\right)$, the $f$ functions are derived to be,

$$
\begin{aligned}
f_{\bar{\rho}}(X, \beta) & =\frac{1}{2} \frac{X}{\lambda} \cos \beta\left[1-2 \frac{X}{\lambda} \cos \beta\right], \\
f_{\bar{\eta}}(X, \beta) & =\frac{1}{2} \frac{X}{\lambda} \sin \beta\left[1-2 \frac{X}{\lambda} \cos \beta\right] .
\end{aligned}
$$

Substituting for $(1-\bar{\rho})$ and $\bar{\eta}$ in (1.61), again assuming $(X / \lambda) \sim 1$ and neglecting terms with multiplying factors $>\mathcal{O}\left(\lambda^{4}\right), R$ is found to be a quadratic in $X$,

$$
R=\kappa_{1} X^{2}+\kappa_{2} X+\kappa_{3}
$$

where,

$$
\begin{aligned}
\kappa_{1} & =1+\frac{D_{u}}{D_{t}}\left(1-2 \lambda^{2} \cos ^{2} \beta\right)-\frac{D_{r}}{D_{t}}\left(\lambda^{2} \cos ^{2} \beta+1\right), \\
\kappa_{2} & =\lambda \cos \beta\left[\frac{D_{u}}{D_{t}}\left(3 \lambda^{2}-2\right)+\frac{D_{r}}{D_{t}}\left(1+\frac{\lambda^{2}}{2}\right)\right], \\
\kappa_{3} & =\lambda^{2} \frac{D_{u}}{D_{t}}\left(1-\lambda^{2}\right) .
\end{aligned}
$$

Numerical calculation of the above $\kappa$ factors uses the world average measurements of $\lambda$ and $\sin 2 \beta$ [14]. The factors $D_{u} / D_{t}$ and $D_{r} / D_{t}$ are taken from table 1 of [22] assuming $\mu=2.5 \mathrm{GeV}$. The current world average of $m_{c} / m_{b}=0.30_{-0.03}^{+0.02}$ does not constrain the numerical variations of the $D$ factors as a function of $m_{c} / m_{b}$ quoted in [22] and hence uncertainty estimates for each $D$ factor are made based on this variation. The complete set of input parameters and their associated uncertainties are show in table 1.3 and give $\kappa_{1}=1.4144, \kappa_{2}=-0.1049$ and $\kappa_{3}=0.0048$. Given the numerical evaluation of the $\kappa$ factors, (1.67) is inverted to obtain $X$ as a function of $R$. The propagation of uncertainties due to the input variables are estimated for the inverted equation. For each input parameter, $Z$, the other parameters are held constant at their central values and $X$ is recalculated using $Z \pm 1 \sigma_{Z}$. Thus two extreme values of $X$ are obtained and the average of the difference of these extremes relative to the central value of $X$ is taken as the error on $X$ due to the variation in $Z$. The parameters $D_{u} / D_{t}$ and $D_{r} / D_{t}$ are 


\begin{tabular}{|c|c|l|}
\hline Param & Value & Error \\
\hline$\lambda$ & 0.2257 & ${ }_{-0.0010}^{+0.0009}$ \\
$\sin 2 \beta$ & 0.681 & \pm 0.025 \\
$\cos \beta$ & 0.931 & ${ }_{-0.007}^{+0.006}$ \\
$\frac{D_{u}}{D_{t}}$ & 0.0995 & ${ }_{-0.0002}^{+0.0005}$ \\
$\frac{D_{r}}{D_{t}}$ & -0.310 & ${ }_{-0.062}^{+0.090}$ \\
\hline
\end{tabular}

TABLE 1.3: Values of input parameters for the evaluation of the numerical $\kappa$ factors required to calculate $X$.

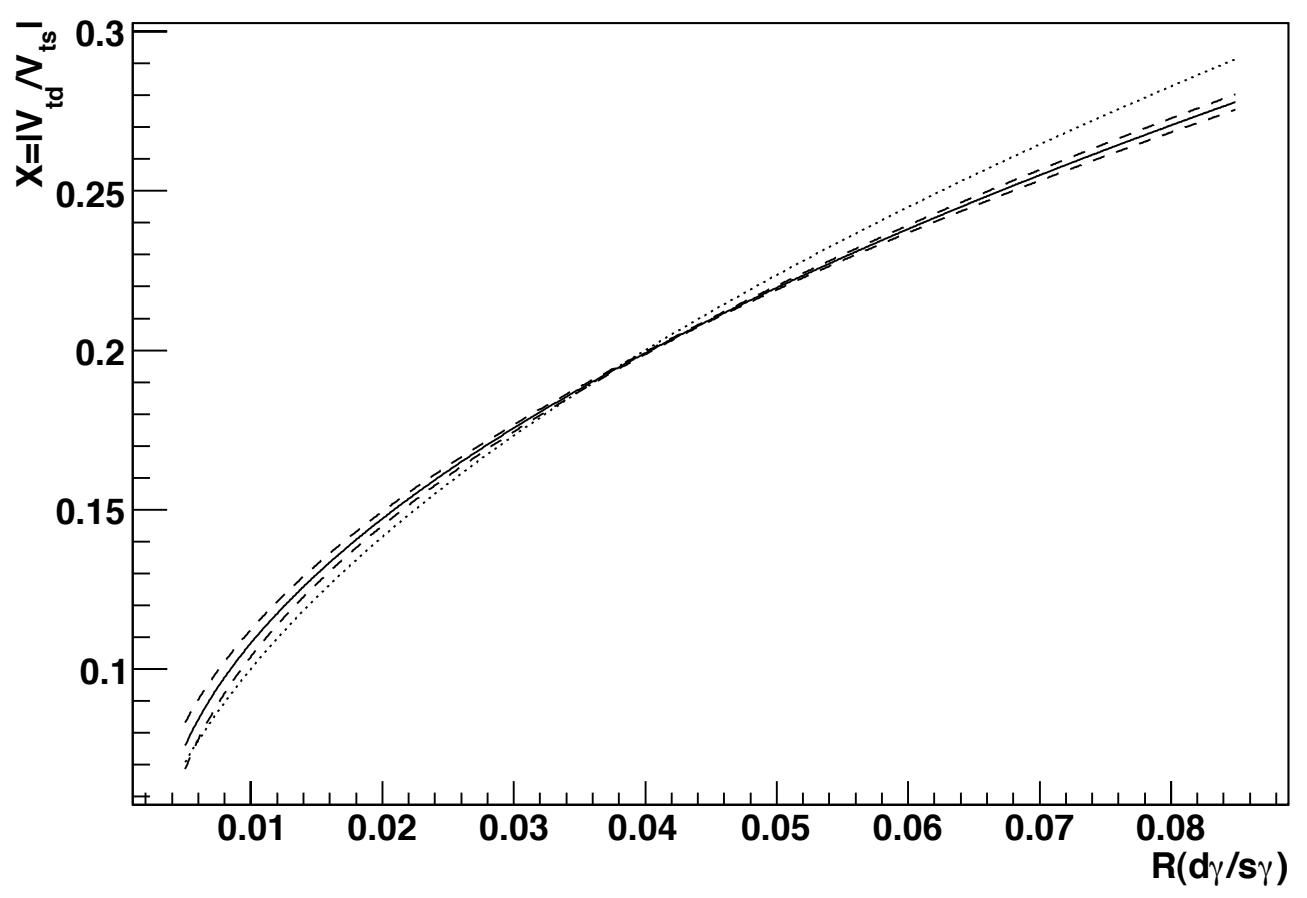

Figure 1.9: Calculated variation of $\left|V_{t d} / V_{t s}\right|$ as a function of $R(d \gamma / s \gamma)$. The solid line is the expected value with the dashed lines showing calculated error. The dotted line shows $\left|V_{t d} / V_{t s}\right|=\sqrt{R}$

varied simultaneously as their dependence on the uncertainty in $m_{c} / m_{b}$ results in their uncertainties being $100 \%$ correlated. The three individual uncertainties are added in quadrature to obtain an overall error on $X$. The numerical evaluation of (1.67) with corresponding uncertainties is shown in figure 1.9. For the range of $R$ plotted, the relative uncertainty on $X$ is seen to vary with a maximum of $\mathcal{O}(9 \%)$ at lower $R$ and a minimum of $\mathcal{O}(0.2 \%)$ towards the centre of the distribution. Also plotted is the leading term of (1.60), $X=\sqrt{R}$ to illustrate the effect of higher order corrections. The dominant uncertainties in figure 1.9 are due to uncertainties in the numerical evaluation of the $D$ factors. It follows that when corrections from the $D$ factors become less important, 
i.e. when the calculated distribution coincides with the leading order distribution at $R \approx 0.04$, the theoretical uncertainty is significantly reduced.

\subsubsection{3 $\left|V_{t d} / V_{t s}\right|$ from Exclusive Resonant $B \rightarrow X_{s / d} \gamma$ Decays}

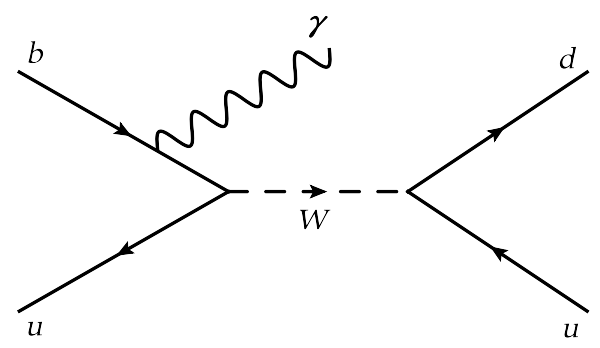

FIGURE 1.10: Feynman diagram showing weak annihilation contributions in the SM to the decay $B^{-} \rightarrow \rho^{-} \gamma$.

Previous measurements have used radiative penguin decays to extract $X$ by taking the ratio of exclusive resonant decays and applying the calculated theoretical relation [26],

$$
\frac{\Gamma(B \rightarrow \rho \gamma)}{\Gamma\left(B \rightarrow K^{*} \gamma\right)}=S_{\rho}\left|\frac{V_{t d}}{V_{t s}}\right|^{2}\left(\frac{1-m_{\rho}^{2} / M_{B}^{2}}{1-m_{K^{*}}^{2} / M_{B}^{2}}\right)^{3} \zeta^{2}[1+\Delta R]
$$

Here $S_{\rho}$ is an isospin factor dependent on the charge of the $\rho$ meson, $m_{\rho\left(K^{*}\right)}$ is the mass of the $\rho\left(K^{*}\right)$ meson, $M_{B}$ the mass of the $B$ meson, $\zeta$ a form factor ratio, and $\Delta R$ a correction factor for weak annihilation contributions which are shown in figure 1.10. To date such measurements show no discrepancy between the extracted values of $X$ compared to those measured from $B$ meson mixing. However, they are not as competitive as the latter due to limited statistics in the measurement of $B \rightarrow \rho \gamma$ and due to a relatively large theoretical error in $\zeta$ of $\sim 8 \%$. The independence of different operators contributing to the relevant rate calculations for these respective processes means BSM physics could be observable in only one of these measurements and hence the increased uncertainty in radiative penguin measurements does not diminish the importance of their measurement. The significantly reduced theoretical error from the ratio of inclusive decays in extracting $X$ means that the value obtained from the analysis presented here will be competitive with the measurement from exclusive decays.

\subsubsection{Kagan-Neubert Model of Photon Energy Spectrum}

The experimental interpretation of inclusive $B \rightarrow X_{s / d} \gamma$ decays must take account of the spectrum of photon energy ${ }^{3}, E_{\gamma}$. A number of theoretical schemes can be used to

\footnotetext{
${ }^{3}$ Defined in the $B$ meson rest frame.
} 
estimate this spectrum for $B \rightarrow X_{s} \gamma$ decays [27-29]. This analysis uses the model of Kagan and Neubert (KN) which is comprehensively described in [27] and summarised here. Although older than other schemes it is chosen as it is readily implemented in the $B A B A R$ simulation framework and has been shown to be in reasonable agreement with previous BABAR experimental investigations [30].

\subsubsection{Photon Energy Spectrum}
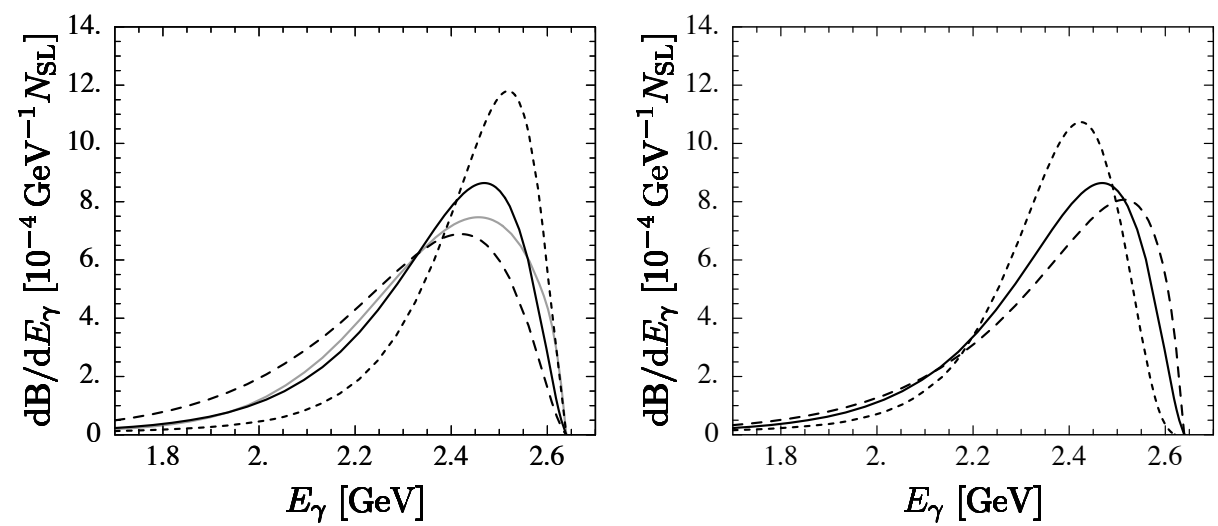

Figure 1.11: Plots taken from Figure 3 of [27] showing normalised photon spectra for $B \rightarrow X_{s} \gamma$ decays using various numerical parameterisations of the shape function $F(k)$ defined in (1.74). The left plot shows variations in $m_{b}$ with $4.65 \mathrm{GeV} / c^{2}$ the long-dashed line, $4.8 \mathrm{GeV} / c^{2}$ the solid line and $4.95 \mathrm{GeV} / c^{2}$ the short-dashed line. The grey curve represents a Gaussian parameterisation of $F(k)$ and is not relevant to the discussion here. The right plot shows variations in $\mu_{\pi}^{2}$ with $0.15 \mathrm{GeV}^{2}$ the short-dashed line, $0.30 \mathrm{GeV}^{2}$ is the solid line and $0.45 \mathrm{GeV}^{2}$ the long-dashed line.

The KN model calculates $\mathcal{B}\left(B \rightarrow X_{s} \gamma\right)$ at NLO and then considers the phenomenological contribution of so called 'Fermi motion' of the $b$ quark to HQE. This motion is modelled as a shape function, $F(k)$, which governs the light-cone momentum distribution, $k$, of the $b$ quark within the $B$ meson which has mass $m_{B}$. The photon spectrum is then calculated as a function of the parameter $y=2 E_{\gamma} / m_{B}$ from a convolution of the parton model spectrum $P_{p}\left(y_{p}\right)$ and the shape function,

$$
P(y) \mathrm{d} y=\int \mathrm{d} k F(k)\left[P_{p}\left(y_{p}\right) \mathrm{d} y_{p}\right]_{y_{p}=y_{p}(k)} .
$$

Here $y_{p}(k)=2 E_{\gamma} / m_{B}^{*}$ with effective mass $m_{B}^{*}=m_{B}+k$ ensuring the Fermi motion model gives the true kinematic limit $E_{\gamma}^{\max }=m_{B} / 2$ rather than $E_{\gamma}^{\max }=m_{b} / 2$ expected from the parton model. 
The shape function is found to be theoretically constrained by the moments,

$$
A_{n}=\int \mathrm{d} k k^{n} F(k)
$$

with $A_{0}=1, A_{1}=0$ and $A_{2}=\frac{1}{3} \mu_{\pi}^{2}$. The $\mathrm{KN}$ model choses a parameterisation of $F(k)$ consistent with these constraints,

$$
F(k)=N(1-x)^{a} e^{(1+a) x} ; \quad x=\frac{k}{\Lambda},
$$

where $N$ is a normalisation factor fixed by $A_{0}, \Lambda=m_{B}-m_{b}$ and $A_{2}=\Lambda^{2} /(1+a)$. It follows that $F(k)$ is parameterised by $\left(m_{b}, \mu_{\pi}^{2}\right)$.

Figure 1.11 shows various normalised evaluations of the photon spectra for $B \rightarrow X_{s} \gamma$ decays. The effects of varying each shape function parameter, $\left(m_{b}, \mu_{\pi}^{2}\right)$, while keeping the other constant are shown. This analysis models the photon spectrum with $\left(4.65 \mathrm{GeV} / c^{2}, 0.52 \mathrm{GeV}^{2}\right)$ based on fits to $b \rightarrow s \gamma$ and $b \rightarrow c l \nu$ data [31]. For systematic studies these parameters are varied in a correlated way ${ }^{4}$, according to their measured uncertainties, to give alternative photon spectra with $\left(4.60 \mathrm{GeV} / c^{2}, 0.60 \mathrm{GeV}^{2}\right)$ and $\left(4.70 \mathrm{GeV} / c^{2}, 0.45 \mathrm{GeV}^{2}\right)$.

\subsubsection{Hadronic Mass Spectrum}

When considering different frames of reference (such as the $B$ meson rest frame, the $\Upsilon(4 S)$ rest frame and the laboratory rest frame) it is useful to parameterise the photon energy spectrum as a hadronic mass spectrum of the $X_{s / d}$ system, $m_{X}$. This is because the Lorentz invariance of $m_{X}$ results in identical spectra in all rest frames. In the $B$ meson rest frame,

$$
m_{X}^{2}=m_{B}^{2}-2 m_{B} E_{\gamma}
$$

The left hand plot of figure 1.12 shows the mass spectrum corresponding to each of the photon spectra in the left hand plot of figure 1.11. The treatment of mass spectra in [27] recognises that their distribution assumes quark-hadron duality. However, the true mass spectra will have contributions with $X_{s / d}=V$, where $V$ is a vector resonance. Of these only the lowest lying resonances, $K^{*}(892), \rho$ and $\omega$ are narrow enough to significantly distort the spectrum shape and potentially invalidate the assumption of quark hadron duality. For this reason the hadronic mass is split into two regions, a low mass resonant region and a high mass continuum of states. This is illustrated in the right hand plot of

\footnotetext{
${ }^{4}$ Although the parameters are essentially independent there is a naive expectation that $\mu_{\pi}^{2}$ increases as $m_{b}$ decreases. Previous BABAR analyses therefore make correlated systematic variations to reflect this and this analysis maintains that convention.
} 

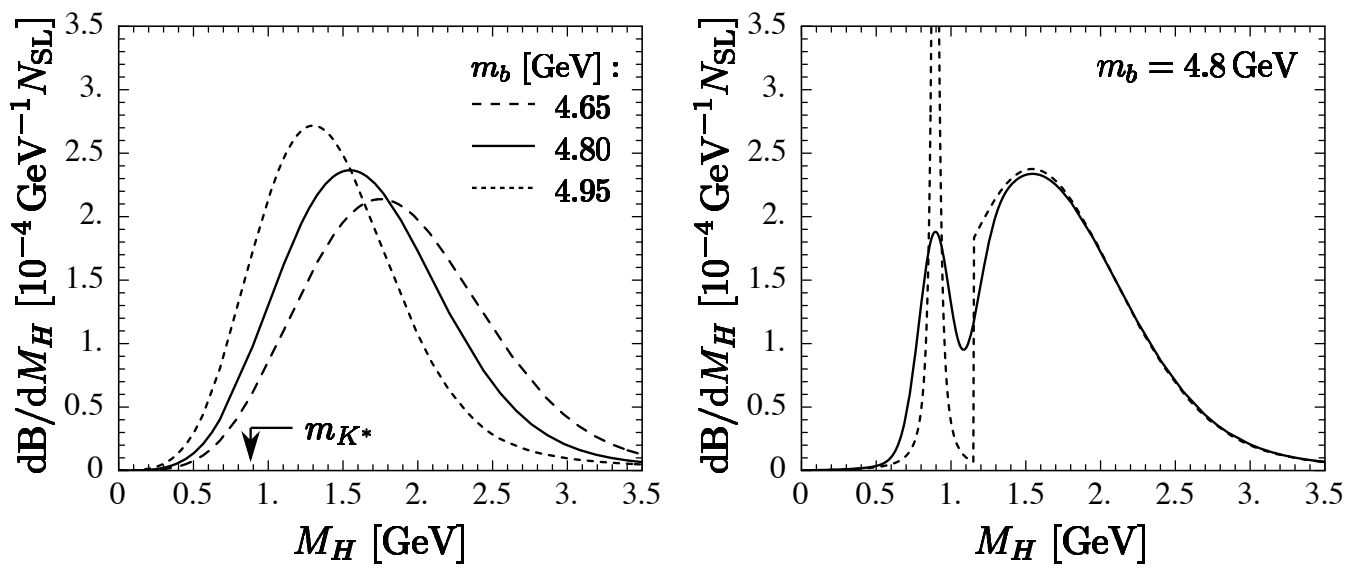

Figure 1.12: Plots taken from Figure 8 of [27] showing normalised hadronic mass spectra for $B \rightarrow X_{s} \gamma$ decays. The authors of this paper use the symbol $M_{H}$ in place of $m_{X}$ which is used in the text of this thesis. The left plot shows variations in $m_{b}$ with $4.65 \mathrm{GeV} / c^{2}$ the long-dashed line, $4.8 \mathrm{GeV} / c^{2}$ the solid line and $4.95 \mathrm{GeV} / c^{2}$ the short-dashed line. The right plot shows a combined mass spectrum separating the low mass resonant region from the high mass region where there is a continuum of states.

The relevance of the dashed and solid lines are described in the text.

figure 1.12 for $B \rightarrow X_{s} \gamma$ decays. The $K^{* 5}$ peak is modelled as a relativistic Breit-Wigner (BW) distribution ${ }^{6}$ and the continuum of states is introduced at a threshold, $m_{X}^{T}$, as illustrated by the dashed line. The solid line shows this spectrum model convoluted with a Gaussian distribution of width $\sigma=100 \mathrm{MeV} / c^{2}$, to emulate potential experimental resolution effects of hadronic mass reconstruction. The model used for the analysis presented here emulates the dashed line with $m_{X}^{T}=1.0 \mathrm{GeV} / c^{2}$ and assumes that to a good approximation such a mass spectrum does not invalidate the assumption of quarkhadron duality [27].

\section{$1.3 \quad$ Experimental Measurements of $\left|V_{t d} / V_{t s}\right|$}

The following section briefly summarises the existing experimental measurements of $\left|V_{t d} / V_{t s}\right|$ from both $B$ mixing and radiative penguin decays.

\subsubsection{Measurement from $B$ Mixing}

Figure 1.13 shows the dominant Feynman diagram contributing to neutral $B$ meson mixing. As with radiative penguin transitions the more massive $t$ quark dominates the

\footnotetext{
${ }^{5}$ Unless otherwise stated $K^{*}$ refers to the $K^{*}(892)$ resonance.

${ }^{6}$ The BW distribution has the form $|\mathcal{A}(s)|^{2}=m_{0}^{2} \Gamma^{2} /\left[\left(s-m_{0}^{2}\right)^{2}+\left(m_{0} \Gamma^{2}\right)\right]$ where $s$ is the square of the centre of mass energy, $m_{0}$ is the resonance pole and $\Gamma$ the resonance width.
} 


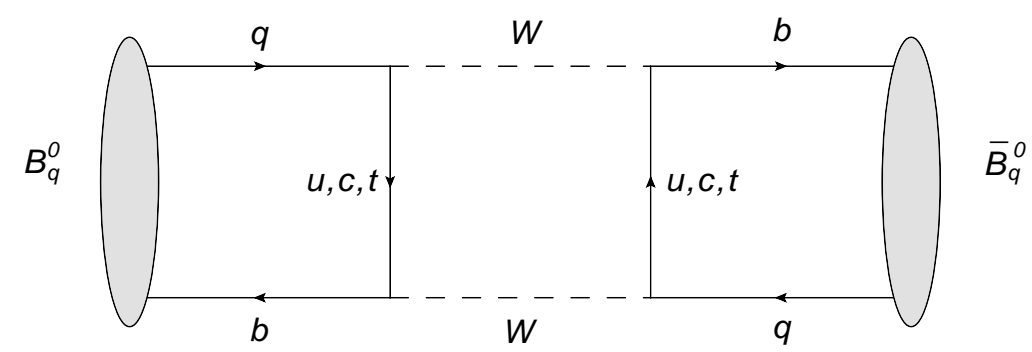

Figure 1.13: Dominant Feynman diagram contributing to neutral $B$ meson mixing. The $B$ meson spectator quark $q=d, s$.

virtual fermion exchange. In $B_{d}^{0} \rightarrow \bar{B}_{d}^{0}$ oscillation (where $B_{d}^{0}=|\bar{b} d\rangle$ ) the charged weak current has contributing factors from $V_{t d}$ and $V_{t d}^{*}$. In the transition $B_{s}^{0} \rightarrow \bar{B}_{s}^{0}$ (where $\left.B_{s}^{0}=|\bar{b} s\rangle\right)$ these factors are substituted for $V_{t s}$ and $V_{t s}^{*}$ respectively. It can be shown that [14],

$$
\left|\frac{V_{t d}}{V_{t s}}\right|^{2}=\xi_{Q C D}^{2} \frac{\Delta m_{d}}{\Delta m_{s}} \frac{M_{B_{s}^{0}}}{M_{B_{d}^{0}}},
$$

where $\xi_{Q C D}^{2}$ is a theoretical factor calculated using Lattice QCD techniques [14] and $\Delta m_{q}$ and $M_{B_{q}^{0}}$ are the respective mixing frequency and mass of the $B_{q}^{0}$ system. The CDF collaboration measure $\Delta m_{s}=17.77 \pm 0.12 \mathrm{ps}^{-1}$ and combining this with the world average $\Delta m_{d}=0.507 \pm 0.005 \mathrm{ps}^{-1}$ they calculate $\left|V_{t d} / V_{t s}\right|=0.209 \pm 0.001 \pm 0.006$ [32] where the first error is experimental and the second error theoretical.

\subsubsection{Measurements from Radiative Penguin Decays}

The BABAR and BELLE collaborations have measured the exclusive radiative penguin transitions $B \rightarrow \rho \gamma[33,34]$. As discussed above such measurements can be combined with world average $B \rightarrow K^{*} \gamma$ measurements [14] to extract $\left|V_{t d} / V_{t s}\right|$. The BABAR measurements give $\left|V_{t d} / V_{t s}\right|=0.233 \pm 0.025 \pm 0.022[33]$ and BELLE measure $\left|V_{t d} / V_{t s}\right|=$ $0.195 \pm 0.020 \pm 0.015$ [34]. In both cases the first error is experimental and second error theoretical. The BELLE result has an improved theoretical error as their result takes advantage of a more recent theoretical calculation. The previous version of this analysis measures $\left|V_{t d} / V_{t s}\right|=0.177 \pm 0.043 \pm 0.001[1]$.

\subsubsection{Summary of $\left|V_{t d} / V_{t s}\right|$ Measurements}

To date the measurement from neutral $B$ meson mixing is clearly more accurate, with almost negligible experimental error and relatively small theoretical error from Lattice QCD calculations. Although less competitive the radiative penguin measurements are 
currently consistent with the $B$ mixing result; exclusive decay modes have similar experimental and theoretical errors whereas the previous version of this analysis is limited by the experimental error. The aim of the analysis presented in this thesis is to significantly reduce the experimental error of the previous measurement. 


\section{Chapter 2}

\section{The BABAR Experiment}

The BABAR experiment collected $e^{+} e^{-}$annihilation data from the interaction region of the energy-asymmetric PEP-II storage rings at the Stanford Linear Accelerator Center (now the SLAC National Accelerator Laboratory) in California, USA. Running from May 1999 until April 2008 it was designed to experimentally probe the theoretical description of heavy flavour physics in the SM. The primary physics goal from the analysis of BABAR data, as outlined in the Technical Design Report [35], is the study of $C P$ violating asymmetries in the decays of $B^{0}$ mesons to $C P$ eigenstates. For example the measurement of $C P$ asymmetries between the decay $B^{0} \rightarrow J / \psi K_{S}^{0}$ and its charge conjugate constrains the SM parameter $\sin 2 \beta$, where $\beta$ is an angle of the Unitarity Triangle discussed in the previous chapter. Additional physics goals include measurements of $B$ meson decays sensitive to different elements of the CKM matrix, with the ultimate aim of imposing redundant constraints on its parameters. The full dataset of just under $0.5 \mathrm{ab}^{-1}$ also allows rare $B$ decays with branching fractions of order $10^{-6}$ to be studied. Finally the clean environment and high luminosities of the BABAR experiment permits precision measurement of many $\tau$ and charm decays. Overall $B A B A R$ has a very rich physics program, more details of which can be found in the BABAR physics book [4] and from browsing any of the physics publications from the BABAR collaboration, which now number in excess of 350 papers.

This chapter provides a concise description of the PEP-II collider and BABAR detector. Emphasis is placed on $B$ meson production and on the tracking, particle identification and calorimetry of both primary and secondary daughter particles from $B$ meson decays as these aspects are the most relevant to the analysis described in this thesis. Technical specifications and performance requirements ${ }^{1}$ of the relevant detector subsystems in each of these categories are discussed.

\footnotetext{
${ }^{1}$ Despite the BABAR experiment no longer being operational, in this thesis all design specifications and performance requirements are described in the present tense.
} 


\subsection{PEP-II Asymmetric $e^{+} e^{-}$Collider}

The PEP-II asymmetric-energy two-storage-ring accelerator [36] collided bunches of electrons and positrons at a centre of mass $(\mathrm{CM})$ energy of $10.58 \mathrm{GeV}$. This energy corresponds to the mass of the $\Upsilon(4 S)$ [14], an S-wave $b \bar{b}$ bound resonant state which is the first of the excited $\Upsilon(n S)$ states above the $B \bar{B}$ threshold. The $\Upsilon(4 S)$ accounts for almost $25 \%$ of the hadronic $e^{+} e^{-}$cross section at this energy and decays almost exclusively to $B \bar{B}$ pairs $^{2}$ [4]. The PEP-II design luminosity of $3 \times 10^{33} \mathrm{~cm}^{-2} \mathrm{~s}^{-1}$ corresponds to a $3 \mathrm{~Hz}$ production rate of $B$ meson pairs, making it a suitable laboratory for the study of their decays. Due to the low Q-value of the decay $\Upsilon(4 S) \rightarrow B \bar{B}, B$ mesons in the CM frame are produced almost at rest. In order to accurately measure the flight length difference, and hence decay time differnece of a $B \bar{B}$ pair, their decay vertices must be well separated in the laboratory frame. For this reason $9.0 \mathrm{GeV}$ electrons are collided with $3.1 \mathrm{GeV}$ positrons head-on resulting in the $\Upsilon(4 S)$ obtaining a Lorentz boost of $\beta \gamma=0.56$ in the laboratory frame.

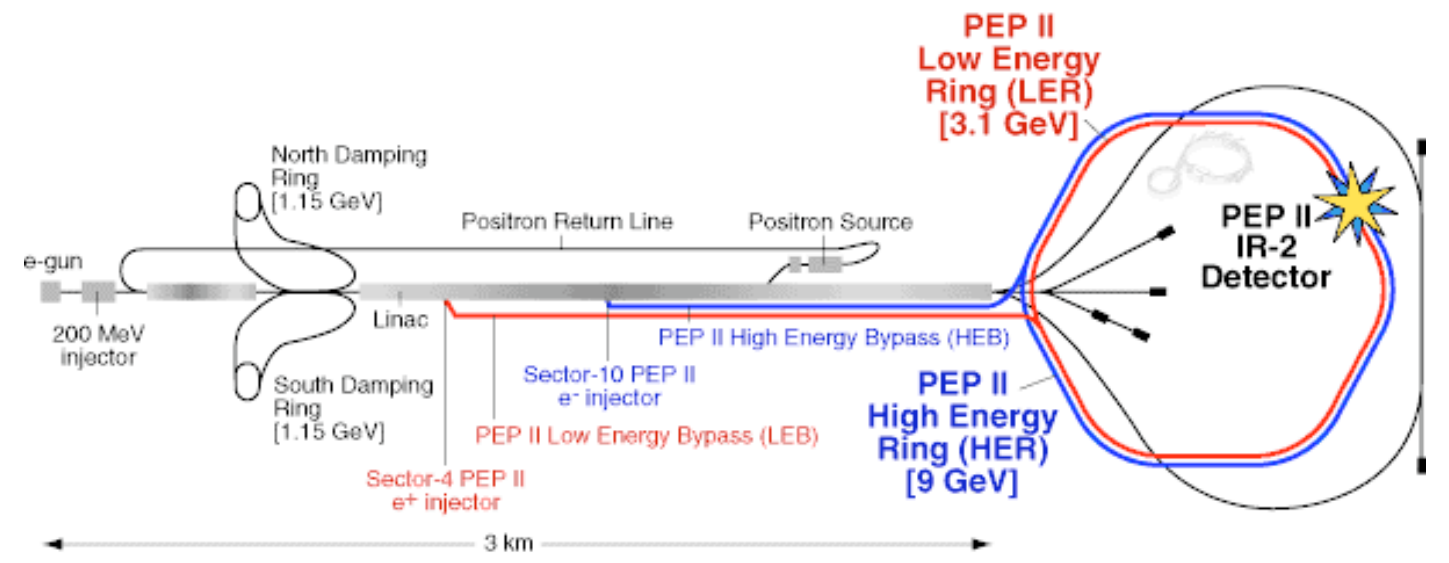

FIguRE 2.1: Schematic diagram of the PEP-II storage rings and linear accelerator.

Figure 2.1 shows a schematic representation of the PEP-II storage rings and the linear accelerator (linac) from which electrons and positrons were injected. Electrons bunches, produced from an electron gun at the end of the linac, are accelerated to around $1 \mathrm{GeV}$ and fed into a damping ring where they are condensed to higher densities required for collision. Positrons are created from colliding further electrons accelerated to around $30 \mathrm{GeV}$ with a tungsten-rhenium target and then collected in bunches. These are also accelerated to around $1 \mathrm{GeV}$ and fed into a separate damping ring. From the damping rings both electrons and positrons are accelerated along the linac, using $\mathrm{RF}$ cavities

${ }^{2} B \bar{B}$ refers to both $B^{0} \bar{B}^{0}$ and $B^{+} B^{-}$pairs. 
powered by klystrons, to energies of $9.0 \mathrm{GeV}$ and $3.1 \mathrm{GeV}$ respectively. They are then injected into the PEP-II storage rings: the electron beam, travelling clockwise, into the so-called high energy ring (HER) and the positron beam, travelling anti-clockwise, into the corresponding low energy ring (LER). Both beams are brought into collision at the interaction region (IR) surrounded by the BABAR detector. The beams are focused with sets of quadrupole magnets before colliding in the IR and then steered away from each other using dipole magnets. Instantaneous luminosity measurements are made by detecting radiative Bhabha photons emitted by incoming positrons [37]. PEP-II achieved a record instantaneous luminosity of $12.1 \times 10^{33} \mathrm{~cm}^{-2} \mathrm{~s}^{-1}$, which is more than four times the design.

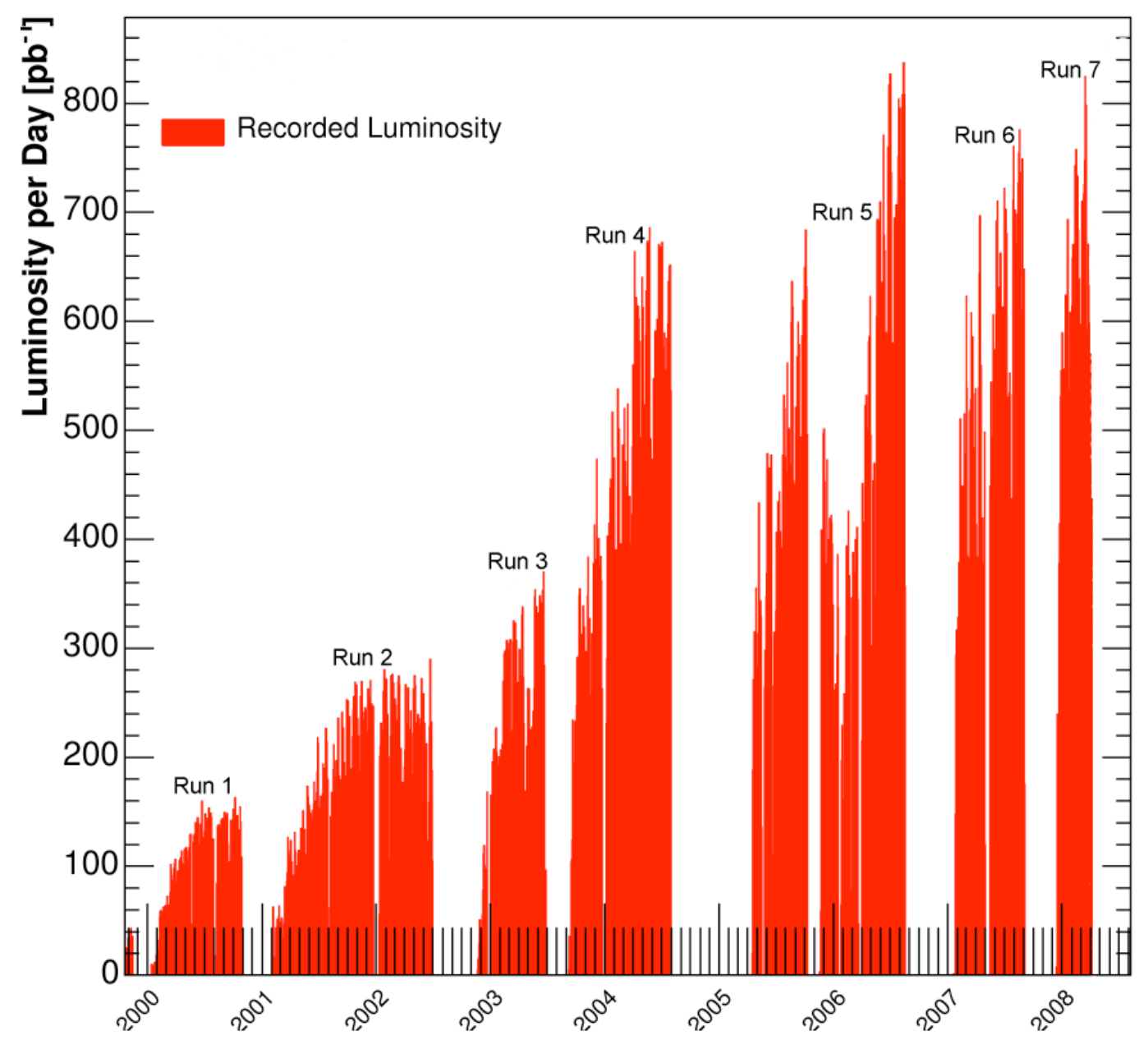

Figure 2.2: Plot showing the integrated luminosity per day of the PEP-II collider for the duration of its running period.

The total integrated luminosity of data collected by BABAR at the IR of the PEP-II collider is $531.4 \mathrm{fb}^{-1}$, a detailed breakdown of which is shown in figure 2.2. Of these data $432.9 \mathrm{fb}^{-1}$ were recorded at the $\Upsilon(4 S) \mathrm{CM}$ energy, with an additional $45.3 \mathrm{fb}^{-1}$ 
collected at an energy just below the resonance to allow simulation independent studies of hadronic backgrounds from $e^{+} e^{-} \rightarrow q \bar{q}(q=u, d, s, c)$ processes, which in this thesis are referred to as 'continuum' events. Data collection periods are divided into distinct intervals each known as a 'Run'. There are a total of seven data collection Runs which are indicated on figure 2.2. The time between running periods was used to perform both detector and accelerator maintenance and upgrades. Data from Runs 1-6 were collected at or just below the $\Upsilon(4 S)$ resonance and so form the full dataset used for the analysis described in the subsequent chapters. Run 7 collected the remaining $53.2 \mathrm{fb}^{-1}$ of data at or just below both the $\Upsilon(2 S)$ and $\Upsilon(3 S)$ resonances and was ended with an 'energy scan' above the $\Upsilon(4 S)$ resonance, it therefore does not form part of the analysis dataset used in this thesis.

\subsection{The BABAR Detector}

The BABAR detector is comprehensively described in [38], a summary of which is given here. It is typical of most modern detectors in high energy physics in that it consists of a series of sub-detectors arranged in a nested cylindrical onion-like structure around the IR. Each sub-detector is dedicated to measuring a particular characteristic of the long-lived daughter particles produced from initial interactions. Here long-lived refers to either stable particles (i.e. electrons, protons and photons) or those whose average lifetime is sufficiently long (e.g. muons, positrons ${ }^{3}$, charged pions and charged kaons) that there is a high probability they interact with one or more of the BABAR sub-detectors. Generally each sub-detector design is optimised to measure the properties of a subset of long-lived particles. Ultimately these measured properties are used to infer the character of primary particle decays and hence extract the physics of interest from an event.

Figure 2.3 shows longitudinal and transverse cross-sections of the BABAR detector. Each of the sub-detectors are labelled and in order of increasing distance from the IR these consist of: a silicon vertex tracker (SVT) and drift chamber (DCH) for charged particle detection; a detector of internally reflected Cherenkov radiation (DIRC) used in charged particle identification; an electromagnetic calorimeter (EMC) for the detection of photons and identification of electrons; and an instrumented flux return (IFR) for muon detection. A superconducting solenoid, surrounding all sub-detectors with the exception of the IFR, produces a $1.5 \mathrm{~T}$ uniform magnetic field along the principal axis of the drift chamber for the measurement of charged track momenta. The collision axis is offset from this axis by about $20 \mathrm{mrad}$ to minimise the effect of the solenoid field on the colliding beams. In figure 2.3 it can be seen that the BABAR detector itself has an

\footnotetext{
${ }^{3}$ Positrons are of course stable particles, however, in the presense of the matter of the BABAR detector there is a high probability they will annihilate.
} 


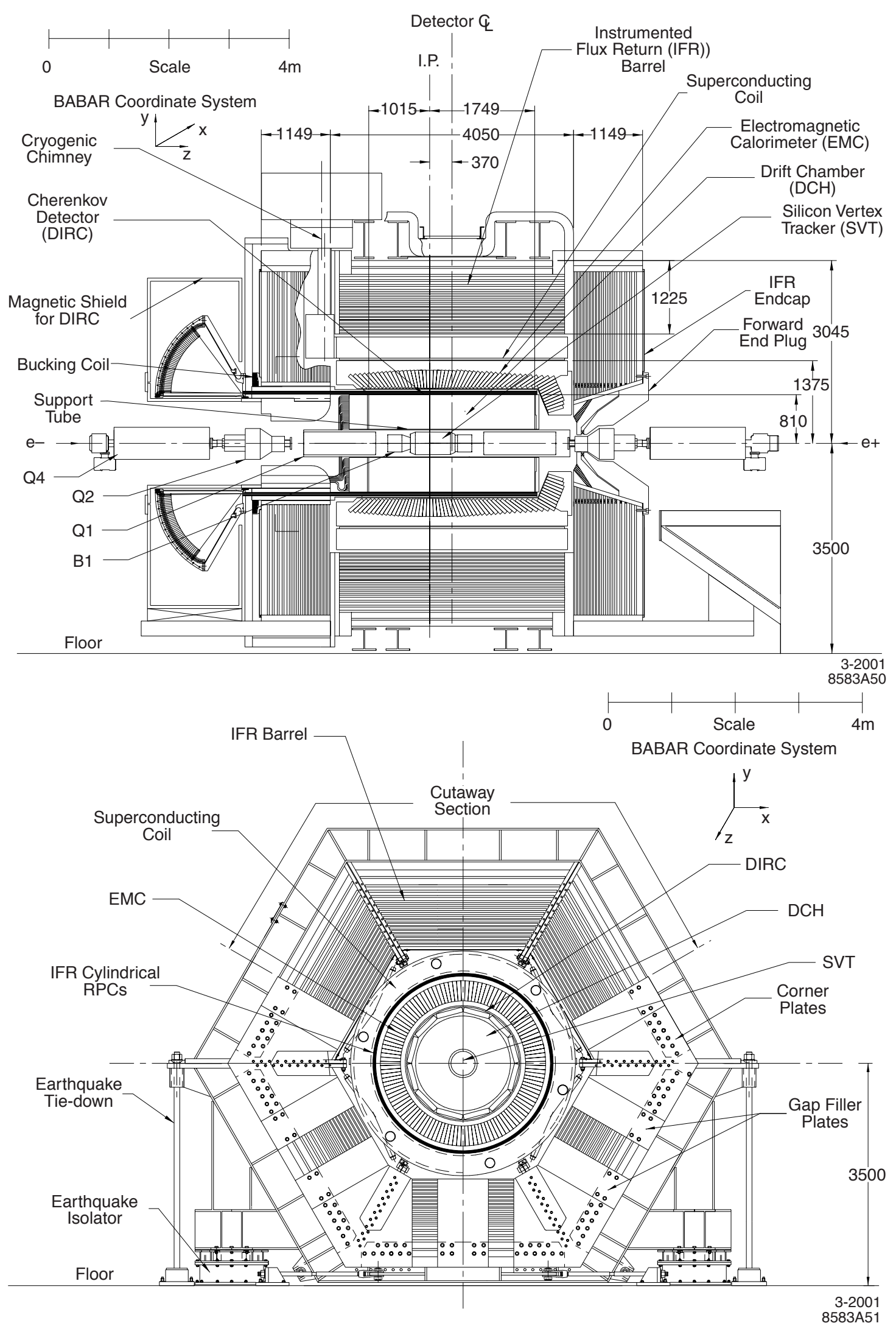

FIgURE 2.3: The BABAR detector shown in (top plot) longitudinal cross-section (bottom plot) transverse cross-section. In this figure the IR is denoted IP. 
asymmetric layout, offset from the beam-beam interaction point. Due to the Lorentz boost of the $\Upsilon(4 S)$ in the laboratory frame, this asymmetry maximises the geometric acceptance of the detector in the CM frame. The coordinate system of the BABAR detector is defined as a right handed Cartesian system; the $z$ axis is coincident with the drift chamber principal axis, the $y$ axis points upwards and the $x$ axis points away from the centre of the PEP-II storage rings. In spherical coordinates BABAR uses the American convention of $(r, \theta, \phi)$ as the distance, zenith and azimuth, respectively.

\subsubsection{Design Goals}

Physics studies using data from the BABAR experiment generally require full reconstruction of $B$ meson decays with multiple charged and neutral secondary particles. Often there is a need to identify the flavour of unreconstructed $B$ mesons produced in an event. Consequently the BABAR detector and hence each of the sub-detectors are required to fulfill a number of strict design goals [35]. The detector needs a uniform acceptance down to small zenith angles in the CM frame and a high reconstruction efficiency for long-lived charged particles with momenta as low as $60 \mathrm{MeV} / c$ and for photons with energy as low as $20 \mathrm{MeV}$. The resolution of a single $B$ decay vertex is required to be around $100 \mu \mathrm{m}$ both transverse and parallel to the $z$ axis to ensure that the reconstructed decay vertices of both $B$ mesons are sufficiently separated from each other in the laboratory frame. An efficient and accurate particle identification (PID) method for charged particles is required over a wide range of momenta. This must be sufficient to separate electrons and muons with a low probability of them being misidentified as hadrons. It must also allow for accurate identification of different hadron species, again with low misidentification probabilities.

\subsubsection{Detection of Charged Particles}

Generally when detecting the properties of long-lived charged particles one aims to measure the particle four-momentum, production vertex and trajectory followed while in the detector volume. This measurement should be non-destructive, meaning the energy transfer between the particle being measured and the detecting medium should be minimal. The BABAR detector has two sub-detectors for the measurement of such properties, a silicon strip vertex detector surrounded by a gas-filled wire drift chamber. 


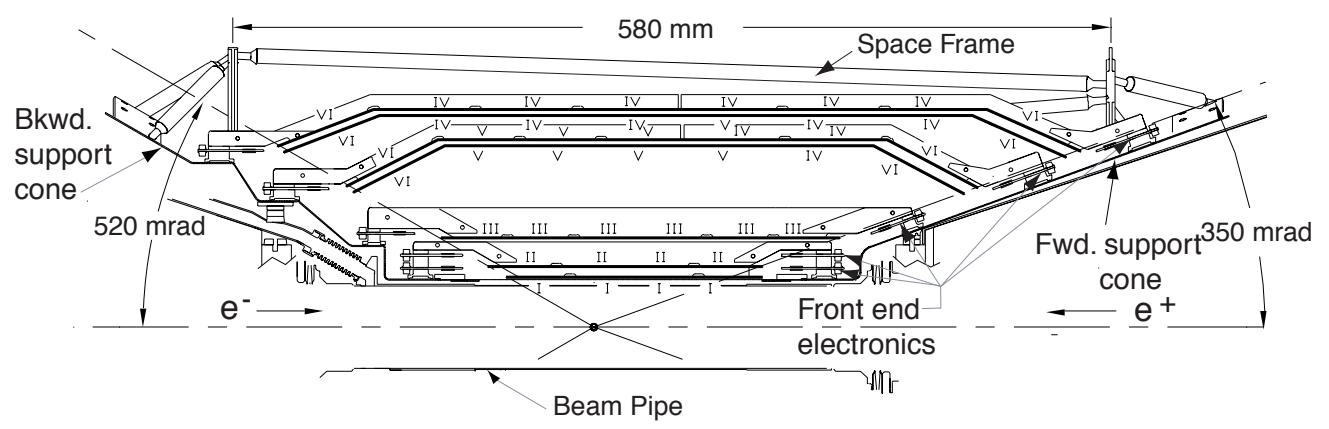

(a)

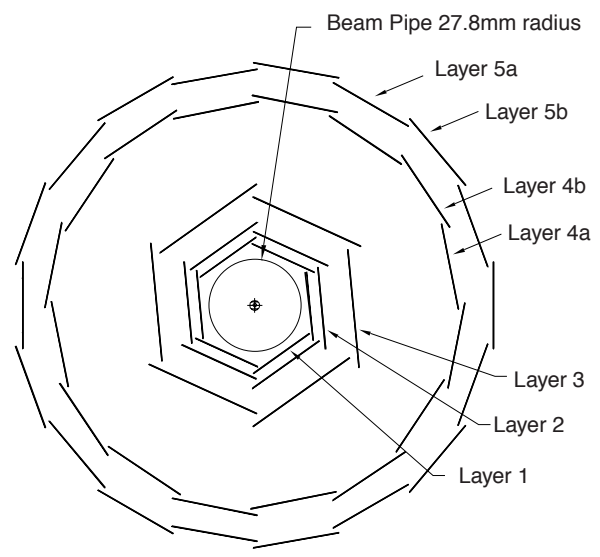

(b)

Figure 2.4: Schematic (a) longitudinal and (b) transverse sections of the BABAR silicon strip vertex detector.

\subsubsection{The Silicon Vertex Tracker}

The SVT is designed to give high spatial resolution of charged particle trajectories near the IR, thus providing the required resolution of primary particle decay vertices in a collision event. It consists of five layers of double-sided silicon strips and surrounds the $5.6 \mathrm{~cm}$ diameter beryllium beampipe which itself corresponds to $1.06 \%$ of a radiation length $\left(X_{0}\right)$. Signals are characterised by charge deposits created from ionisation in the doped silicon bulk as a charged particle traverses the detector. Longitudinal and transverse sections of the SVT are shown in figure 2.4. For each layer the inner strip of a module is orientated orthogonally to the outer strip. The former are oriented transversely to the principal axis and measure the position in $z$ of any charged particle traversing them; the latter are parallel to the principal axis and measure the position in $\phi$. From figure 2.4 it can be seen that the inner three layers, which each contain six modules, are axial in $z$ and tilted in the transverse plane, forming an overlap which ensures full radial coverage. These inner layers provide the impact parameter measurement of a charged 
particle trajectory. The outer two layers, which in order of increasing radius contain 16 and 18 modules, are arched in $z$ resulting in less material for solid angle coverage (i.e. less than if these layers had been entirely axial in $z$ ) and increasing the crossing angle of charged particles near the edge of acceptance. In the transverse plane the outer layers are divided into sub-layers at slightly different radii, again to ensure complete coverage in this plane. These layers are important in the reconstruction of trajectories of charged particles with low transverse momentum and for associating higher momentum trajectories with corresponding trajectories in the DCH. The SVT has a solid angle coverage of $90 \%$ in the CM frame and the material in the tracking volume is around $4 \%$ of $X_{0}$.

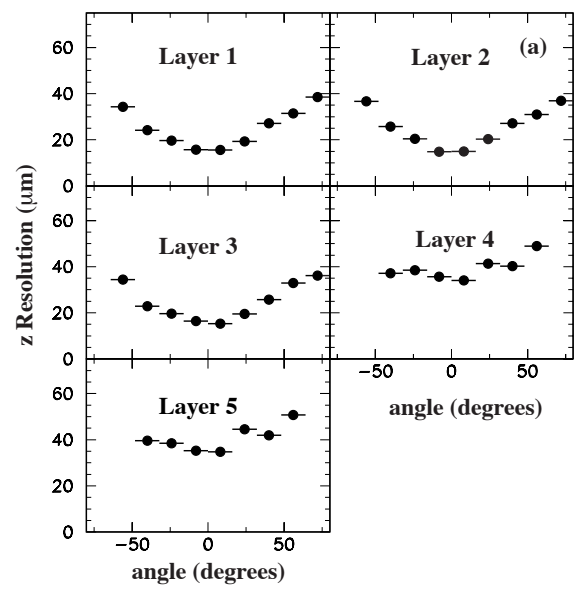

(a)

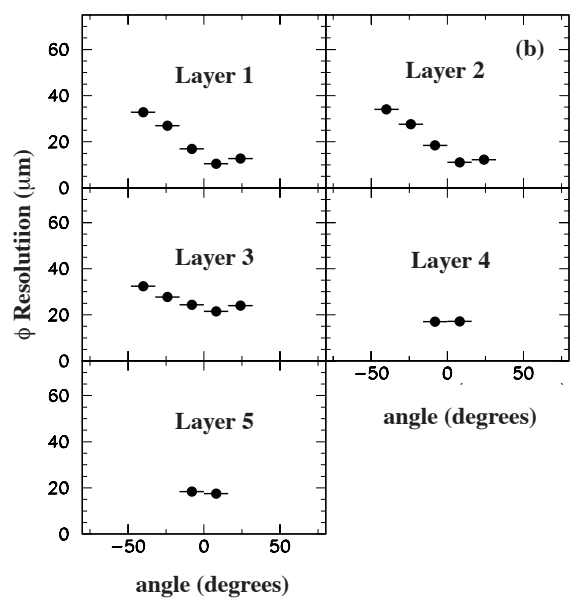

(b)

Figure 2.5: Measured resolutions in (a) $z$ and (b) $\phi$ as a function of incident track angle for charged particles traversing the SVT. The performance for each of the five layers is shown.

Figure 2.5 shows the spatial resolution performance in $z$ and $\phi$ for the SVT as a function of incident track angle. It can be seen that small resolutions ranging between around $15 \mu \mathrm{m}$ and $50 \mu \mathrm{m}$ are achieved in $z$ depending on layer and incident track angle, with the corresponding resolution for $\phi$ ranging from around $10 \mu \mathrm{m}$ to $35 \mu \mathrm{m}$. For the inner layers an asymmetry in the resolution of $\phi$ can be seen; this is due to the tilted modules in the transverse plane. The smaller incident range seen in the resolution of $\phi$ in the outer layers is also attributable to the geometry of these layers.

\subsubsection{The Wire Drift Chamber}

The DCH is designed for the efficient and high precision measurement of the momentum and angles of charged particles with momenta from around $120 \mathrm{MeV} / c$ to $4 \mathrm{GeV} / c$ and 
higher. It provides the reconstruction of any decay vertices outside the SVT, such as $K_{S}^{0}$ $\rightarrow \pi^{+} \pi^{-}$decays, and thus requires a longitudinal resolution of around $1 \mathrm{~mm}$. The average momentum of a charged particle at BABAR is less than $1 \mathrm{GeV} / c$ which means multiple scattering results in significant limitations on trajectory resolution. The DCH consists of 40 cylindrical layers of hexagonal drift cells and is filled with a 4:1 helium:isobutane gas mixture. The helium minimises multiple scattering and has a short drift time, while the isobutane absorbs any photons produced by ionised particles to prevent secondary ionisations from the photoelectric effect. Each cell contains a gold-plated tungstenrhenium sense wire, held at a typical operating voltage of $1960 \mathrm{~V}$ and surrounded by six grounded gold-plated aluminium field wires. Charged particles traversing a cell ionise the gas resulting in a charge avalanche on the sense wire. At operating voltages the typical gain in a cell from an avalanche is around $5 \times 10^{4}$. The hexagonal layout ensures a close to circular field across the majority of a cell.

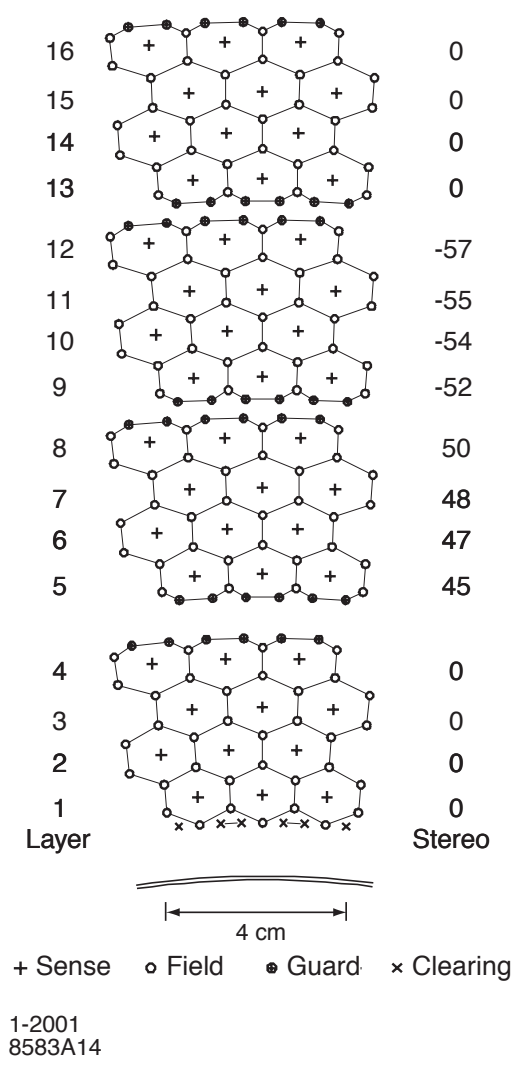

FIGURE 2.6: Schematic representation of the first four superlayers in the DCH. The stereo angle in mrad shows the AUVA structure used for longitudinal measurement.

The 40 layers of the DCH are split into 10 'superlayers', each of four layers. The first four superlayers are shown in figure 2.6. To obtain longitudinal position measurements six of the 10 superlayers are tilted relative to the $z$ axis though a given stereo angle. The stereo angles alternate between axial $(\mathrm{A})$ and stereo $(\mathrm{U}, \mathrm{V})$ pairs where $\mathrm{U}$ has a positive 
stereo angle and $\mathrm{V}$ negative. The resultant order of superlayers is AUVAUVAUVA. Transversely the gas mixture and wires of the DCH correspond to $0.2 \%$ of $X_{0}$; this increases to $1.08 \%$ when the inner and outer support walls are included.

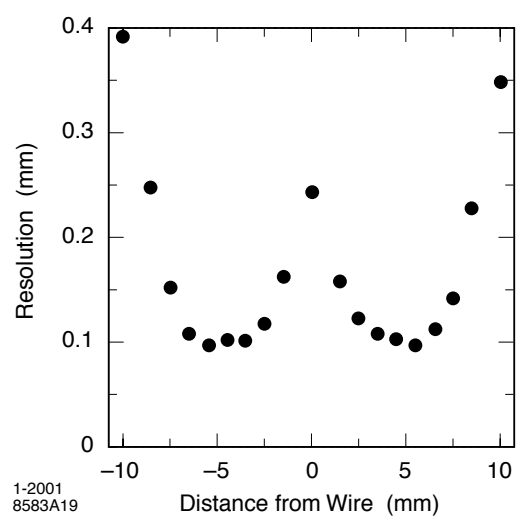

FIGURE 2.7: The average resolution of cells in layer 18 of the DCH as a function of distance from the sense wire.

The electronic readout from the DCH is designed to measure the drift time and integrated charge for every wire with a signal. Primary ionisation cluster positions are determined from timing of the leading edge of the amplified signal with a designed position resolution of $140 \mu \mathrm{m}$ averaged over cells in the transverse plane. Figure 2.7 shows the average position resolution for multi-hadron events as a function of drift distance in layer 18 for tracks to the left and right of a sense wire. The resolution deteriorates closer to the wire due to insufficient charge build up from the avalanche and again further from the wire due to timing uncertainties resulting from the diffusion of the avalanched charge. The average resolution for the cell is close to design.

\subsubsection{Charged Particle Trajectory Reconstruction}

Both the SVT and the DCH are immersed in a uniform $1.5 \mathrm{~T}$ magnetic field directed along the $z$ axis from the superconducting solenoid. Due to the resultant Lorentz force a charged particle will follow a helical path in the transverse plane, the radius of curvature (RoC) of which gives a measurement of the transverse momentum magnitude, $p_{T}$. The direction of the RoC determines whether the particle holds a positive or negative charge. A high resolution measurement of the momentum of a charged particle therefore requires an accurate reconstruction of its trajectory. BABAR achieves this through complimentary measurements from the SVT and DCH. Each detector makes a position measurement in a single active element of its volume known as a 'hit'. Reconstructed trajectories, known as 'tracks', are created from pattern recognition software which performs a Kalman fit 
on a collection of individual hits to determine if they are consistent with the passage of a single charged particle. The fit algorithm corrects for measured inhomogeneities in the magnetic field, although systematic uncertainties remain as the field was mapped in the absence of PEP-II magnets. Reconstructed tracks are described by five parameters, $\left(d_{0}, z_{0}, \phi_{0}, \tan \lambda, \omega\right)$, all measured at the point of closest approach (POCA) to the $z$ axis; $d_{0}$ and $z_{0}$ are the distances from the POCA to the transverse plane and the $z$ axis respectively, $\phi_{0}$ is the azimuthal angle, $\lambda$ the dip angle relative to the transverse plane, and $\omega$ is the track curvature given as $1 / p_{T}$.

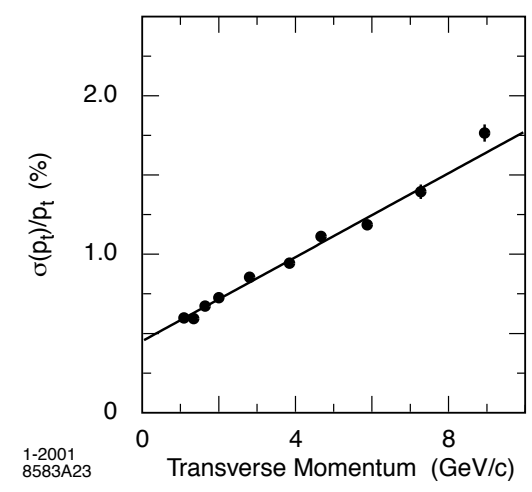

FIGURE 2.8: The combined resolution of the SVT and DCH in transverse momentum for cosmic muons.

Figure 2.8 shows the combined $p_{T}$ resolution of the SVT and DCH for cosmic muons. The resolution is clearly linear in $p_{T}$ and the corresponding fitted line gives a resolution function,

$$
\sigma_{p_{T}} / p_{T}=(0.13 \pm 0.01) \% \times p_{T}+(0.45 \pm 0.03) \% .
$$

Tracks which pass the Kalman fitter selection are available through the BABAR computing framework for event reconstruction in a physics analysis.

\subsubsection{Charged Hadron Identification}

The SVT and DCH measure spatial momentum of reconstructed charged particle tracks. However, to fully reconstruct the four-momentum the mass of that particle must also be known. The BABAR detector is required to differentiate between the properties of different charged hadrons, in particular pions, kaons and protons. PID for long-lived charged leptons is discussed in section 2.2.5.

Ionisation energy deposited in the tracking sub-detectors is particularly important for low momentum tracks. Characteristic Bethe-Bloch curves [14] show distributions of ionisation energy deposited by a charged particle, $\mathrm{d} E / \mathrm{d} x$, as a function of momentum 
and can thus indicate which particle traversed the tracking medium. Figure 2.9 shows

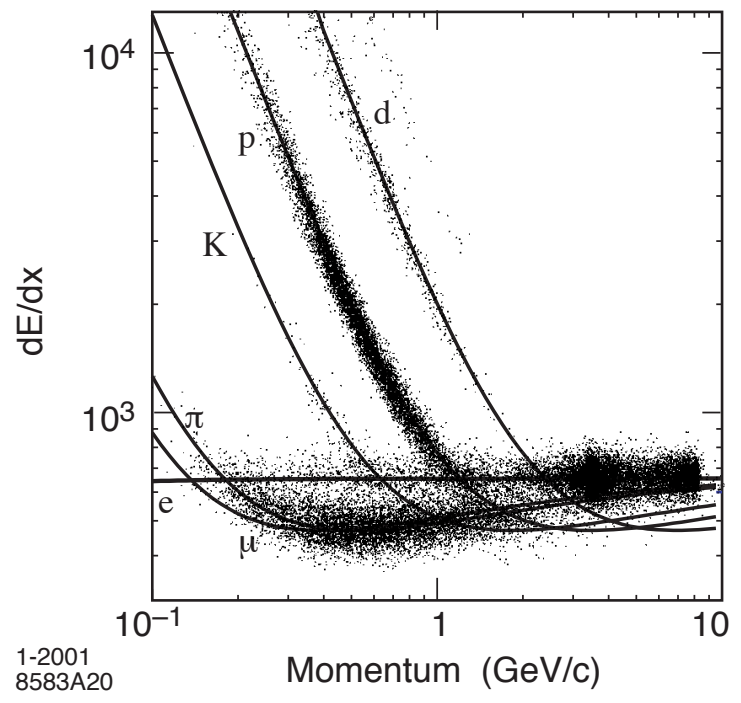

Figure 2.9: $\mathrm{d} E / \mathrm{d} x$ as a function of track momentum comparing Bethe-Bloch predictions to data for various particle types. The unit of the $\mathrm{d} E / \mathrm{d} x$ curve is arbitrary due to corrections for the deposited charge in individual DCH cells.

the measurement of $\mathrm{d} E / \mathrm{d} x$ in the $\mathrm{DCH}$ as a function of momentum and compares data to the Bethe-Bloch hypothesis for different particle types. It can be seen that for low momentum tracks in the $1 / \beta^{2}$ region a good separation exists between kaons, pions and protons. As particles reach the minimum ionisation energy and approach the relativistic limit the separation worsens. The resolution of the $\mathrm{DCH}$ in $\mathrm{d} E / \mathrm{d} x$ is around $7 \%$ which gives good separation of pions and kaons up to momenta of $700 \mathrm{MeV} / c$. PID measurements from the tracking system are complementary to those from the Cherenkov detector (described below); however, charged particles with $p_{T}<180 \mathrm{MeV} / c$ will not reach the DIRC so the tracking volume provides the only measure of PID. Furthermore tracks with even lower transverse momentum will only deposit energy in the SVT which has a $\mathrm{d} E / \mathrm{d} x$ resolution of around $14 \%$. This allows a $2 \sigma$ separation of kaons and pions up to $500 \mathrm{MeV} / c$ in this sub-detector alone.

Generally the PID algorithms for charged tracks at BABAR combine relevant information from different sub-systems into custom PID selectors which maximise the probability of correct identification. These selectors are available to analysts during event reconstruction and are described in section 3.2.5. 


\subsubsection{The Cherenkov Detector}

It can be seen from figure 2.9 that the ability of the DCH to distinguish between charged particle species with momenta above $700 \mathrm{MeV} / c$ deteriorates rapidly. Many physics processes studied with $B A B A R$, including the analysis presented in subsequent chapters, require good separation between reconstructed pions and kaons up to momenta of $4 \mathrm{GeV} / c$. It is for this reason that $B A B A R$ employs a sub-detector dedicated to charged particle PID, the DIRC. Charged particles with velocity $\beta=p / E$ will emit Cherenkov radiation in a cone of opening angle $\cos \theta_{c}=1 / \beta n$ if $\beta>1 / n$, where $n$ is the refractive index of the medium being traversed. It follows that given the particle momentum and angle, which in $B A B A R$ is given by the tracking system, a measurement of $\theta_{c}$ will give the particle mass. The DIRC is intended to exploit this principle but must avoid degrading the energy resolution of the EMC which lies beyond it. For this reason the detection mechanism for Cherenkov photons is outside the active detector volume.

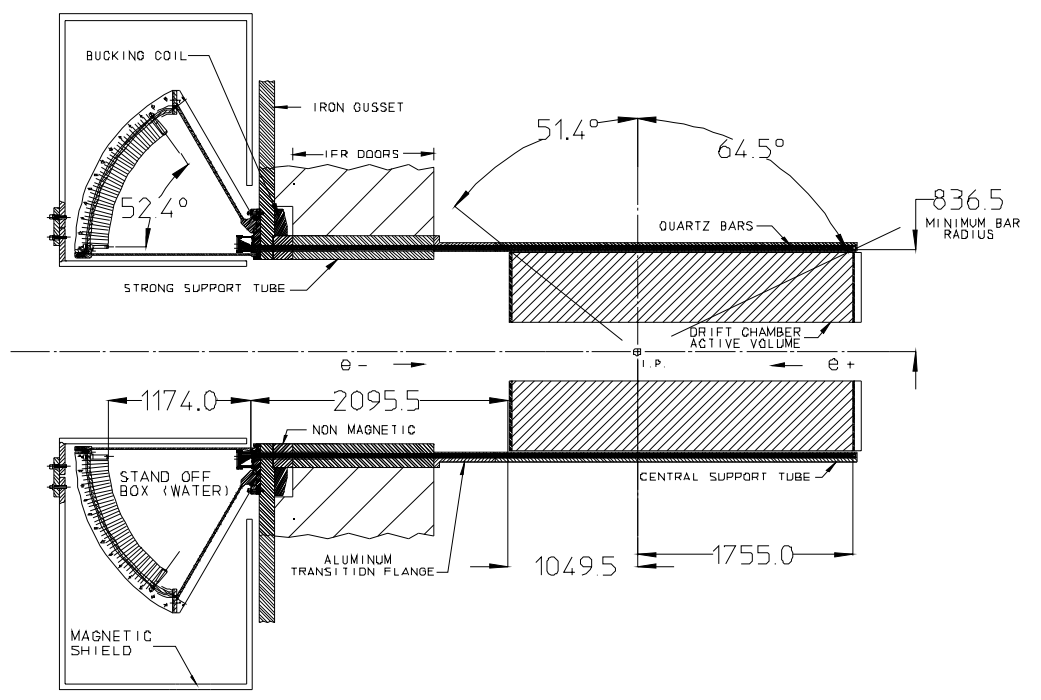

FIGURE 2.10: Longitudinal section of the DIRC showing the active medium and external detection system.

The DIRC is shown in longitudinal section in figure 2.10. It consists of 144 fused silica bars, with $n=1.473$, which act as the radiative material and also transfer the emitted photons by total internal reflection to the rear end of the detector, preserving $\theta_{c}$ (forward going photons are reflected by a mirror). Here they enter a stand-off box (SOB) filled with purified water whose refractive index is close to that of the quartz bars to reduce internal reflection and refraction at the interface. The SOB is mounted with 10,752 photomultiplier tubes to detect the Cherenkov light which forms a conic section whose opening angle is $\theta_{c}$, once corrected for the effects of refraction. 


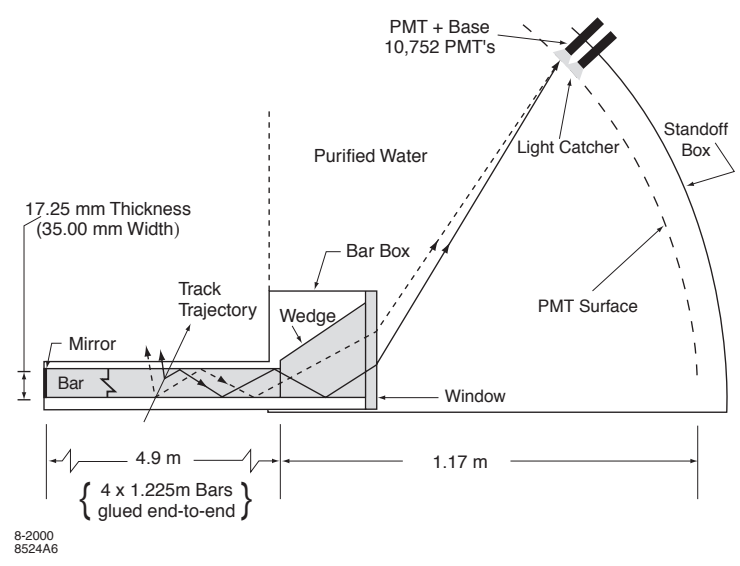

FIGURE 2.11: Schematic showing the principal of operation for the DIRC.

Figure 2.11 shows a schematic demonstrating the operating principle of the DIRC as described above. It should be noted that this schematic is an oversimplification showing the operation in only two dimensions of what is a three dimensional system. Transversely

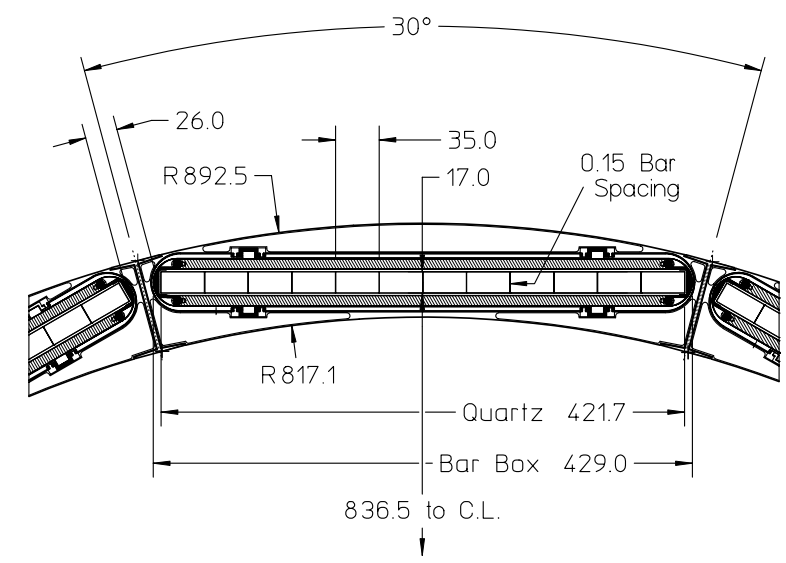

FIGURE 2.12: A transverse section of one of the 12 polygonal sides of the DIRC barrel.

the DIRC bars are arranged in a 12-sided polygonal barrel, with each side containing 12 bars as shown in figure 2.12. The material in the detector volume corresponds to $17 \%$ of $X_{0}$ and due to the polygonal structure the acceptance in the azimuth is around $94 \%$. In the CM frame the acceptance is around $83 \%$ of the polar angle cosine.

Figure 2.13 (a) shows the expected separation of pions and kaons as a function of momentum for $B^{0} \rightarrow \pi^{+} \pi^{-}$events inferred from the measured Cherenkov angle resolution and number of Cherenkov photons per track in dimuon events. It can be seen that the separation varies from greater than $10 \sigma$ at $2 \mathrm{GeV} / c$ to less than $3 \sigma$ at $4 \mathrm{GeV} / c$. Figure 2.13 (b) shows the efficiency and misidentification probability using the DIRC for selection of charged kaons from a $D^{0} \rightarrow K^{+} \pi^{-}$control sample. The average kaon selection 


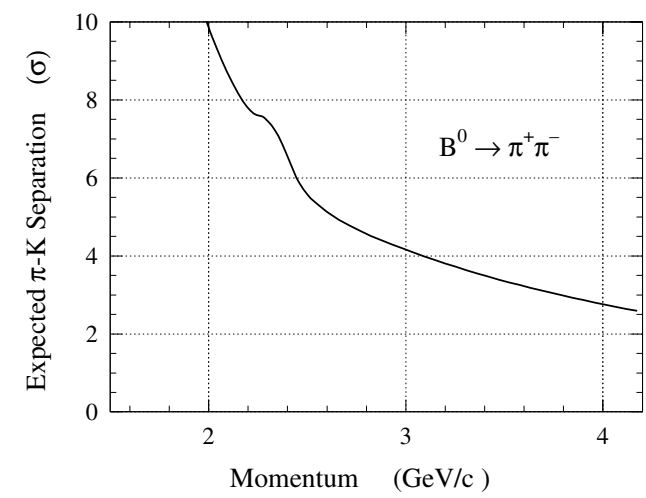

(a)

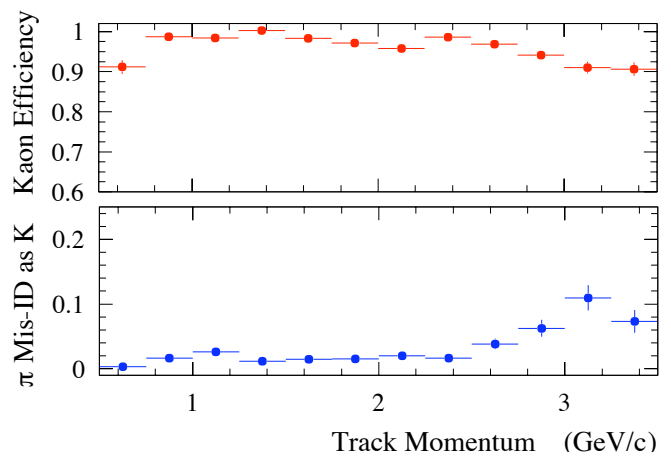

(b)

FIGURE 2.13: DIRC expected separation of pions and kaons for (a) $B^{0} \rightarrow \pi^{+} \pi^{-}$events and (b) the measured kaon efficiency and pion misidentification in $D^{0} \rightarrow K^{+} \pi^{-}$events.

efficiency and pion misidentification are $96.2 \pm 0.2 \%$ and $2.1 \pm 0.1 \%$ respectively, where the errors are statistical.

\subsubsection{Photon Detection}

Of particular importance to many physics studies with BABAR data is the accurate measurement of both the angle and energy of photons. Such measurements are required to be highly efficient with excellent resolution of photon energies ranging from $20 \mathrm{MeV}$ to $9 \mathrm{GeV}$. The lower bound is set by the requirement for efficient reconstruction of both $\pi^{0} \rightarrow \gamma \gamma$ and $\eta \rightarrow \gamma \gamma$ decays. The upper bound relates to the need to measure QED processes such as $e^{+} e^{-} \rightarrow \gamma \gamma$ and $e^{+} e^{-} \rightarrow e^{+} e^{-}(\gamma)$ for calibration and luminosity determination. In the presented analysis photons with energies of up to $4.5 \mathrm{GeV}$ in the laboratory frame are required for event reconstruction and many of the $B$ meson decays to be considered require the reconstruction of at least one $\pi^{0} \rightarrow \gamma \gamma$ or $\eta \rightarrow \gamma \gamma$ decay. The measurement of photon energies and angles at BABAR is carried out with an electromagnetic calorimeter.

\subsubsection{The Electromagnetic Calorimeter}

The EMC is a hermetic total absorption calorimeter finely segmented with 6580 thalliumdoped caesium iodide $(\mathrm{CsI}(\mathrm{Tl}))$ scintillating crystals. Geometrically it is split into a cylindrical barrel and forward endcap giving it full coverage in the azimuth and $90 \%$ solid angle coverage in the CM frame. The barrel contains 5760 crystals arranged into 48 rings of 120 crystals, while the endcap has eight rings in total of which, in order of decreasing zenith angle, three contain 120 crystals, three contain 100 crystals and two 


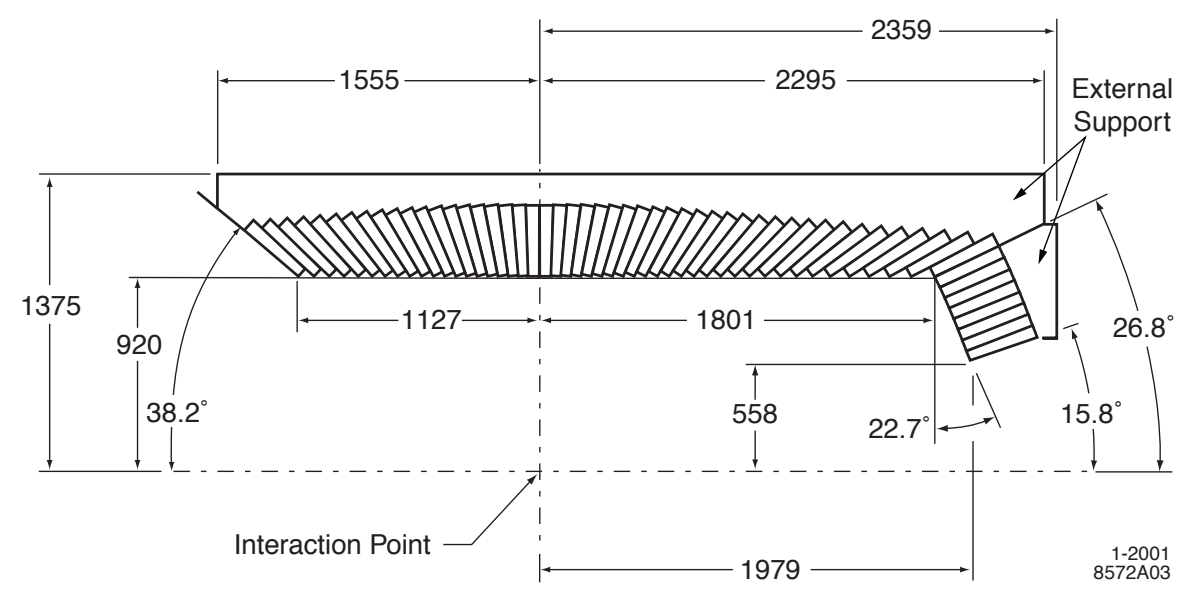

Figure 2.14: A longitudinal section showing the top half of the EMC which indicates the crystal layout in the barrel and endcap sections. All dimensions are in $\mathrm{mm}$.

contain 80 crystals. $\mathrm{CsI}(\mathrm{Tl})$ has a high light yield and small Molière radius giving high energy and angular resolution as well as a $X_{0}$ of $1.85 \mathrm{~cm}$ to ensure shower containment. Crystal lengths vary from $16 X_{0}$ in the backward barrel region at high zenith to $17.5 X_{0}$ in the forward barrel and endcap to minimise leakage from higher energy particles moving in the boost direction.

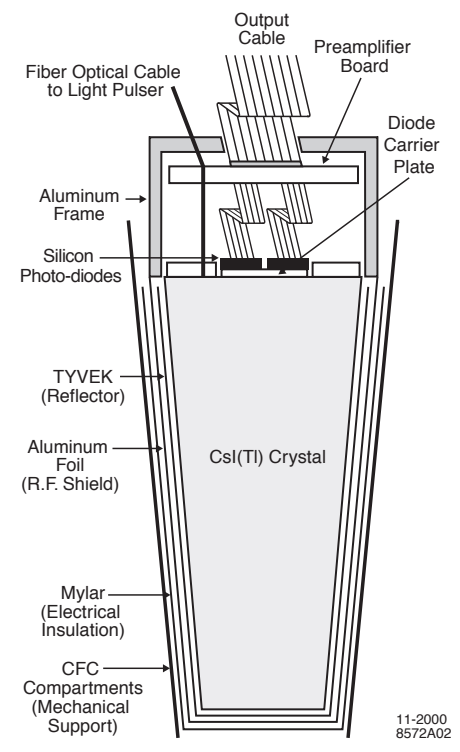

Figure 2.15: Schematic representation of a $\mathrm{CsI}(\mathrm{Tl})$ scintillating crystal used in the BABAR EMC.

As shown in figure 2.15 crystals are trapezoidal in shape. They guide the scintillating light, which is proportional to the deposited energy, through total internal reflection from highly polished surfaces to a pair of silicon diodes used for detection. To reduce transmission an additional two layers of diffuse white reflector surround the crystal edges. 
The front face area of crystals varies to achieve hermicity but is typically $4.7 \times 4.7 \mathrm{~cm}^{2}$, close to the Molière radius to maximise the angular resolution while limiting the required number of crystals. Readout diodes are mounted on the back face of each crystal via an optical epoxy to maximise light transmission. Signals are amplified and digitised in the detector volume and then passed out via fibre optical cables.

\subsubsection{Calorimeter Resolution}

The energy resolution of a homogeneous crystal calorimeter is given by summing the following terms in quadrature,

$$
\frac{\sigma_{E}}{E}=\frac{a}{\sqrt[4]{E / \mathrm{GeV}}} \oplus b
$$

where $E$ and $\sigma_{E}$ are the respective energy and corresponding RMS error of a detected photon. The $a$ term is an energy dependent variable primarily describing fluctuations in photon statistics, but is also affected by noise from both beam-generated background and electronics. The $b$ term, which dominates at energies $>1 \mathrm{GeV}$, accounts for nonuniformity in light collection, leakage and absorption in the detector and calibration uncertainties.

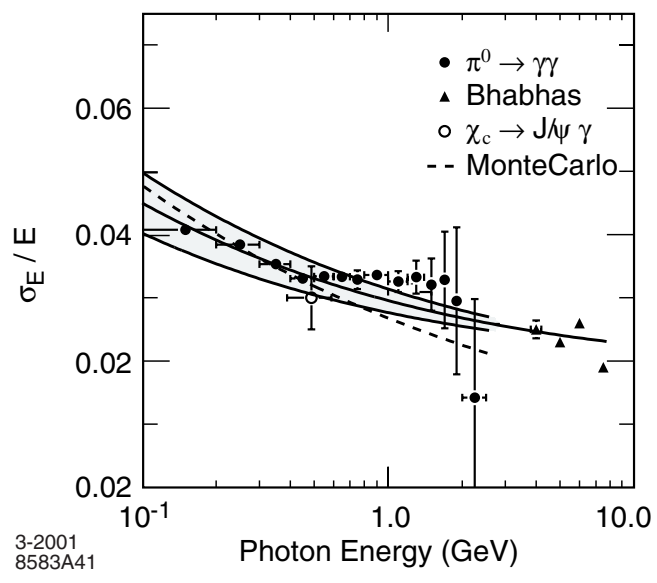

Figure 2.16: EMC energy resolution in data for different processes. The central solid line represents the fit used to extract the resolution measurement while the shaded region represents $\pm 1 \sigma$ bands.

The EMC measures low energy resolutions directly with a radioactive source ${ }^{4}$ giving $\sigma_{E} / E=5.0 \pm 0.8 \%$ at $6.13 \mathrm{MeV}$. High energy resolutions are calculated from Bhabha scattering events, where energy can be predicted from the $e^{-}$zenith angle, and gives $\sigma_{E} / E=1.9 \pm 0.1 \%$ at $7.5 \mathrm{GeV}$. Figure 2.16 shows EMC energy resolutions for a variety

\footnotetext{
${ }^{4}$ Through the reaction ${ }^{16} O^{*} \rightarrow{ }^{16} O+\gamma$.
} 
of processes. The fitted resolution gives $a=2.32 \pm 0.30 \%$ and $b=1.85 \pm 0.12 \%$ which is in reasonable agreement with Monte Carlo studies of the expected resolution.

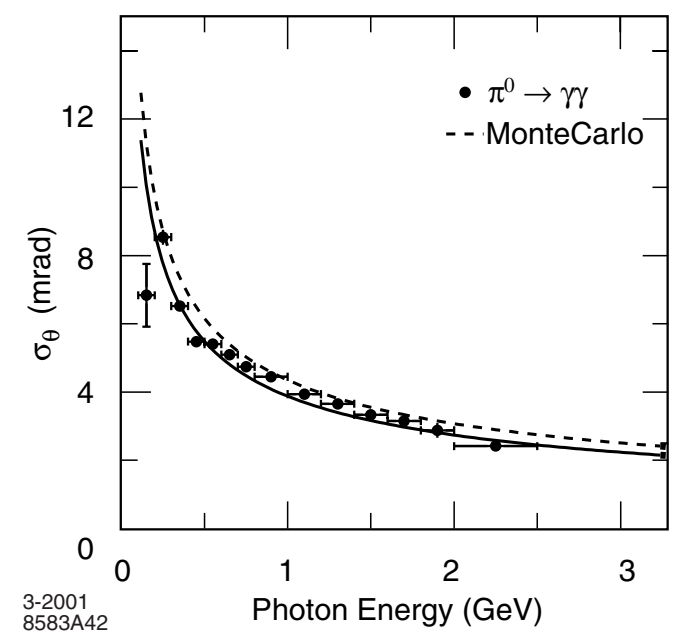

Figure 2.17: EMC angular resolution as measured from $\pi^{0} \rightarrow \gamma \gamma$ decays. The solid line shows the fit used to extract the resolution.

The angular resolution depends on the transverse crystal size and distance from the IR and is empirically parameterised as the sum of an energy dependent term, $c$, and a constant term, $d$ :

$$
\sigma_{\theta}=\sigma_{\phi}=\frac{c}{\sqrt{E / \mathrm{GeV}}}+d
$$

Angular resolution is measured from $\pi^{0}$ and $\eta$ decays to photons of approximately equal energy. Figure 2.17 shows the results for $\pi^{0}$ decays with angular resolution as a function of energy. The fitted line is of the empirical form of (2.3) and gives $c=3.87 \pm 0.07 \mathrm{mrad}$ and $d=0.00 \pm 0.04 \mathrm{mrad}$ which is slightly better than expected from Monte Carlo studies.

\subsubsection{Calorimeter Energy Clusters}

Electromagnetic showers in the EMC are typically spread over many crystals. Pattern recognition software is used to efficiently identify these clusters and then perform a search for individual local maxima known as 'bumps'. This differentiates between clusters from a single source and those formed from overlapping showers. Clusters are required to have at least one crystal with an energy deposit greater than $10 \mathrm{MeV}$ and adjacent crystals are included if they have an energy above $1 \mathrm{MeV}$ or if they have a neighbour with energy exceeding $3 \mathrm{MeV}$. The total energy of a cluster must be in excess of $20 \mathrm{MeV}$. A weighted iterative algorithm calculates the energy associated with each bump in a cluster and the respective angular position is calculated with a centre-of-gravity method 
using logarithmic weights. Finally reconstructed tracks are projected onto the inner face of the EMC to determine if a particular bump centroid is associated with a charged particle. This process is known as 'track-matching'; if no associated tracks are found the bump is assumed to originate from a neutral particle. For all clusters a correction is made to the measured energy to account for energy leakage due to the gaps between crystals.

Many useful parameters can be calculated for a bump of $n$ crystals in the EMC [39]. Two of these quantities are directly relevant to the analysis in this thesis.

The lateral moment, $L_{b u m p}$, is defined as,

$$
L_{\text {bump }}=\frac{\sum_{i=3}^{n} E_{i} r_{i}^{2}}{\left(\sum_{i=3}^{n} E_{i} r_{i}^{2}\right)+\left(E_{1}+E_{2}\right) r_{0}^{2}},
$$

where $E_{i}$ is the energy of the $i$ th crystal ${ }^{5}$ in the bump, $r_{i}$ is the distance between the corresponding crystal and bump centroid and $r_{0}=5 \mathrm{~cm}$ is the average distance between two crystal front-faces. Electromagnetic showers (e.g. from an electron or photon) tend to deposit a large fraction of their energy within a few crystals whereas hadronic showers typically have a larger energy spread. It follows that hadronic showers will on average have a larger lateral moment.

The second moment, $S_{\text {bump }}$, is defined as,

$$
S_{\text {bump }}=\frac{\sum_{i} E_{i} \Delta \alpha_{i}^{2}}{\sum_{i} E_{i}},
$$

where $\Delta \alpha_{i}$ is the angle between the crystal and the bump centroid. Merged $\pi^{0}$ decays with a single local maximum in the EMC will generally have an elliptical energy distribution whereas a high energy photon will have an energy distribution symmetric about the centroid. The energy deposit from a high energy photon will therefore have a lower second moment associated with it.

\subsubsection{Electron and Muon Identification}

The identification of long-lived charged leptons is important for determining the flavour of semileptonic $B^{0}$ and $\bar{B}^{0}$ meson decays, for the reconstruction of $\tau$ decays and for the study of QED processes such as $e^{+} e^{-} \rightarrow \mu^{+} \mu^{-}$. The tracking system and DIRC can provide some information for such PID as discussed above. Electron PID can also make use of EMC information; generally an electron will deposit all of its energy in an EMC

\footnotetext{
${ }^{5}$ Crystal numbering is energy ordered such that the most energetic crystal is labelled 1 , the next energetic crystal labelled 2 and so on.
} 
shower and due to its relatively low mass the ratio $E / p$ will be close to one, which is not the case for more massive charged hadrons. Muons tend to pass through the EMC as a minimum ionising particle (MIP) and traverse the steel solenoid flux return. To assist in the separation of charged hadrons from muons the flux return is instrumented to detect any ionisation from traversing muons.

\subsubsection{The Instrumented Flux Return}

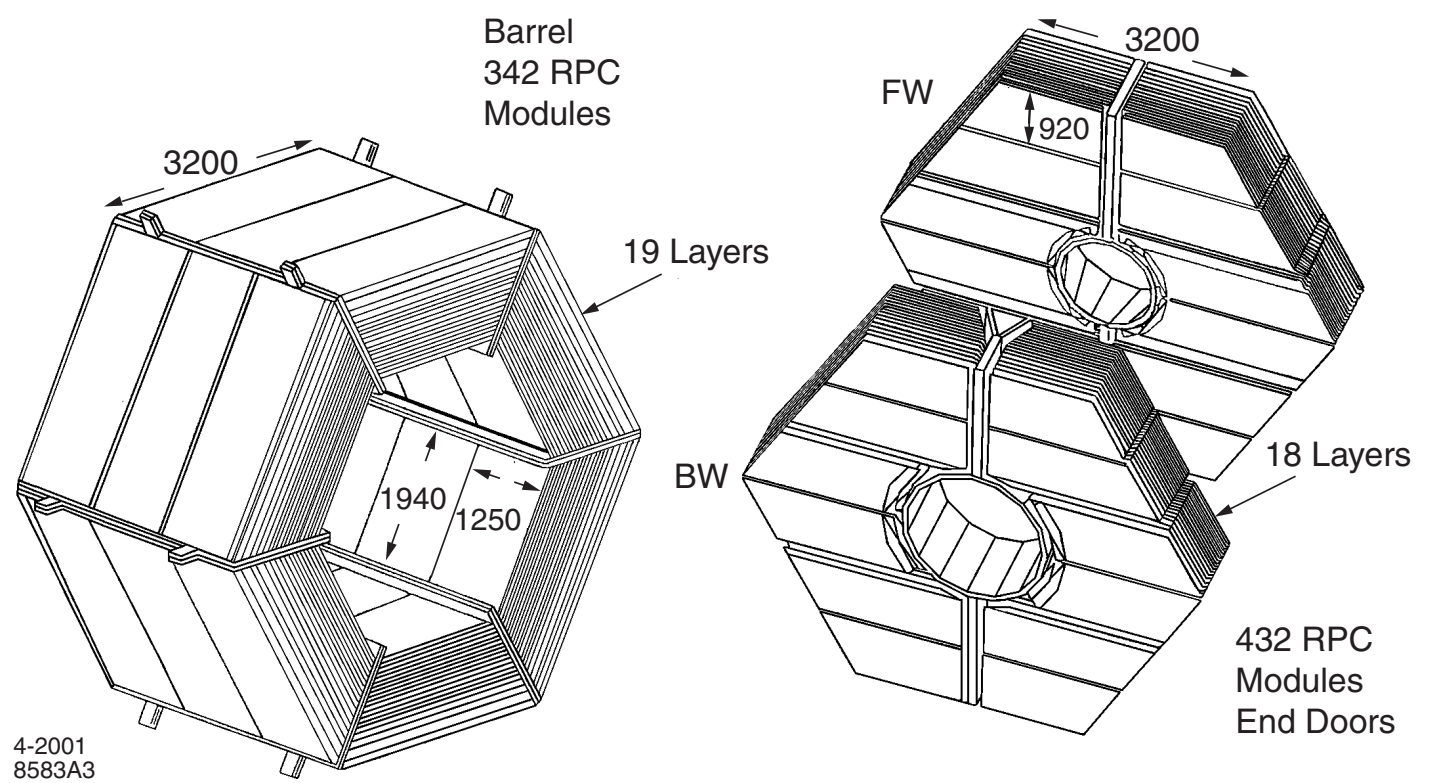

FiguRE 2.18: Layout of the IFR showing the segmented steel and instrumented layers for the barrel and two end doors.

The IFR makes use of the BABAR steel flux return as a muon filter and hadron absorber. Gaps between the finely segmented sections of the steel are instrumented to detect streamers from ionising particles. Figure 2.18 shows the layout of the IFR which consists of a hexagonal barrel with 19 layers of instrumentation and two end doors with 18 layers. The depth of steel layers varies from $2 \mathrm{~cm}$ at the inner layers to around $10 \mathrm{~cm}$ at the outer layers. Initially the IFR was instrumented with 806 single gap resistive plate chambers (RPCs) with 57 in each of the six barrel sectors and 108 in each of the four half end doors. An additional two cylindrical layers of $32 \mathrm{RPCs}$ were installed between the EMC and solenoid to detect particles exiting the EMC.

Figure 2.19 shows a schematic cross-section of an IFR RPC. The active volume is filled with a mixture of argon, freon and isobutane which creates a discharge on the passage of an ionising particle. The discharge is detected via two capacitive readout strips which are placed orthogonally to measure both $\phi$ and $z$ of the ionisation. In the first years 


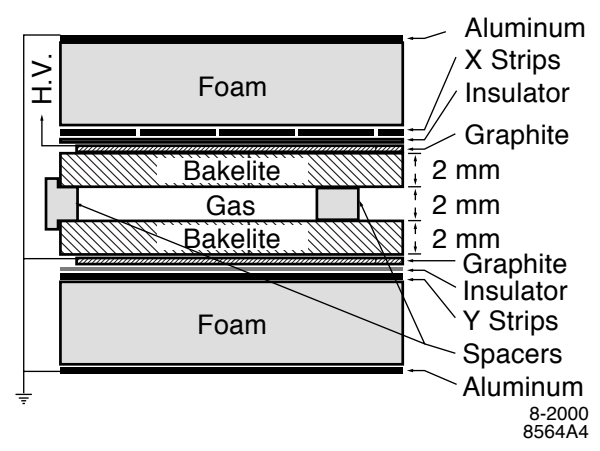

FIGURE 2.19: Section showing the principal RPC design.

of operation a serious degradation in RPC detection efficiency was observed due to a number of factors including construction flaws. This led to the barrel instrumentation being replaced with limited streamer tubes (LSTs) [40]. Of the six barrel segments, RPCs in the top and bottom were replaced between Run 4 and Run 5 while those in the remaining four segments were replaced between Run 5 and Run 6 . The end doors were instrumented with RPCs for the lifetime of the experiment although some modules were replaced with a slightly modified design.

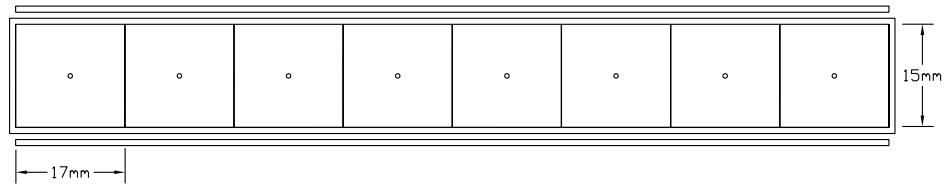

FIGURE 2.20: Section showing the principal LST design.

Figure 2.20 shows an IFR LST sketched in transverse cross section. Each cell has a single gold-plated anode wire at high voltage, typically around $5500 \mathrm{~V}$, and contains a gas mixture of $\mathrm{CO}_{2}$, argon and isobutane (to prevent secondary ionisations) in a (89:3:8) ratio. Ionising particles cause a discharge in the gas which is read out from the wire, giving a $\phi$ coordinate of the discharge. A simultaneous charge is induced on a plane mounted below the tube consisting of 96 copper strips perpendicular to the wire direction which read out the $z$ coordinate along the $4 \mathrm{~m}$ length of the plane. The first LST installation fitted $24 z$-planes and 388 tubes, while the second stage installed 48 $z$-planes and 776 tubes.

Muon detection efficiency as a function of the pion rejection efficiency in the IFR barrel for high energy muons is shown in figure 2.21. The deterioration in RPC performance between 2000 and 2004 can clearly be seen. LST segments installed for Run 5 significantly improve the muon detection efficiency compared to the remaining barrel RPCs. 


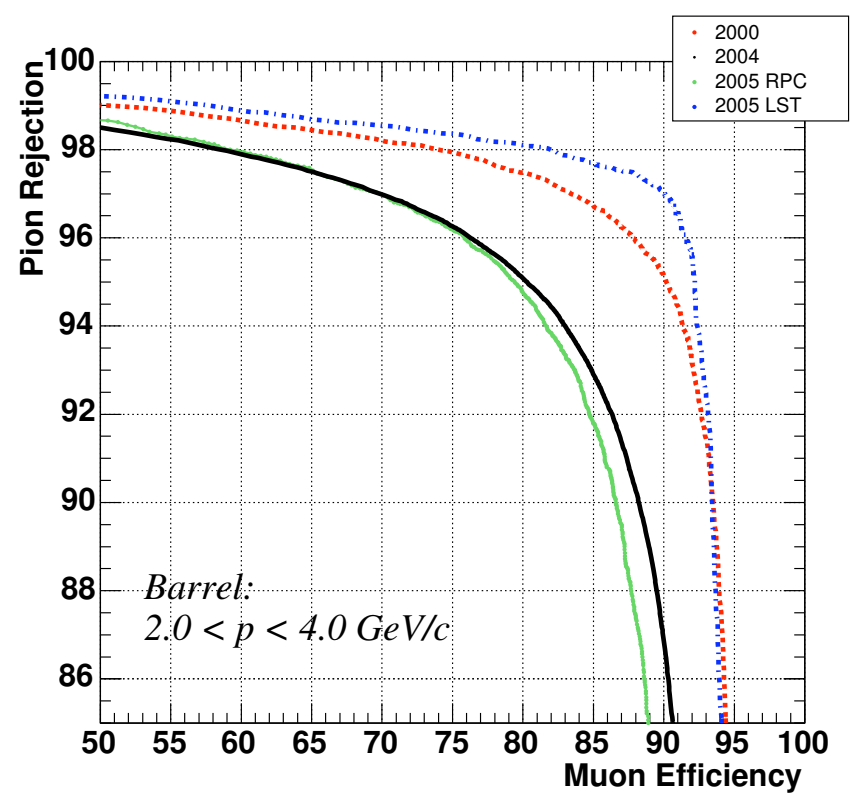

FiguRe 2.21: Muon detection efficiency as a function of pion rejection efficiency for high energy muons in the IFR barrel. The dotted line shows RPC efficiencies in 2000, the upper and lower solid lines show the RPC efficiencies in 2004 and 2005 respectively.

The dotted-dashed line shows the efficiency for LSTs installed for Run 5 in 2005.

In their first year of operation the LSTs even out-performed the original efficiency of the RPCs.

\subsubsection{The Trigger and Data Acquisition System}

The BABAR trigger is designed to manage the readout rate of detector sub-systems, whose latency buffers store signals from all $e^{+} e^{-}$interactions. The required rate is determined by the bandwidth at which data can be transferred and stored before a more detailed offline reconstruction and analysis can be performed. The trigger employs a two level hierarchy: a 'Level 1' hardware trigger (L1T) with input from the DCH, EMC and IFR and a 'Level 3' software trigger (L3T) which performs real time event reconstruction and classification. The combination of this hierarchy is designed to be over $99 \%$ efficient in the identification and readout of $B \bar{B}$ events as well as at least $95 \%$ efficient for continuum events and $90-95 \%$ efficient for $\tau^{+} \tau^{-}$events. The system is also designed to be redundant in its event identification to allow the accurate study of trigger efficiencies. 


\subsubsection{The Hardware Trigger}

The L1T monitors a constant stream of information from the DCH and EMC in the drift chamber trigger (DCT) and electromagnetic calorimeter trigger (EMT) respectively. The IFR also provides information to allow for triggering of cosmic ray and $\mu^{+} \mu^{-}$events, mainly for diagnostics. The L1T is entirely digital using reduced data derived from the respective subsystems.

Input to the DCT consists of one bit for each of the $7104 \mathrm{DCH}$ cells conveying time information derived from the sense wire of that cell. Groups of hits in adjacent DCH cells are identified by a track segment finder. This information is passed to a binary link tracker which groups segments into tracks. Tracks recognised by the DCT are then used to form trigger primitives depending on whether they satisfy predefined criteria including reaching a given superlayer, exceeding a $p_{T}$ threshold or satisfying a cut in $z$.

The EMT divides the EMC into 280 towers in a $7 \times 40$ array in $(\theta, \phi)$ of which the barrel contributes $6 \times 40$ towers and the endcap $1 \times 40$. Each tower in the barrel consists of 24 crystals in a $8 \times 3$ array, while the endcap towers are formed of wedges in $\phi$ containing between 19 and 22 crystals. The energy from each tower in $\theta$ is summed independently for the $40 \phi$ sectors. Neighbouring $\phi$ energy strips are then added together to identify any showers which may cross two adjacent $\phi$-sectors. The resultant energy for all 40 strips are compared to predefined thresholds to form trigger primitives. The sums are then ORed between neighbouring $\phi$ sectors giving a 20-bit $\phi$ map for each primitive.

Primitives from the DCT and EMT are passed in parallel to the global level trigger (GLT) as bitmaps in $\phi$. The GLT compares these bitmaps to 24 predefined physics signatures. If the GLT finds a match it passes this information to the Fast Control and Timing System (FCTS) which performs the trigger decision. Depending on the GLT output the FCTS can issue a 'Level 1 Accept' (L1A) or 'prescale' the trigger. A L1A results in the latency buffers for all sub-systems being read out for further processing. Prescaled triggers will only issue a L1A for for every $n$th event which pass their selection criteria. The priority of the L1T is to issue L1As for multihadron events which correspond to physics events of interest such as $B$ meson decays. Events such as Bhabha scattering used for luminosity calculations can be prescaled as this introduces no bias. The GLT contains both pure DCT and pure EMT L1As allowing unbiased efficiency measurements to be made for both of these trigger subsystems. The DCT and EMT are independently up to $99 \%$ efficient in identifying $B \bar{B}$ events, demonstrating the redundancy in the L1T. Output rates for L1As are around $2.5 \mathrm{kHz}$ under typical 
running conditions with the L1T contributing less than $1 \%$ of deadtime ${ }^{6}$ in the BABAR data acquisition system.

\subsubsection{The Software Trigger}

Events passing a L1A are processed in real time with an online computing farm and passed through the L3T. All event information is available to the L3T allowing a more sophisticated event selection process than the L1T, such as better track reconstruction and EMC energy clustering. Event filters select and classify events of interest using predefined scripts which contain selection criteria for different physics processes. The L3T reduces the output rate by around an order of magnitude compared to the L1T. Events passing the L3T filters are written to a temporary event store before being passed to offline computing farms which perform a more complete event reconstruction allowing for detailed quality monitoring before data are made available for analysis.

\footnotetext{
${ }^{6}$ Deadtime refers to periods when new data cannot be read out due to previous data being processed.
} 


\section{Chapter 3}

\section{Event Selection}

Collision events recorded by the BABAR detector are passed through a number of general and custom software filters in order to extract physics signatures of interest. This chapter describes the selection techniques used to maximise $B \rightarrow X_{s / d} \gamma$ [41] event extraction while efficiently rejecting combinatoric backgrounds. An overview of the analysis method is given and the variables used to distinguish between signal and background events are defined. Finally the development, optimisation and implementation of custom event filters are described.

\subsection{Analysis Overview}

Making a fully inclusive measurement of $B \rightarrow X_{s / d} \gamma$ decays can provide a significant challenge due to their rarity. The relative high energy of the photon provides a powerful

\begin{tabular}{|l|l|l|}
\hline Mode & $B \rightarrow X_{d} \gamma$ & $B \rightarrow X_{s} \gamma$ \\
\hline 1 & $B^{0} \rightarrow \pi^{+} \pi^{-} \gamma$ & $B^{0} \rightarrow K^{+} \pi^{-} \gamma$ \\
2 & $B^{+} \rightarrow \pi^{+} \pi^{0} \gamma$ & $B^{+} \rightarrow K^{+} \pi^{0} \gamma$ \\
3 & $B^{+} \rightarrow \pi^{+} \pi^{-} \pi^{+} \gamma$ & $B^{+} \rightarrow K^{+} \pi^{-} \pi^{+} \gamma$ \\
4 & $B^{0} \rightarrow \pi^{+} \pi^{-} \pi^{0} \gamma$ & $B^{0} \rightarrow K^{+} \pi^{-} \pi^{0} \gamma$ \\
5 & $B^{0} \rightarrow \pi^{+} \pi^{-} \pi^{+} \pi^{-} \gamma$ & $B^{0} \rightarrow K^{+} \pi^{-} \pi^{+} \pi^{-} \gamma$ \\
6 & $B^{+} \rightarrow \pi^{+} \pi^{-} \pi^{+} \pi^{0} \gamma$ & $B^{+} \rightarrow K^{+} \pi^{-} \pi^{+} \pi^{0} \gamma$ \\
7 & $B^{+} \rightarrow \pi^{+} \eta \gamma$ & $B^{+} \rightarrow K^{+} \eta \gamma$ \\
8 & $B^{+} \rightarrow \pi^{+} \pi^{0} \pi^{0} \gamma$ & $B^{+} \rightarrow K^{+} \pi^{0} \pi^{0} \gamma$ \\
9 & $B^{0} \rightarrow \pi^{+} \pi^{-} \pi^{0} \pi^{0} \gamma$ & $B^{0} \rightarrow K^{+} \pi^{-} \pi^{0} \pi^{0} \gamma$ \\
\hline
\end{tabular}

TABle 3.1: Reconstructed exclusive final states. The $B \rightarrow X_{s} \gamma$ final states are obtained by substituting a charged pion for a charged kaon in the $B \rightarrow X_{d} \gamma$ final states. All $\pi^{0} \rightarrow \gamma \gamma$ and all $\eta \rightarrow \gamma \gamma$. 
experimental signature which simplifies the task of identifying signal decays. Indeed for $B \rightarrow X_{s} \gamma$ it is sufficient to detect the high energy photon without reconstructing the $X_{s}$ hadronic state $[42,43]$. However, due to the nearly identical photon spectrum, such techniques are impractical when measuring $B \rightarrow X_{d} \gamma$ decays due to the dominance of the $X_{s}$ sample. Instead this analysis reconstructs a subset of nine exclusive modes whose contributions are to be added, therefore making a semi-inclusive measurement. Consequently model-dependent corrections for unreconstructed contributions must be made to extract the fully inclusive branching fraction $(\mathrm{BF})$ and hence $\left|V_{t d} / V_{t s}\right|$. Table 3.1 lists the exclusive modes reconstructed ${ }^{1}$ for $B \rightarrow X_{d} \gamma$ and $B \rightarrow X_{s} \gamma$ decays. The $X_{s}$ hadronic states are defined by substituting a charged pion for a charged kaon in the corresponding $X_{d}$ hadronic state with the kaon charge determined by the flavour of the parent $B$ meson. Identical cuts are used for both samples leading to many of the experimental systematic errors cancelling in the ratio of branching fractions. Due to the excess of expected events in the CKM favoured $X_{s}$ sample all cuts and filters are optimised to provide the most statistically significant measure of the $X_{d}$ sample. Final state $\pi^{0}$ and $\eta$ mesons are reconstructed exclusively from the decays $\pi^{0} \rightarrow \gamma \gamma$ and $\eta \rightarrow \gamma \gamma$ which corresponds to $98.8 \%(39.4 \%)$ of the $\pi^{0}(\eta)$ decay fraction [14].

The analysis is divided into two bins of reconstructed hadronic mass: a low mass bin, $0.5 \leq m_{X}<1.0 \mathrm{GeV} / c^{2}$, and a high mass bin, $m_{X} \geq 1.0 \mathrm{GeV} / c^{2}$. This division is motivated by the distinct characteristics expected for signal decays in each mass bin, as discussed in section 1.2.3.2. Consequently simulated signal events used for analysis must reflect this distinction. The low mass $X_{d}\left(X_{s}\right)$ region is dominated by the resonant transition $B \rightarrow \rho / \omega \gamma\left(B \rightarrow K^{*} \gamma\right)$ and is therefore modelled exclusively with these decays. Resonant particle masses are modelled as Breit-Wigner distributions using the world average width for that particle [14]. The high mass bin is modelled as a cocktail of non-resonant decays according to the $\mathrm{KN}$ photon spectrum [27] using $m_{X}^{T}=1.0 \mathrm{GeV} / c^{2}$. The hadronic state is generated through phase space decays from JETSET [44]. This model does not account for the vector resonance contributions with masses greater than $1.0 \mathrm{GeV} / c^{2}$ such as high end tails of the $K^{*}$ and $\rho$ distributions or the measured $B \rightarrow$ $K_{2}^{*}(1430) \gamma$ transition [14]. It is assumed that the low mass resonance tails are to a good approximation accounted for in the JETSET phase space distribution; the variation of hadronic final states due to higher mass resonances is considered as a source of systematic uncertainty in section 5.2.2. The upper limit of the high mass bin is chosen such that the non-resonant measurement be as inclusive as possible while considering the increase in combinatoric backgrounds at higher values of $m_{X}$. This is primarily constrained by computational limitations implementing the pre-reconstruction event filters described in

\footnotetext{
${ }^{1}$ The choice of reconstructed modes is based on those used in the previous version of this analysis: modes 1-7 [1]. Modes 8 and 9 were added here with the aim of making a more inclusive measurement; however, their addition was found to be a disadvantage in the statistical optimisation discussed below.
} 
section 3.4. Post-reconstruction the choice of upper mass limit forms part of the cut optimisation procedure using simulated data, discussed in section 3.6.7.

Overall there are four experimental measurements: the semi-inclusive BF obtained from the sum of exclusive reconstructed states in the low and high mass bins for $B \rightarrow X_{d} \gamma$ and $B \rightarrow X_{s} \gamma$ transitions. The signal yields of these transitions are extracted from experimental data using a two dimensional extended maximum likelihood fit which is described in chapter 4 . The measured BFs are then used to estimate total BFs in each mass region using a model dependent extrapolation. The extrapolated BFs are combined for each flavour to give fully inclusive BFs, the ratio of which are then used to extract a value for $\left|V_{t d} / V_{t s}\right|$ according to the theoretical relations discussed in chapter 1.

\subsubsection{Simulated and Experimental Data Samples}

This analysis uses $429.1(44.8) \mathrm{fb}^{-1}$ of experimental data recorded with CM energy at $(\sim 40 \mathrm{MeV}$ below) the $\Upsilon(4 S)$ resonance. Data recorded at the $\Upsilon(4 S)$ corresponds to $(470.9 \pm 2.8) \times 10^{6} B \bar{B}$ pairs [45]. The analysis is optimised using Monte Carlo (MC) simulated data and then applied to the experimental data, thus eliminating bias which can arise from basing the event selection and distribution modelling on real data. The $\mathrm{MC}$ data used in this analysis at least match the size of the experimental dataset. In the $B A B A R$ simulation framework particle decays are generated from a database of allowed decay modes, each with a given $\mathrm{BF}$, and the daughter particle kinematics are determined from a phase space model of the decay. Responses of the detector subsystems are modelled using the GEANT4 package [46] and QED radiative corrections are modelled with PHOTOS [47].

To optimise custom event filters for $B \rightarrow X_{d} \gamma$ signal extraction, simulations of both signal events and events which can contribute to combinatoric backgrounds are required. Table 3.2 lists the different simulated datasets used for analysis optimisation, showing the total number of generated events. The first nine $\mathrm{MC}$ datasets in table 3.2 are signal decays $^{2}$ whereby one generated $B$ meson is forced to decay to the final state indicated and the other can decay to any allowed final state. Signal decays do not directly correspond to the reconstructed final states listed in table 3.1; they contain all allowed final states for the modelled transition, i.e. all allowed decays of the resonant particle in the low mass region or all allowed phase space decays from JETSET in the high mass region. The reconstructed decays therefore form a subset of the signal MC data. Due to the

\footnotetext{
${ }^{2}$ Note that MC data modelling the high mass bin are generated separately for charged and neutral $B$ meson decays. To distinguish these the hadronic component of the decay is labelled with a double subscript, the first component indicating the penguin transition quark flavour and the second component indicating the flavour of the $B$ meson spectator quark.
} 


\begin{tabular}{|l|l|l|}
\hline Event Class & Simulated Transition & Simulated Events \\
\hline \hline KN and JETSET Signal & $B^{+} \rightarrow X_{d u} \gamma$ & $1,288,000$ \\
& $B^{0} \rightarrow X_{d d} \gamma$ & $1,288,000$ \\
& $B^{+} \rightarrow X_{s u} \gamma$ & $11,178,000$ \\
& $B^{0} \rightarrow X_{s d} \gamma$ & $11,178,000$ \\
\hline Vector Resonance Signal & $B^{+} \rightarrow \rho^{+} \gamma$ & 650,000 \\
& $B^{0} \rightarrow \rho^{0} \gamma$ & 650,000 \\
& $B^{0} \rightarrow \omega \gamma$ & 650,000 \\
& $B^{+} \rightarrow K^{*+} \gamma$ & $6,449,000$ \\
& $B^{0} \rightarrow K^{* 0} \gamma$ & $6,449,000$ \\
\hline Generic Background & $B^{+} B^{-}$ & $708,762,000$ \\
& $B^{0} \bar{B}^{0}$ & $717,995,000$ \\
& $c \bar{c}$ & $1,128,544,000$ \\
& $q \bar{q}(q=u, d, s)$ & $1,662,404,000$ \\
\hline
\end{tabular}

TABLE 3.2: Simulated datasets used for event selection optimisation showing the number of generated events before any cuts are applied.

\begin{tabular}{|l|l|l|}
\hline Simulated Transition & Events $\geq m_{X}^{T}$ & Fraction \\
\hline$B^{+} \rightarrow X_{d u} \gamma$ & $1,201,936$ & 0.933 \\
$B^{0} \rightarrow X_{d d} \gamma$ & $1,201,708$ & 0.933 \\
$B^{+} \rightarrow X_{s u} \gamma$ & $10,425,400$ & 0.933 \\
$B^{0} \rightarrow X_{s d} \gamma$ & $10,429,063$ & 0.933 \\
\hline
\end{tabular}

TABLE 3.3: High mass bin simulated signal sizes after model based hadronic mass cut. The fraction of events remaining after the cut, relative to the full generated sample is also shown.

\begin{tabular}{|l|l|l|}
\hline Simulated Transition & Events $<m_{X}^{T}$ & Fraction \\
\hline$B^{+} \rightarrow \rho^{+} \gamma$ & 568,518 & 0.875 \\
$B^{0} \rightarrow \rho^{0} \gamma$ & 569,089 & 0.876 \\
$B^{0} \rightarrow \omega \gamma$ & 650,000 & 1.000 \\
$B^{+} \rightarrow K^{*+} \gamma$ & $5,752,171$ & 0.892 \\
$B^{0} \rightarrow K^{* 0} \gamma$ & $5,727,473$ & 0.888 \\
\hline
\end{tabular}

TABLE 3.4: Low mass bin simulated signal sizes after model based hadronic mass cut. The fraction of events remaining after the cut, relative to the full generated sample is also shown. 
independent signal modelling in each mass bin, the boundary between signal samples is identified by placing the threshold cut of $m_{X}^{T}=1.0 \mathrm{GeV} / c^{2}$ on the generated hadronic mass. The number of simulated events for each sample after this cut are given in tables 3.3 and 3.4 for the high and low mass bins respectively. Normalisation of signal MC data to the $B A B A R$ recorded luminosity assume total inclusive BFs of $1 \times 10^{-5}\left(3.6 \times 10^{-4}\right)$ for $B \rightarrow X_{d} \gamma\left(B \rightarrow X_{s} \gamma\right)$ decays. These BFs are taken from the expected order of magnitude of theoretical estimates in the case of $B \rightarrow X_{d} \gamma$ [22] and the world experimental average in the case of $B \rightarrow X_{s} \gamma$ [14]. The relative contributions of each vector resonance in the respective low mass bins are taken from world average BFs [14]. These resonant BFs are corrected to account for the fraction of events expected below $m_{X}^{T}$ using the MC data fractions shown in table 3.4. For $B \rightarrow \rho / \omega \gamma\left(B \rightarrow K^{*} \gamma\right)$ transitions the calculated contribution corresponds to $10.2 \%(10.5 \%)$ of the above assumed inclusive widths.

Generic $B$ pair events simulate other $\Upsilon(4 S) \rightarrow B \bar{B}$ processes with both $B$ mesons decaying to SM final states which are determined from a database of $B \bar{B}$ decays. The database essentially lists the BFs of known $B$ meson decays and uses JETSET to generate higher multiplicity modes which have generally not been measured. These data include $B \rightarrow X_{s} \gamma$ events (but not $B \rightarrow X_{d} \gamma$ events) which must be vetoed to avoid double counting. Any signal or generic event with the decay of a neutral $B$ meson pair models the effect of neutral meson mixing during event generation. Generic continuum events of light quark pairs from $e^{+} e^{-} \rightarrow q \bar{q}(q=u, d, s, c)$ transitions use JETSET [44] to simulate the hadronisation of the quark pair. Events from $e^{+} e^{-} \rightarrow l^{+} l^{-}(l=e, \mu, \tau)$ processes are found to contribute at a negligible level to combinatoric backgrounds and hence are ignored in MC data studies.

The simulated events produced for BABAR analyses are known not to model the data perfectly. To improve the agreement for neutral particles reconstructed with data from the EMC, asymmetric energy smearing and edge effect corrections are made [48]. This reduces systematic errors arising from differences between experimental data and MC events for such particles and is particularly important for $B \rightarrow X_{s / d} \gamma$ events where the photon deposits a large fraction of the total event energy in the EMC.

\subsection{Event Reconstruction Framework}

Data recorded by the BABAR detector which pass quality control checks are made available for analysis. Both experimental and MC data are regularly reprocessed to take advantage of improved central software filters. Each reprocessing cycle has a unique accessible dataset. Reconstruction software is interfaced through a custom designed central $\mathrm{C}++$ analysis framework consisting of a number of software packages each dedicated to 
to a subset of the reconstruction and which are called by the analyst as required. Collectively the packages are maintained and updated within numbered software releases. The analysis presented here uses reprocessed datasets labelled R24a with BABAR release number 24.3.6, the most recent stable release for event reconstruction at the time of analysis. Detector conditions for a given running period and lookup tables for efficiency corrections in MC data are accessed from dedicated conditions databases. This analysis uses the conditions database cond24boot09 which is the recommended database for the datasets and release number used.

Lists of fundamental reconstructed particles such as charged tracks and neutral clusters in the EMC are contained in a central event store for both real data and those simulated with GEANT4. All composite particles are reconstructed from these fundamental lists. Common composite particles, such as $\pi^{0}$ and $\eta$ mesons, are reconstructed within the common analysis framework and are available at runtime. The following provides a summary of the lists used for reconstruction in this analysis. All cuts are made in the laboratory rest frame unless otherwise stated.

\subsubsection{Charged Tracks}

Charged tracks are taken from the list GoodTracksLoose which requires a momentum magnitude $\leq 10 \mathrm{GeV} / c$ and transverse momentum $\geq 0.05 \mathrm{GeV} / c$. Additionally these tracks must have a distance of closest approach to the IR of $|z|<2.5 \mathrm{~cm}$ along the principle axis and $<1.5 \mathrm{~cm}$ in the transverse plane. All tracks have an assumed pion mass hypothesis.

\subsubsection{Photons}

Photons are taken from the list GoodPhotonLoose. These consist of single bump EMC clusters without an associated charged track which have an energy deposit of $\geq 0.03 \mathrm{GeV}$ and lateral moment $\leq 0.8$. A photon mass hypothesis is applied.

\subsection{3 $\pi^{0}$ and $\eta$ Mesons}

Composite neutral meson candidates from dual photon decays are obtained from the lists piODefaultMass and etaggDefault for $\pi^{0}$ and $\eta$ mesons respectively. These lists are constructed by adding the four-momentum of photon pairs from the GoodPhotonLoose list, with the additional requirement that photon energy deposits satisfy $0.03 \leq E_{\gamma} \leq$ $10.0\left(0.05 \leq E_{\gamma} \leq 10.0\right) \mathrm{GeV}$ and the invariant mass of photon pairs are in the range 
$0.115 \leq m_{\gamma \gamma} \leq 0.150\left(0.470 \leq m_{\gamma \gamma} \leq 0.620\right) \mathrm{GeV} / c^{2}$ for $\pi^{0}(\eta)$ meson candidates. Additionally $\eta$ meson candidates are required to have a momentum magnitude in the range $0.2 \leq\left|p_{\eta}\right| \leq 10.0 \mathrm{GeV} / c$ and $\pi^{0}$ candidates must have energy $\geq 0.2 \mathrm{GeV}$. After satisfying these criteria the neutral candidates are refitted constraining the particle origin to the primary vertex (defined below) and their masses to the world average value [14]. This gives an unbiased improvement on the kinematic resolution of the particle which is limited due to detector resolution prior to refitting. Higher kinematic resolution is desirable when using the reconstructed neutrals to form composite candidates higher up the decay chain.

\subsubsection{Vertex Fitting}

Where composite candidates are reconstructed from two or more charged tracks fitting algorithms can determine the spatial coordinates of the origin of those tracks: the decay vertex of the composite particle. This analysis uses the BABAR fitting routine Cascade [49] whose goal is iterative $\chi^{2}$ minimisation on the vertex hypothesis of the input particles. The Cascade fitter implicitly requires that momentum is conserved at a decay vertex, although particle momenta are allowed to vary within their measured errors in the fit. An additional geometric constraint requiring that all included tracks originate from the same spatial point can also be applied. Neutral particles, which are assumed to originate from the primary vertex, will have their momentum redefined so that they originate from the common decay vertex if they are included in the fit.

\subsubsection{Charged Particle Identification Classifiers}

As discussed in chapter 2 a number of variables, such as $d E / d x$ in the tracking subsystems and Cherenkov angle in the DIRC, can be employed for the purpose of charged track PID. The usefulness of individual variables varies substantially for a given track flavour and momentum. To simplify the task of charged track PID BABAR employs a dedicated PID group who combine such variables into multivariate classifiers trained on well defined control samples. Generally the classifiers are accessed through PID selectors which give a binary output depending on whether the classifier output has passed a predefined cut. A single classifier can have multiple selectors depending on the tightness of cut on the classifier output, typically these are labelled VeryLoose, Loose, Tight, VeryTight and SuperTight. For each selector the PID group carry out momentum dependent efficiency and misidentification rate studies on real and simulated data. This analysis is primarily concerned with the discrimination between charged pions and kaons in order to separate $B \rightarrow X_{s} \gamma$ and $B \rightarrow X_{d} \gamma$ decays. For pion identification a multi-class 
learning classifier, pionKM, is used [50]. This classifier is trained with 36 input variables from the SVT, DCH, DIRC and EMC using control samples of $D^{*+} \rightarrow D^{0}\left(K^{-} \pi^{+}\right) \pi^{+}$, $K_{S} \rightarrow \pi^{+} \pi^{-}$and $\tau \rightarrow \nu_{\tau} 3 \pi$ decays. For kaon identification a bagged decision tree classifier, kaonBDT, is used [50]. The classifier is trained on $D^{*+} \rightarrow D^{0}\left(K^{-} \pi^{+}\right) \pi^{+}$control samples again with 36 input variables. The kaon classifier has an additional selector representing a looser cut than VeryLoose; this is labelled NotAPion. Control sample studies by the PID group show that the typical rate of charged kaons being misidentified as pions is less than $10 \%$ for all momenta.

\subsubsection{Primary Vertex}

The primary interaction in a $e^{+} e^{-}$collision event occurs at the PEP-II IR. The spatial position of this interaction can be determined from measurement of the beamspot. The size of the beamspot is typically 150 microns in $x, 6$ microns in $y$ and $1 \mathrm{~cm}$ in $z$. For event reconstruction purposes a more accurate determination, particularly of the $z$ coordinate, is required. This is achieved in the BABAR framework by fitting for the primary vertex on an event by event basis [49]. The fit combines all tracks with impact parameter in the transverse plane of less than $1 \mathrm{~mm}$ from the beamspot centre. For $B \bar{B}$ events the primary vertex will be shifted in the positive $z$ direction from the $e^{+} e^{-}$interaction point due to the average $B$ meson lifetime [14]. Essentially the primary vertex represents the mid-point between the two $B$ meson decays. This is illustrated schematically in figure 3.1. In general neutral particle candidates within the BABAR framework have their fourmomentum defined such that they originate from the primary vertex by default. The primary vertex resolution in a $B \bar{B}$ event is typically 100 microns in $x$ and 115 microns in $z$. The $y$ resolution from the primary vertex fit is around a factor of ten worse than the existing beamspot measurement, hence the beamspot measurement is used to constrain this coordinate.

\subsection{Discriminating Variables}

There are many variables which aid in the discrimination of reconstructed candidates from signal decay events compared to those originating from combinatoric backgrounds. These can broadly be separated into kinematic, topological and so-called 'tagging' variables. The variables considered in this analysis are defined here. 


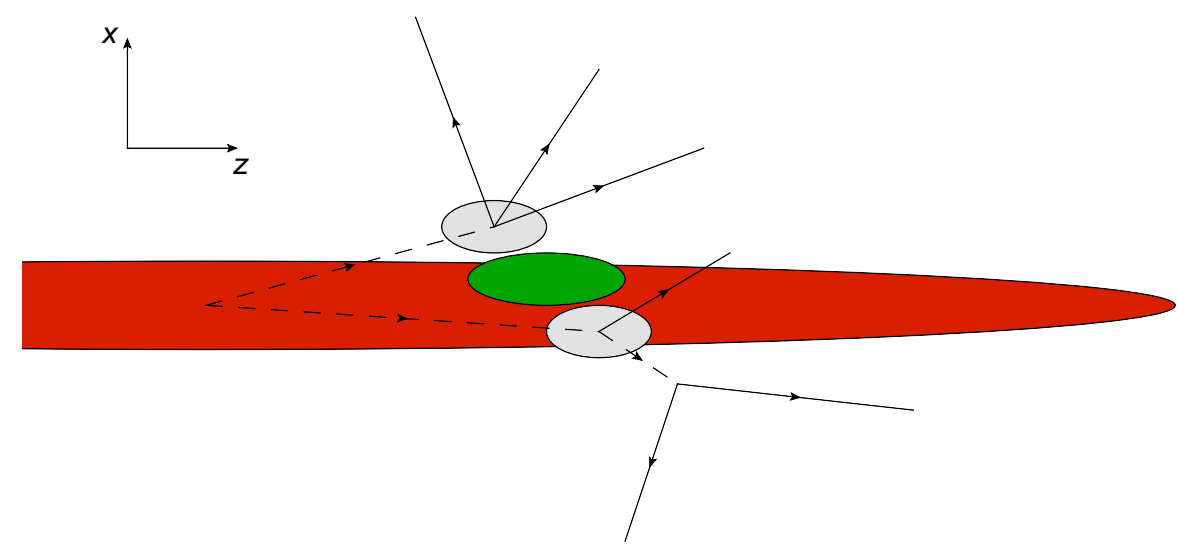

Figure 3.1: Schematic representation of the primary vertex for a $B \bar{B}$ event in the $x-z$ plane. The red ellipse represents the beamspot resolution. The green ellipse indicates the improved primary vertex resolution, particularly in the $z$ direction. The grey ellipses show the respective $B$ meson decay vertex resolutions. Note that the primary vertex is distinct from the $\Upsilon(4 S)$ decay vertex. The resolutions shown are not to scale.

\subsubsection{Kinematic Variables}

In addition to placing kinematic cuts on the energy and momentum of reconstructed particles there are two kinematic variables of primary importance when considering $B$ meson decays at BABAR. These are the beam energy substituted mass, $m_{E S}$, and the difference between the reconstructed and expected energy of the $B$ meson, $\Delta E$ [51]. Explicitly they are defined as,

$$
\begin{aligned}
m_{E S} & =\sqrt{\frac{1}{4} s-\left|\mathbf{p}_{B}^{*}\right|^{2}}, \\
\Delta E & =E_{B}^{*}-\frac{1}{2} \sqrt{s}
\end{aligned}
$$

where $\left(E_{B}^{*}, \mathbf{p}_{B}^{*}\right)$ is the four-momentum of the reconstructed $B$ meson and $s$ is the Mandelstam variable for the transition $e^{+} e^{-} \rightarrow \Upsilon(4 S)$, i.e. the CM energy squared. The asterisk superscript denotes a quantity in the $\mathrm{CM}$ frame. Both variables make use of the fact that the $\Upsilon(4 S)$ resonance lies just above the $B \bar{B}$ threshold which results in each $B$ meson having a CM energy half that of $\sqrt{s}$. It follows that $\Delta E$ will peak at zero for a correctly reconstructed $B$ meson with an approximate Gaussian distribution resulting from detector resolution limits. The negative tail will be more pronounced due to energy leakage in the EMC which for a radiative decay such as the signal considered can be significant. The beam energy substituted mass is preferential to the reconstructed $B$ mass as the beam energy is measured to a high degree of accuracy and does not suffer from detector resolution limitations inherent in the measurement of $E_{B}^{*}$. Furthermore an alternative mass hypothesis for charged tracks will not change the value of $m_{E S}$ 
whereas a correct measurement of $E_{B}^{*}$ depends on the flavour of all tracks being correctly identified. For a correctly reconstructed $B$ decay, $m_{E S}$ will peak at the $B$ mass, $m_{B}=5.279 \mathrm{GeV} / c^{2}$, with a Gaussian shape due to detector momentum resolution and the resolution in beam energy measurement. On average $m_{E S}$ is observed to have an enhanced negative tail due to EMC energy leakage in the detection of neutral particles. To minimise the effect of energy leakage from the signal photon it is useful to define $m_{E S}^{\prime}$, whereby the magnitude of $\mathbf{p}_{B}^{*}$ is recalculated constraining the photon energy such that $\Delta E=0$, i.e.

$$
E_{\gamma}^{*}+E_{X}^{*}-\frac{1}{2} \sqrt{s}=0
$$

Here $E_{\gamma}$ is the photon energy and $E_{X}$ is the energy of the hadronic component of the decay. The potential introduction of a correlation between $\Delta E$ and $m_{E S}^{\prime}$ is discussed in section 4.1.3. Figures 3.2 and 3.3 show respectively simulated distributions for $m_{E S}^{\prime}$ (including an overlay of the corresponding signal $m_{E S}$ distribution ${ }^{3}$ ) and $\Delta E$ for correctly reconstructed candidates and continuum background before any post-reconstruction cuts. The signal resolution is $\sim 50 \mathrm{MeV}$ for $\Delta E$ and $\sim 5 \mathrm{MeV} / c^{2}$ for $m_{E S}^{\prime}$. The negative tail due to energy leakage can be seen in the signal distributions of both variables.

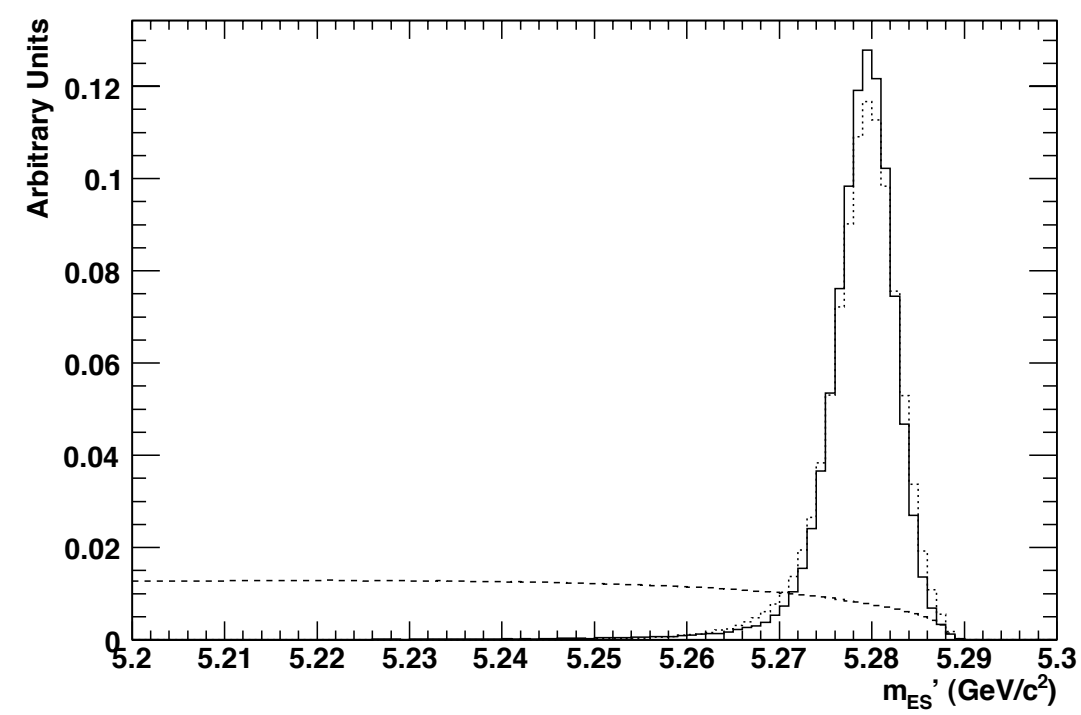

Figure 3.2: Normalised distributions of $m_{E S}^{\prime}$ for continuum (dashed line) and correctly reconstructed candidates (solid line) before post-reconstruction cuts. The dotted line overlays the distribution of $m_{E S}$ for correctly reconstructed candidates to illustrate the resolution improvement obtained using $m_{E S}^{\prime}$.

\footnotetext{
${ }^{3}$ Note that the improvement in resolution of $m_{E S}^{\prime}$ over $m_{E S}$ is relatively minor. This is in contrast to early radiative penguin analyses at $B A B A R$ due to an improved energy leakage correction during EMC cluster reconstruction. However, to be consistent with earlier analyses $m_{E S}^{\prime}$ is generally preferred over $m_{E S}$ as a discriminating variable.
} 


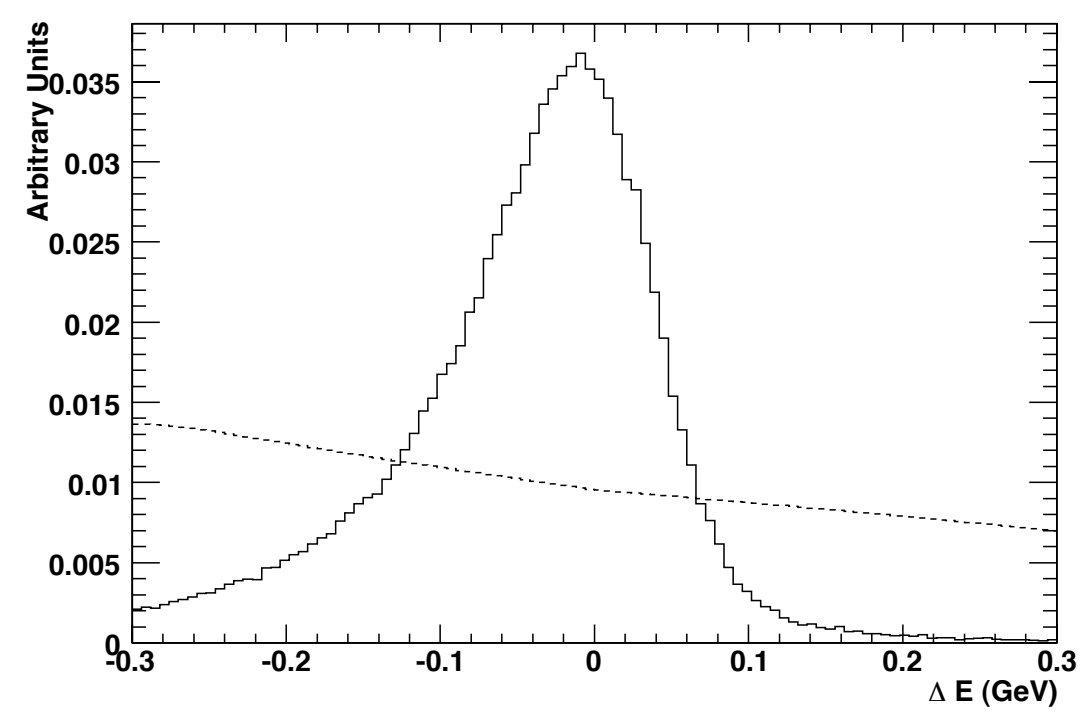

FiguRE 3.3: Normalised distributions of $\Delta E$ for continuum (dashed line) and correctly reconstructed candidates (solid line) before post-reconstruction cuts.

\subsubsection{Topological Variables}

Measuring the topology of detected particles in a given event can provide powerful discrimination between events arising from $\Upsilon(4 S)$ decays and those arising from continuum backgrounds. The $B$ mesons are produced almost at rest in the $\Upsilon(4 S)$ frame and hence decay isotropically in that frame. Conversely particles produced in continuum events typically have large kinetic energies in the CM frame and form collimated jets along the axes of the initial quark and anti-quark pair. Therefore variables which distinguish the isotropic or jet-like nature of events can aid in the reduction of continuum backgrounds.

Continuum events containing reconstructed $B \rightarrow X_{s / d} \gamma$ candidates will have an associated high energy photon. Where this photon is a daughter of the decay chain from the initial quark hadronisation its direction will be highly correlated with other particles in the corresponding jet. Continuum background also arises from interactions involving initial state radiation (ISR), $e^{+} e^{-} \rightarrow q \bar{q} \gamma$. In this case the photon momentum will be largely independent of the individual jet directions. However, together both jets will be seen to recoil against the photon due to momentum conservation. It follows that the measurement of event shape variables with respect to the high energy photon can be used to further reduce continuum backgrounds.

In order to calculate many of the variables which can separate these event topologies it is necessary to distinguish between charged tracks and neutral clusters which were used to reconstruct the signal candidate and those which form the rest of the event 
(ROE). For correctly reconstructed candidates the ROE is assumed to contain all particles detected from the other $B$ meson decay in the event. All topological variables are calculated in the CM frame unless otherwise stated. In the following figures MC data distributions for correctly reconstructed candidates and continuum backgrounds before post-reconstruction cuts are presented.

\subsubsection{Thrust}

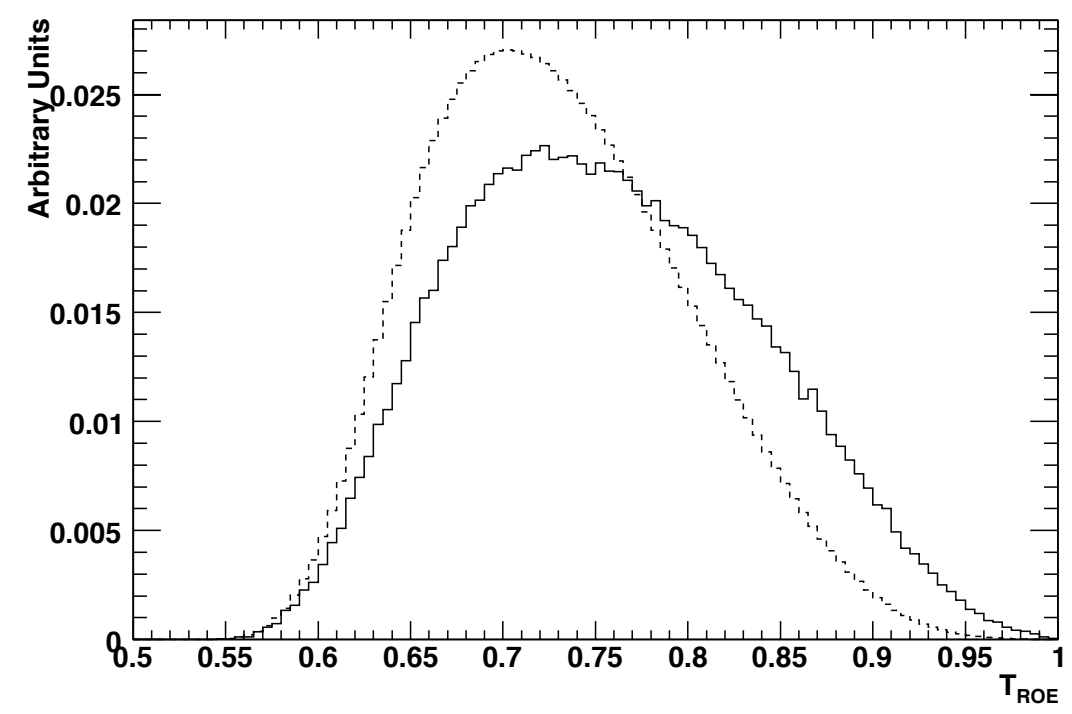

Figure 3.4: Normalised distributions of $T_{R O E}$ for continuum (dashed line) and correctly reconstructed candidates (solid line) before post-reconstruction cuts.

The thrust of $N$ detected particles each with momentum $\mathbf{p}_{i}$ is defined as,

$$
T=\frac{\sum_{i}^{N}\left|\mathbf{n} \cdot \mathbf{p}_{i}\right|}{\sum_{i}^{N}\left|\mathbf{p}_{i}\right|},
$$

where $\mathbf{n}$ is the unit vector which maximises the value of $T$ and whose direction defines the thrust axis of those particles [52]. Figure 3.4 shows the calculated thrust for the ROE, $T_{R O E}$. It can be seen that on average continuum events have a lower thrust for the ROE than correctly reconstructed signal events as they are less isotropic.

Another useful variable derived from the calculated thrust is the cosine of the angle between the photon momentum and the thrust axis of the ROE, $\left|\cos \theta_{T}\right|$. This has a uniform distribution for correctly reconstructed signal events whereas jet-like continuum events peak at unity. This difference is illustrated in figure 3.5. 


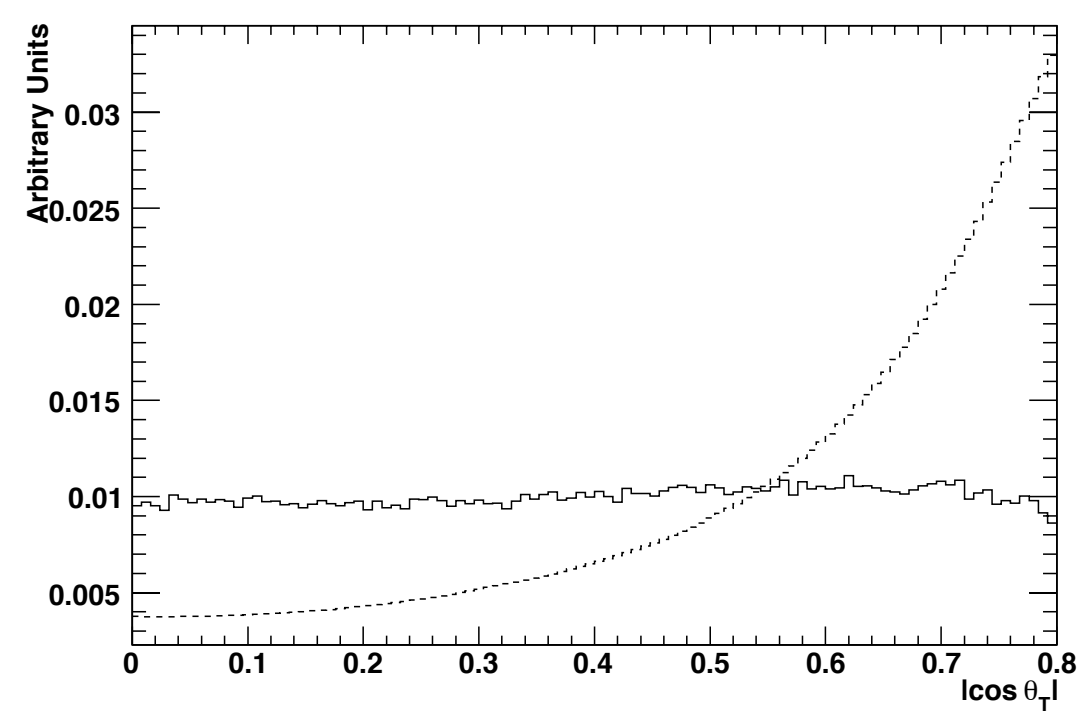

Figure 3.5: Normalised distributions of $\left|\cos \theta_{T}\right|$ for continuum (dashed line) and correctly reconstructed candidates (solid line) before post-reconstruction cuts.

\subsubsection{Energy and Momentum Flow}

A number of useful topological variables measure the angular distribution of energy and momentum flow in a given event. This analysis makes use of Fox-Wolfram moments [53] and both longitudinal and perpendicular 'monomial' functions [54].

For $N$ particles with total energy $E_{\text {all }}$, the $l$ th normalised Fox-Wolfram moment is defined as,

$$
H_{l}=\sum_{i, j}^{N} \frac{\left|\mathbf{p}_{i}\right|\left|\mathbf{p}_{j}\right|}{E_{\text {all }}} \mathcal{P}_{l}\left(\cos \theta_{i j}\right),
$$

where $\mathcal{P}_{l}$ are Legendre polynomials of order $l$ and $\theta_{i j}$ is the angle subtended between the momentum vectors of particles $i$ and $j$. The ratio of the second to zeroth Fox-Wolfram moments, $R_{2}=H_{2} / H_{0}$, is commonly used to measure the jet-like nature of an event. To reduce ISR backgrounds $R_{2}$ is calculated in the frame recoiling against the high energy photon for all particles excluding the photon. To distinguish this frame from the CM frame a prime notation is used, $R_{2}^{\prime}$. Figure 3.6 shows the simulated distributions of $R_{2}^{\prime}$. It can be seen that the signal distribution peaks closer to zero relative to the continuum background.

The normalised monomial functions are calculated relative to a given axis, $\alpha$. For $N$ particles whose momenta $\mathbf{p}_{i}$ subtend an angle $\theta_{i}$ with respect to $\alpha$ the $l$ th monomial is 


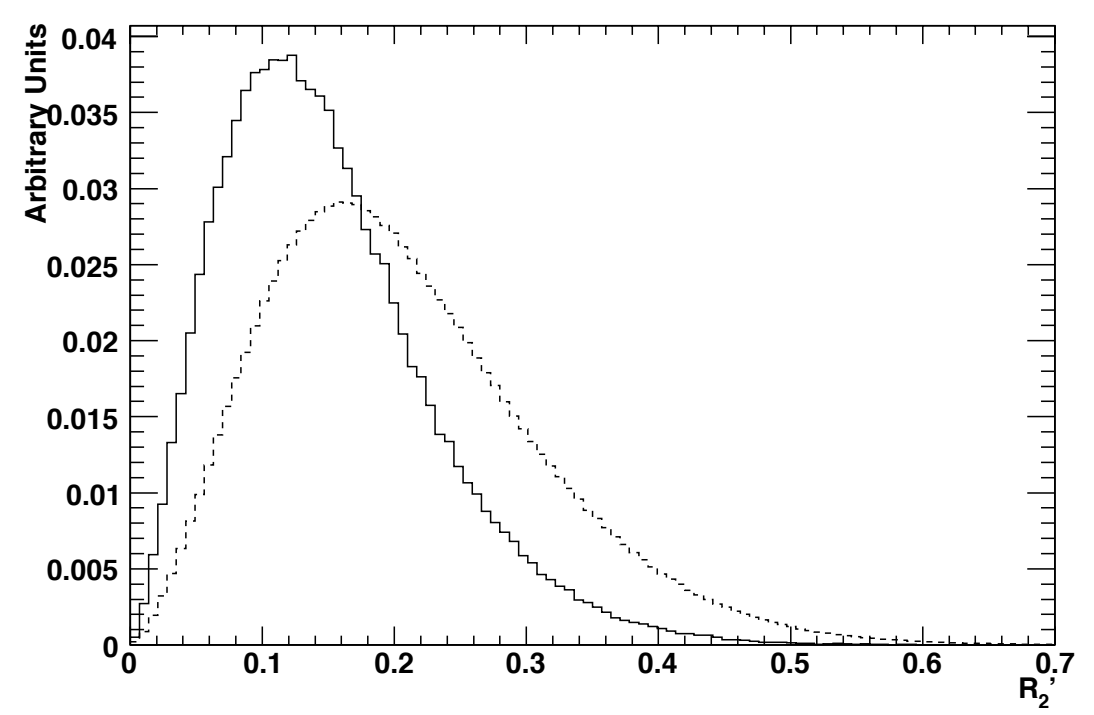

FIGURE 3.6: Normalised distributions of $R_{2}^{\prime}$ for continuum (dashed line) and correctly reconstructed candidates (solid line) before post-reconstruction cuts.

defined as,

$$
\begin{aligned}
L_{l}^{\alpha} & =\frac{\sum_{i}^{N}\left|\mathbf{p}_{i}\right| \cos ^{l} \theta_{i}}{\sum_{i}^{N}\left|\mathbf{p}_{i}\right|}, \\
P_{l}^{\alpha} & =\frac{\sum_{i}^{N}\left|\mathbf{p}_{i}\right| \sin ^{l} \theta_{i}}{\sum_{i}^{N}\left|\mathbf{p}_{i}\right|}
\end{aligned}
$$

for the longitudinal and perpendicular monomials respectively. This analysis considers the monomials functions of the $\mathrm{ROE}$ with respect to the thrust axis of the $\mathrm{ROE}$ and with respect to the high energy photon momentum axis. Figures comparing distributions for correctly reconstructed candidates and continuum backgrounds for each of the twelve monomials are contained in appendix A.

\subsubsection{Sphericity Tensor}

The sphericity tensor of an event is defined as,

$$
S^{\alpha \beta}=\frac{\sum_{i}^{N} p_{i}^{\alpha} p_{i}^{\beta}}{\sum_{i}^{N}\left|\mathbf{p}_{i}\right|^{2}},
$$

where the indices $\alpha, \beta=1,2,3$ denote the $x, y$ and $z$ three momentum components of the $i$ th particle [55]. The resulting $3 \times 3$ matrix can be diagonalised giving three eigenvalues, $\lambda_{1} \geq \lambda_{2} \geq \lambda_{3}$ with $\lambda_{1}+\lambda_{2}+\lambda_{3}=1$. From these eigenvalues the sphericity, $S$, planarity, 
$P$ and aplanarity, $A$, for $N$ particles are defined respectively as,

$$
\begin{aligned}
S & =\frac{3}{2}\left(\lambda_{1}+\lambda_{2}\right), \\
P & =\lambda_{2}-\lambda_{1}, \\
A & =\frac{3}{2} \lambda_{1} .
\end{aligned}
$$

Each of these variables has a distinct distribution for isotropic and jet-like events due to differences in the typical sphericity tensor for each topology. Figures showing the distributions of each sphericity variable for the ROE, comparing continuum and correctly reconstructed candidates, are contained in appendix A.

\subsubsection{Angular Momentum}

A final topological variable presents itself from angular momentum, the cosine of the angle between the signal candidate $B$ meson momentum and the principal axis, $\left|\cos \theta_{B}\right|$. In a signal event the spin-1 $\Upsilon(4 S)$ decays to two spin-0 $B$ mesons hence the angular distributions of $B$ mesons is proportional to $\sin ^{2} \theta_{B}$, while fake $B$ meson candidates from continuum decays are generally observed to have uniform distribution in $\left|\cos \theta_{B}\right|$. This distinction can be seen in figure 3.7 which shows simulated distributions of $\left|\cos \theta_{B}\right|$.

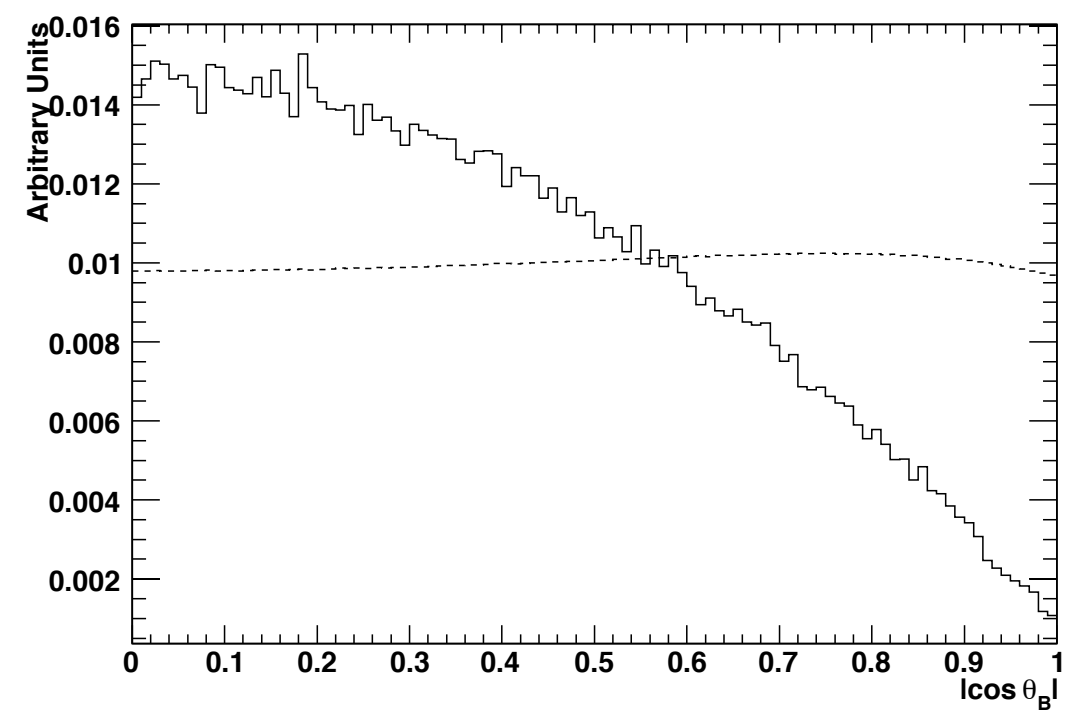

Figure 3.7: Normalised distributions of $\left|\cos \theta_{B}\right|$ for continuum (dashed line) and correctly reconstructed candidates (solid line) before post-reconstruction cuts. 


\subsubsection{Flavour Tagging Variables}

Many analyses of $B$ meson decays in $B A B A R$ data require the flavour of both $B$ mesons to be known from their respective decays. Typically one $B$ meson is fully reconstructed and knowledge of the flavour of the other $B$ meson in an event is required. The properties of this particle, the so-called 'tag $B$ ', can be inferred from the ROE and a variety of tools exist in the BABAR framework to carry out this task. Although the analysis presented here does not explicitly depend on knowledge of the $\operatorname{tag} B$, its properties can aid in the rejection of combinatoric backgrounds from continuum events.

Variables used to determine the flavour of the $\operatorname{tag} B$ meson aim to establish the $b$ quark flavour by identifying correlations between the quark charge and the signed characteristics of the decay products [56]. The dedicated BABAR tagging group combine such variables into multivariate classifiers, known as sub-taggers, with different sub-taggers corresponding to different physics processes. The sub-taggers are subsequently combined to assign a probability to the flavour of the $\operatorname{tag} B$ meson. Importantly for this analysis, the sub-taggers have distinct distributions for events containing $B$ meson decays compared to continuum events. Their outputs are thus useful discriminating variables. The complete tagging framework is described in detail in [57], an overview of seven sub-taggers used for this analysis is given here.

\subsubsection{Lepton Tagging}

Three of the sub-taggers rely on the lepton content of $B$ meson decays. In $b \rightarrow c$ transitions a direct semi-leptonic decay will result in a lepton with the same sign charge as the parent $b$ quark. Alternatively a lepton from a cascade $b \rightarrow c \rightarrow s$ decay can have either the same or opposite sign charge; however, it will exhibit a softer momentum spectrum. It is therefore possible to use the charge and kinematics of leptons from the ROE to identify the $b$ quark flavour of the $\operatorname{tag} B$ meson. On average continuum events contain significantly fewer leptons, it follows that a classifier used to identify the lepton content of an event will have different performance on such data compared to signal $B$ meson decay events.

An electron sub-tagger, $L_{T A G}^{e}$, has been trained to identify events where PID selectors identify any track in the ROE to be an electron or positron. The classifier is a neural net with input variables that give kinematic information of the assumed semi-leptonic decay. These variables, all calculated in the CM frame, are the momentum of the electron track candidate, the energy in the hemisphere defined by the direction of the virtual $W$ boson in the assumed semi-leptonic decay and the cosine of the angle between the assumed 


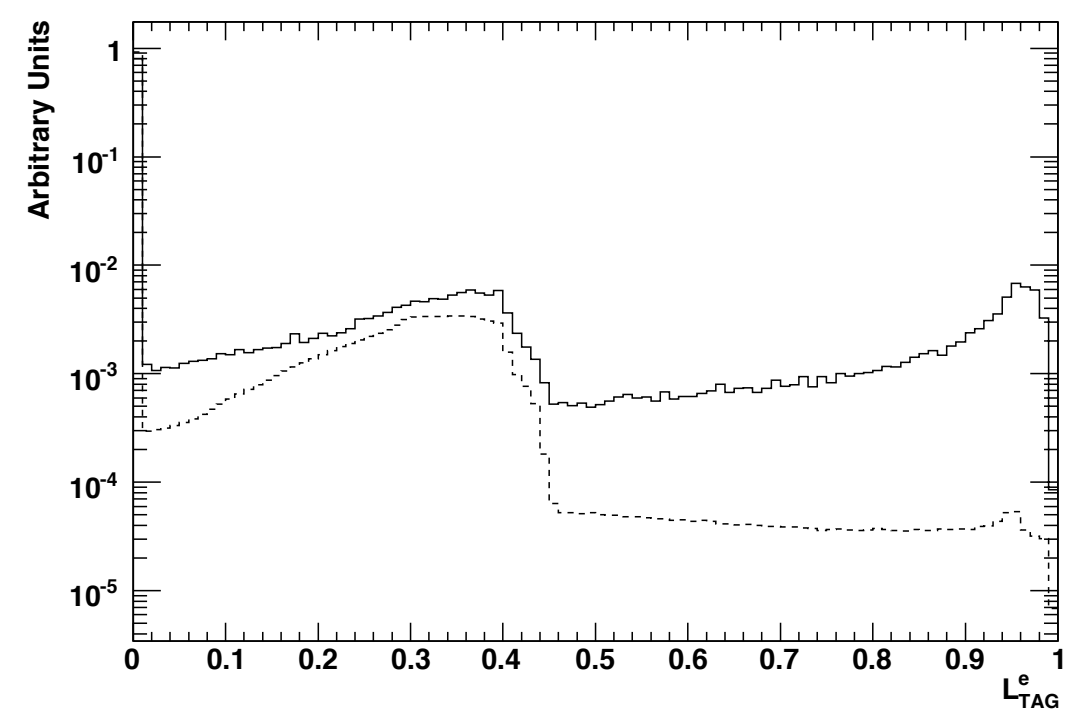

FiguRE 3.8: Normalised distributions of $L_{T A G}^{e}$ for continuum (dashed line) and correctly reconstructed candidates (solid line) before post-reconstruction cuts. Note the $\log$ scale.

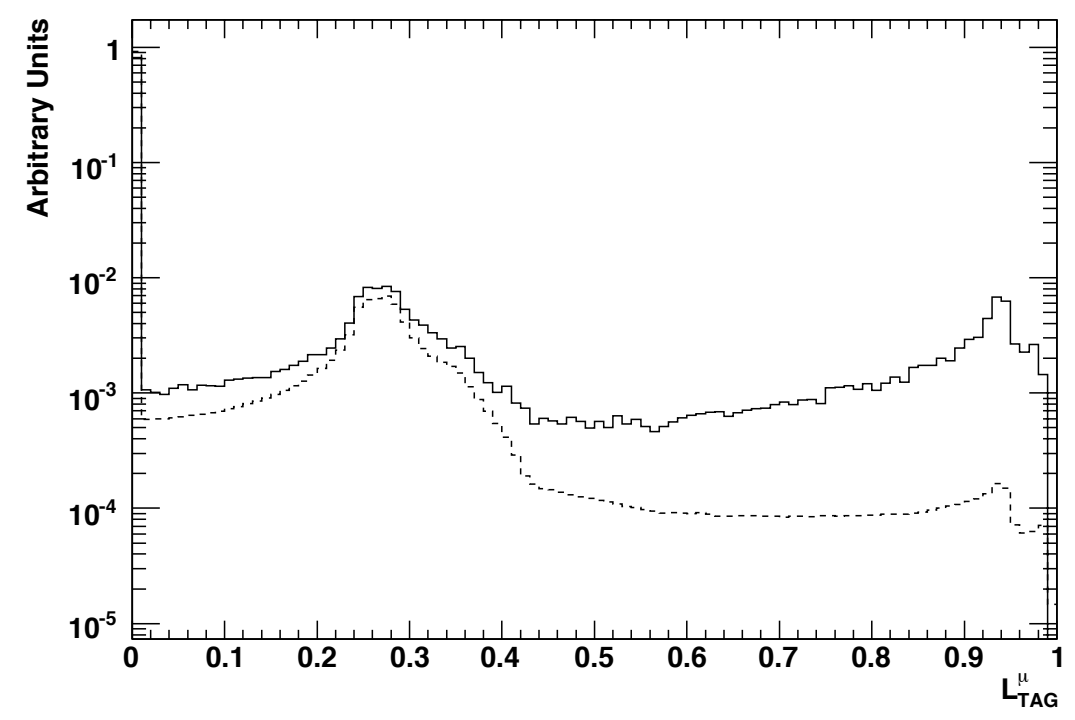

Figure 3.9: Normalised distributions of $L_{T A G}^{\mu}$ for continuum (dashed line) and correctly reconstructed candidates (solid line) before post-reconstruction cuts. Note the $\log$ scale. 


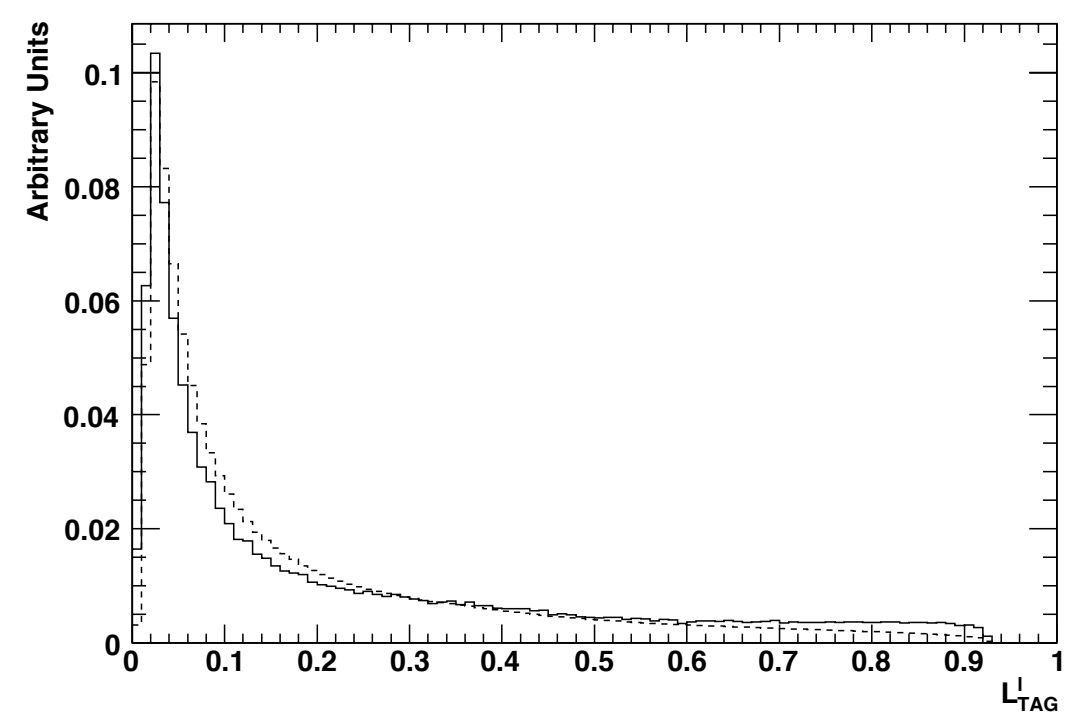

Figure 3.10: Normalised distributions of $L_{T A G}^{l}$ for continuum (dashed line) and correctly reconstructed candidates (solid line) before post-reconstruction cuts.

electron track momentum and the event missing momentum. Figure 3.8 shows the output of this classifier for simulated data and compares the distributions for correctly reconstructed candidates and continuum backgrounds. In events where the tag $B$ meson is correctly identified the output peaks at one for direct decays with a secondary peak at $\sim 0.35$ due to cascade decays. The large peak at zero represents events where no electron or positron were identified. The output is typically multiplied by the charge of the track to separate electron and positrons when identifying flavour although this is not shown here. It can be seen that there is an order of magnitude difference in the sub-tagger giving a positive identification of direct leptonic decays between these data. The presence of the secondary peak in the continuum distribution is mainly a consequence of semileptonic $D$ meson decays in these data. A similar sub-tagger, $L_{T A G}^{\mu}$, has been trained for events where any track in the ROE is identified as a muon. Figure 3.9 shows the output of the muon sub-tagger for the same data. The structure of these distributions distinguishes muons from direct and cascade decays in the same way as the electron sub-tagger. Again a significant difference in positive classifier output is observed. A final lepton sub-tagger, $L_{T A G}^{l}$, is trained using the same kinematic variables for tracks not identified as leptons in an attempt to recover any flavour information lost due to PID inefficiencies. The most lepton-like track is selected by this classifier, the output of which is shown for simulated data in figure 3.10. A value closer to one is observed for leptons which have been correctly recovered. For this sub-tagger the discrimination between signal and continuum data is not as significant; however, a residual difference at higher output values is still observed. 


\subsubsection{Hadron Tagging}

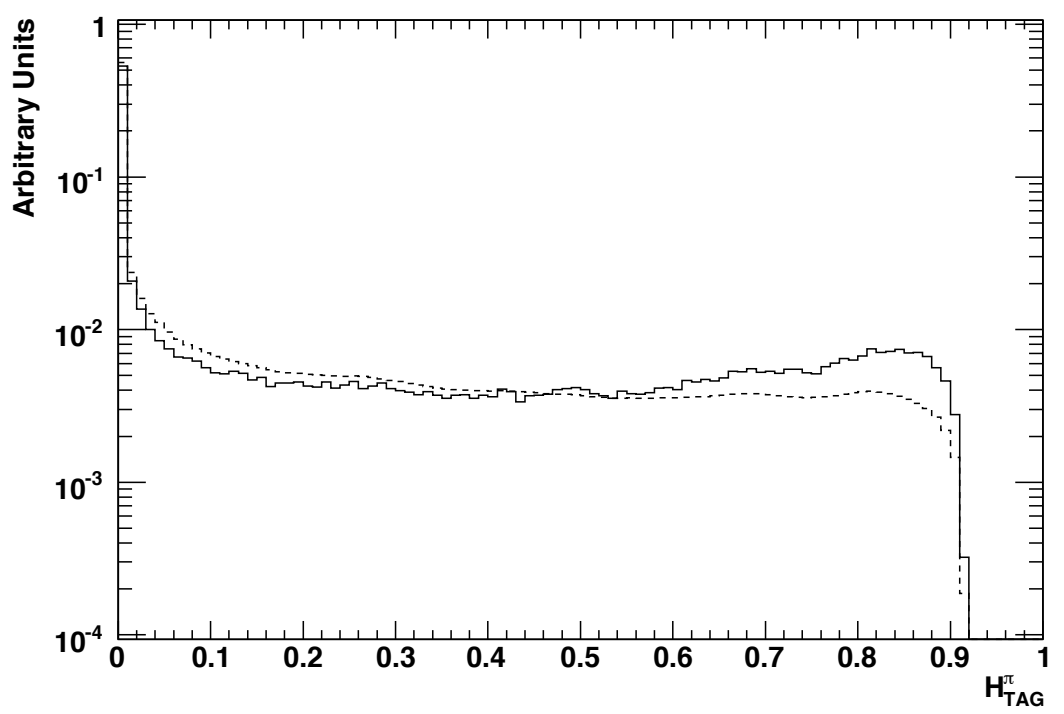

Figure 3.11: Normalised distributions of $H_{T A G}^{\pi}$ for continuum (dashed line) and correctly reconstructed candidates (solid line) before post-reconstruction cuts. Note the log scale.

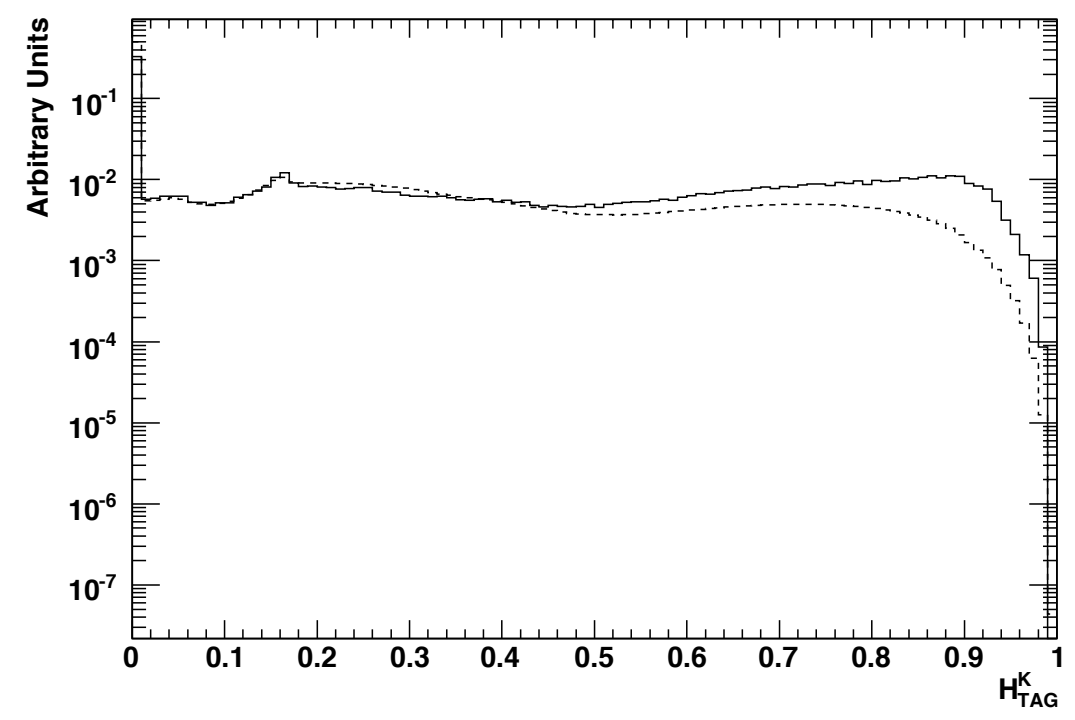

Figure 3.12: Normalised distributions of $H_{T A G}^{K}$ for continuum (dashed line) and correctly reconstructed candidates (solid line) before post-reconstruction cuts. Note the log scale.

Decays of $B$ mesons containing a $D^{* \pm}$ will often have an associated low momentum charged pion from the subsequent $D^{* \pm}$ decay. This pion will always have opposite sign charge to the parent $b$ quark. The slow pion sub-tagger, $H_{T A G}^{\pi}$, makes use of this physics 


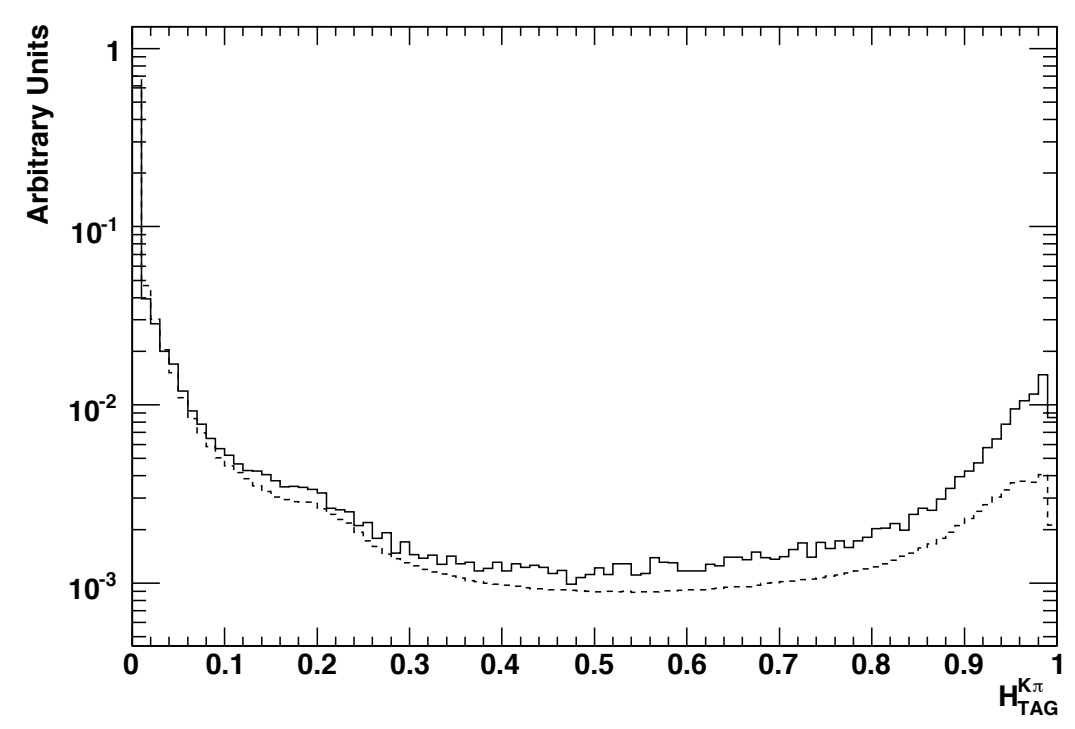

Figure 3.13: Normalised distributions of $H_{T A G}^{K \pi}$ for continuum (dashed line) and correctly reconstructed candidates (solid line) before post-reconstruction cuts. Note the $\log$ scale.

signature by identifying tracks of low CM momentum correlated with the thrust axis of all other tracks and neutrals from the ROE in that frame. Two variables describing these kinematics, along with a kaon PID classifier output to reject low momentum charged kaons, are combined in a neural net, the output of which is shown in figure 3.11 for correctly reconstructed and continuum candidates. The peak at 0.9 is observed for candidates where a low momentum track was consistent with the physics hypothesis, while the peak at zero is due to candidates with either no suitable low momentum track in the ROE or where the low momentum track identified was not consistent with a pion from a $D^{* \pm}$ decay. A similar kaon sub-tagger, $H_{T A G}^{K}$, identifies charged kaons from $b \rightarrow c \rightarrow s$ transitions which will have the same charge sign as the parent $b$ quark. This tagger combines kaon PID information and the transverse momentum properties of candidate tracks. The kaon sub-tagger distribution is shown in figure 3.12; for signal decays it peaks near one when kaons from such transitions are identified in the ROE. Outputs of the kaon and slow pion sub-taggers along with information of the angular separation of the identified pions and kaons are combined into a neural net to form a 'kaon slow pion' sub-tagger, $H_{T A G}^{K \pi}$. This sub-tagger aims to take advantage of the correlation between charge sign expected for both input sub-taggers for a true $D^{* \pm}$ decay from the $\operatorname{tag} B$ meson. The output of the kaon slow pion sub-tagger is shown in figure 3.13. The peak at one for signal indicates a correctly identified tag $B$ meson. For each hadronic sub-tagger there is on average an excess in positive output from signal decays compared to continuum events; however, this is not as significant as the difference observed in the 
electron and muon sub-tagger outputs. This is because continuum events can contain pions and kaons with similar kinematics to the $D^{* \pm}$ daughters in a $B$ meson decay chain; however, their angular distributions relative to the thrust axis are typically different due to the continuum jet-like nature resulting in the limited discrimination observed.

\subsubsection{Momentum Tagging}

The final sub-tagger, $P_{T A G}$, is used to identify high momentum particles, such as high momentum pions from $B \rightarrow D^{*} \pi$ decays. High energy tracks typically originate from the primary $B$ meson decay and tend to have the same sign charge as the parent $b$ quark. They will also have a small transverse impact parameter due to their prompt production and their direction will be correlated with that of the tag $B$ meson as they carry away a large proportion of its momentum. The high momentum sub-tagger is a neural net with three inputs reflecting these expected physical properties. The sub-tagger output is shown in figure 3.14, a higher output indicates the presence of a high momentum track in the ROE while the absence of such a particle is reflected in the peak at zero. For continuum events it can be seen that the output is typically less than for signal events and the discriminatory power between these event types is similar to that of the hadronic sub-taggers.

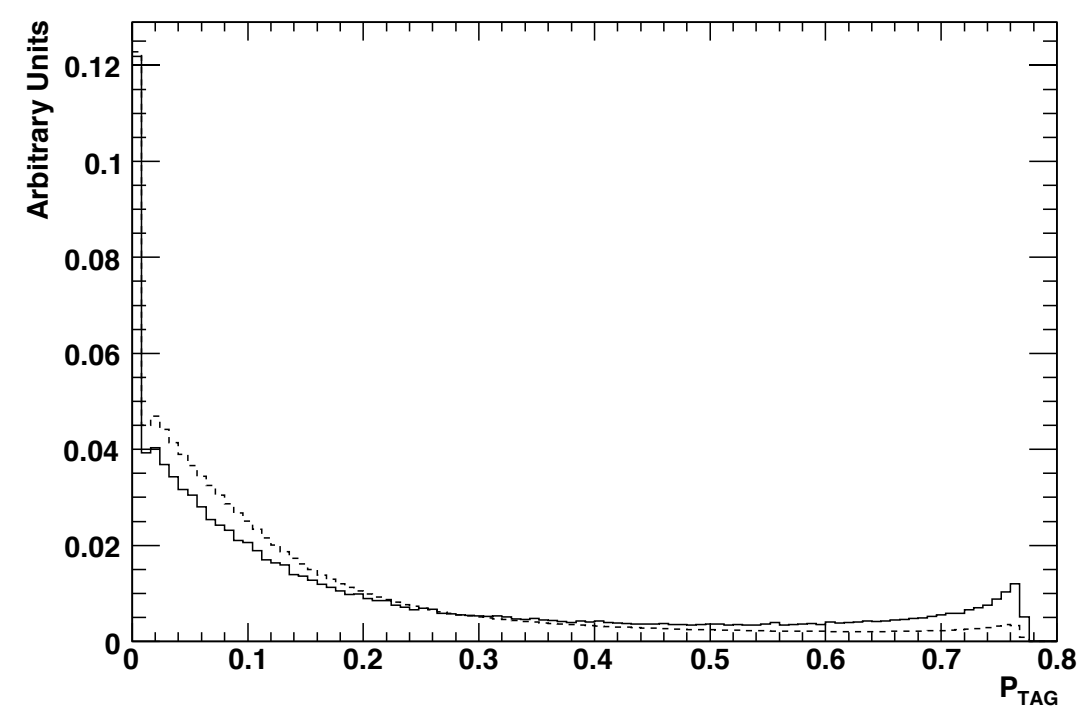

Figure 3.14: Normalised distributions of $P_{T A G}$ for continuum (dashed line) and correctly reconstructed candidates (solid line) before post-reconstruction cuts. 


\subsection{Event Reduction Filters}

As a consequence of computer processing and storage limitations, pre-reconstruction event filters ${ }^{4}$ must be applied to both experimental and MC data before full event reconstruction. General event filters are called from the BABAR framework. The BGFMultiHadron [58] passes events which have at least three charged tracks and $R_{2}<0.98$ for all charged tracks, essentially eliminating Bhabha scattering backgrounds. Events are then tested for consistency with criteria required for $B$ counting [45] through the filter BCountMHFilter [58]. A custom filter developed for previous $B A B A R$ analyses studying $B \rightarrow \rho \gamma$ transitions, BtoRhoGammaBToXGF [59], requires that for all particles in an event $R_{2}<0.9$ and that the highest energy neutral cluster in the EMC has CM energy in the range $1.15<E^{*}<3.5 \mathrm{GeV}$. All events passing these filters are then passed through a custom filter developed for this analysis, BToXdGammaFilter.

\subsubsection{BToXdGammaFilter}

The filter BToXdGammaFilter is optimised to efficiently reject backgrounds and accept simulated data events without exceeding centrally assigned processing constraints for both MC and experimental data. In each event $B$ meson candidates are reconstructed for all $B \rightarrow X_{d} \gamma$ modes listed in table 3.1. The hadronic $X_{d}$ components are reconstructed combining tracks from GoodTracksLoose, $\pi^{0}$ candidates from piODefaultMass and $\eta$ candidates from etaggDefault with all candidates from these lists required to have momentum greater than $0.3 \mathrm{GeV} / c$ in the lab frame. Where a mode has more than one charged track, $X_{d}$ candidate decay vertices are fitted with the Cascade algorithm applying the geometric constraint and requiring the $\chi^{2}$ probability of the fit to be greater than 0.001 . Where there is only one track, $X_{d}$ candidates are constructed by adding the four-momentum of the daughter particles. The composite $X_{d}$ candidates are required to have an invariant mass less that $2.2 \mathrm{GeV} / c^{2}$.

Reconstructed $X_{d}$ candidates are combined with photons to form $B$ meson candidates. Photons, from GoodPhotonLoose, are required to have CM energy in the range $1.15<$ $E^{*}<3.5 \mathrm{GeV}$ and clusters with at least four EMC crystals over energy readout threshold to eliminate fake neutral clusters which can potentially arise due to a single noisy crystal. $B$ meson candidates are required to have $|\Delta E|<0.3 \mathrm{GeV}$ and $5.0<m_{E S}<5.3 \mathrm{GeV} / c^{2}$. The angle between the $B$ meson candidate momentum and thrust axis of the ROE is required to have an absolute cosine less than 0.8. Any event which has at least one

\footnotetext{
${ }^{4}$ Pre-reconstruction (post-reconstruction) refers to event processing carried out before (after) the custom event reconstruction described in section 3.5. All events discussed in this chapter have been reconstructed within the central BABAR framework.
} 


\begin{tabular}{|l|l|l|l|l|}
\hline MC data & Filter & Events & Relative Eff (\%) & Total Eff (\%) \\
\hline$B^{+} \rightarrow X_{d u} \gamma$ & None & $1,288,000$ & 100.0 & 100.0 \\
& BGFMultiHadron & $1,244,491$ & 96.6 & 96.6 \\
& BCountMHFilter & $1,149,425$ & 92.4 & 89.2 \\
& BtoRhoGammaBToXGF & 959,714 & 83.5 & 74.5 \\
& BToXdGammaFilter & 453,992 & 47.3 & 35.2 \\
\hline$B^{0} \rightarrow X_{d d} \gamma$ & None & $1,288,000$ & 100.0 & 100.0 \\
& BGFMultiHadron & $1,241,756$ & 96.4 & 96.4 \\
& BCountMHFilter & $1,143,968$ & 92.2 & 88.9 \\
& BtoRhoGammaBToXGF & 951,564 & 83.2 & 73.9 \\
& BToXdGammaFilter & 441,343 & 46.4 & 34.3 \\
\hline$B^{+} \rightarrow X_{s u} \gamma$ & None & $11,178,000$ & 100.0 & 100.0 \\
& BGFMultiHadron & $10,762,617$ & 96.3 & 96.3 \\
& BCountMHFilter & $9,804,116$ & 91.1 & 87.7 \\
& BtoRhoGammaBToXGF & $8,136,455$ & 83.0 & 72.8 \\
& BToXdGammaFilter & $3,293,162$ & 40.5 & 29.5 \\
\hline$B^{0} \rightarrow X_{s d} \gamma$ & None & $11,178,000$ & 100.0 & 100.0 \\
& BGFMultiHadron & $10,724,379$ & 95.9 & 95.9 \\
& BCountMHFilter & $9,726,955$ & 90.7 & 87.0 \\
& BtoRhoGammaBToXGF & $8,060,449$ & 82.9 & 72.1 \\
& BToXdGammaFilter & $3,279,972$ & 40.7 & 29.3 \\
\hline
\end{tabular}

TABLE 3.5: Efficiencies of pre-reconstruction event filters when applied to the high mass bin signal MC data.

$B$ meson candidate passing all of the above filter requirements is kept for full event reconstruction.

The choice of variables used in BToXdGammaFilter and the value of cuts on those variables are based primarily on the equivalent filter used in the previous version of this analysis [1]. However, the filter described here improves on the previous analysis through a revision of the choice of $B A B A R$ event framework track and neutral candidate lists used for hadronic candidate reconstruction. The lists described above perform tighter selections on their constituent candidates and are therefore smaller in size. This leads to a significant reduction in the potential number of combinations allowed to form hadronic candidates in a given event with an almost negligible cost in signal efficiency. The processing overhead saved allows the invariant mass cut to be relaxed from $2.0 \mathrm{GeV} / c^{2}$ to $2.2 \mathrm{GeV} / c^{2}$ therefore allowing for a potentially more inclusive measurement. Due to the required processing constraints this cut cannot be relaxed any further. 


\begin{tabular}{|l|l|l|l|l|}
\hline MC data & Filter & Events & Relative Eff $(\%)$ & Total Eff $(\%)$ \\
\hline$B^{0} \rightarrow \rho^{+} \gamma$ & None & 650,000 & 100.0 & 100.0 \\
& BGFMultiHadron & 609,153 & 93.7 & 93.7 \\
& BCountMHFilter & 508,536 & 83.5 & 78.2 \\
& BtoRhoGammaBToXGF & 443,283 & 87.2 & 68.2 \\
& BToXdGammaFilter & 323,325 & 72.9 & 49.7 \\
\hline$B^{0} \rightarrow \rho^{0} \gamma$ & None & 650,000 & 100.0 & 100.0 \\
& BGFMultiHadron & 636,882 & 98.0 & 98.0 \\
& BCountMHFilter & 539,034 & 84.6 & 82.9 \\
& BtoRhoGammaBToXGF & 450,721 & 83.6 & 69.3 \\
& BToXdGammaFilter & 364,193 & 80.8 & 56.0 \\
\hline$B^{0} \rightarrow \omega \gamma$ & None & 650,000 & 100.0 & 100.0 \\
& BGFMultiHadron & 622,903 & 95.8 & 95.8 \\
& BCountMHFilter & 513,547 & 82.4 & 79.0 \\
& BtoRhoGammaBToXGF & 439,831 & 85.6 & 67.7 \\
& BToXdGammaFilter & 318,105 & 72.3 & 48.9 \\
\hline$B^{0} \rightarrow K^{*+} \gamma$ & None & $6,449,000$ & 100.0 & 100.0 \\
& BGFMultiHadron & $6,082,909$ & 94.3 & 94.3 \\
& BCountMHFilter & $5,059,206$ & 83.2 & 78.4 \\
& BtoRhoGammaBToXGF & $4,277,607$ & 84.6 & 66.3 \\
& BToXdGammaFilter & $2,136,768$ & 50.0 & 33.1 \\
& None & $6,449,000$ & 100.0 & 100.0 \\
& BGFMultiHadron & $6,047,163$ & 93.8 & 93.8 \\
& BCountMHFilter & $4,938,077$ & 81.7 & 76.6 \\
\hline$B^{0} \rightarrow K^{* 0} \gamma$ & BToXhoGammaBToXGF & $4,149,053$ & 84.0 & 42.9 \\
& & $2,764,181$ & 66.6 & \\
& & & \\
& & & \\
& & & \\
& &
\end{tabular}

TABLE 3.6: Efficiencies of pre-reconstruction event filters when applied to the low mass bin signal MC data.

\begin{tabular}{|l|l|l|}
\hline MC data & Events & Eff $(\%)$ \\
\hline$B^{+} \rightarrow X_{d u} \gamma$ & 412,189 & 34.3 \\
$B^{0} \rightarrow X_{d d} \gamma$ & 398,935 & 33.2 \\
$B^{+} \rightarrow X_{s u} \gamma$ & $3,042,628$ & 29.2 \\
$B^{0} \rightarrow X_{s d} \gamma$ & $2,983,045$ & 28.6 \\
\hline
\end{tabular}

TABLE 3.7: High mass bin signal MC samples after filter and hadronic mass cuts. The efficiency is relative to the pre-filter samples given in table 3.3. 


\begin{tabular}{|l|l|l|}
\hline MC data & Events & Eff $(\%)$ \\
\hline$B^{0} \rightarrow \rho^{+} \gamma$ & 282,157 & 49.6 \\
$B^{0} \rightarrow \rho^{0} \gamma$ & 317,817 & 55.8 \\
$B^{0} \rightarrow \omega \gamma$ & 318,105 & 48.9 \\
$B^{0} \rightarrow K^{*+} \gamma$ & $1,889,573$ & 32.8 \\
$B^{0} \rightarrow K^{* 0} \gamma$ & $2,441,107$ & 42.6 \\
\hline
\end{tabular}

TABLE 3.8: Low mass bin signal MC samples after filter and hadronic mass cuts. The efficiency is relative to the pre-filter samples given in table 3.4.

\begin{tabular}{|l|l|l|l|}
\hline Type & Pre Filter Events & Post Filter Events & Efficiency (\%) \\
\hline Generic $B^{+} B^{-} \mathrm{MC}$ & $708,762,000$ & $1,623,556$ & 0.23 \\
Generic $B^{0} \bar{B}^{0} \mathrm{MC}$ & $717,995,000$ & $1,081,134$ & 0.15 \\
Generic $c \bar{c} \mathrm{MC}$ & $1,128,544,000$ & $9,642,971$ & 0.85 \\
Generic $q \bar{q} \mathrm{MC}(q=u, d, s)$ & $1,670,948,000$ & $27,897,989$ & 1.68 \\
On Peak Data & $6,714,057,036$ & $20,033,960$ & 0.30 \\
Off Peak Data & $642,952,515$ & $1,932,935$ & 0.30 \\
\hline
\end{tabular}

TABLE 3.9: Pre-reconstruction filter efficiencies for generic MC backgrounds and experimental data.

\subsubsection{Filter Efficiencies}

The relative and cumulative efficiencies of the above filters when applied to the signal MC data listed in table 3.2 are shown in tables 3.5 and 3.6 for high mass bin and low mass bin samples respectively. After the filters are applied the model cut on hadronic mass due to $m_{X}^{T}=1.0 \mathrm{GeV} / c^{2}$ must be made to the filtered samples. Tables 3.7 and 3.8 show the effect of this cut for the high and low mass bin samples respectively; the efficiencies quoted in these tables are the effective combined filter efficiency of each sample for events which satisfy the mass threshold requirement. Table 3.9 shows the total combined efficiency of all filters when applied to the generic MC data samples listed in table 3.2 and to the experimental data.

The combined signal efficiencies appear low due to low efficiency for events where the signal decay does not directly correspond to one of the reconstructed modes listed in table 3.1 .

\subsection{Full Event Reconstruction}

Full event reconstruction is performed for all events passing the pre-reconstruction filters. Hadronic candidates are created with identical lists and using the same vertex fitting 
strategy as the BToXdGammaFilter event filter. However, the laboratory momentum cut is relaxed to $0.1 \mathrm{GeV} / c$, the vertex $\chi^{2}$ probability cut is not applied and candidates can have a hadronic mass up to $2.4 \mathrm{GeV} / c^{2}$. The relaxation of these cuts is temporary and a historical artifact of the code inherited from the previous version of this analysis [1]; they are tightened again to reflect the filter cuts in the subsequent post-reconstruction event selection. Additionally where there was only one charged final state particle the candidates are formed by adding the daughter four-momenta constraining all daughters to originate from the event primary vertex.

Before hadronic candidates are fitted a PID selection is applied to identify them as either $X_{d}$ or $X_{s}$ candidates. For each track in GoodTracksLoose considered for hadronic candidate reconstruction the PID selectors pionKMLoose and kaonBDTNotAPion are called. If a track passes both or neither selectors it is not considered for reconstruction. Tracks passing only the pion selector are assumed to be pions. Tracks passing only the kaon selector are assumed to be kaons and their four-momentum is redefined by assigning them the world average kaon mass [14]. During hadronic candidate reconstruction if all tracks are identified as pions the candidate is classified as a $X_{d}$ and fitted. If all but one track is a pion and the remaining track a kaon with correct sign charge where relevant, the candidate is classified as a $X_{s}$ and fitted. Any other combination of tracks results in the candidate being vetoed from the reconstruction. Fitted hadronic candidates satisfying the reconstruction criteria are combined with photons from GoodPhotonLoose applying the same CM frame energy cut on the photon as in BToXdGammaFilter to form $B$ meson candidates. Again the addition of these four momenta are constrained such that the $X_{d}$ candidate and photon candidate originate from the event primary vertex. $B$ meson candidates are required to have $m_{E S}^{\prime}$ greater than $5.0 \mathrm{GeV} / c^{2}$ and $|\Delta E| \leq 0.3 \mathrm{GeV}$. A further cut of $\left|\cos \theta_{T}\right| \leq 0.8$ is then applied to reduce continuum backgrounds in order to save disk space and reduce processing time in subsequent event selection. This cut does not exactly mirror the similar cut made in BToXdGammaFilter as here the angle is defined as the thrust axis relative to the photon direction as opposed to the reconstructed $B$ meson direction; however, these distributions are similar as the background photon direction is generally correlated with jet momentum in continuum events whereas signal photon momentum direction will on average reflect the isotropic nature of signal events. For each reconstructed $B$ meson candidate satisfying the above requirements kinematic, topological and tagging variable information is saved for subsequent analysis. Table 3.10 shows the total number of events containing at least one $B$ meson candidate after reconstruction for both $\mathrm{MC}$ and experimental data. 


\begin{tabular}{|l|l|l|l|l|}
\hline Data Type & $\geq 1$ Cand & Eff $(\%)$ & $\geq 1 X_{d}$ Cand & $\geq 1 X_{s}$ Cand \\
\hline$X_{d u} \gamma$ signal MC & 334,022 & 81.0 & $287,813(90,200)$ & $152,148(2,476)$ \\
$X_{d d} \gamma$ signal MC & 325,232 & 81.5 & $287,625(60,822)$ & $134,698(3,016)$ \\
$X_{s u} \gamma$ signal MC & $2,374,495$ & 78.0 & $1,275,415(47,203)$ & $1,808,478(584,981)$ \\
$X_{s d} \gamma$ signal MC & $2,341,138$ & 78.5 & $1,256,499(29,861)$ & $1,772,401(486,733)$ \\
$\rho^{+} \gamma$ signal MC & 249,863 & 88.6 & $237,867(147,054)$ & $90,279(3,957)$ \\
$\rho^{0} \gamma$ signal MC & 272,947 & 85.9 & $258,797(197,827)$ & $80,571(10,809)$ \\
$\omega \gamma$ signal MC & 273,002 & 85.8 & $258,090(46,600)$ & $101,574(1,552)$ \\
$K^{*+} \gamma$ signal MC & $1,574,018$ & 83.3 & $798,196(109,072)$ & $1,135,465(466,752)$ \\
$K^{* 0} \gamma$ signal MC & $2,044,754$ & 83.8 & $644,443(30,181)$ & $1,806,368(1,323,321)$ \\
Generic $B^{+} B^{-} \mathrm{MC}$ & $1,044,389$ & 64.3 & 553,997 & 697,965 \\
Generic $B^{0} \bar{B}^{0} \mathrm{MC}$ & 709,728 & 65.6 & 390,398 & 463,011 \\
Generic $c \bar{c} \mathrm{MC}$ & $6,490,155$ & 67.3 & $4,085,150$ & $3,799,608$ \\
Generic $q \bar{q} \mathrm{MC}$ & $19,001,795$ & 68.1 & $15,163,515$ & $6,284,873$ \\
On Peak Data & $13,702,098$ & 68.4 & $9,976,160$ & $5,790,300$ \\
Off Peak Data & $1,323,267$ & 68.5 & 974,466 & 541,629 \\
\hline
\end{tabular}

TABLE 3.10: Events with at least one reconstructed candidate after full event reconstruction for both MC and experimental data and the reconstruction efficiency relative to the number of events passing pre-reconstruction event filters given in tables 3.7, 3.8 and 3.9. Also shown are the number of events with at least one fitted $X_{d}$ candidate and the number of events with at least one fitted $X_{s}$ candidate. Shown in parentheses are the number of events where a correctly reconstructed candidate is identified. Note that the PID is not required to be correct for a candidate to be considered correctly reconstructed.

\subsubsection{Signal Candidate Identification}

When considering signal MC data the identification of correctly reconstructed events is vital to estimate the combinatoric background contributions from reconstructing and selecting candidates within these data. Due to contamination from beam backgrounds and detector noise there is no one-to-one correspondence between particles generated in underlying physics processes and those in the reconstructed lists available after the detector simulation has run. For this reason the BABAR simulation framework uses objects known as 'gHits' for each simulated particle to model its interaction with active detector elements. Detector responses such as DCH hits and EMC crystal readouts can have one, many or no gHits associated with them, depending on the origin of the interaction and the simulated efficiencies of that particular subsystem. The number of gHits present in reconstructed tracks and clusters allow the user to find consistency between that reconstructed candidate and any generated particle.

This analysis has two dominant combinatoric backgrounds in signal MC data. Firstly a candidate whose reconstructed mode matches that of the generated event but which 
uses one or more tracks and clusters from the ROE in the fit. Secondly a candidate whose reconstructed mode does not match the generated mode. Of the latter there are those candidates where the generated mode was one of the other signal modes from table 3.1 and those where the generated mode was some other allowed $B \rightarrow X_{s / d} \gamma$ transition. No significant contribution is observed from candidates where the hadronic component is correctly reconstructed but an incorrect photon is used to form the $B$ meson. Combinatoric backgrounds are therefore dominated by the reconstruction of the hadronic candidate.

During the reconstruction of each signal event a list of the generated particles is used to identify the true decay mode. If the generated mode is a signal mode and there is a reconstructed candidate of the same mode then the gHit consistency associator is used to identify if the hadronic component was correctly reconstructed. The associator is called for each reconstructed track and for each cluster used to create a $\pi^{0}$ or $\eta$. If all reconstructed candidates are associated with a unique generated charged pion, charged kaon or photon from the true signal $B$ meson then the hadronic component and its corresponding $B$ meson are considered to be correctly reconstructed.

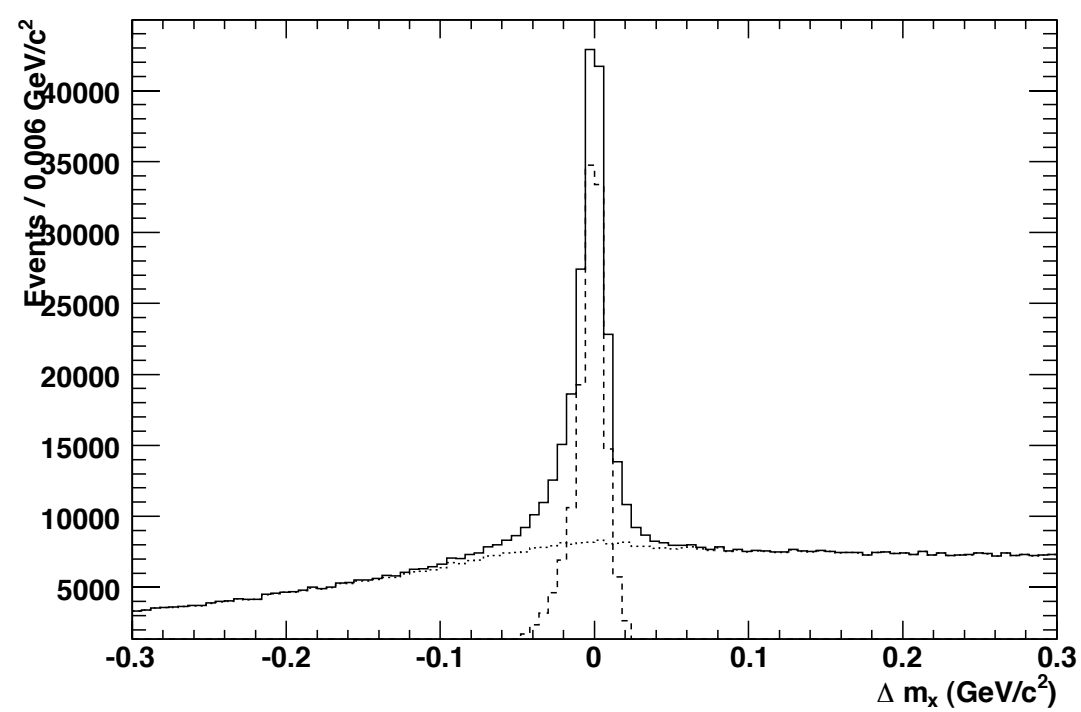

Figure 3.15: Distribution of $\Delta m_{X}$ for $X_{d}$ candidates whose reconstructed mode matches the generated mode in high mass $B \rightarrow X_{d} \gamma$ simulated events. The dashed line shows candidates identified as correctly reconstructed by gHit association and the dotted line shows the contribution from all other candidates where the reconstructed decay mode matches the generated decay mode.

To investigate the gHit associator method for inefficiencies an independent cross check is carried out. The difference between the reconstructed and generated hadronic mass, $\Delta m_{X}$, for candidates whose reconstructed mode matches the generated mode of an 
event will peak at zero with a narrow spread due to detector resolution for correctly reconstructed candidates, while no significant narrow peak will exist for background candidates of that mode. Figure 3.15 shows the distribution of $\Delta m_{X}$ for $B \rightarrow X_{d} \gamma$ signal MC candidates where the reconstructed mode matches the generated mode. It can be seen that candidates that fail the gHit associator do not show a significant peak at zero. It is therefore assumed that for the purposes of this analysis the associator efficiently identifies correctly reconstructed candidates.

\subsection{Post Reconstruction Event Reduction}

Combinatoric backgrounds dominate over events with correctly reconstructed candidates after full event reconstruction. In order to maximise the statistical significance of correctly reconstructed events a number of variables are identified to which cuts can be applied. The following section describes the variables used and details how the optimal values for each cut were determined.

\subsection{1 $\quad B \rightarrow X_{s} \gamma$ Background from $K_{S}^{0}$ Decays}

In both the high and low mass region, $B \rightarrow X_{d} \gamma$ candidate decays for certain modes will have hadronic final states identical to $B \rightarrow X_{s} \gamma$ decays where there was an intermediate $K_{S}^{0} \rightarrow \pi^{+} \pi^{-}$or $K_{S}^{0} \rightarrow \pi^{0} \pi^{0}$ decay. For example the decay $B^{0} \rightarrow X_{d} \gamma \rightarrow \pi^{+} \pi^{-} \pi^{0} \gamma$ is identical to the decay $B^{0} \rightarrow X_{s} \gamma \rightarrow K_{S}^{0}\left(\pi^{+} \pi^{-}\right) \pi^{0} \gamma$. Such backgrounds can contribute in all reconstructed $B \rightarrow X_{d} \gamma$ modes with three or more final state particles from the hadronic decay.

The background from $K_{S}^{0} \rightarrow \pi^{+} \pi^{-}$decays can be reduced by considering the decay length and invariant mass of potential $K_{S}^{0}$ candidates. During event reconstruction all $X_{d}$ candidates with two or more charged pions have their vertex reconstructed with the Cascade algorithm which determines the most probable position for the decay vertex of that candidate. Figure 3.16 shows the distance between the decay vertex and event primary vertex in the laboratory frame, $x_{D E C}$, for both correctly reconstructed $B \rightarrow$ $X_{d} \gamma$ candidates and correctly reconstructed $B \rightarrow X_{s} \gamma$ decays whose final states are indistinguishable due an intermediate $K_{S}^{0} \rightarrow \pi^{+} \pi^{-}$decay. It can be seen that the $K_{S}^{0}$ backgrounds have a significant tail at high values of $x_{D E C}$, not seen in signal events. This can be attributed to the relatively large decay length of the $K_{S}^{0}$ meson [14]. During event reconstruction pairs of oppositely charged tracks from GoodTracksLoose which pass the PID selector PionKMLoose (and therefore which can potentially be used in $X_{d}$ candidate reconstruction) are also separately vertexed with the Cascade algorithm and 


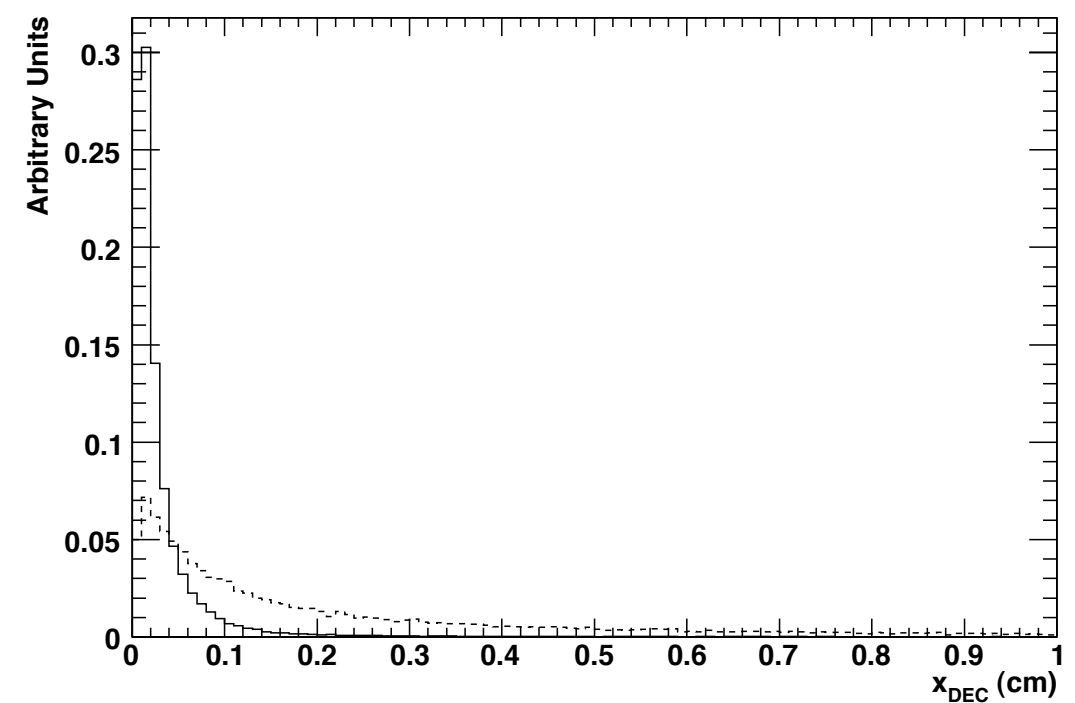

FIGURE 3.16: Distance between the decay vertex and event primary vertex for reconstructed modes with at least two charged tracks and at least three hadronic final state particles showing correctly reconstructed $B \rightarrow X_{d} \gamma$ candidates (solid line) and correctly reconstructed $B \rightarrow X_{s} \gamma$ background candidates which have indistinguishable final states due to an intermediate $K_{S}^{0} \rightarrow \pi^{+} \pi^{-}$decay (dashed line).

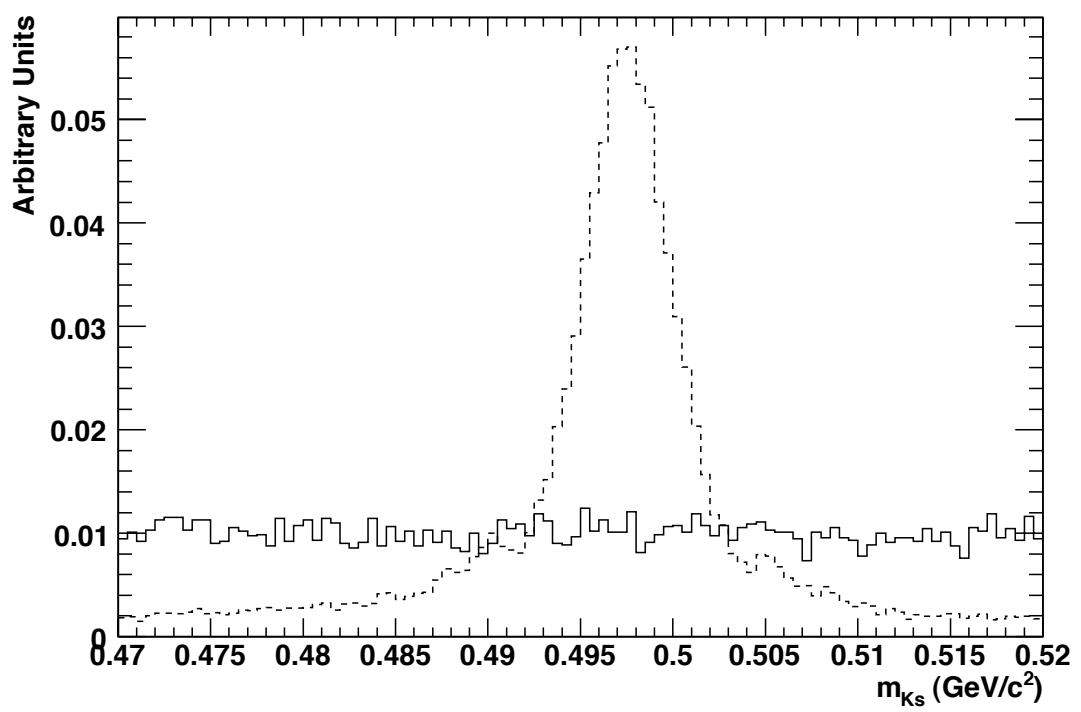

FiguRE 3.17: Invariant mass of reconstructed $K_{S}^{0} \rightarrow \pi^{+} \pi^{-}$candidates where both charged tracks are also used to reconstruct a correctly identified $B \rightarrow X_{d} \gamma$ candidate (solid line) or a correctly identified $B \rightarrow X_{s} \gamma$ background candidate with an intermediate $K_{S}^{0} \rightarrow \pi^{+} \pi^{-}$decay (dashed line) 
saved. Figure 3.17 shows the the invariant mass of such track pairs, $m_{K_{s}}$, when both tracks are also used in the reconstruction of a signal $B \rightarrow X_{d} \gamma$ or the reconstruction of a correctly identified $B \rightarrow X_{s} \gamma$ background with intermediate $K_{S}^{0} \rightarrow \pi^{+} \pi^{-}$decay. The background shows a clear peak about the $K_{S}^{0}$ mass whereas those candidates where the pion pair came from a signal decay have a uniform distribution. Requiring that no $X_{d}$ candidate with greater than three hadronic final state particles (of which at least two were charged pion candidates) is reconstructed from any pair of charged tracks which were also used to reconstruct a $K_{S}^{0}$ candidate with $0.485 \leq m_{K_{s}} \leq 0.51 \mathrm{GeV} / c^{2}$ and that the $X_{d}$ candidate has $x_{D E C} \leq 0.2 \mathrm{~cm}$ is found to reject $>95 \%$ of $K_{S}^{0} \rightarrow \pi^{+} \pi^{-}$ backgrounds while rejecting $<5 \%$ of signal candidates whose modes are subject to this background. For ease of technical implementation the cut on $x_{D E C}$ is also applied to mode 1 of table 3.1 at the cost of $<5 \%$ of signal candidates from that mode before further post reconstruction cuts are applied.

Backgrounds from $K_{S}^{0} \rightarrow \pi^{0} \pi^{0}$ are less straight forward to reduce. This background only contains neutral particles so it is not possible to obtain reliable vertex information for the $K_{S}^{0}$ decay. Consequently, as $\pi^{0}$ candidates in PiODefaultmass are defined to originate from the primary vertex, the mass resolution of any reconstructed $K_{S}^{0}$ candidate will be much worse than those reconstructed from charged track pairs. Time constraints on the analysis meant no study was carried out to reduce this background, the consequences of which are discussed in section 3.6.7.1.

\subsubsection{High Energy Photon Candidate}

The dominant background for high energy photon candidates are asymmetric $\pi^{0} \rightarrow \gamma \gamma$ and $\eta \rightarrow \gamma \gamma$ processes where one photon carries away most of the energy from the decay. To reduce these backgrounds every high energy photon candidate used to reconstruct a $B$ meson has its four-momentum added to all other photons in the event with lab energy greater than $30 \mathrm{MeV}(250 \mathrm{MeV})$ and the invariant mass closest to the nominal $\pi^{0}$ $(\eta)$ mass [14] is saved. Figures 3.18 and 3.19 show the $\pi^{0}$ and $\eta$ distributions respectively for combinatoric and correctly reconstructed MC events before post-reconstruction cuts are applied. During cut optimisation an invariant mass veto window is considered with each veto consisting of an upper and lower cut.

The lateral moment cut required for a cluster to be included in the GoodPhotonLoose (see section 3.2.2) list is tightened so that only photons with lateral moment less than 0.6 are considered. Other radiative penguin analyses using BABAR data have shown that this cut value has almost negligible signal rejection [59] and so it is implemented here as well. 


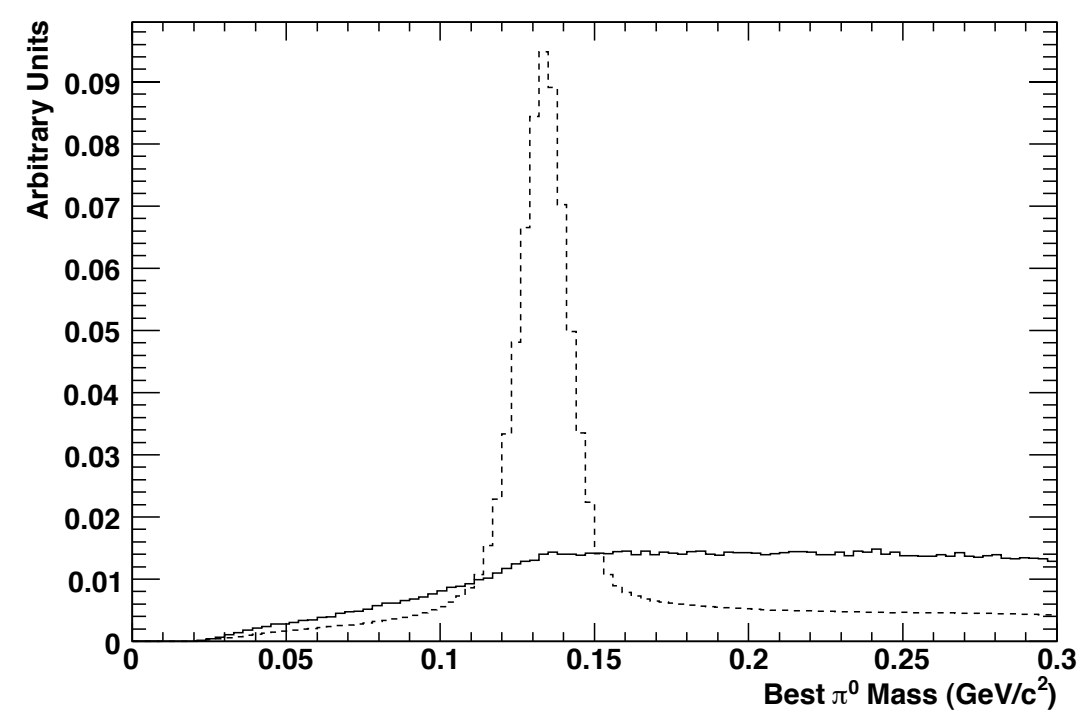

Figure 3.18: Normalised distributions of the invariant mass closest to the $\pi^{0}$ mass from combining the high energy photon with other photons of energy $>30 \mathrm{MeV}$ for continuum (dashed line) and correctly reconstructed candidates (solid line) before postreconstruction cuts.

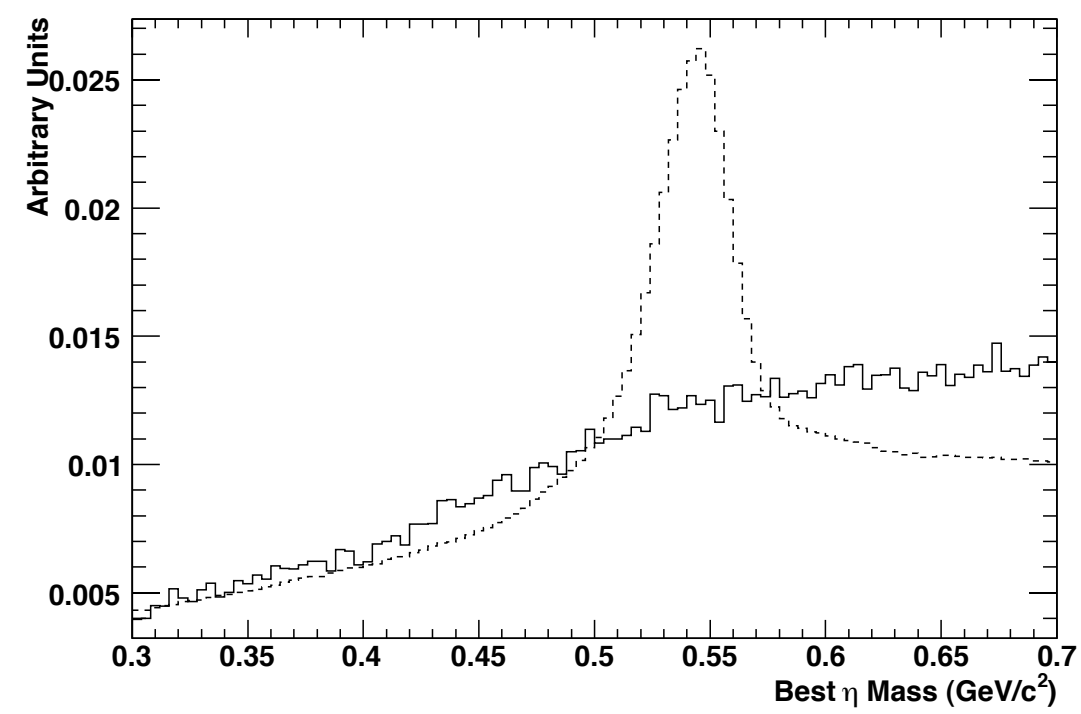

Figure 3.19: Normalised distributions of the invariant mass closest to the $\eta$ mass from combining the high energy photon with other photons of energy $>250 \mathrm{MeV}$ for continuum (dashed line) and correctly reconstructed candidates (solid line) before postreconstruction cuts. 


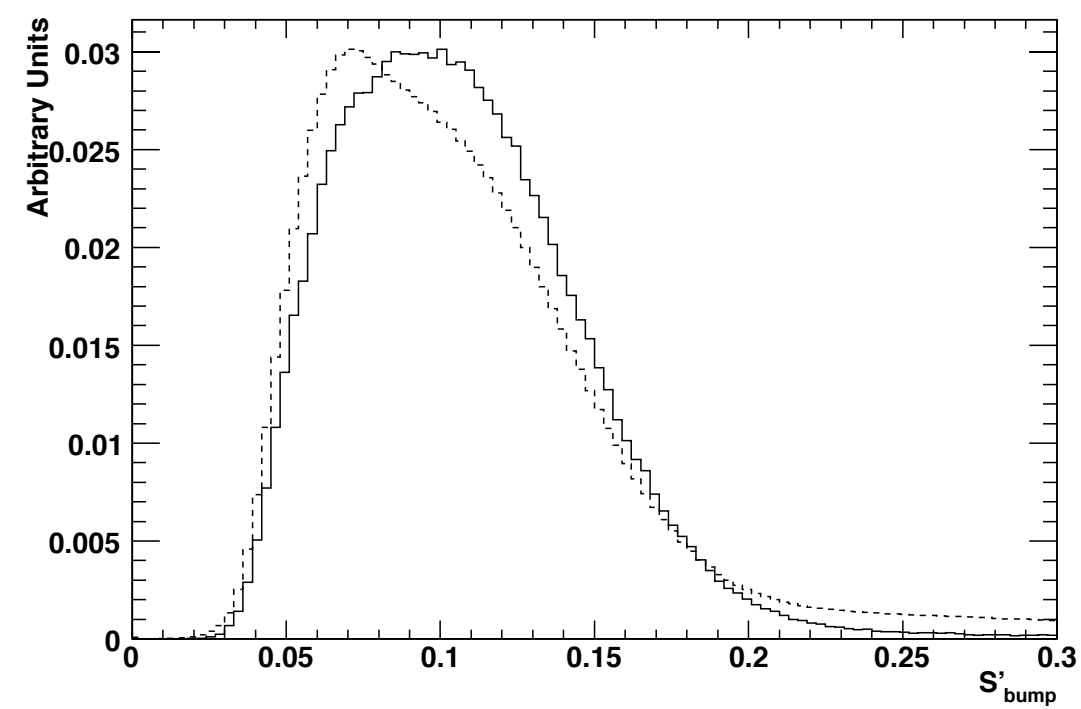

Figure 3.20: Normalised distributions of $S_{\text {bump }}^{\prime}$ for continuum (dashed line) and correctly reconstructed candidates (solid line) before post reconstruction cuts.

Backgrounds from merged $\pi^{0} \rightarrow \gamma \gamma$ decays where both photons form the same cluster in the EMC can be rejected by considering the second moment of the cluster, $S_{\text {bump }}$. From equation (2.5) it can be seen that $S_{\text {bump }}$ is geometrically dependent on the position of a cluster in the EMC. It is therefore more useful to multiply $S_{\text {bump }}$ by the distance from the IR to the cluster centroid, giving a corrected second moment, $S_{b u m p}^{\prime}$, independent of the geometry. Figure 3.20 shows the distributions of $S_{b u m p}^{\prime}$ for combinatoric and correctly reconstructed candidates before cuts are applied and it is this value for which a cut is considered. The enhanced high end tail in the combinatoric distribution can be mainly attributed to merged $\pi^{0}$ decays.

A further background which can contribute fake high energy photons arises from interactions between high energy charged particles and detector material whereby scattered neutrons can deposit energy in the EMC. If that cluster is then mistakenly matched with the charged track, the actual energy deposit from the charged particle will be assigned as a neutral particle. To reduce these backgrounds the distance from the candidate photon cluster to the nearest cluster associated with a charged track, $x_{t r k}$, is a useful discriminant. An isolation cut requiring $x_{t r k} \geq 25 \mathrm{~cm}$ is applied based on the equivalent cut used in previous radiative penguin analyses using $B A B A R$ data $[1,59]$.

To remove possible backgrounds from single noisy crystals in data the EMC cluster is required to have greater than four constituent crystals. Additionally there must be no dead or damaged crystals within the cluster to ensure the energy measurement is as accurate as possible. 
Finally ISR background photons typically have low transverse momentum so are largely found in the endcap. Therefore requiring that reconstructed photons only have associated clusters whose centroid is in the barrel of the EMC can reduce these backgrounds. Rejection of endcap photons is thus a binary variable and is considered in the cut optimisation discussed below.

\subsection{3 $\quad X_{s / d}$ Candidate Cuts}

To further reduce combinatoric backgrounds cuts can be placed on reconstructed hadronic $X_{s / d}$ candidates. For those modes with more than one charged track a cut is placed on the $\chi^{2}$ probability of the vertex fit. The cut value is based on the experience of previous radiative penguin analyses where multiple track final states are reconstructed $[1,30]$ and is required to be greater than $1 \%$ for all such candidates.

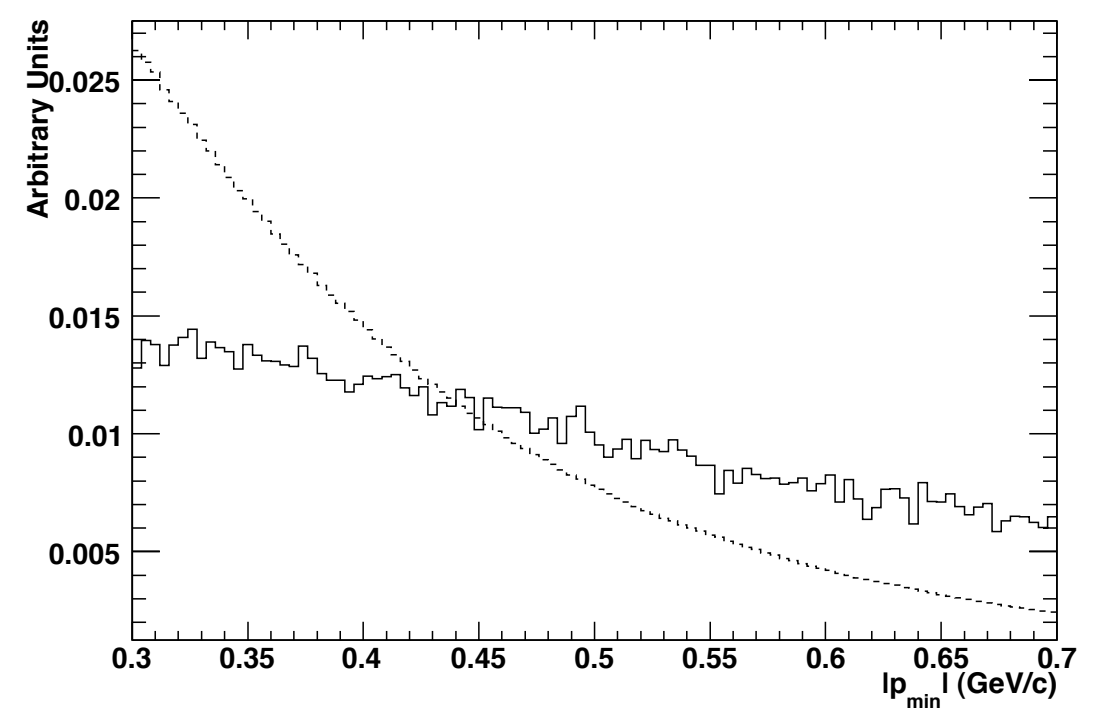

FIGURE 3.21: Normalised distributions of the momentum magnitude of the lowest momentum hadronic daughter for continuum (dashed line) and correctly reconstructed candidates (solid line) before post reconstruction cuts.

The cut on the laboratory momentum of daughters of the $X_{s / d}$ candidate, $\left|p_{\min }\right|$, is tightened during cut optimisation with the requirement that it must be at least as tight as the value of $0.3 \mathrm{GeV} / c$ implemented in the filter BToXdGammaFilter. Figure 3.21 shows distributions of this variable comparing correctly reconstructed candidates and combinatoric background for the lowest momentum daughter used to fit that candidate. Combinatoric backgrounds tend to favour lower momenta relative to correctly reconstructed candidates. 
Finally for charged pions the tightness of the PID selector imposed on tracks used to reconstruct $X_{d}$ candidates is varied to investigate which gives the best performance accepting signal and rejecting backgrounds.

\subsection{4 $B$ Meson Candidate Cuts}

It is possible to tighten cuts applied to $m_{E S}^{\prime}$ and $\Delta E$ during event selection to reduce combinatoric backgrounds. This is providing that there is no significant signal loss which might bias the subsequent fit to these variables. Therefore the analysis requires $-0.3 \leq \Delta E \leq 0.2 \mathrm{GeV}$ and $m_{E S} \geq 5.22 \mathrm{GeV} / c^{2}$ the choice of which is based on the experience of previous $B A B A R$ radiative penguin analyses $[1,30,59]$.

\subsubsection{Hadronic Mass Bin Variations}

As described above the choice of upper limit in the non-resonant hadronic mass bin is constrained by computational limitations in processing the pre-reconstruction event filters. The filter BToXdGammaFilter only reconstructs $X_{d}$ candidates. Due to the difference in pion and kaon mass it follows that $X_{s}$ candidates close to the upper limit can have significantly different efficiencies when passed through this filter. This is because the filter assumes pion mass for all charged tracks, whereas fully reconstructed $X_{s}$ candidates redefine the mass of the track identified as a kaon and therefore have an increased reconstructed hadronic mass. In post-reconstruction event reduction the upper limit is therefore not allowed to exceed $2.0 \mathrm{GeV} / c^{2}$ thus minimising potential systematic differences between $X_{d}$ and $X_{s}$ candidate efficiencies due to this cut.

Due to increased combinatoric backgrounds with larger values of $m_{X}$ the high mass region was previously observed to be statistically limited relative to the low mass resonant region despite it containing a larger fraction of the expected inclusive width [1]. Additionally modes where the hadronic component has a larger number of daughters or $\pi^{0}$ mesons will contribute more to combinatoric backgrounds. For this reason all cuts are optimised independently for six distinct classes in which both the upper mass limit and number of modes reconstructed in the high mass region are varied. Table 3.11 lists these classes and shows the total number events for the signal $B \rightarrow X_{d} \gamma \mathrm{MC}$ data with with at least one reconstructed $X_{d}$ candidate for each class before post reconstruction selections.

In the low mass resonant region there are three modes which dominate the signal contribution: 1,2 and 4 for $\rho^{0}, \rho^{+}$and $\omega$ transitions respectively. To investigate the background contributions from including further reconstructed modes in this region the cuts 


\begin{tabular}{|l|l|l|l|}
\hline Class & Mass Limit $\left(\mathrm{GeV} / c^{2}\right)$ & Included Modes & MC $X_{d}$ Evt $\geq 1$ (True) $X_{d}$ Cand \\
\hline 1 & 1.8 & $1,2,3,4$ & $202,696(75,347)$ \\
2 & 2.0 & $1,2,3,4$ & $231,639(84,816)$ \\
3 & 1.8 & $1,2,3,4,5,6,7$ & $371,307(98,582)$ \\
4 & 2.0 & $1,2,3,4,5,6,7$ & $432,987(112,752)$ \\
5 & 1.8 & $1,2,3,4,5,6,7,8,9$ & $429,513(110,592)$ \\
6 & 2.0 & $1,2,3,4,5,6,7,8,9$ & $490,169(126,743)$ \\
\hline
\end{tabular}

TABLE 3.11: Signal $B \rightarrow X_{d} \gamma \mathrm{MC}$ data in the high mass region with at least one $X_{d}$ candidate after applying cuts on the upper limit of reconstructed hadronic mass and number of reconstructed modes relevant to each optimisation class. Shown in parentheses are the number events with a correctly reconstructed candidate. The mode numbers correspond to those given in table 3.1

\begin{tabular}{|l|l|l|}
\hline Class & Included Modes & MC Evt $\geq 1$ (True) $X_{d}$ Cand: $\rho^{0} ; \rho^{+} ; \omega$ \\
\hline 7 & $1,2,4$ & 199,$690 ; 163,569 ; 74,625(190,712 ; 144,934 ; 38,480)$ \\
8 & $1,2,3,4,5,6,7$ & 202,$941 ; 171,091 ; 108,232(190,712 ; 144,934 ; 38,637)$ \\
\hline
\end{tabular}

TABle 3.12: Signal $B \rightarrow X_{d} \gamma \mathrm{MC}$ data in the low mass region with at least one $X_{d}$ candidate after applying cuts on reconstructed hadronic mass and number of reconstructed modes relevant to each optimisation class. Shown in parentheses are the number of events with a correctly reconstructed candidate.

are optimised independently for two classes which vary the number of reconstructed modes. Table 3.12 lists these classes again showing the number of events with at least one reconstructed $X_{d}$ candidate for signal MC data.

\subsubsection{Multivariate Classifier for Continuum Event Reduction}

The dominant contribution to combinatoric backgrounds is from continuum events. Ideally cuts on the topological and tagging variables discussed above can be used to reduce these backgrounds; however, the discrimination between signal and background for the majority of these variables is individually limited due to largely overlapping distributions. Therefore the use of a multivariate classifier is considered. Such classifiers use a number of user-defined input variables to create a discriminator which can recognise higher order patterns in data and use them to classify signal and background events. The classifier is trained on a subset of signal and background data where the data type is known beforehand. After training it can then be applied to further events to test them for consistency with the signal hypothesis. Such techniques are becoming increasingly common in high energy physics data analysis and in particular the BABAR collaboration have found them a particularly useful tool. In all tests neural networks (NN) are found to give the best performance for this analysis with MC data. Other multivariate classifiers investigated with these data include boosted and bagged decision trees; only 
the NN is discussed here. The following gives a general overview of NN discriminators, before describing the optimisation process implemented to find the best performing NN.

\subsubsection{Overview of Neural Networks}

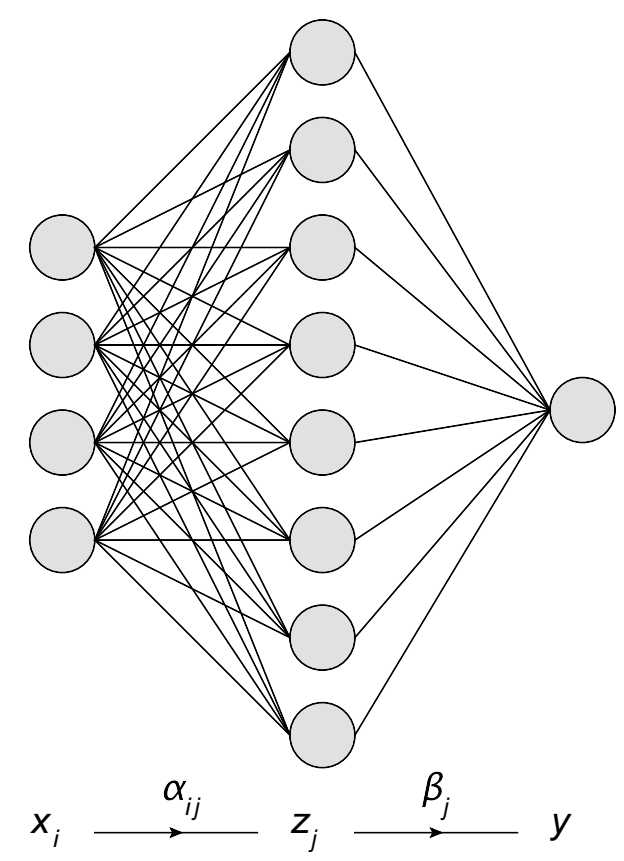

Figure 3.22: Schematic Representation of a Neural Network with four inputs, one hidden layer of eight nodes and one output.

Figure 3.22 shows a schematic representation of a typical NN with four input variables, one hidden layer with eight nodes and one output variable. The input variables, $x_{i}$, are normalised to the range $[0,1]$ and are related to each node, $j$, in the hidden layer by a weight $\alpha_{i j}$ as represented by the lines in figure 3.22. The hidden layer then has a value $z_{j}$ given by the output of an activation function, $\mathcal{A}$, whose input is the weighted sum of the input variables:

$$
z_{j}=\mathcal{A}_{j}\left(\sum_{i=1}^{4} \alpha_{i j} x_{i}\right) .
$$

Similarly the output, $y$, is given by,

$$
y=\mathcal{A}_{y}\left(\sum_{j=1}^{8} \beta_{j} z_{j}\right),
$$

where $\beta_{j}$ are weights between the hidden nodes and the output. Each training event has an associated quadratic classification error,

$$
\epsilon=(Y-y)^{2},
$$




\begin{tabular}{|l|l|}
\hline Variable & Cut Value \\
\hline$\pi^{0}$ veto upper limit & $\geq 0.15 \mathrm{GeV} / c^{2}$ \\
$\pi^{0}$ veto lower limit & $\leq 0.1 \mathrm{GeV} / c^{2}$ \\
$\eta$ veto upper limit & $\geq 0.59 \mathrm{GeV} / c^{2}$ \\
$\eta$ veto lower limit & $\leq 0.5 \mathrm{GeV} / c^{2}$ \\
$S_{\text {bump }}^{\prime}$ & $\leq 0.2$ \\
$x_{\text {trk }}$ & $\geq 25 \mathrm{~cm}$ \\
Lateral Moment & $\leq 0.6$ \\
$X_{d}\left|p_{\text {min }}\right|$ & $\geq 0.3 \mathrm{GeV} / c$ \\
$X_{d}$ vertex $\chi^{2}$ prob & $\geq 0.01$ \\
Pion PID Selector & pionKMTight \\
Use Endcap Photons & No \\
\hline
\end{tabular}

TABLE 3.13: Unoptimised cut values used to assign training events.

where $Y$ is the true event class, typically one for signal and zero for background. For each training event the network weights are systematically updated by propagating the previous quadratic error back from the output layer to the input layer. After all training events are exhausted one training cycle has been completed. An optimal NN will have $n$ training cycles where $n$ minimises the average quadratic error of a separate validation sample. This analysis uses the implementation of NN from the package StatPatternRecognition (SPR) [60], in which $\mathcal{A}$ is a sigmoid function.

\subsubsection{Classification of Training and Testing Events}

Training of the NN is carried out on continuum events which are as signal-like as possible. It is therefore desirable to apply all other post-reconstruction cuts beforehand. As the NN must be trained before cut optimisation a value for each cut to be optimised is chosen based on the experience of the previous BABAR analysis [1]. Table 3.13 lists the cut values chosen. After these cuts are applied the total efficiencies for simulated data are used to identify what proportion of these samples should be assigned to training to reflect the relative proportion of such events expected in data. Table 3.14 lists the required make up of training samples to reflect the expected proportion in data and indicates the proportion of events in each sample which must be assigned to training to obtain at least $10^{4}$ training events for each of the optimisation classes.

Training events are assigned such that they are evenly sampled across the MC data to ensure they accurately reflect varying background conditions from different running periods which are emulated in the simulation. A second mutually exclusive dataset sampled in the same way is used for evaluating the performance of NNs as they are 


\begin{tabular}{|l|l|l|}
\hline Data Samples & Train Sample & Fraction of MC data \\
\hline High Mass $X_{d}$ Signal $X_{d d} ; X_{d u}$ & $1: 1$ & $0.25 ; 0.25$ \\
High Mass $X_{d}$ Continuum uds; cc & $5: 1$ & $0.043 ; 0.041$ \\
Low Mass $X_{d}$ Signal $\rho^{0} ; \rho^{+} ; \omega$ & $4: 3: 1$ & $0.053 ; 0.059 ; 0.111$ \\
Low Mass $X_{d}$ Continuum uds; cc & $4: 1$ & $0.111 ; 0.111$ \\
\hline
\end{tabular}

TABLE 3.14: Required make up of training samples to reflect expected proportions in data and the required fraction of $\mathrm{MC}$ data needed to give greater than $10^{4}$ training events in each sample for each optimisation class.

\begin{tabular}{|l|l|l|}
\hline Class & Training Events (Sig; Cont) & Testing Events (Sig; Cont) \\
\hline \hline 1 & 11,$479 ; 11,977$ & 11,$412 ; 11,904$ \\
2 & 12,$984 ; 14,970$ & 12,$970 ; 14,898$ \\
3 & 13,$664 ; 21,813$ & 13,$615 ; 21,722$ \\
4 & 15,$624 ; 28,314$ & 15,$557 ; 28,205$ \\
5 & 15,$233 ; 29,462$ & 15,$162 ; 29,363$ \\
6 & 17,$485 ; 37,929$ & 17,$425 ; 37,832$ \\
\hline 7 & 15,$476 ; 14,333$ & 15,$421 ; 14,244$ \\
8 & 15,$478 ; 20,036$ & 15,$428 ; 19,928$ \\
\hline
\end{tabular}

TABLE 3.15: Size of NN training and testing samples for each optimisation class. There are two samples in each case, a sample of correctly reconstructed candidates and a sample of continuum backgrounds.

trained. For continuum events where there is more than one candidate in an event the candidate passing the requirements of best candidate selection described in section 3.6.6 is chosen.

For data analysis carried out after a NN cut has been applied, the use of events which trained the NN can lead to bias in efficiency calculations and fit studies. This is a consequence of potential over-training of the $\mathrm{NN}$ whereby the training dataset will give a better response than events not used for training. To eliminate this bias, training events are vetoed from the cut optimisation and subsequent studies; a correction factor is applied to account for this when calculating the efficiencies of signal event reconstruction. In signal MC data it is necessary to assign a consistent proportion of events which do not contain a correctly reconstructed candidate as training events even though they are not used in the actual training of the NN. This ensures that the correction applied to these data is the same for both correctly reconstructed candidates and combinatoric backgrounds; table 3.15 lists the size of test and training samples used for each optimisation class. 


\begin{tabular}{|l|l|}
\hline Variable Type & Considered Inputs \\
\hline Topological & $T_{R O E},\left|\cos \theta_{T}\right|, R_{2}^{\prime}, L_{2}^{T}, L_{3}^{T}, P_{1}^{T}, P_{2}^{T}, P_{3}^{T}, L_{1}^{\gamma}, L_{2}^{\gamma}, L_{3}^{\gamma}, P_{1}^{\gamma}, P_{2}^{\gamma}, P_{3}^{\gamma}$, \\
& $S_{R O E}, A_{R O E}, P_{R O E},\left|\cos \theta_{B}\right|$ \\
Tagging & $L_{T A G}^{e}, L_{T A G}^{\mu}, L_{T A G}^{l}, H_{T A G}^{\pi}, H_{T A G}^{K}, H_{T A G}^{K \pi}, P_{T A G}$ \\
\hline
\end{tabular}

TABLE 3.16: Variables considered for NN input.

\subsubsection{Training Strategy and Input Variable Selection}

There are 25 topological and tagging variables considered for the NN classifier which are listed in table 3.16. Note that from the definitions given in sections 3.3.2.1 and 3.3.2.2, $T_{R O E} \equiv L_{1}^{T}$, thus only $T_{R O E}$ is considered for NN input. Initially the structure of the $\mathrm{NN}$ is required to have one hidden layer with twice as many nodes as the input layer. For simplicity in implementing the analysis framework only one NN structure is optimised using the data corresponding to optimisation class 3 . The choice of these data is arbitrary, input variable distributions are not observed to change significantly between classes and so the choice of data used to train and test is not expected to significantly affect NN optimisation. Once the optimal NN is found it is then trained using each of the datasets listed in table 3.15, only the number of training cycles varies between optimisation classes.

When training a NN the training data are randomly split into two sub samples of equal size. One sample is used to train the NN and the other to calculate the average quadratic error every ten training cycles. Once the number of training cycles with minimum quadratic error is determined the $\mathrm{NN}$ is retrained with that many cycles using all training data and its performance evaluated. The performance evaluation of a given NN uses SPR tools with the testing data sample to calculate the percentage of background events accepted for a cut on the NN output which accepts 40, 30, 20 and 10\% of signal events. The calculated background acceptance for a given signal acceptance point has an associated statistical error. If the NN, when compared to an alternative NN, is found to have reduced background acceptance for one or more of the signal acceptance points the NN is considered to perform better. This is providing the improvement in background rejection percentage is greater than the calculated statistical error and that there is no significant degradation in background rejection at any of the other signal acceptance points.

SPR performs an internal calculation when training a NN, ranking the importance of the input variables. This calculation is not expected to be reliable but instead is used as a starting point when considering which variables to include. The NN is trained using all 26 input variables and the three highest ranked variables are then chosen to train the NN to be optimised. Variables are then added and removed in an arbitrary way to 
evaluate which combination gives the best performance. If the addition or removal of a variable results in a better performing $\mathrm{NN}$, determined by the criteria given above, then the resulting combination of input variables becomes the new baseline for adding and removing further variables. If no improvement in performance is observed then the preexisting combination of variables forms the baseline. If performance does not deteriorate on removing a variable then preference is given to the NN with fewer input variables. The optimum is found to have ten input variables: $T_{R O E}, R_{2}^{\prime}, L_{3}^{T}, L_{2}^{\gamma}, A_{R O E},\left|\cos \theta_{B}\right|$, $L_{T A G}^{e}, L_{T A G}^{\mu}, H_{T A G}^{\pi}$ and $H_{T A G}^{K}$. The number of hidden layers and corresponding nodes are again varied in an arbitrary way using these input variables. No NN is found to give better performance under these variations so the optimal NN is therefore determined to have one hidden layer with 20 nodes. The output of the optimal NN for both signal and continuum data not used for training is shown in figure 3.23 for event class three.

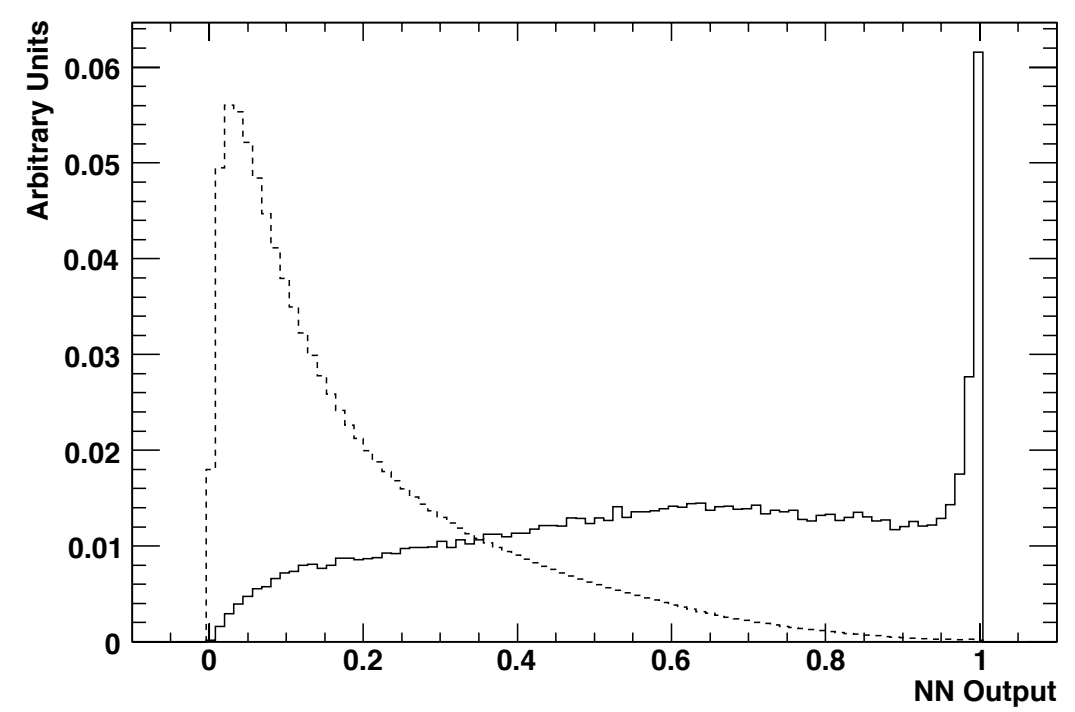

Figure 3.23: Normalised distributions of the NN output for continuum (dashed line) and correctly reconstructed candidates (solid line). These are MC data from event class three.

\subsubsection{Best Candidate Selection}

Once cuts have been applied there can still be events with more than one candidate. The likelihood fit described in chapter 4 assumes only one candidate per event so in the case of ambiguity a strategy for choosing which candidate to include in the fit is required. This selection must not bias the fit so the variables to be fitted, $\Delta E$ and $m_{E S}^{\prime}$, or any variable correlated with them cannot be used for candidate selection. 
There are two steps to choosing the best candidate. Firstly the best candidate within a given reconstructed mode is chosen. Secondly if an event has more than one reconstructed mode then a preferred mode must be selected. For the former the following criteria are applied. If a mode has a single neutral particle $\left(\pi^{0}\right.$ or $\left.\eta\right)$ then the candidate whose neutral daughter photon pair has invariant mass closest to the world average is chosen. For modes with two $\pi^{0}$ mesons this principle is extended into two dimensions by calculating the quantity,

$$
\Delta m_{\pi^{0}}=\sqrt{\left(m_{\pi^{0}}^{1}-m_{\pi^{0}}^{W A}\right)^{2}+\left(m_{\pi^{0}}^{2}-m_{\pi^{0}}^{W A}\right)^{2}},
$$

where $m_{\pi^{0}}^{i}$ is the invariant mass of the photon pair corresponding to the $i$ th $\pi^{0}$ and $m_{\pi^{0}}^{W A}$ is the world average $\pi^{0}$ mass. The candidate with $\Delta m_{\pi^{0}}$ closest to zero is chosen. If any ambiguity still exists or if a mode has no neutral daughters then the candidate with the highest vertex $\chi^{2}$ probability is chosen. For modes where ambiguity exists and there is only one charged daughter then the candidate whose track has the largest $\chi^{2}$ probability associated with its Kalman fit is chosen.

Investigations of signal MC data for patterns which may indicate an order of preference for choosing between candidates of different modes finds no preferred order so in the case of events where such ambiguity exists a random candidate is chosen.

\subsubsection{Cut Optimisation}

There are seven cuts for which simulated data are used to find an optimal value: the upper and lower limits of $\pi^{0}$ and $\eta$ invariant mass vetoes; the upper limit on $S_{\text {bump }}^{\prime}$; the lower limit on $\left|p_{\text {min }}\right|$; and the lower limit on the NN output. The choice of charged pion PID selector and use of endcap photons are also considered. Optimisation uses a simple cut and count technique in a signal region defined as $m_{E S}^{\prime}>5.27 \mathrm{GeV} / c^{2}$ and $-0.15 \leq \Delta E \leq 0.1 \mathrm{GeV}$. The figure of merit (FOM) is $s / \sqrt{b}$ where $s$ and $b$ are the estimated luminosity normalised contributions in the signal region of correctly reconstructed events and combinatoric backgrounds respectively. The normalisation of MC data to the experimental luminosity is discussed in section 3.7.1. This FOM is preferred to $s / \sqrt{s+b}$ to ensure it does not directly depend on correctly normalising the signal $B \rightarrow X_{d} \gamma \mathrm{MC}$ data; the contribution of incorrectly reconstructed $B \rightarrow X_{d} \gamma$ events to $b$ is small.

The optimisation is iterative. From a baseline of cuts, an individual iteration makes systematic variations in each cut; all other cuts are held constant and the cut under consideration is varied. Continuous variables are each varied over a range of 20 values, the start value and step size of which are shown in table 3.17. Ranges are chosen to 


\begin{tabular}{|l|l|l|}
\hline Cut & Start Value & Step \\
\hline upper $\pi^{0}$ veto & 0.110 & +0.005 \\
lower $\pi^{0}$ veto & 0.150 & -0.005 \\
upper $\eta$ veto & 0.530 & +0.005 \\
lower $\eta$ veto & 0.550 & -0.005 \\
$S_{\text {bump }}^{\prime}$ & 0.3 & -0.01 \\
$\left|p_{\text {min }}\right|$ & 0.300 & +0.025 \\
NN out & 0.60 & +0.02 \\
\hline
\end{tabular}

TABLE 3.17: Cut optimisation start point and step size for each continuous variable.

allow a significant proportion of phase space for each cut to be examined. For discrete variables the pion selector is varied to increasing levels of tightness and endcap photons are first considered and then vetoed. In each event, for each optimisation point, the best candidate selector is called if necessary then an event is tested for consistency with the signal region. The event is counted if it passes the cuts required for that optimisation point and is in the signal region. Correctly reconstructed candidate events contribute to $s$ while all potential background candidates contribute to $b$. Thus after normalising each MC data type to reflect the expected contribution in experimental data, a given optimisation point has an associated FOM. The value giving the best FOM for the cut under consideration is used as the baseline for the next iteration. In the first iteration the baseline corresponds to the cut values in table 3.13 and requires a candidate to have associated NN output greater than 0.8 .

The iterative process is continued until all cuts converge. If no convergence is observed after five iterations the set of cuts giving the best overall FOM is chosen. Table 3.18 lists the final FOM for each of the eight optimisation classes. The optimisation is performed independently for each event class.

This is a uni-directional optimisation process and is therefore only guaranteed to find a local maximum in the FOM; there may be an improved figure of merit found by changing the order in which variables are investigated. However, this analysis uses the cuts of the previous analysis [1] as a baseline which corresponds to a FOM of $\sim 1.5$ in the high mass region. These cuts were chosen using a similar FOM, optimised with an alternative method using independent MC data. Consequently as the optimisation here gives an improved result compared this baseline it is considered robust.

\subsubsection{Discussion of Analysis Strategy}

The cut optimisation results presented in table 3.18 show that in the high mass region class 2 , with four reconstructed modes and an upper hadronic mass limit of $2.0 \mathrm{GeV} / c^{2}$, 


\begin{tabular}{|l|l|l|l|}
\hline Class & $s$ & $b$ & $s / \sqrt{b}$ \\
\hline \hline 1 & 31.0 & 175.6 & 2.34 \\
2 & 25.1 & 109.4 & 2.40 \\
3 & 19.3 & 84.7 & 2.10 \\
4 & 31.2 & 232.4 & 2.05 \\
5 & 26.3 & 198.9 & 1.86 \\
6 & 21.0 & 129.3 & 1.85 \\
\hline 7 & 22.9 & 14.1 & 6.10 \\
8 & 38.3 & 61.4 & 4.89 \\
\hline
\end{tabular}

TABLE 3.18: Cut optimisation results for each optimisation class.

gives the most statistically significant measurement. In the low mass region the larger FOM is associated with class 7 which only reconstructs three modes. It follows that a measurement designed to maximise statistical significance should reconstruct fewer modes. However, following such a strategy leads to greater systematic uncertainty from model dependence when extrapolating to inclusive BFs. Clearly a balance must be struck between these competing factors and the decision of which optimisation class to use in each mass region must reflect this.

For the high mass region this analysis is required to be at least as inclusive as the previous $B A B A R$ measurement [1] which used optimisation class 3 ; this reconstructs seven modes with an upper hadronic mass limit of $1.8 \mathrm{GeV} / c^{2}$. The degradation in $s / \sqrt{b}$ between classes 3 and 4 is $<\sim 3 \%$ which is deemed to be an acceptable drop given the more inclusive nature of class 4 which reconstructs the same seven modes but over a larger hadronic mass range. However, moving to class 5 or 6 in lieu of class 3, i.e. including modes 8 and 9 which reconstruct two $\pi^{0}$ candidates, gives a decrease of $>\sim 10 \%$ in the statistical FOM. It is also noted that despite their more inclusive nature these classes have smaller $s$ due to cut optimisation points changing significantly ${ }^{5}$ as a result of increased combinatorics from modes 8 and 9 . In contrast class 4 has the largest value of $s$. Additionally modes 8 and 9 are susceptible to the $K_{S}^{0} \rightarrow \pi^{0} \pi^{0}$ background discussed in section 3.6.1. No specific reduction of this background is made and therefore, as such background is almost indistinguishable from signal, its presence can potentially bias the subsequent fit to data. The inclusion of modes 8 and 9 is therefore also undesirable for this reason. This analysis chooses optimisation class 4 in the high mass region. The upper hadronic mass limit is increased to $2.0 \mathrm{GeV} / c^{2}$ compared to $1.8 \mathrm{GeV} / c^{2}$ in the previous analysis; however, modes with two $\pi^{0}$ candidates in the hadronic reconstruction are not included in the measurement.

\footnotetext{
${ }^{5}$ Favouring tighter values.
} 


\begin{tabular}{|l|l|}
\hline Variable & Cut Value \\
\hline Hadronic mass upper limit & $\leq 2.0 \mathrm{GeV} / c^{2}$ \\
Included modes & $1,2,3,4,5,6,7$ \\
$m_{E S}$ & $\geq 5.22 \mathrm{GeV} / c^{2}$ \\
$\Delta E$ & $\leq 0.2 \mathrm{GeV}$ and $\geq-0.3 \mathrm{GeV}$ \\
$\pi^{0}$ veto & $\geq 0.155 \mathrm{GeV} / c^{2}$ and $\leq 0.095 \mathrm{GeV} / c^{2}$ \\
$\eta$ veto & $\geq 0.565 \mathrm{GeV} / c^{2}$ and $\leq 0.53 \mathrm{GeV} / c^{2}$ \\
$S_{\text {bump }}^{\prime}$ & $\leq 0.2$ \\
$x_{\text {trk }}$ & $\geq 25 \mathrm{~cm}$ \\
Lateral Moment & $\leq 0.6$ \\
$X_{d}\left|p_{\text {min }}\right|$ & $\geq 0.425 \mathrm{GeV} / c$ \\
$X_{d}$ vertex $\chi^{2}$ prob & $\geq 0.01$ \\
Pion PID Selector & pionKMTight \\
Use Endcap Photons & No \\
NN output & $\geq 0.86$ \\
\hline
\end{tabular}

TABLE 3.19: Optimised cut values in the high mass region for optimisation class 4 .

The low mass region is dominated by resonant decays and the model used to simulate it assumes no other contributions. This accounts for the $\sim 20 \%$ degradation in the FOM in moving from class 7 to class 8 which reconstructs four extra modes, none of which contribute to $s$ in a purely resonant model. If the low mass region is reconstructed as entirely resonant contributions without allowing for the other modes reconstructed in the high mass region then the assumptions of quark hadron duality may break down. In particular the feed-through of high mass contributions across the threshold boundary due to detector resolution is not accounted for as required in the resonance modified KN model [27]. This results in the theoretical uncertainty when extracting $\left|V_{t d} / V_{t s}\right|$ potentially being underestimated in chapter 1 . Therefore, although the low mass region is dominated by resonant transitions, its measurement should be as inclusive as possible so as not to invalidate the subsequent extraction of CKM matrix parameters. For this reason the analysis chooses class 8 in this region; the same seven modes reconstructed in the high mass region are also all reconstructed in the low mass region thus allowing any potential non-resonant contributions to be measured. Tables 3.19 and 3.20 show the final cut values determined for optimisation classes 4 and 8 respectively.

\subsection{Event Selection Summary}

Following the choice of analysis strategy and determination of optimum cut values the subsequent event selection efficiencies and projected yields in experimental data are 


\begin{tabular}{|l|l|}
\hline Variable & Cut Value \\
\hline Included modes & $1,2,3,4,5,6,7$ \\
$m_{E S}$ & $\geq 5.22 \mathrm{GeV} / c^{2}$ \\
$\Delta E$ & $\leq 0.2 \mathrm{GeV}$ and $\geq-0.3 \mathrm{GeV}$ \\
$\pi^{0}$ veto & $\geq 0.16 \mathrm{GeV} / c^{2}$ and $\leq 0.11 \mathrm{GeV} / c^{2}$ \\
$\eta$ veto & $\geq 0.56 \mathrm{GeV} / c^{2}$ and $\leq 0.52 \mathrm{GeV} / c^{2}$ \\
$S_{\text {bump }}^{\prime}$ & $\leq 0.19$ \\
$x_{\text {trk }}$ & $\geq 25 \mathrm{~cm}$ \\
Lateral Moment & $\leq 0.6$ \\
$X_{d}\left|p_{\text {min }}\right|$ & $\geq 0.6 \mathrm{GeV} / c$ \\
$X_{d}$ vertex $\chi^{2}$ prob & $\geq 0.01$ \\
Pion PID Selector & pionKMTight \\
Use Endcap Photons & No \\
NN output & $\geq 0.92$ \\
\hline
\end{tabular}

TABLE 3.20: Optimised cut values in the low mass region for optimisation class 8 .

presented. The final number of events with a reconstructed $B \rightarrow X_{s / d} \gamma$ candidate in MC data after vetoing both NN training events and any $B \rightarrow X_{s} \gamma$ events in generic $B \mathrm{MC}$ data are shown in table 3.21. The total number of $B \rightarrow X_{d} \gamma$ candidate events from $B \rightarrow X_{s} \gamma \mathrm{MC}$ data with an intermediate $K_{S}^{0} \rightarrow \pi^{+} \pi^{-}$decay which are correctly reconstructed are 1 and 6 in the high and low mass regions respectively. This shows that the selections applied in section 3.6.1 make such backgrounds negligible.

\subsubsection{Estimated Data Yields and Selection Efficiencies}

Table 3.21 can be used to estimate the expected yields for each sample in the experimental data. Estimated yields are calculated from the total number of $B \bar{B}$ pairs in experimental data (given in section 3.1.1) using the assumed BFs for signal modes and the calculated cross sections for $e^{+} e^{-} \rightarrow q \bar{q}(q=u, d, s, c)$ transitions at the $\Upsilon(4 S)$ mass [4]. Correction factors to convert between MC data yields and estimated experimental data yields are calculated by taking the ratio of the expected data yield with the total MC dataset sizes listed in tables 3.3 and 3.4 for signal and table 3.2 for generic backgrounds. Signal MC data requires a further factor of two correction to account for the forced decay of one $B$ meson in an event to a signal mode. In all calculations it is assumed that the transitions $\Upsilon(4 S) \rightarrow B^{+} B^{-}$and $\Upsilon(4 S) \rightarrow B^{0} \bar{B}^{0}$ are equally likely. This assumption is consistent with current experimental measurements of $\Upsilon(4 S) \rightarrow B \bar{B}$ transitions [14]; however, it means the uncertainty associated with such measurements is not considered a source of systematic uncertainty. 


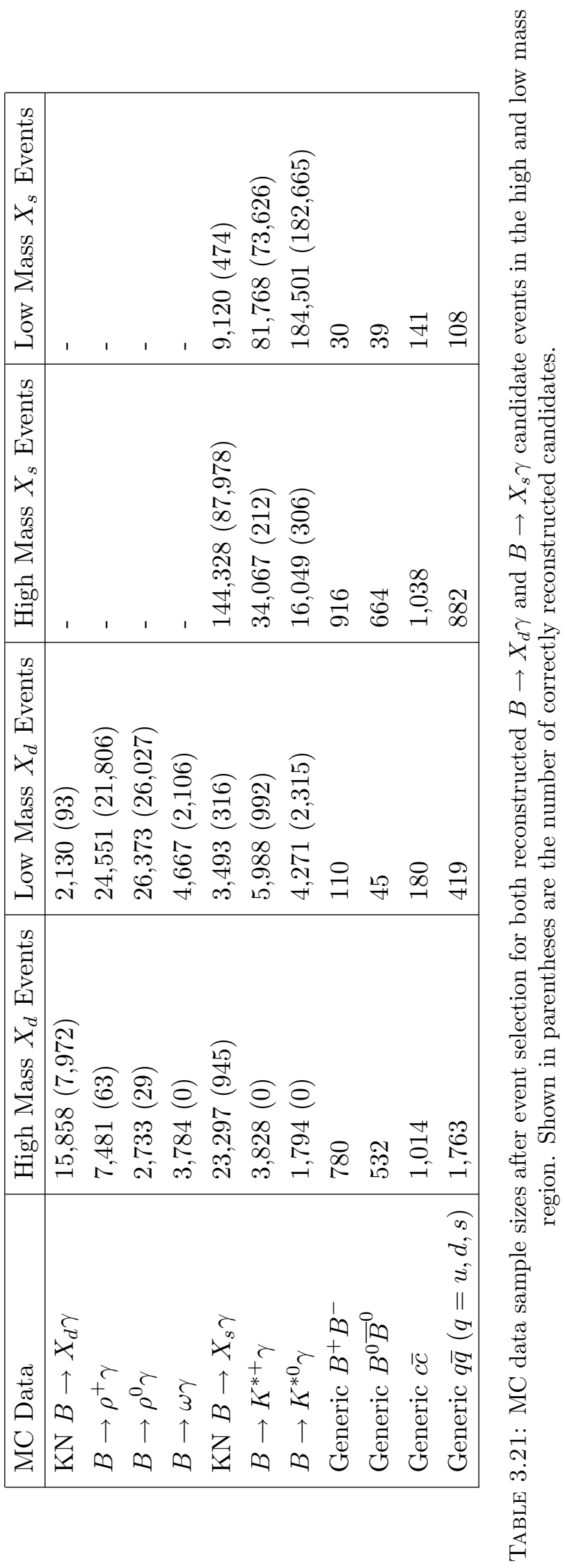




\begin{tabular}{|c|c|}
\hline & 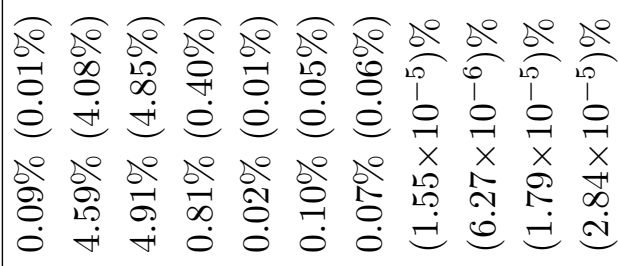 \\
\hline & 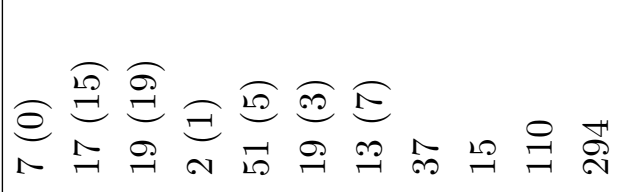 \\
\hline & 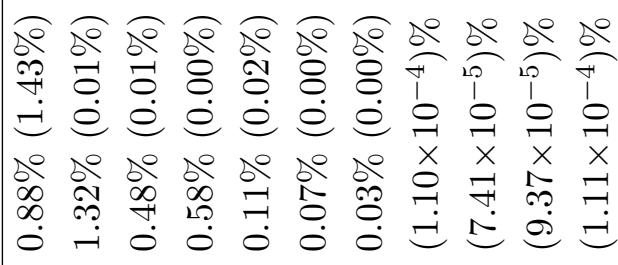 \\
\hline & 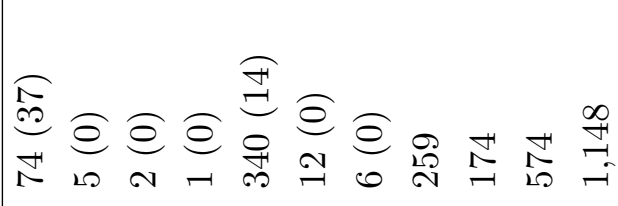 \\
\hline & 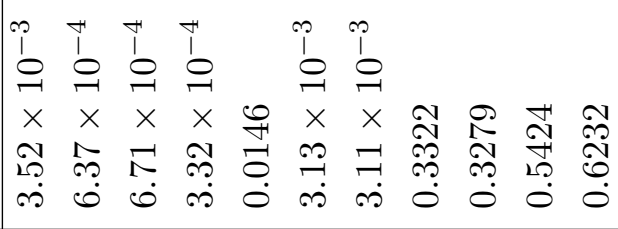 \\
\hline$\vec{E}$ & 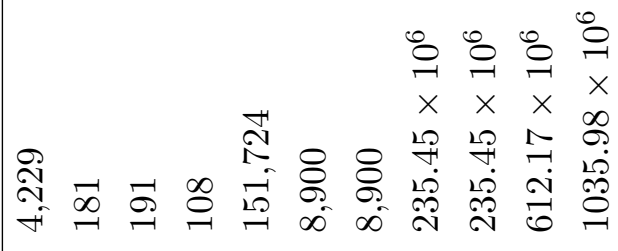 \\
\hline $\begin{array}{l}+1 \\
\infty \\
\infty \\
0\end{array}$ & 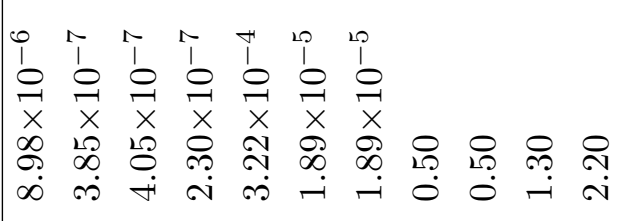 \\
\hline & 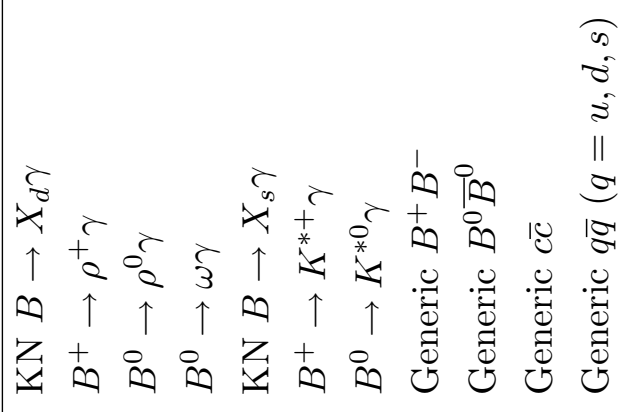 \\
\hline
\end{tabular}

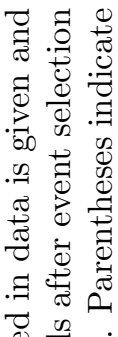

过

罂

要要

:

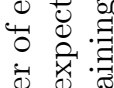

突

夏至

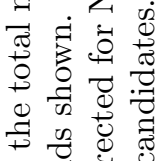

$+0$

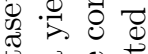

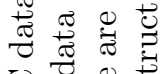

닐

가월

ब.

玒

D.

承 $\frac{9}{3}$

兽要

范皆

苛

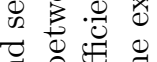

का

을

5.8

证

要

ซี

․ㅜㅇ

范

.9007

되 :응

ชี

ते है

되당

焉要 


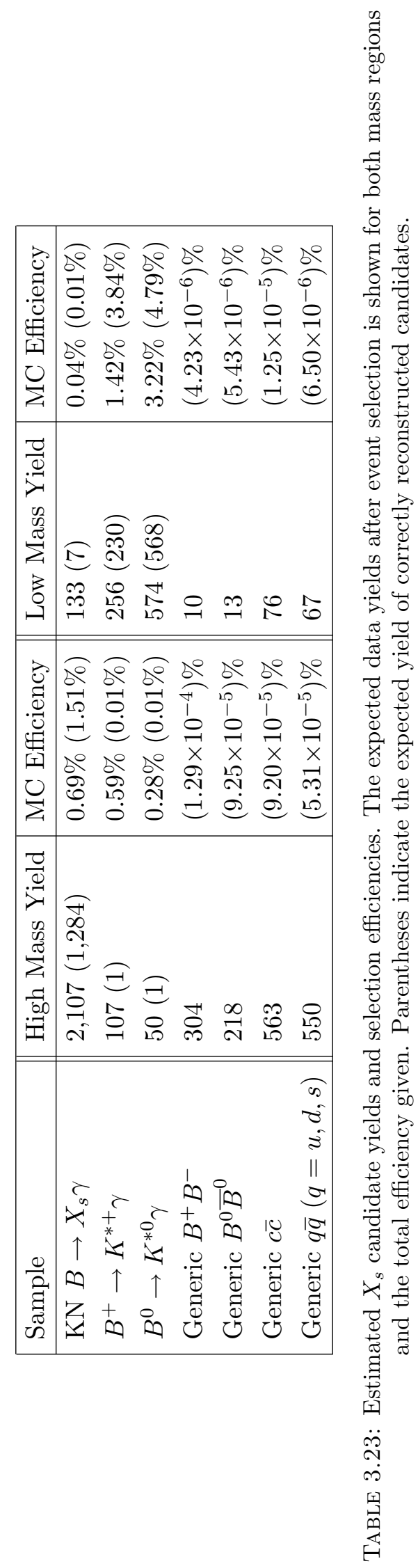




\begin{tabular}{|l||l|l||l|l|}
\hline Mode & $X_{d}$ Events & Efficiency & $X_{s}$ Events & Efficiency \\
\hline 1 & 2,570 & $3.45 \%$ & 34,580 & $3.56 \%$ \\
2 & 2,204 & $2.65 \%$ & 15,486 & $2.60 \%$ \\
3 & 1,185 & $1.65 \%$ & 19,802 & $1.80 \%$ \\
4 & 1,198 & $0.81 \%$ & 10,695 & $0.80 \%$ \\
5 & 156 & $0.47 \%$ & 1,784 & $0.45 \%$ \\
6 & 398 & $0.29 \%$ & 2,968 & $0.30 \%$ \\
7 & 261 & $2.72 \%$ & 2,663 & $3.13 \%$ \\
\hline
\end{tabular}

TABLE 3.24: Generated events and total efficiency by mode for correctly reconstructed high mass $X_{d}$ and $X_{s}$ candidates.

\begin{tabular}{|l||ll|l||ll|l|}
\hline $\begin{array}{l}\text { Mass } \\
\left(\mathrm{GeV} / c^{2}\right)\end{array}$ & $\begin{array}{l}X_{d} \text { Events } \\
\text { Generated }\end{array}$ & Post-Cuts & Efficiency & $X_{s}$ Events & Efficiency \\
\hline $1.0-1.2$ & 134,444 & 1,901 & $1.89 \%$ & 802,481 & 19,511 & $2.43 \%$ \\
$1.2-1.4$ & 149,463 & 2,012 & $1.79 \%$ & $1,051,158$ & 23,404 & $2.23 \%$ \\
$1.4-1.6$ & 136,874 & 1,694 & $1.65 \%$ & $1,052,711$ & 19,690 & $1.87 \%$ \\
$1.6-1.8$ & 110,729 & 1,363 & $1.64 \%$ & 885,260 & 14,956 & $1.69 \%$ \\
$1.8-2.0$ & 80,139 & 958 & $1.59 \%$ & 652,094 & 10,072 & $1.54 \%$ \\
$>2.0$ & 131,611 & 44 & $0.04 \%$ & $1,039,348$ & 345 & $0.03 \%$ \\
\hline
\end{tabular}

TABLE 3.25: Generated signal modes and total efficiency as a function of generated hadronic mass for correctly reconstructed high mass $X_{d}$ and $X_{s}$ candidates.

Table 3.22 shows the estimated experimental data yields for all data samples before event selection. The correction factors are presented and post-selection yields for $B \rightarrow X_{d} \gamma$ candidate events are calculated applying the correction factors to the MC data sample sizes listed in 3.21. Where necessary further corrections have been made to account for vetoed NN training events. Also shown are the overall efficiency calculations of the event selection on each MC sample ${ }^{6}$. The corresponding post selection estimated yields and efficiencies for $B \rightarrow X_{s} \gamma$ candidate events are presented in table 3.23. A detailed breakdown of the number of generated events in signal MC data corresponding to a reconstructed mode are presented in appendix B. These generated numbers are used to calculate mode by mode efficiencies which are presented in table 3.24 for both flavours of data in the high mass bin.

Finally table 3.25 shows the selection efficiency for correctly reconstructed $B \rightarrow X_{d} \gamma$ and $B \rightarrow X_{s} \gamma$ candidate events as a function of generated hadronic mass in the high mass region. The denominator in this efficiency ratio corresponds to all generated signal

\footnotetext{
${ }^{6}$ The signal efficiencies here do not necessarily directly correspond to those given in section 4.6.4 as the respective denominators in efficiencies used to calculate measured $\mathrm{BF}$ values only consider events with generated hadronic mass within the reconstructed mass range
} 
modes in the corresponding bin of generated hadronic mass before event selections are applied. 


\section{Chapter 4}

\section{Signal Yield Extraction}

Yields for the number of correctly reconstructed candidates in experimental data, after event selection, are extracted using a two dimensional (2D) unbinned extended maximum likelihood fit ${ }^{1}$ to the kinematic variables $\Delta E$ and $m_{E S}^{\prime}$. The fit strategy is optimised using simulated data samples. This chapter introduces the maximum likelihood fit (MLF) technique, before discussing the probability density functions (PDFs) used to model different event hypotheses and their parameterisation using MC data. The combined fitting strategy to be used on experimental data is outlined and studies for bias in this strategy using emulated and embedded MC data samples are presented. Finally the results of each of the four fits to the experimental data are given.

\subsection{Maximum Likelihood}

The MLF is a powerful statistical tool described in detail elsewhere [63, 64], an overview of which is given here. It can be advantageous compared with other fitting techniques, such as least squares, for it treats data on an event by event basis thus avoiding bias which can arise from binning samples beforehand.

\subsubsection{Overview}

Consider a sample of $N$ events described by variable $x$ whose parent distribution is a normalised PDF, $\mathcal{P}\left(x ; \alpha_{1}, . ., \alpha_{n}\right)$, described by $n$ parameters, $\alpha_{k}(k=1, . ., n)$. The sample can provide estimators for each parameter, $\hat{\alpha}_{k}$, through the principle of maximum likelihood (PML). For a given set of estimators the likelihood, $\mathcal{L}$, for the sample is given

\footnotetext{
${ }^{1}$ All computational fits use the RooFit [61] toolkit which implements the Minuit core package [62].
} 
by,

$$
\mathcal{L}\left(\hat{\alpha}_{1}, . ., \hat{\alpha}_{n}\right)=\prod_{i=1}^{N} \mathcal{P}\left(x_{i} ; \hat{\alpha}_{1}, . ., \hat{\alpha}_{n}\right) .
$$

This represents the joint probability density of obtaining the $x_{i}$ values observed $^{2}$. The PML states that the set of $\hat{\alpha}_{k}$ which maximise $\mathcal{L}$ will best describe the data as these give a higher cumulative probability. Therefore iterative variation of estimators to find the maximum value of $\mathcal{L}$ provides a robust method of estimating the parameterisation of $\mathcal{P}\left(x ; \alpha_{1}, . ., \alpha_{n}\right)$, thus fitting the PDF to the data sample.

It is possible to generalise (4.1) such that a sample described by two independent variables, $(x, y)$, and constructed from $M$ event hypotheses, e.g. signal and different backgrounds, will have a likelihood function given by,

$$
\mathcal{L}\left(\hat{\alpha}_{1}^{x}, . ., \hat{\alpha}_{n}^{x}, \hat{\alpha}_{1}^{y}, . ., \hat{\alpha}_{m}^{y}\right)=\prod_{i=1}^{N}\left[\sum_{j=1}^{M} w_{j} \mathcal{P}_{j}^{x}\left(x_{i} ; \hat{\alpha}_{1}^{x}, . ., \hat{\alpha}_{n}^{x}\right) \mathcal{P}_{j}^{y}\left(y_{i} ; \hat{\alpha}_{1}^{y}, . ., \hat{\alpha}_{m}^{y}\right)\right]
$$

where $w_{j}$ is the assigned weight of each hypothesis.

For computational calculations it is more convenient to find the best set of estimators by minimising the negative log-likelihood, $l$, as the product over $N$ events in (4.1) becomes a sum,

$$
l=-\ln \mathcal{L}=-\sum_{i=1}^{N} \ln \mathcal{P}\left(x_{i} ; \hat{\alpha}_{1}, . ., \hat{\alpha}_{n}\right) .
$$

The best values for each estimator are obtained from the set of simultaneous equations,

$$
\frac{\partial l}{\partial \hat{\alpha}_{k}}=0
$$

Corresponding uncertainties on estimator values are calculated by taking second derivatives of $l$, assuming the minimum is locally parabolic. The associated error matrix, $E$, is given by,

$$
\left(E^{-1}\right)_{i j}=\left(\frac{\partial^{2} l}{\partial \hat{\alpha}_{i} \partial \hat{\alpha}_{j}}\right)
$$

\subsubsection{Extended Maximum Likelihood}

The above discussion concerns estimators calculated to determine the shape of data distributions where the PDFs are normalised such that,

$$
\int \mathcal{P}\left(x ; \alpha_{1}, . ., \alpha_{n}\right) d x=1 .
$$

\footnotetext{
${ }^{2}$ Omitted from the right hand side of (4.1) and subsequent equations are factors of $1 / N$ ! as ultimately it is variations in $\mathcal{L}$ which are of interest.
} 
However, if the normalisation is also an unknown to be determined from the fit, the extended maximum likelihood fit (EMLF) is required. This method relaxes the constraint of (4.6) replacing $\mathcal{P}\left(x ; \alpha_{1}, . ., \alpha_{n}\right)$ with a function $\mathcal{Q}\left(x ; \alpha_{1}, . ., \alpha_{n}\right)$ such that,

$$
\int \mathcal{Q}\left(x ; \alpha_{1}, . ., \alpha_{n}\right) d x=\nu,
$$

where $\nu$ is the mean sample size. In finite data where events are sampled at random the actual sample size $N$ will not necessarily coincide with $\nu$. This is reflected in the EMLF by multiplying the likelihood by the Poisson probability of obtaining $N$ events given a mean $\nu$ such that,

$$
\begin{aligned}
l & =-\ln \left(e^{-\nu} \nu^{N}\right)-\sum_{i=1}^{N} \ln \mathcal{P}\left(x ; \alpha_{1}, . ., \alpha_{n}\right), \\
& =\nu-\sum_{i=1}^{N} \ln \mathcal{Q}\left(x ; \alpha_{1}, . ., \alpha_{n}\right) .
\end{aligned}
$$

As the normalisation of $\mathcal{Q}$ increases, the sum on the right hand side of (4.8) will become more negative; however, the opposite is true of the $\nu$ term. Thus, by balancing these effects, minimising $l$ will find the most probable value of $\nu$ given the PDF parameterisation of $x$ for a sample of $N$ events.

\subsubsection{Fit Variables}

The choice of $m_{E S}^{\prime}$ and $\Delta E$ as fit variables aims to maximise statistical significance when fitting to experimental data. The use of two variables improves the ability of the fit to discriminate between different event hypotheses. Where possible event hypotheses are modelled as the product of uncorrelated PDFs following the formalism of (4.2). Consequently the variables used in the fit should be essentially independent. The variables $m_{E S}$ and $\Delta E$ are to a good approximation independent for signal; however, as a consequence of (3.3) this is not necessarily true for $m_{E S}^{\prime}$ and $\Delta E$. Despite this, previous $B A B A R$ analyses in the radiative penguin decay working group have shown that $m_{E S}^{\prime}$ and $\Delta E$ are suitable variables for a two dimensional MLF $[1,30,59]$. The improved resolution obtained from $m_{E S}^{\prime}$ and the need to be consistent with previous BABAR radiative penguin analyses therefore makes it the preferential variable to fit for over $m_{E S}$.

\subsection{Corrections to $B \rightarrow X \gamma \mathrm{MC}$ data}

Previous measurements with $B A B A R$ experimental data of $B \rightarrow X_{s} \gamma$ transitions show that JETSET phase space decays do not accurately model the relative contributions 


\begin{tabular}{|l|l|l|l|}
\hline Fit & Yield & Pre-weighting & Post-weighting \\
\hline$B \rightarrow X_{s}^{L} \gamma$ & Signal & 805 & 801 \\
& Mass Cross-feed & 126 & 162 \\
\hline$B \rightarrow X_{s}^{H} \gamma$ & Signal & 1284 & 1112 \\
& Self Cross-feed & 823 & 879 \\
\hline \multirow{2}{*}{$B \rightarrow X_{d}^{L} \gamma$} & PID Cross-feed & 15 & 13 \\
& $X_{s}$ Mass Cross-feed & 46 & 36 \\
\hline \multirow{2}{*}{$B \rightarrow X_{d}^{H} \gamma$} & PID Cross-feed & 14 & 11 \\
& $X_{s}$ Self Cross-feed & 326 & 324 \\
\hline
\end{tabular}

TABLE 4.1: Corrected event yield estimates for $B \rightarrow X_{s} \gamma$ event hypotheses after MC data weighting.

from different final states $[1,30]$. These analyses calculated weights (assumed to be independent of hadronic mass) which can be applied to different classes of MC events to make the model more representative of the experimental data. Corrections are not applied to resonant signal MC as $K^{*}, \rho$ and $\omega$ decays are well measured [14].

The analysis in [30] reconstructed 38 exclusive $B \rightarrow X_{s} \gamma$ final states in the hadronic mass range $1.1 \leq m_{X} \leq 2.8 \mathrm{GeV} / c^{2}$ using experimental data up to and including Run 2. For the purposes of weighting MC data these final states are classified into ten distinct sets. The analysis in [1] is the previous version of the analysis presented here and reconstructed modes 1-7 of table 3.1 in the hadronic mass range $1.0 \leq m_{X} \leq$ $1.8 \mathrm{GeV} / c^{2}$ using experimental data up to and including Run 5. Summaries of final state contributions in generated $B \rightarrow X \gamma \mathrm{MC}$ data are given in appendix $\mathrm{B}$ and the application of weights to $B \rightarrow X_{s} \gamma \mathrm{MC}$ events is summarised in table B.6. Weights are applied such that the overall normalisation of MC data before cuts is constant resulting in a further correction weight to any mode not reconstructed in either of the above analyses to preserve unity. After weights have been applied the change in the relative contribution of individual events means the post event selection $B \rightarrow X_{s} \gamma$ MC data yields and thus expected experimental data yields must be corrected. Table 4.1 shows the corrected estimated experimental data yields. These are compared to the unweighted estimates given in tables 3.22 and 3.23. All subsequent plots and calculations pertaining to $B \rightarrow X_{s} \gamma \mathrm{MC}$ events in the high mass region use the weighted data.

No correction is made to $B \rightarrow X_{d} \gamma$ data generated through the JETSET phase space model as information from experimental data does not exist; a naive application of the $B \rightarrow X_{s} \gamma$ weights to $B \rightarrow X_{d} \gamma \mathrm{MC}$ events is performed in section 5.1.4.2 as part of the study of systematic uncertainties in this analysis. 


\subsection{Event Hypotheses}

In fits to experimental data all potential event hypotheses must be identified and their relative contribution modelled. Here contributing events are determined from MC data. The respective high and low mass regions ${ }^{3}$ for a given flavour of $B \rightarrow X \gamma$ candidates are modelled with identical event hypotheses. The following section presents all of the event hypotheses identified for this analysis.

\subsection{1 $\quad B \rightarrow X_{s} \gamma$ Candidate Events}

Five contributing event hypotheses are identified for $B \rightarrow X_{s} \gamma$ candidates passing all event selection cuts. Signal MC data can contribute either correctly or incorrectly reconstructed events. Incorrectly reconstructed signal events are divided into those where the generated decay corresponds to the reconstructed mass region ${ }^{4}$ (self cross-feed) and those where the generated decay was from the other mass region (mass cross-feed). The remaining two event hypotheses are any other $B \bar{B}$ event (generic $B \bar{B}$ ) and any continuum event. Figure 4.1 shows $2 \mathrm{D}$ plots of $m_{E S}^{\prime}$ and $\Delta E$ from $\mathrm{MC}$ data in the low mass region. The corresponding plots for the high mass region are shown in figure 4.2. Signal, generic $B \bar{B}$ and continuum event distributions can be sufficiently fitted as the product of independent PDFs for each variable. The same is not true for cross-feed events where the combination of different background contributions leads to correlations between the fit variables. For example signal decays with a $\pi^{0}$ in the final state have enhanced backgrounds in the signal region due to background candidates where a relatively low energy photon from the $\pi^{0}$ decay is exchanged for a similar background photon. The resulting candidate will be kinematically very similar to the generated signal candidate; this is particularly evident in low mass $B^{+} \rightarrow K^{*+} \gamma$ self cross-feed events where the hadronic state is reconstructed from $K^{*+} \rightarrow K^{+} \pi^{0}$ decays. Such background does not exist in decays without a neutral hadronic final state daughter. Other contributions arise from backgrounds where the reconstructed candidate was not the same as the generated signal candidate or where the generated event was not a signal decay. In theory such correlations in these data can be more accurately modelled by identifying each background category and assigning it a separate event hypothesis; however, this is impractical as there are many such categories and the resulting combined fit to experimental data would become unmanageable. Consequently cross-feed distributions must be modelled as 2D histogram PDFs whose shapes are determined from MC data.

\footnotetext{
${ }^{3}$ To simplify notation, $X^{L}$ will be used to indicate a hadronic candidate with reconstructed mass in the range $0.5 \leq m_{X}<1.0 \mathrm{GeV} / c^{2}$ and $X^{H}$ a hadronic candidate with reconstructed mass in the range $1.0 \leq m_{X} \leq 2.0 \mathrm{GeV} / c^{2}$

${ }^{4}$ Any cross-feed component from the high mass bin includes events where the generated hadronic mass was greater than $2.0 \mathrm{GeV} / c^{2}$
} 
The choice of binning for these histograms is arbitrary and depends on the quantity of MC data available; more data allow finer binning. In this analysis the statistics in all cross-feed MC data are sufficient to allow ten bins in each of the signal regions defined in the previous chapter for cut optimisation. This results in 20 bins for $\Delta E$ and 35 bins for $m_{E S}^{\prime}$ across the full fit range.

\subsection{2 $B \rightarrow X_{d} \gamma$ Candidate Events}

Eight event hypotheses are identified for $B \rightarrow X_{d} \gamma$ candidates passing all event selection cuts. Five of these directly correspond to the event hypotheses in $B \rightarrow X_{s} \gamma$ data: Correctly reconstructed events, self cross-feed events, mass cross-feed events, generic $B \bar{B}$ events and continuum events. The additional three event hypotheses correspond to cross-feed contributions where $B \rightarrow X_{d} \gamma$ candidates were reconstructed in $B \rightarrow X_{s} \gamma$ data. The three categories are correctly reconstructed $B \rightarrow X_{s} \gamma$ events where the generated charged kaon was reconstructed as a charged pion due to PID inefficiency (PID cross-feed) and incorrectly reconstructed events (separated into $X_{s}$ self cross-feed and $X_{s}$ mass cross-feed analogous to the signal cross-feed contributions). Figure 4.3 shows $2 \mathrm{D}$ plots of $m_{E S}^{\prime}$ and $\Delta E$ from $\mathrm{MC}$ data in the low mass region. The corresponding plots for the high mass region are shown in figure 4.4. Signal, PID cross-feed, generic $B \bar{B}$ and continuum event distributions can be sufficiently fitted as the product of independent PDFs in each variable. The remaining cross-feed events are again correlated due to combinations of different background contributions and must therefore be modelled as 2D histogram PDFs.

\subsection{PDF Parameterisation}

The choice of PDF parameterisation for different event hypotheses is determined from MLFs to MC data passing the event selection criteria. A set of PDFs is required for each of the four fits to be carried out. Presented here are the sets of PDFs which were found to best fit the MC data and their fitted parameters from those data.

\subsubsection{PDF Functional Forms}

The functional form for all PDF distributions used in the subsequent MLFs are given here for a general variable $x$ and normalisation factor $\mathcal{N}$. Each parameter to be fitted is introduced. 


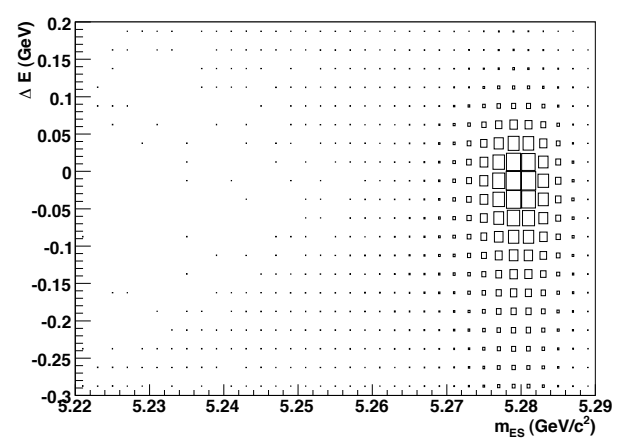

(a) $B \rightarrow K^{*} \gamma$ signal events with a correctly reconstructed candidate (signal).

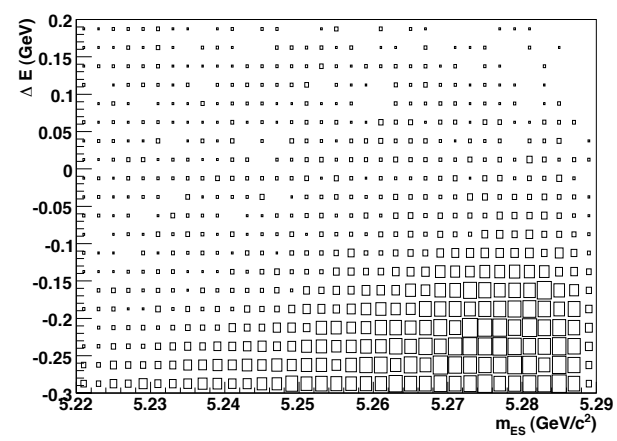

(c) $B \rightarrow X_{s} \gamma \mathrm{KN}$ model events where the candidate was incorrectly reconstructed (mass cross-feed).

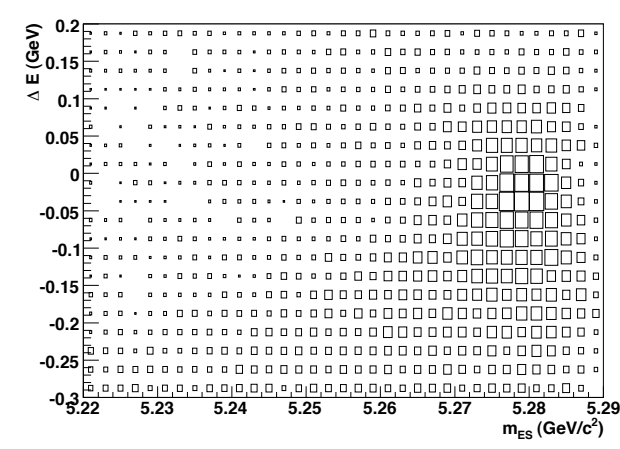

(b) $B \rightarrow K^{*} \gamma$ events where the candidate was incorrectly reconstructed (self cross-feed).

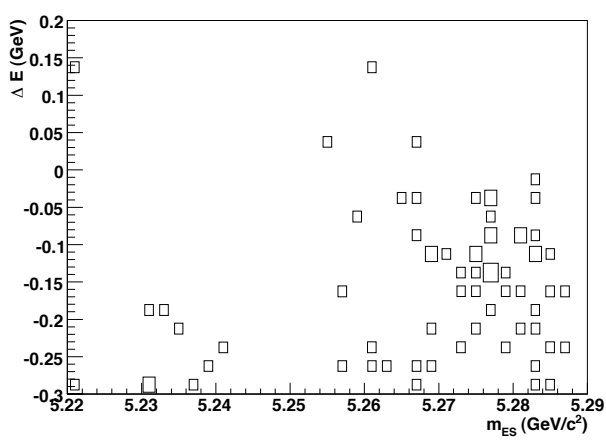

(d) Generic $B \bar{B}$ events.

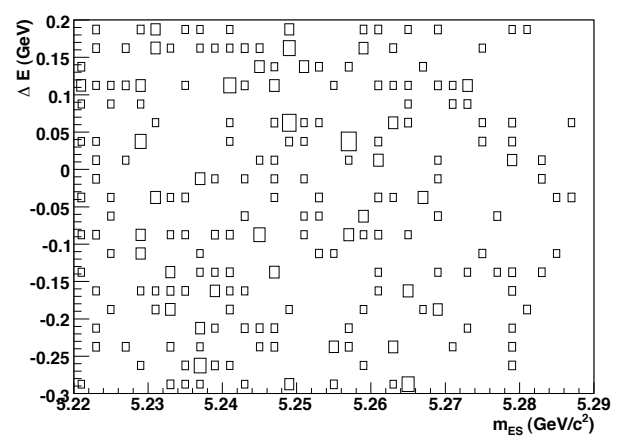

(e) Continuum events.

Figure 4.1: 2D plots of $m_{E S}^{\prime}$ and $\Delta E$ showing $B \rightarrow X_{s}^{L} \gamma$ candidates in each event hypothesis for MC data passing event selection cuts. The number of events in each plot reflects the MC data statistics given in table 3.21. Individual components in the generic $B \bar{B}$ and continuum data are weighted such that the combined data plots reflect any difference in relative contributions expected from those components in experimental data. 


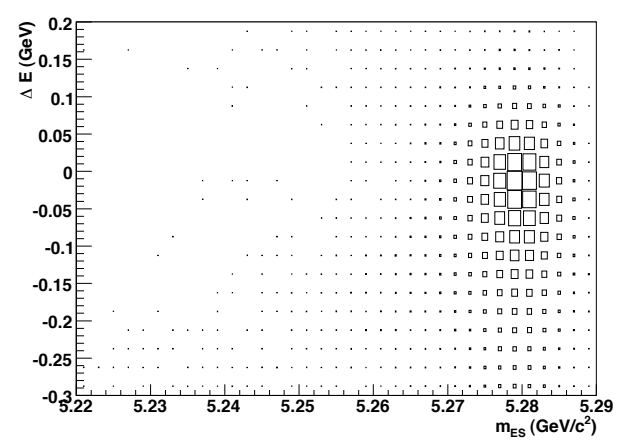

(a) $B \rightarrow X_{s} \gamma \mathrm{KN}$ model events where the candidate was correctly reconstructed (signal).

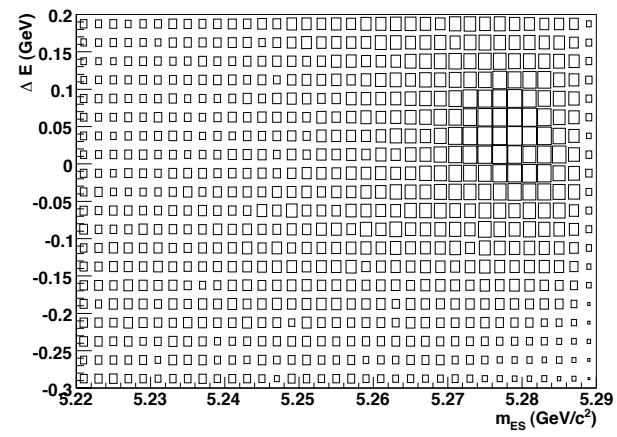

(c) $B \rightarrow K^{*} \gamma$ events where the candidate was incorrectly reconstructed (mass cross-feed).

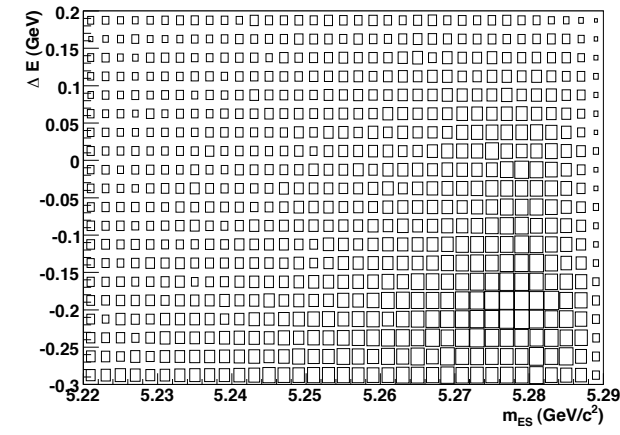

(b) $B \rightarrow X_{s} \gamma \mathrm{KN}$ model events where the candidate was incorrectly reconstructed (self cross-feed).

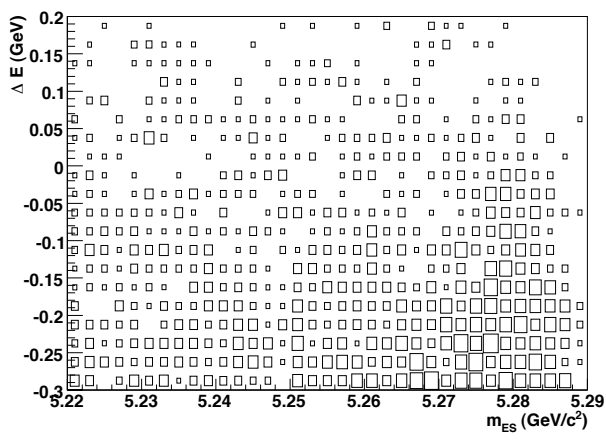

(d) Generic $B \bar{B}$ events.

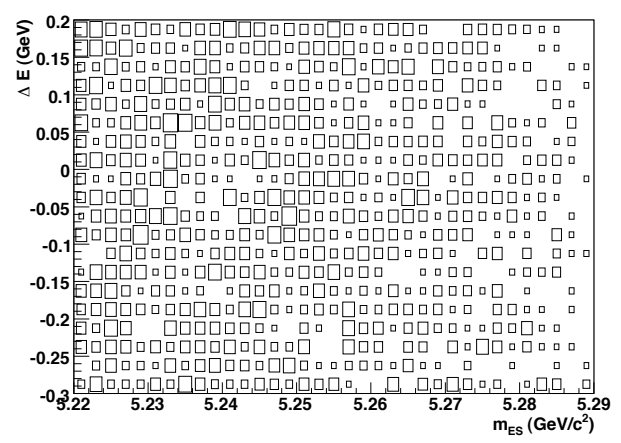

(e) Continuum events.

Figure 4.2: 2D plots of $m_{E S}^{\prime}$ and $\Delta E$ showing $B \rightarrow X_{s}^{H} \gamma$ candidates in each event hypothesis for MC data passing event selection cuts. The number of events in each plot reflects the MC data statistics given in table 3.21. Individual components in the generic $B \bar{B}$ and continuum data are weighted such that the combined data plots reflect any difference in relative contributions expected from those components in experimental data. 


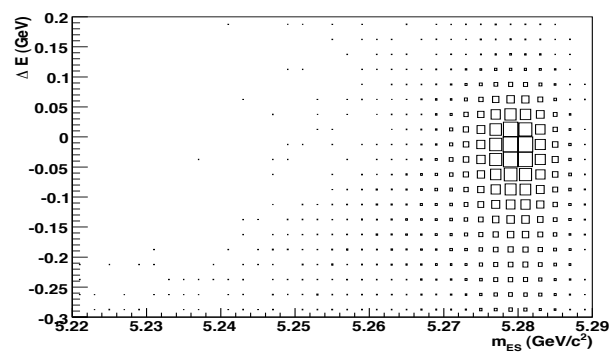

(a) $B \rightarrow \rho / \omega \gamma$ events where the candidate was correctly reconstructed (signal).

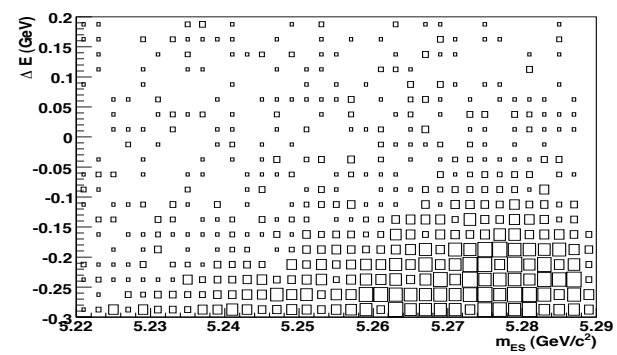

(c) $B \rightarrow X_{d} \gamma$ events where the candidate was incorrectly reconstructed (mass cross-feed).

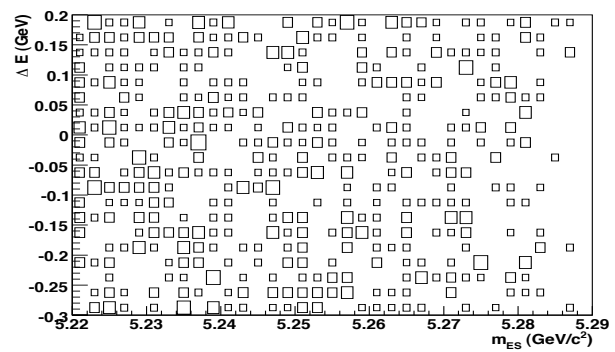

(e) Continuum events.

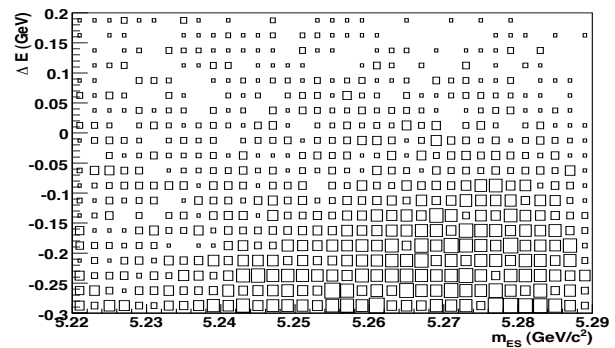

(g) $B \rightarrow X_{s} \gamma X_{s} \mathrm{KN}$ model events where the candidate was incorrectly reconstructed $\left(X_{s}\right.$ mass crossfeed).

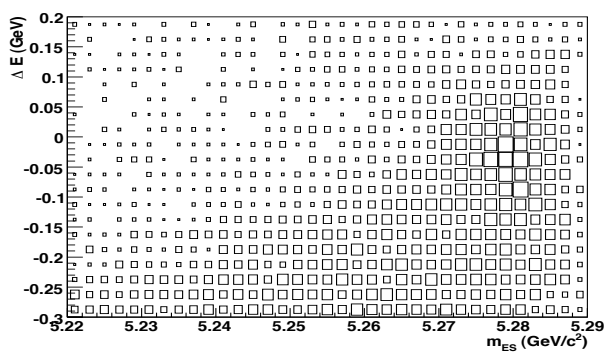

(b) $B \rightarrow \rho / \omega \gamma$ events where the candidate was incorrectly reconstructed (self cross-feed).

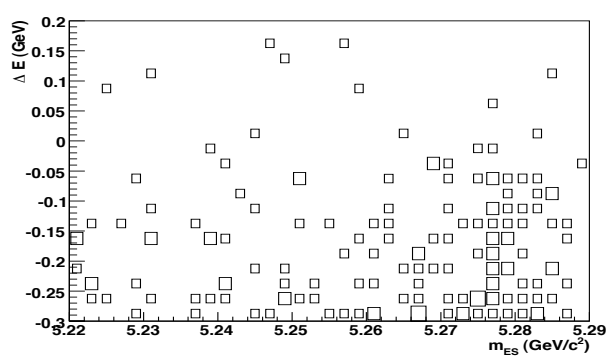

(d) Generic $B \bar{B}$ events.

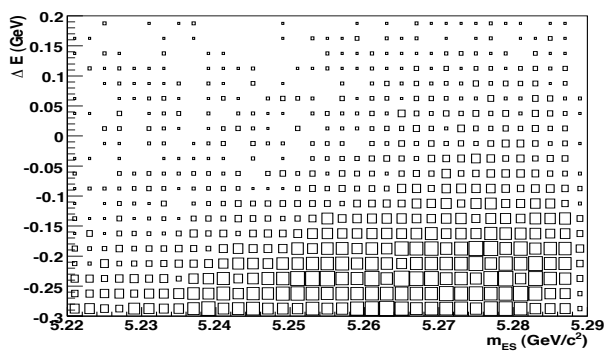

(f) $B \rightarrow K^{*} \gamma X_{s}$ events where the candidate was incorrectly reconstructed ( $X_{s}$ self cross-feed).

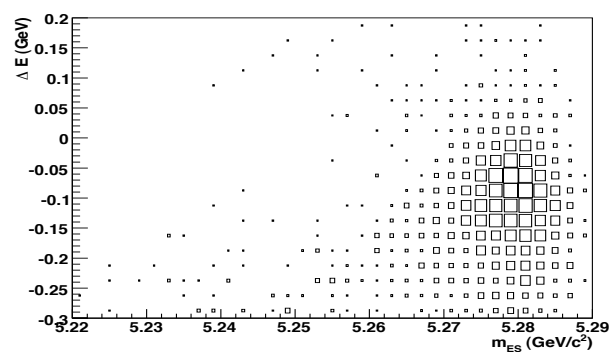

(h) $B \rightarrow X^{s} \gamma$ events where the candidate was correctly reconstructed but the charged kaon was incorrectly identified as a charged pion $\left(X_{s}\right.$ PID crossfeed).

Figure 4.3: $2 \mathrm{D}$ plots of $m_{E S}^{\prime}$ and $\Delta E$ showing $B \rightarrow X_{d}^{L} \gamma$ candidates in each event hypothesis for MC data passing event selection cuts. The number of events in each plots reflects the MC data statistics given in table 3.21. Individual components in the generic $B \bar{B}$ and continuum data are weighted such that the combined data plots reflect any difference in relative contributions expected from those components in experimental 


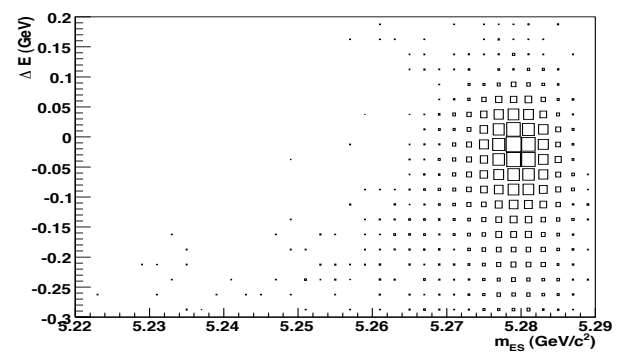

(a) $B \rightarrow X_{d} \gamma \mathrm{KN}$ model events where the candidate was correctly reconstructed.

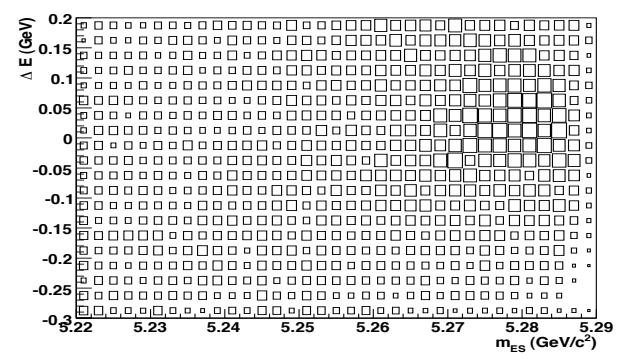

(c) $B \rightarrow \rho / \omega \gamma$ events where the candidate was incorrectly reconstructed (mass cross-feed).

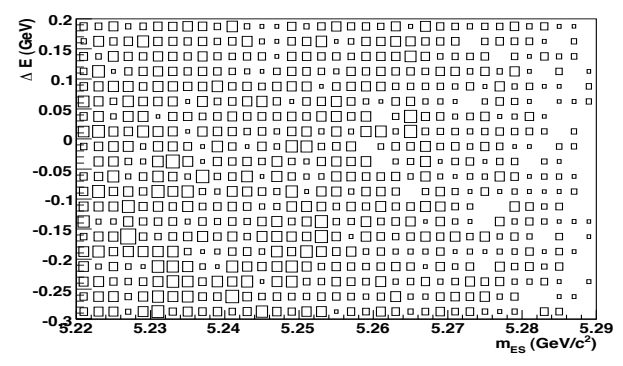

(e) Continuum events.

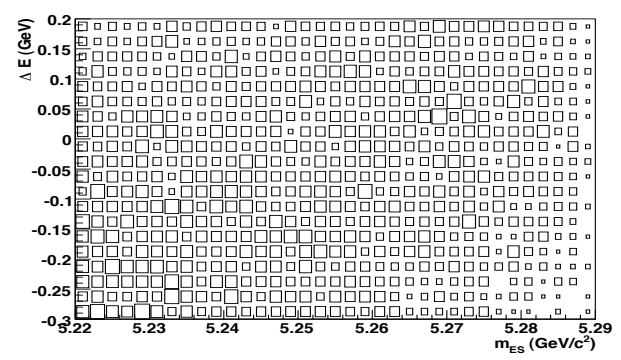

(g) $B \rightarrow K^{*} \gamma$ events where the candidate was incorrectly reconstructed ( $X_{s}$ mass cross-feed).

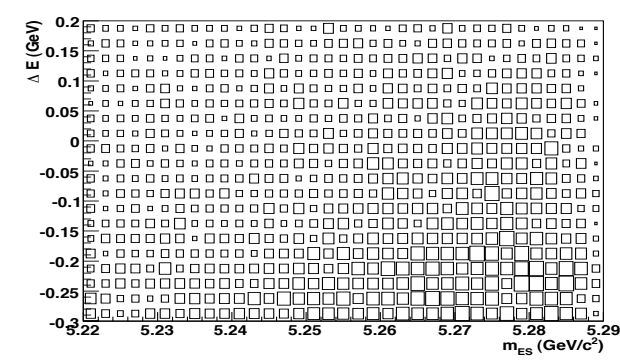

(b) $B \rightarrow X_{d} \gamma \mathrm{KN}$ model events where the candidate was incorrectly reconstructed (self cross-feed).

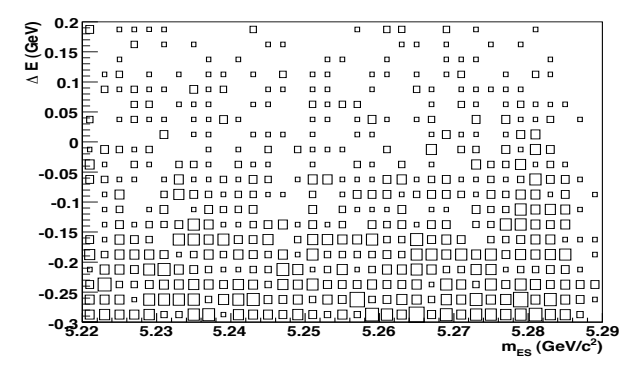

(d) Generic $B \bar{B}$ events.

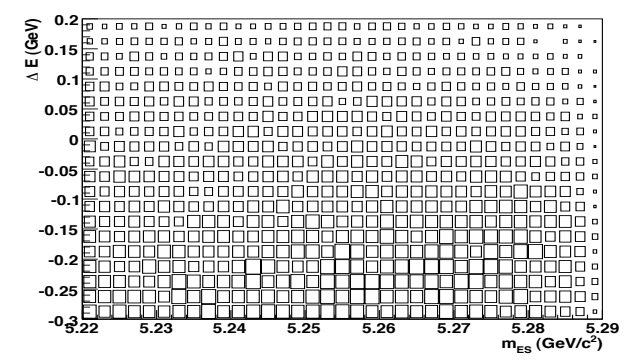

(f) $B \rightarrow X_{s} \gamma \mathrm{KN}$ model events where the candidate was incorrectly reconstructed ( $X_{s}$ self cross-feed).

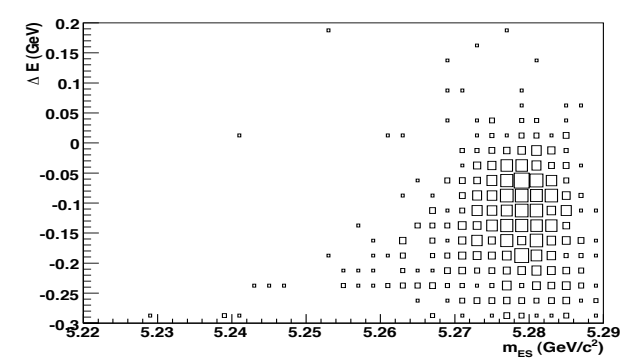

(h) $B \rightarrow X^{s} \gamma$ events where the candidate was correctly reconstructed but the charged kaon was incorrectly identified as a charged pion $\left(X_{s}\right.$ PID crossfeed).

Figure 4.4: 2D plots of $m_{E S}^{\prime}$ and $\Delta E$ showing $B \rightarrow X_{d}^{H} \gamma$ candidates in each event hypothesis for MC data passing event selection cuts. The number of events in each plots reflects the MC data statistics given in table 3.21. Individual components in the generic $B \bar{B}$ and continuum data are weighted such that the combined data plots reflect any difference in relative contributions expected from those components in experimental 
The Argus function [65] is commonly used in high energy physics to model combinatoric backgrounds where the variable phase space has a kinematic threshold. It has the functional form,

$$
\mathcal{P}\left(x ; X_{0}, p, \xi\right)=\frac{1}{\mathcal{N}} x[F(x)]^{p} \exp [\xi F(x)], \quad F(x)=1-\left(\frac{x}{X_{0}}\right)^{2},
$$

where $p$ is a power variable, $\xi$ determines the distribution slope and $X_{0}$ represents an upper kinematic limit. In this analysis the Argus function models combinatoric backgrounds in $m_{E S}^{\prime}$. The threshold is thus defined by the kinematic constraint from the beam energy which fixes $X_{0}=5.29 \mathrm{GeV} / c^{2}$.

The Gaussian function models symmetric peaking distributions and has functional form,

$$
\mathcal{P}(x ; \mu, \sigma)=\frac{1}{\mathcal{N}} \exp \left(-0.5\left(\frac{x-\mu}{\sigma}\right)^{2}\right),
$$

where $\mu$ is the mean and $\sigma$ the width of the distribution.

The Crystal-Ball (CB) [66] function modifies the Gaussian distribution by enhancing the low end tail and therefore improves the modelling of distributions where such a tail exists due to energy loss. It has the following form,

$$
\mathcal{P}(x ; \mu, \sigma, \alpha, n)=\frac{1}{\mathcal{N}} \begin{cases}\exp \left(-0.5\left(\frac{x-\mu}{\sigma}\right)^{2}\right), & \text { for } \frac{x-\mu}{\sigma}>-\alpha \\ \left(\frac{n}{|\alpha|}-|\alpha|-\frac{x-\mu}{\sigma}\right)^{-n} \mathcal{A}(\alpha, n), & \text { for } \frac{x-\mu}{\sigma} \leq-\alpha\end{cases}
$$

where $\mathcal{A}(\alpha, n)=(n /|\alpha|)^{n} \exp \left(-|\alpha|^{2} / 2\right)$. The parameter $\alpha$ determines where the tail intersects the Gaussian distribution and the parameter $n$ describes the shape of the tail.

The 'Cruijff' function [67] modifies the Gaussian distribution by allowing independent widths, $\sigma_{L}$ and $\sigma_{R}$, either side of the mean and allows non-Gaussian tails either side of the mean through the parameters $\alpha_{L}$ and $\alpha_{R}$. It has functional form,

$$
\mathcal{P}\left(x ; \mu, \sigma_{R}, \alpha_{R}, \sigma_{L}, \alpha_{L}\right)=\frac{1}{\mathcal{N}} \begin{cases}\exp \left(-\frac{(x-\mu)^{2}}{2 \sigma_{R}^{2}+\alpha_{R}(x-\mu)^{2}}\right), & \text { for } x \geq \mu \\ \exp \left(-\frac{(x-\mu)^{2}}{2 \sigma_{L}^{2}+\alpha_{L}(x-\mu)^{2}}\right), & \text { for } x<\mu\end{cases}
$$

Finally the polynomial function has the form,

$$
\mathcal{P}\left(x ; a_{1}, . ., a_{N}\right)=\frac{1}{\mathcal{N}}\left(1+\sum_{n=1}^{N} a_{n} x^{n}\right) .
$$

The polynomial order, $N$, is chosen before the MLF. 


\begin{tabular}{|l|l|l|l|}
\hline Hypothesis & Parameter & Low Mass Fit & High Mass Fit \\
\hline Signal & $\mu\left(\mathrm{GeV} / c^{2}\right)$ & $5.27949 \pm 0.00001$ & $5.27942 \pm 0.00001$ \\
& $\sigma\left(\mathrm{GeV} / c^{2}\right)$ & $0.002741 \pm 0.000006$ & $0.002755 \pm 0.000015$ \\
& $\alpha$ & $1.613 \pm 0.013$ & $1.647 \pm 0.026$ \\
& $n$ & $3.926 \pm 0.083$ & $3.74 \pm 0.15$ \\
\hline Generic $B \bar{B}$ & $f$ & $0.42 \pm 0.17$ & $0.19 \pm 0.11$ \\
& $\mu\left(\mathrm{GeV} / c^{2}\right)$ & $5.2793 \pm 0.0017$ & $5.2793 \pm 0.0010$ \\
& $\sigma\left(\mathrm{GeV} / c^{2}\right)$ & $0.0043 \pm 0.0017$ & $0.0047 \pm 0.0013$ \\
& $\xi$ & $-117 \pm 82$ & $-69 \pm 43$ \\
& $p$ & $0.67 \pm 0.80$ & $0.67 \pm 0.71$ \\
\hline Continuum & $\xi$ & $-54 \pm 29$ & $-9.3 \pm 9.3$ \\
& $p$ & $1.04 \pm 0.33$ & $0.553 \pm 0.098$ \\
\hline
\end{tabular}

TABLE 4.2: Fitted $m_{E S}^{\prime}$ PDF parameters in MC data for each event hypothesis showing results for both low and high mass region $B \rightarrow X_{s} \gamma$ candidates.

\subsection{2 $B \rightarrow X_{s} \gamma$ Candidate PDFs}

Signal $B \rightarrow X_{s} \gamma$ events are modelled as a CB function in $m_{E S}^{\prime}$ and a Cruijff function in $\triangle E$ as these PDFs allow for the asymmetries which arise in the signal distributions due to energy leakage. Self cross-feed and mass cross-feed events are modelled as 2D histogram PDFs whose shapes are determined from MC data. Generic $B \bar{B}$ events are modelled as the sum of a Gaussian and Argus function in $m_{E S}^{\prime}$, with the Gaussian having relative normalisation, $f$, and a order-2 polynomial in $\Delta E$. The Gaussian function component in $m_{E S}^{\prime}$ allows for an observed peak in the signal region which can include $B \rightarrow X_{s} \pi^{0}$ decays where the $\pi^{0}$ decayed asymmetrically and the soft photon was not reconstructed resulting in a failure of the $\pi^{0}$ veto used in event selection. Finally continuum events are modelled as an Argus function in $m_{E S}^{\prime}$ and a order-1 polynomial in $\Delta E$.

Figures 4.5 and 4.6 show the fits to $\mathrm{MC}$ data of $m_{E S}^{\prime}$ and $\Delta E$ respectively for $B \rightarrow X_{s} \gamma$ candidates in the low mass region and tables 4.2 and 4.3 list the respective estimator values and associated errors from each PDF fit. The corresponding fits for $B \rightarrow X_{s} \gamma$ candidates in the high mass region are shown in figures 4.7 and 4.8 for $m_{E S}^{\prime}$ and $\Delta E$ respectively with the fitted estimator values and their errors also listed in tables 4.2 and 4.3. Models constructed from 2D histogram PDFs are not fitted to the MC data, instead the MC data histogram fully determines the PDF. It follows that in plots showing these models the PDF and MC data points are entirely coincident. 


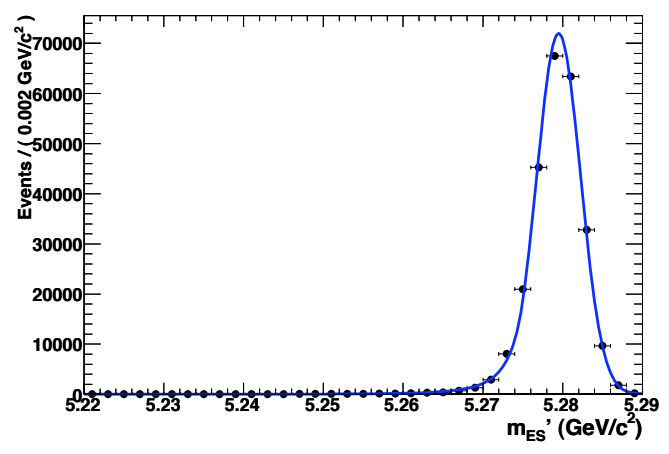

(a) $B \rightarrow K^{*} \gamma$ events with a correctly reconstructed candidate (signal) modelled as a $\mathrm{CB}$ function.

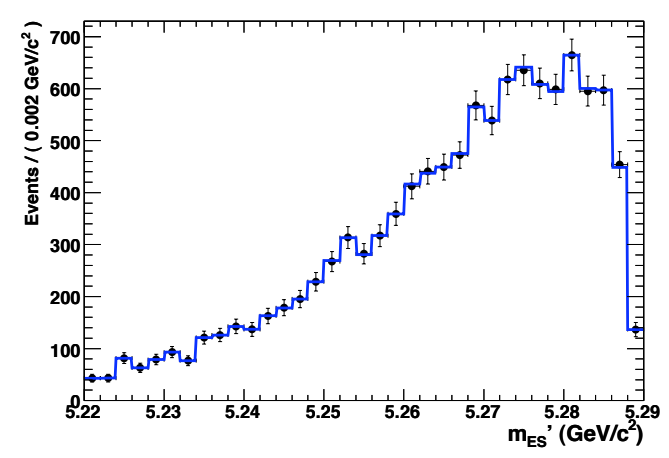

(c) $B \rightarrow X_{s} \gamma \mathrm{KN}$ model events where the candidate was incorrectly reconstructed (mass cross-feed). The $\mathrm{PDF}$ is the $1 \mathrm{D}$ projection of the $2 \mathrm{D}$ histogram.

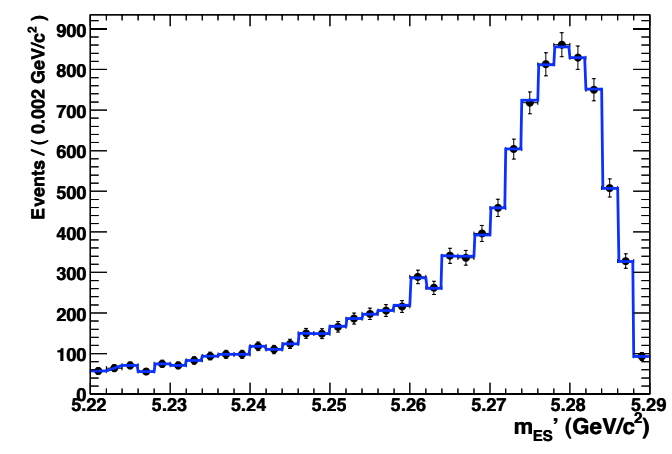

(b) $B \rightarrow K^{*} \gamma$ events where the candidate was incorrectly reconstructed (self cross-feed). The PDF is the $1 \mathrm{D}$ projection of the $2 \mathrm{D}$ histogram.

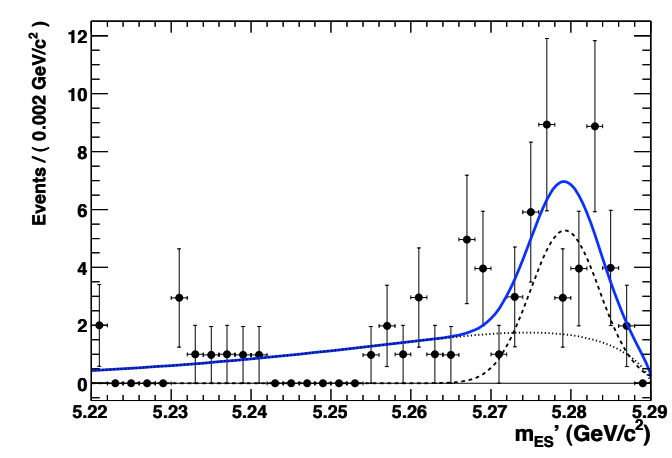

(d) Generic $B \bar{B}$ events modelled as the sum of a Gaussian (dashed) and Argus (dotted) function.

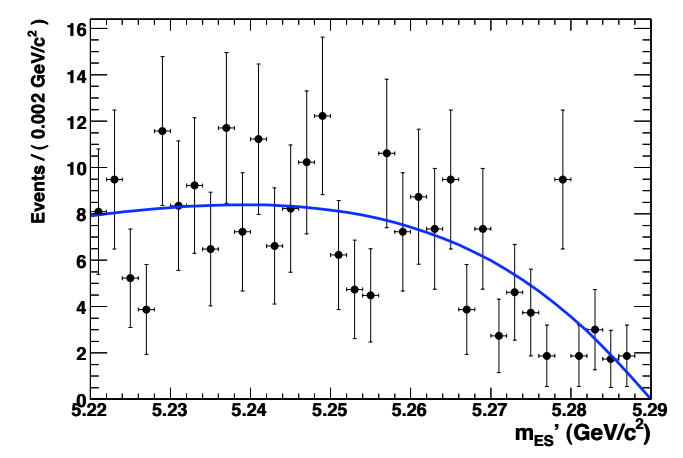

(e) Continuum events modelled as an Argus function.

Figure 4.5: $m_{E S}^{\prime}$ distribution PDF fits to MC data for $B \rightarrow X_{s}^{L} \gamma$ candidates. PDF distributions (solid blue line) are overlaid on the MC data whose error bars reflect the statistical uncertainty from the chosen binning of those data. In all cases the number of events indicated by the ordinate is arbitrarily determined from the MC data statistics. 


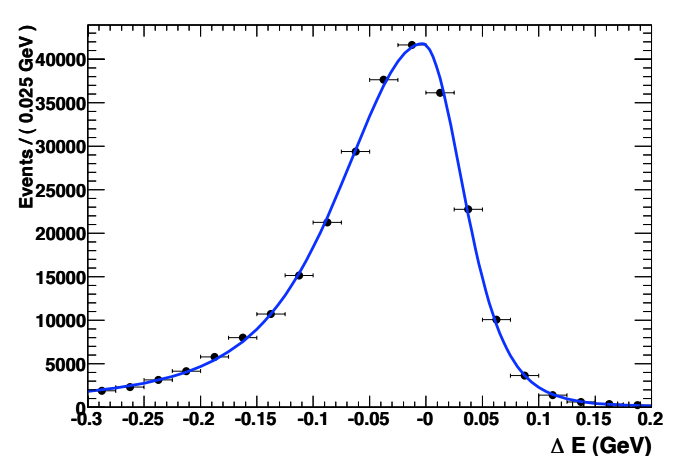

(a) $B \rightarrow K^{*} \gamma$ events with a correctly reconstructed candidate (signal) modelled as a Cruijff function.

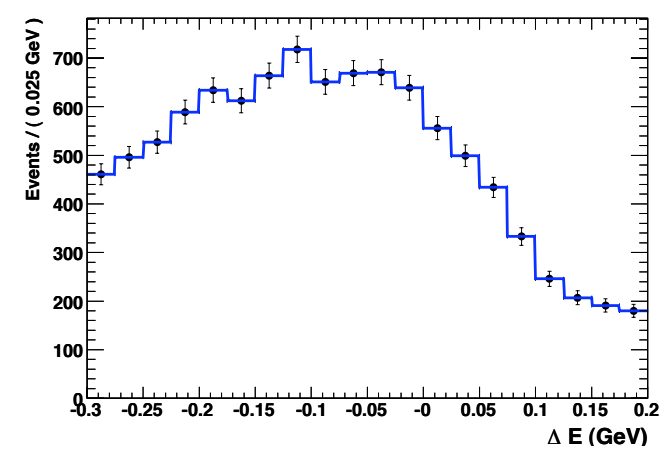

(c) $B \rightarrow X_{s} \gamma \mathrm{KN}$ model events where the candidate was incorrectly reconstructed (mass cross-feed). The $\mathrm{PDF}$ is the $1 \mathrm{D}$ projection of the $2 \mathrm{D}$ histogram.

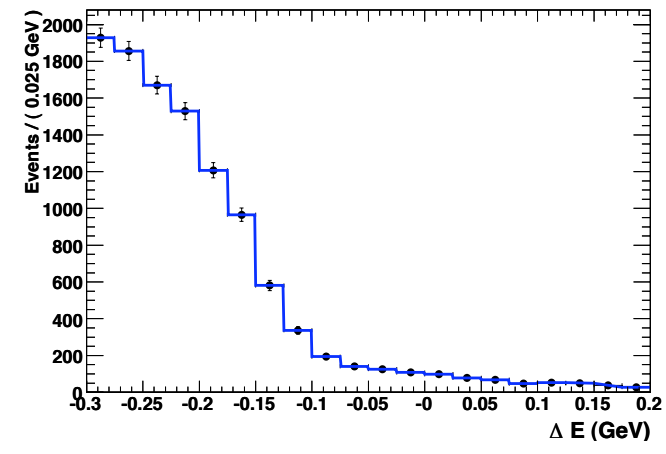

(b) $B \rightarrow K^{*} \gamma$ events where the candidate was incorrectly reconstructed (self cross-feed). The PDF is the $1 \mathrm{D}$ projection of the $2 \mathrm{D}$ histogram.

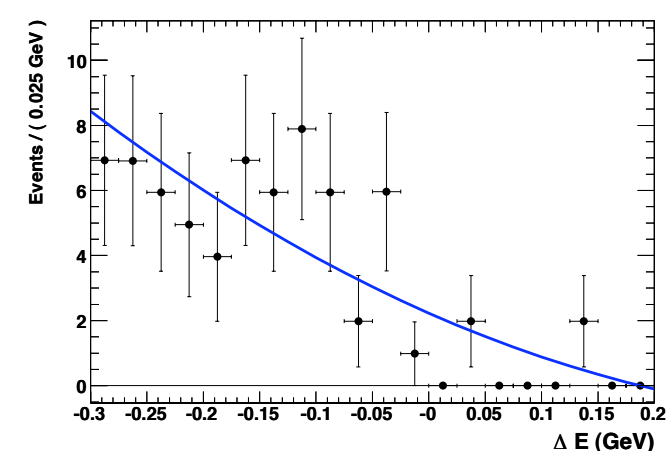

(d) Generic $B \bar{B}$ events modelled as an order-2 polynomial.

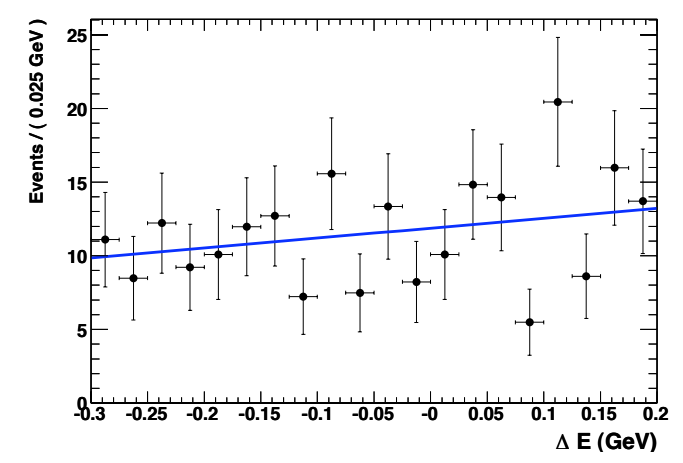

(e) Continuum events modelled as an order-1 polynomial.

Figure 4.6: $\Delta E$ distribution PDF fits to MC data for $B \rightarrow X_{s}^{L} \gamma$ candidates. PDF distributions (solid blue line) are overlaid on the MC data whose error bars reflect the statistical uncertainty from the chosen binning of those data. In all cases the number of events indicated by the ordinate is arbitrarily determined from the MC data statistics. 


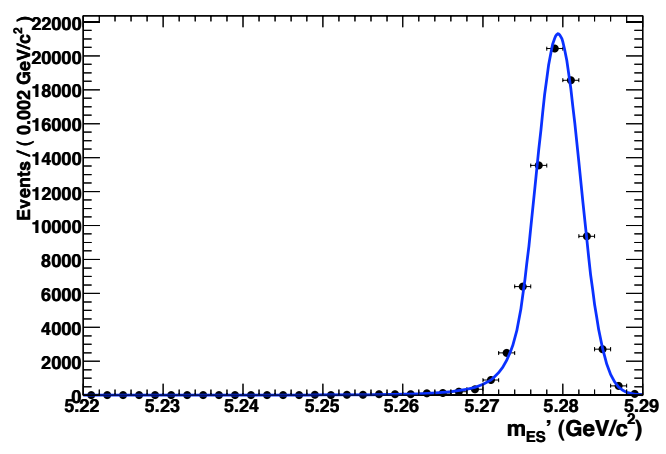

(a) $B \rightarrow X_{s} \gamma \mathrm{KN}$ model events with a correctly reconstructed candidate (signal) modelled as a $\mathrm{CB}$ function.

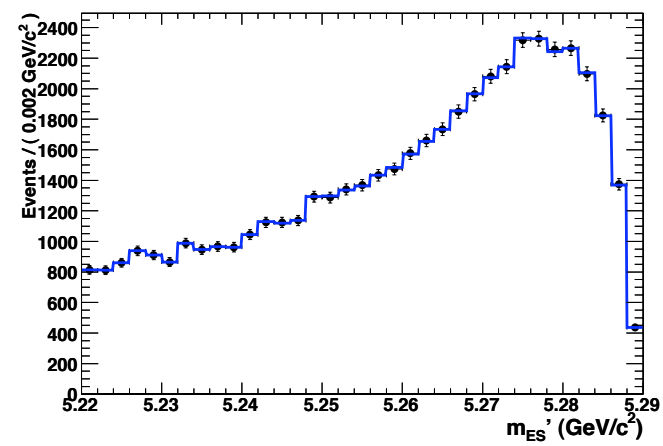

(c) $B \rightarrow K^{*} \gamma$ events where the candidate was incorrectly reconstructed (mass cross-feed). The PDF is the $1 \mathrm{D}$ projection of the $2 \mathrm{D}$ histogram.

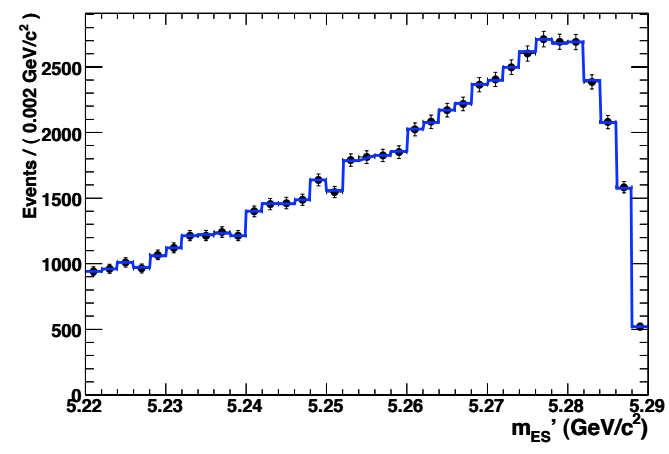

(b) $B \rightarrow X_{s} \gamma \mathrm{KN}$ model events where the candidate was incorrectly reconstructed (self cross-feed). The $\mathrm{PDF}$ is the 1D projection of the $2 \mathrm{D}$ histogram.

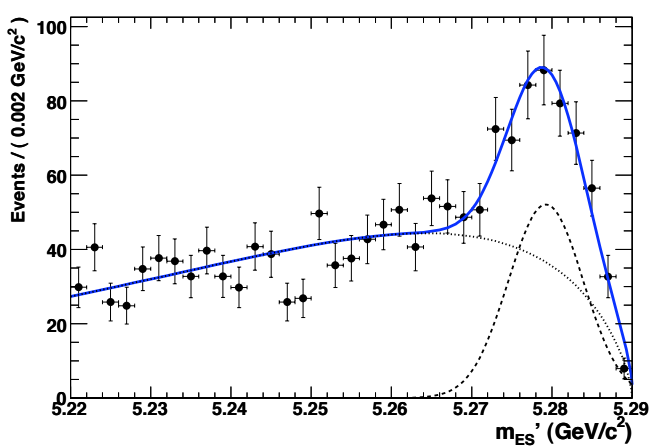

(d) Generic $B \bar{B}$ events modelled as the sum of a Gaussian (dashed) and Argus (dotted) function

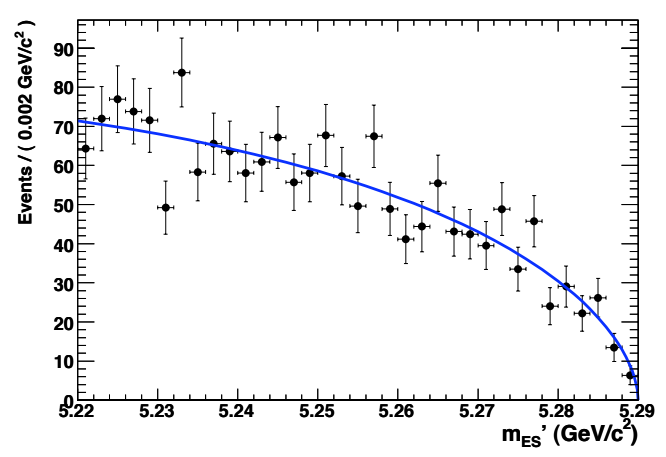

(e) Continuum events modelled as an Argus function.

Figure 4.7: $m_{E S}^{\prime}$ distribution PDF fits to MC data for $B \rightarrow X_{s}^{H} \gamma$ candidates. PDF distributions (solid blue line) are overlaid on the MC data whose error bars reflect the statistical uncertainty from the chosen binning of those data. In all cases the number of events indicated by the ordinate is arbitrarily determined from the MC data statistics. 


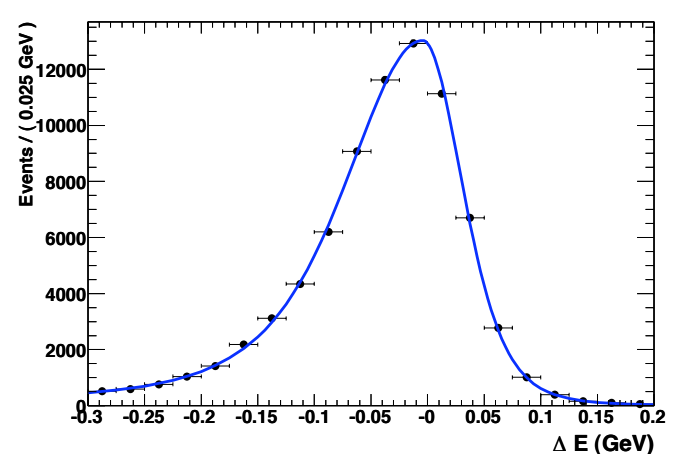

(a) $B \rightarrow X_{s} \gamma \mathrm{KN}$ model events with a correctly reconstructed candidate (signal) modelled as a Cruijff function.

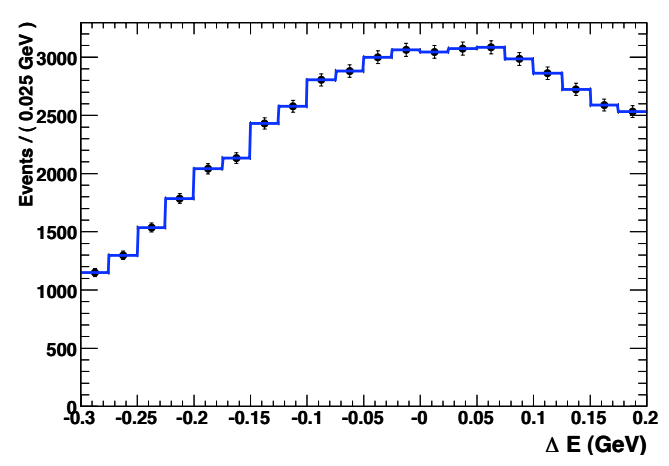

(c) $B \rightarrow K^{*} \gamma$ events where the candidate was incorrectly reconstructed (mass cross-feed). The PDF is the $1 \mathrm{D}$ projection of the $2 \mathrm{D}$ histogram.

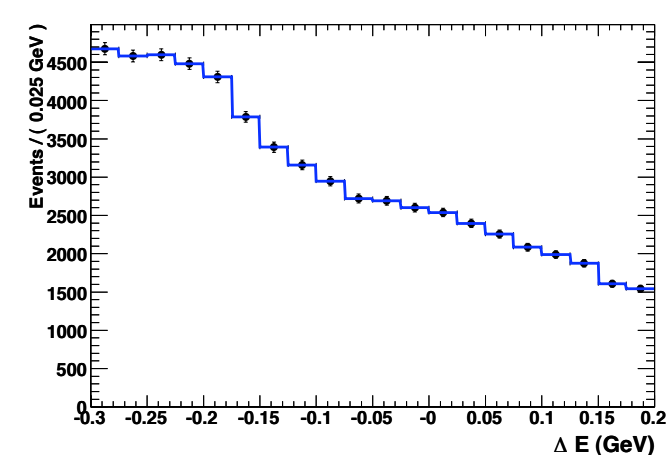

(b) $B \rightarrow X_{s} \gamma \mathrm{KN}$ model events where the candidate was incorrectly reconstructed (self cross-feed). The $\mathrm{PDF}$ is the $1 \mathrm{D}$ projection of the $2 \mathrm{D}$ histogram.

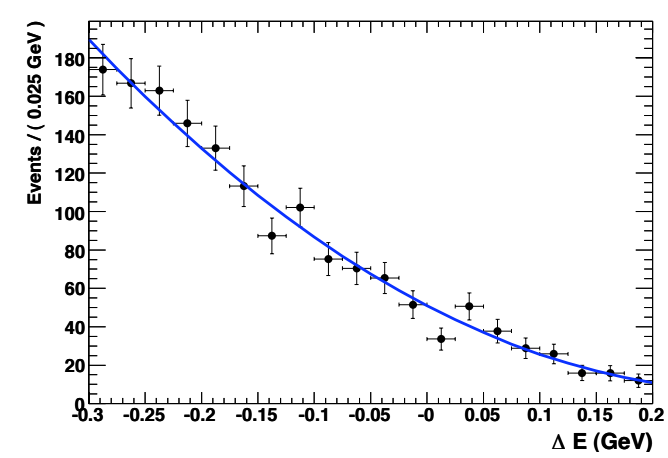

(d) Generic $B \bar{B}$ events modelled as an order-2 polynomial.

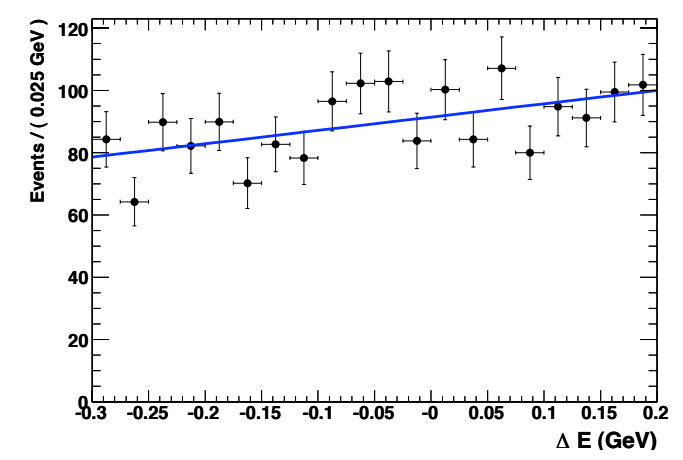

(e) Continuum events modelled as an order-1 polynomial.

Figure 4.8: $\Delta E$ distribution PDF fits to MC data for $B \rightarrow X_{s}^{H} \gamma$ candidates. PDF distributions (solid blue line) are overlaid on the MC data whose error bars reflect the statistical uncertainty from the chosen binning of those data. In all cases the number of events indicated by the ordinate is arbitrarily determined from the MC data statistics. 


\begin{tabular}{|l|l|l|l|}
\hline Hypothesis & Parameter & Low Mass Fit & High Mass Fit \\
\hline Signal & $\mu(\mathrm{GeV})$ & $-0.00307 \pm 0.00037$ & $-0.00445 \pm 0.00068$ \\
& $\sigma_{L}(\mathrm{GeV})$ & $0.06875 \pm 0.00044$ & $0.06483 \pm 0.00075$ \\
& $\sigma_{R}(\mathrm{GeV})$ & $0.03461 \pm 0.00028$ & $0.03412 \pm 0.00052$ \\
& $\alpha_{L}$ & $0.2125 \pm 0.0023$ & $0.2021 \pm 0.0037$ \\
& $\alpha_{L}$ & $0.1189 \pm 0.0020$ & $0.1148 \pm 0.0036$ \\
\hline Generic $B \bar{B}$ & $a_{1}\left(\mathrm{GeV}^{-1}\right)$ & $-6.9 \pm 1.7$ & $-5.99 \pm 0.29$ \\
& $a_{2}\left(\mathrm{GeV}^{-1}\right)$ & $8 \pm 13$ & $10.3 \pm 2.1$ \\
\hline Continuum & $a_{1}\left(\mathrm{GeV}^{-1}\right)$ & $0.57 \pm 0.42$ & $0.47 \pm 0.16$ \\
\hline
\end{tabular}

TABLE 4.3: Fitted $\Delta E$ PDF parameters in MC data for each event hypothesis showing results for both low and high mass region $B \rightarrow X_{s} \gamma$ candidates.

\subsection{3 $\quad B \rightarrow X_{d} \gamma$ Candidate PDFs}

Signal $B \rightarrow X_{d} \gamma$ events are modelled as a CB function in $m_{E S}^{\prime}$ and a Cruijff function in $\Delta E$. Self cross-feed and mass cross-feed events are modelled as 2D histogram PDFs whose shapes are determined from MC data. PID cross-feed events will have very similar distributions to the signal but will be displaced in $\Delta E$ due to the difference in kaon and pion mass. They are therefore also modelled as a CB function in $m_{E S}^{\prime}$ and a Cruijff function in $\triangle E . \quad X_{s}$ self cross-feed and mass cross-feed events are also modelled as $2 \mathrm{D}$ histogram PDFs with shapes determined from MC data. Generic $B \bar{B}$ events are modelled as the sum of a Gaussian and Argus function in $m_{E S}^{\prime}$, with the Gaussian having relative normalisation, $f$, and a order-2 polynomial in $\Delta E$. Finally continuum events are modelled as an Argus function in $m_{E S}^{\prime}$ and a order-1 polynomial in $\Delta E$.

Figures 4.9 and 4.10 show the fits to $\mathrm{MC}$ data of $m_{E S}^{\prime}$ and $\Delta E$ respectively for $B \rightarrow X_{d} \gamma$ candidates in the low mass region and tables 4.4 and 4.5 list the respective estimator values and associated errors calculated from each PDF fit. The corresponding fits for $B \rightarrow X_{d} \gamma$ candidates in the high mass region are shown in figures 4.11 and 4.12 for $m_{E S}^{\prime}$ and $\Delta E$ respectively with the fitted estimator values and their errors also listed in tables 4.4 and 4.5 .

\subsection{Combined Fit Strategy}

To extract the yield for each of the signal types from experimental data four corresponding EMLFs combining the relevant event hypotheses are required. This section describes the implementation of these fits and presents the results of test studies carried out to determine their stability and to check for any potential bias they may introduce. 


\begin{tabular}{|l|l|l|l|}
\hline Hypothesis & Parameter & Low Mass Fit & High Mass Fit \\
\hline Signal & $\mu\left(\mathrm{GeV} / c^{2}\right)$ & $5.27949 \pm 0.00002$ & $5.27939 \pm 0.00004$ \\
& $\sigma\left(\mathrm{GeV} / c^{2}\right)$ & $0.002849 \pm 0.000014$ & $0.002908 \pm 0.000034$ \\
& $\alpha$ & $1.417 \pm 0.023$ & $1.569 \pm 0.067$ \\
& $n$ & $3.85 \pm 0.15$ & $3.21 \pm 0.31$ \\
\hline$X_{s}$ PID cross-feed & $\mu\left(\mathrm{GeV} / c^{2}\right)$ & $5.27918 \pm 0.00016$ & $5.27931 \pm 0.00027$ \\
& $\sigma\left(\mathrm{GeV} / c^{2}\right)$ & $0.00377 \pm 0.00014$ & $0.00378 \pm 0.00021$ \\
& $\alpha$ & $1.32 \pm 0.14$ & $0.82 \pm 0.11$ \\
& $n$ & $2.67 \pm 0.55$ & $14.1 \pm 9.3$ \\
\hline Generic $B \bar{B}$ & $f$ & $0.237 \pm 0.090$ & $0.106 \pm 0.018$ \\
& $\mu\left(\mathrm{GeV} / c^{2}\right)$ & $5.2786 \pm 0.0011$ & $5.28074 \pm 0.00049$ \\
& $\sigma\left(\mathrm{GeV} / c^{2}\right)$ & $0.0034 \pm 0.0012$ & $0.00290 \pm 0.00047$ \\
& $\xi$ & $-69 \pm 33$ & $-49.7 \pm 5.7$ \\
& $p$ & $0.45 \pm 0.32$ & $0.668 \pm 0.053$ \\
\hline Continuum & $\xi$ & $-23 \pm 18$ & $-19.9 \pm 2.9$ \\
& $p$ & $0.67 \pm 0.19$ & $0.6673 \pm 0.0016$ \\
\hline
\end{tabular}

TABLE 4.4: Fitted $m_{E S}^{\prime}$ PDF parameters in MC data for each event hypothesis showing results for both low and high mass region $B \rightarrow X_{d} \gamma$ candidates.

\begin{tabular}{|l|l|l|l|}
\hline Hypothesis & Parameter & Low Mass Fit & High Mass Fit \\
\hline Signal & $\mu(\mathrm{GeV})$ & $-0.00608 \pm 0.00090$ & $-0.0070 \pm 0.0021$ \\
& $\sigma_{L}(\mathrm{GeV})$ & $0.0726 \pm 0.0011$ & $0.0684 \pm 0.0026$ \\
& $\sigma_{R}(\mathrm{GeV})$ & $0.03673 \pm 0.00069$ & $0.0352 \pm 0.0016$ \\
& $\alpha_{L}$ & $0.2338 \pm 0.0060$ & $0.237 \pm 0.014$ \\
& $\alpha_{L}$ & $0.1061 \pm 0.0048$ & $0.118 \pm 0.011$ \\
\hline$X_{s}$ PID cross-feed & $\mu(\mathrm{GeV})$ & $-0.0836 \pm 0.0086$ & $-0.086 \pm 0.010$ \\
& $\sigma_{L}(\mathrm{GeV})$ & $0.081 \pm 0.013$ & $0.155 \pm 0.026$ \\
& $\sigma_{R}(\mathrm{GeV})$ & $0.0459 \pm 0.0061$ & $0.0412 \pm 0.0068$ \\
& $\alpha_{L}$ & $0.24 \pm 0.11$ & $-0.64 \pm 0.34$ \\
& $\alpha_{L}$ & $0.145 \pm 0.028$ & $0.143 \pm 0.031$ \\
\hline Generic $B \bar{B}$ & $a_{1}\left(\mathrm{GeV}^{-1}\right)$ & $-9.5 \pm 1.6$ & $-6.58 \pm 0.38$ \\
& $a_{2}\left(\mathrm{GeV}^{-1}\right)$ & $31 \pm 12$ & $16.7 \pm 2.9$ \\
\hline Continuum & $a_{1}\left(\mathrm{GeV}^{-1}\right)$ & $0.16 \pm 0.28$ & $-0.29 \pm 0.14$ \\
\hline
\end{tabular}

TABLE 4.5: Fitted $\Delta E$ PDF parameters in MC data for each event hypothesis showing results for both low and high mass region $B \rightarrow X_{d} \gamma$ candidates. 


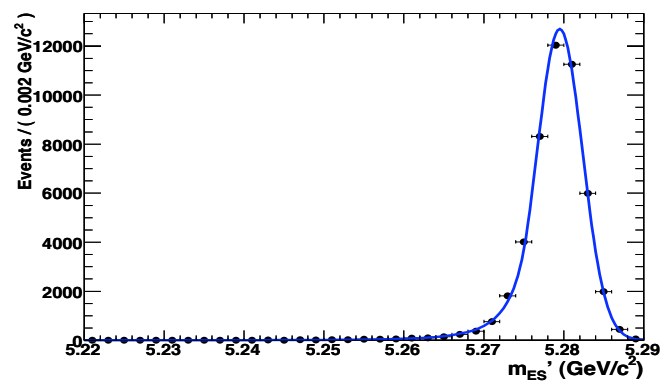

(a) $B \rightarrow \rho / \omega \gamma$ events with a correctly reconstructed candidate (signal) modelled as a CB function.

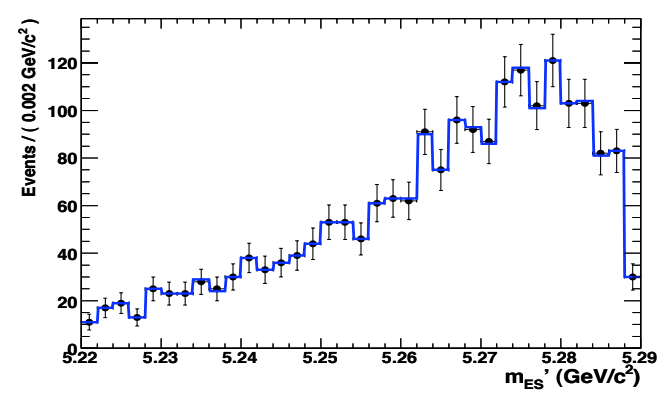

(c) $B \rightarrow X_{d} \gamma \mathrm{KN}$ model events where the candidate was incorrectly reconstructed (mass cross-feed). The $\mathrm{PDF}$ is the $1 \mathrm{D}$ projection of the $2 \mathrm{D}$ histogram.

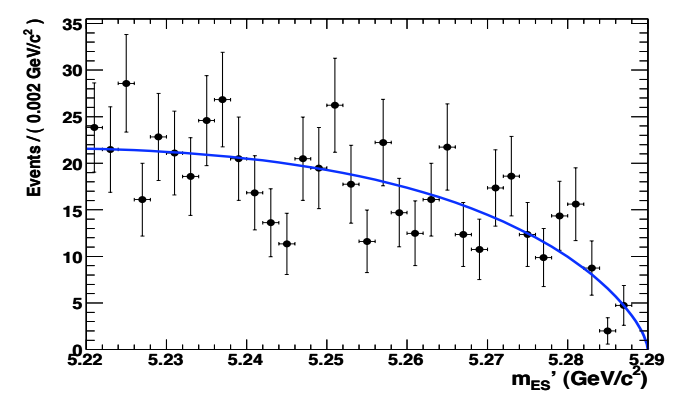

(e) Continuum events modelled as an Argus function.

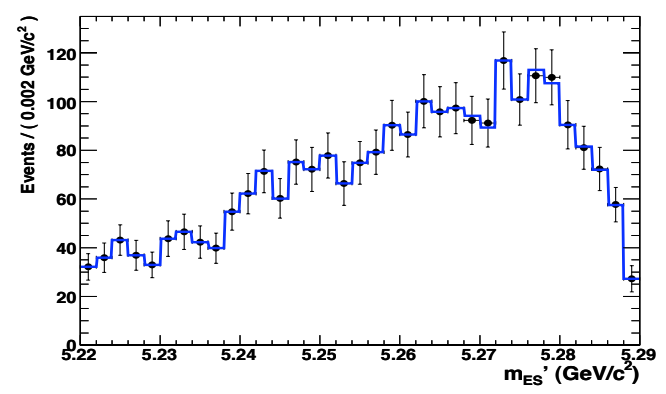

(g) $B \rightarrow X_{s} \gamma$ KN model events where the candidate was incorrectly reconstructed ( $X_{s}$ mass cross-feed). The PDF is the $1 \mathrm{D}$ projection of the $2 \mathrm{D}$ histogram.

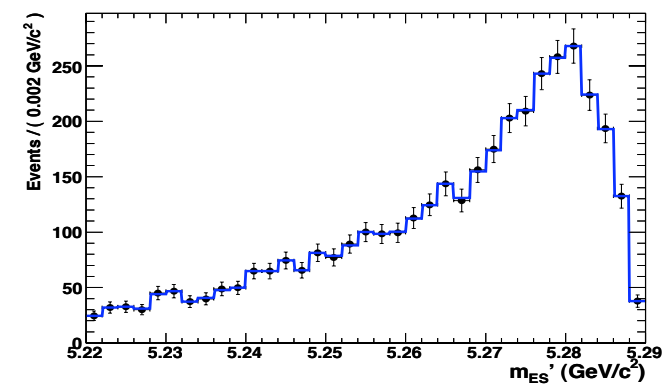

(b) $B \rightarrow \rho / \omega \gamma$ events where the candidate was incorrectly reconstructed (self cross-feed). The PDF is the $1 \mathrm{D}$ projection of the $2 \mathrm{D}$ histogram.

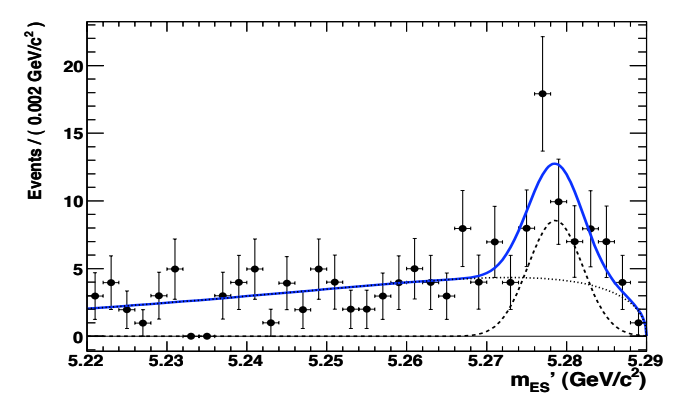

(d) Generic $B \bar{B}$ events modelled as the sum of a Gaussian (dashed) and Argus (dotted) function.

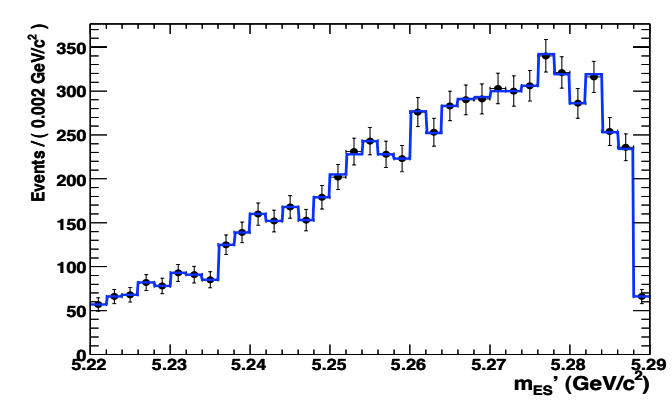

(f) $B \rightarrow K^{*} \gamma$ events where the candidate was incorrectly reconstructed ( $X_{s}$ self cross-feed). The PDF is the $1 \mathrm{D}$ projection of the $2 \mathrm{D}$ histogram.

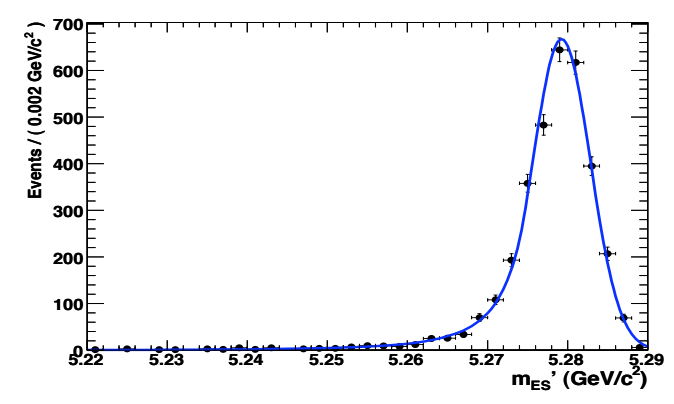

(h) $B \rightarrow X_{s} \gamma$ events with a correctly reconstructed candidate where the charged kaon is incorrectly identified as a charged pion ( $X_{s}$ PID cross-feed). Modelled as a CB function.

Figure 4.9: $m_{E S}^{\prime}$ distribution PDF fits to MC data for $B \rightarrow X_{d}^{L} \gamma$ candidates. PDF distributions (solid blue line) are overlaid on the MC data whose error bars reflect the statistical uncertainty from the chosen binning of those data. In all cases the number of events indicated by the ordinate is arbitrarily determined from the MC data statistics. 


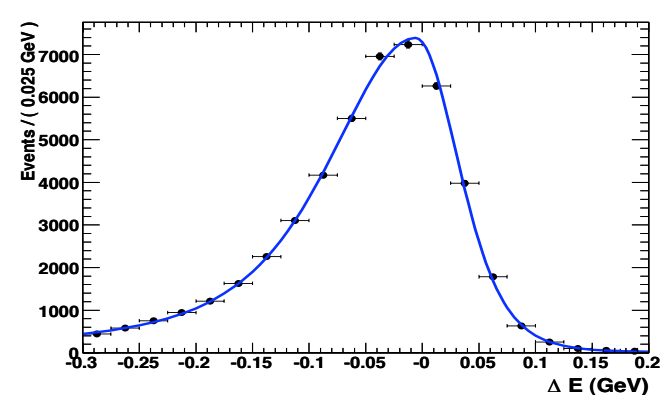

(a) $B \rightarrow \rho / \omega \gamma$ events with a correctly reconstructed candidate (signal) modelled as a Cruijff function.

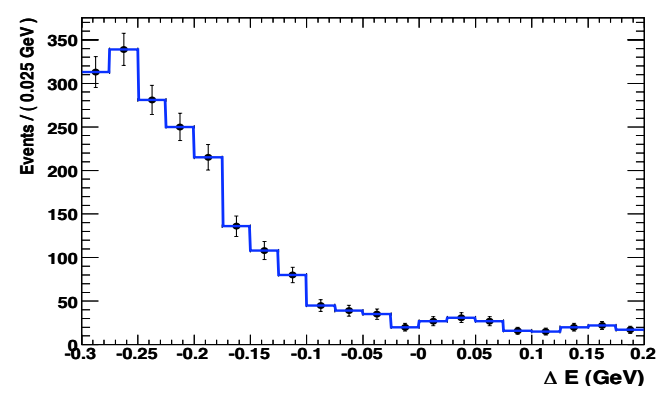

(c) $B \rightarrow X_{d} \gamma \mathrm{KN}$ model events where the candidate was incorrectly reconstructed (mass cross-feed). The $\mathrm{PDF}$ is the $1 \mathrm{D}$ projection of the $2 \mathrm{D}$ histogram.

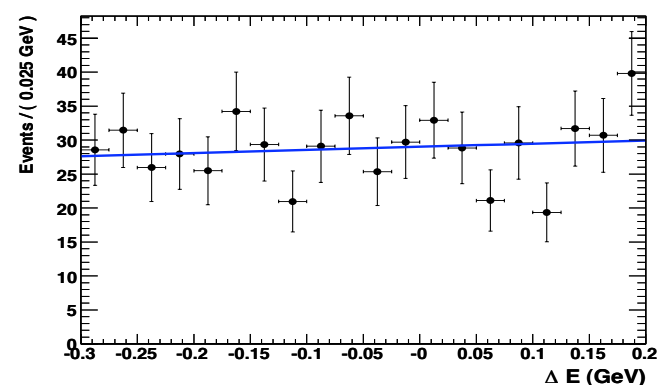

(e) Continuum events modelled as an order-1 polynomial.

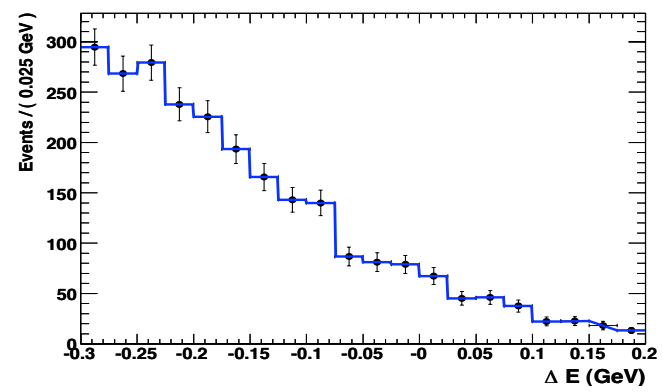

(g) $B \rightarrow X_{s} \gamma$ events where the candidate was incorrectly reconstructed ( $X_{s}$ mass cross-feed). The $\mathrm{PDF}$ is the $1 \mathrm{D}$ projection of the $2 \mathrm{D}$ histogram.

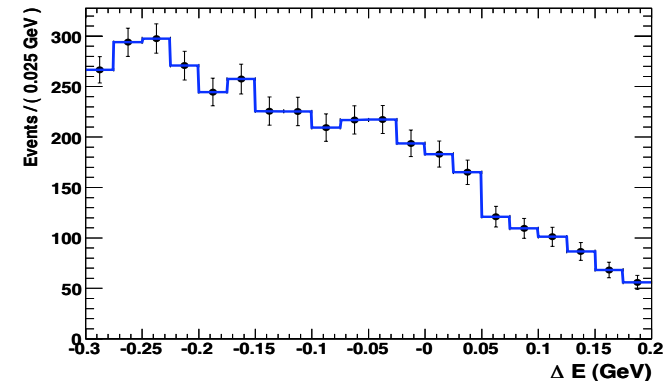

(b) $B \rightarrow \rho / \omega \gamma$ events where the candidate was incorrectly reconstructed (self cross-feed). The PDF is the $1 \mathrm{D}$ projection of the $2 \mathrm{D}$ histogram.

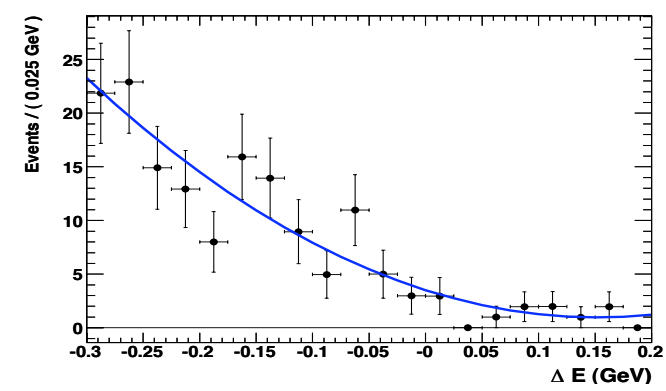

(d) Generic $B \bar{B}$ events modelled as an order-2 polynomial.

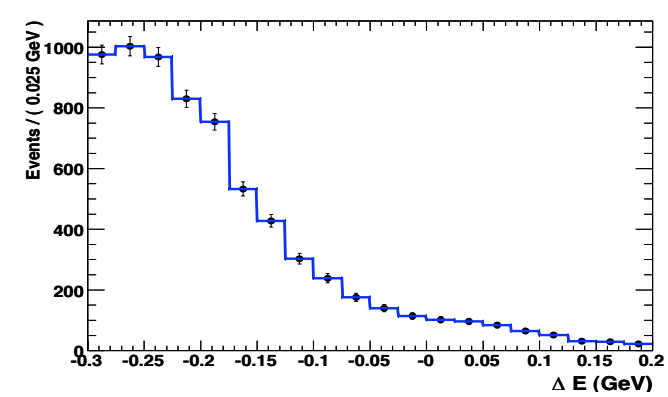

(f) $B \rightarrow K^{*} \gamma$ events where the candidate was incorrectly reconstructed ( $X_{s}$ self cross-feed). The PDF is the $1 \mathrm{D}$ projection of the $2 \mathrm{D}$ histogram.

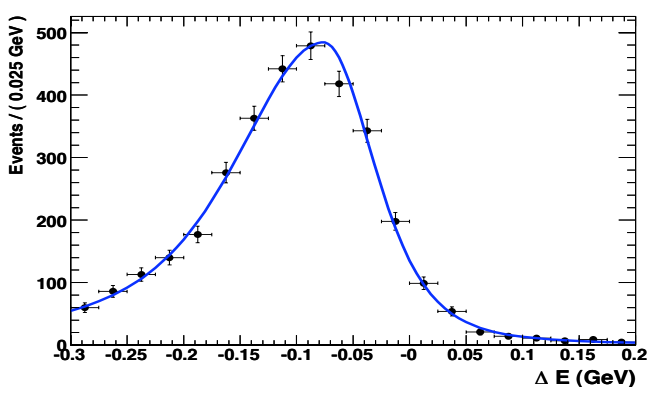

(h) $B \rightarrow X_{s} \gamma$ events with a correctly reconstructed candidate where the charged kaon is incorrectly identified as a charged pion ( $X_{s}$ PID cross-feed). Modelled as a Cruijff function.

Figure 4.10: $\Delta E$ distribution PDF fits to MC data for $B \rightarrow X_{d}^{L} \gamma$ candidates. PDF distributions (solid blue line) are overlaid on the MC data whose error bars reflect the statistical uncertainty from the chosen binning of those data. In all cases the number of events indicated by the ordinate is arbitrarily determined from the MC data statistics. 


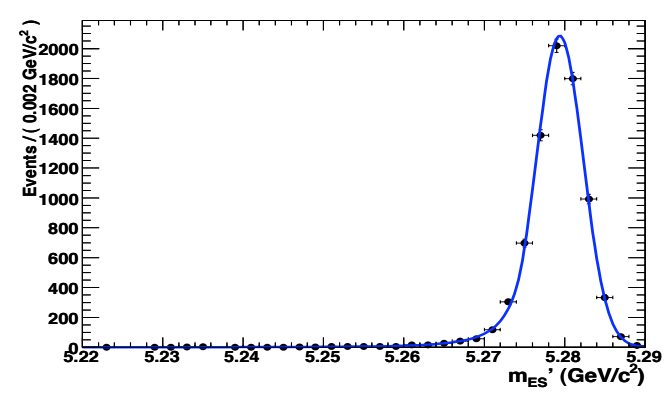

(a) $B \rightarrow X_{d} \gamma \mathrm{KN}$ model events with a correctly reconstructed candidate (signal) modelled as a $\mathrm{CB}$ function.

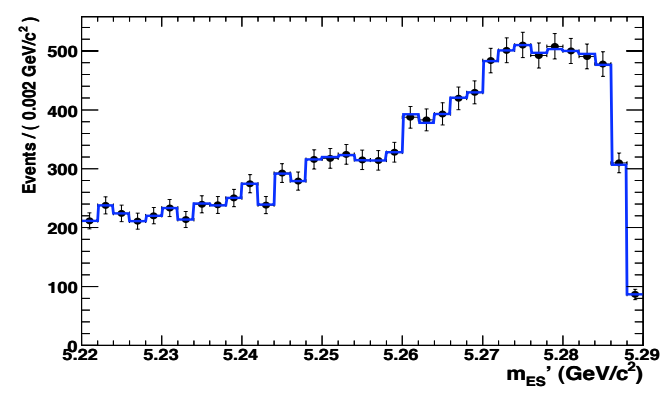

(c) $B \rightarrow \rho / \omega \gamma$ events where the candidate was incorrectly reconstructed (mass cross-feed). The PDF is the $1 \mathrm{D}$ projection of the $2 \mathrm{D}$ histogram.

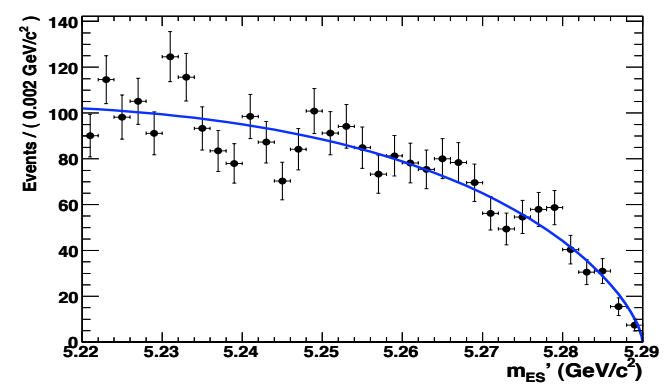

(e) Continuum events modelled as an Argus function.

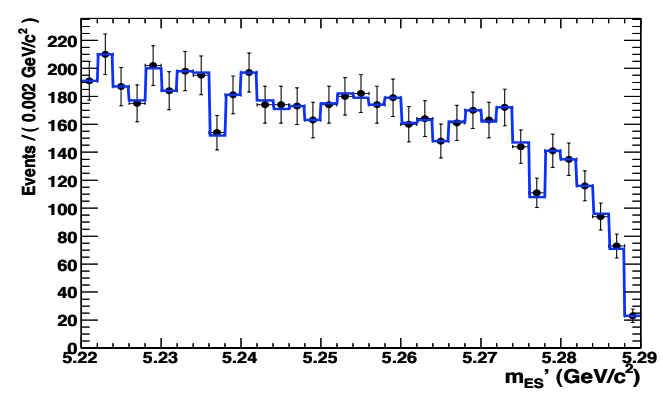

(g) $B \rightarrow K^{*} \gamma$ events where the candidate was incorrectly reconstructed ( $X_{s}$ mass cross-feed). The $\mathrm{PDF}$ is the $1 \mathrm{D}$ projection of the $2 \mathrm{D}$ histogram.

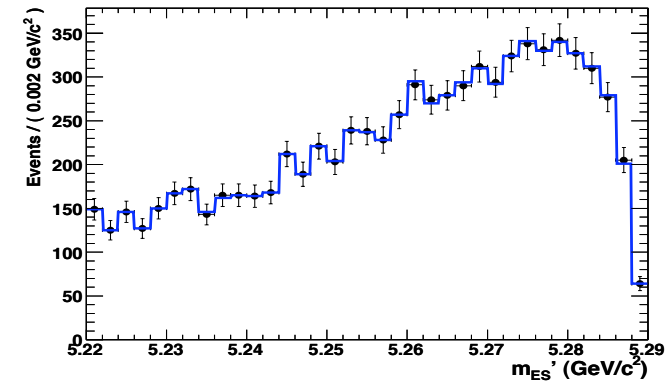

(b) $B \rightarrow X_{d} \gamma \mathrm{KN}$ model events where the candidate was incorrectly reconstructed (self cross-feed). The $\mathrm{PDF}$ is the $1 \mathrm{D}$ projection of the $2 \mathrm{D}$ histogram.

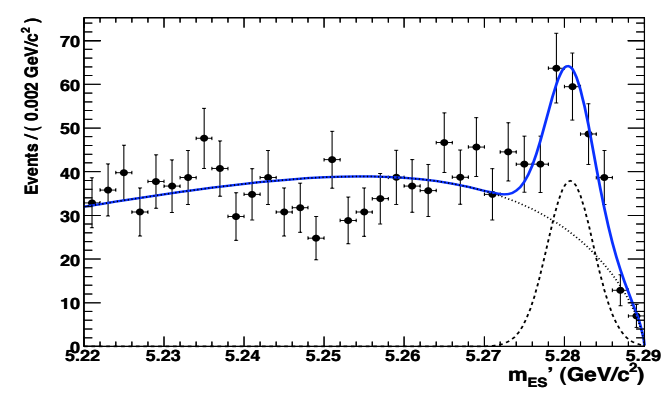

(d) Generic $B \bar{B}$ events modelled as the sum of a Gaussian (dashed) and Argus (dotted) function.

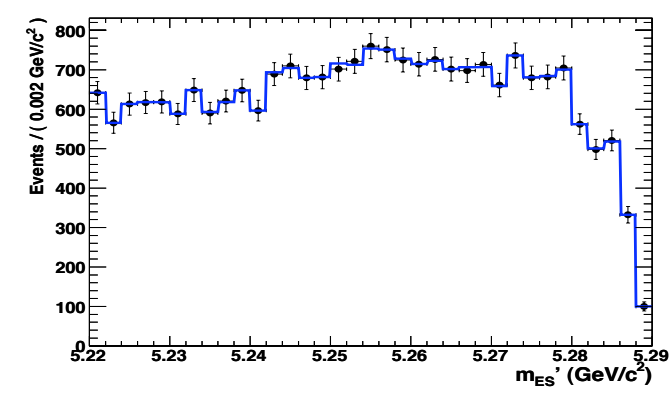

(f) $B \rightarrow X_{s} \gamma \mathrm{KN}$ model events where the candidate was incorrectly reconstructed ( $X_{s}$ self cross-feed). The PDF is the $1 \mathrm{D}$ projection of the $2 \mathrm{D}$ histogram.

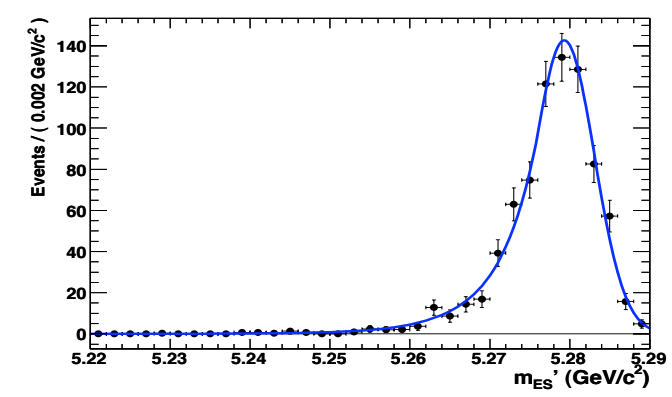

(h) $B \rightarrow X_{s} \gamma$ events with a correctly reconstructed candidate where the charged kaon is incorrectly identified as a charged pion ( $X_{s}$ PID cross-feed). Modelled as a CB function.

Figure 4.11: $m_{E S}^{\prime}$ distribution PDF fits to MC data for $B \rightarrow X_{d}^{H} \gamma$ candidates. PDF distributions (solid blue line) are overlaid on the MC data whose error bars reflect the statistical uncertainty from the chosen binning of those data. In all cases the number of events indicated by the ordinate is arbitrarily determined from the MC data statistics. 


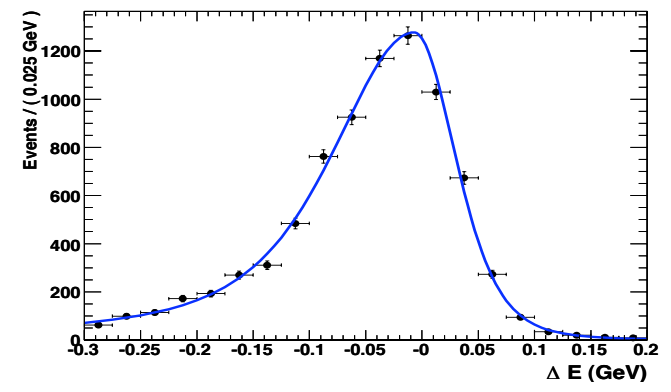

(a) $B \rightarrow X_{d} \gamma \mathrm{KN}$ model events with a correctly reconstructed candidate (signal) modelled as a Cruijff function.

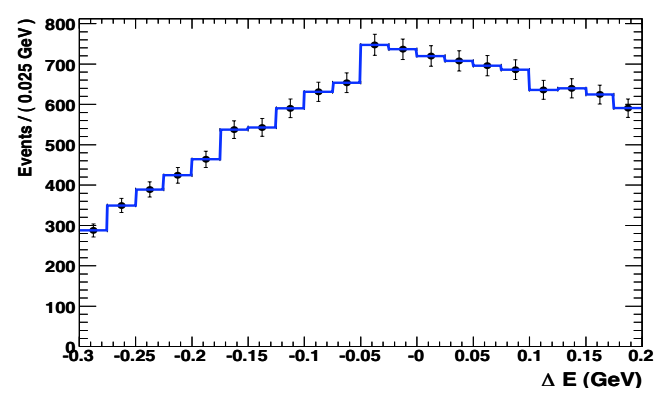

(c) $B \rightarrow \rho / \omega \gamma$ events where the candidate was incorrectly reconstructed (mass cross-feed). The PDF is the $1 \mathrm{D}$ projection of the $2 \mathrm{D}$ histogram.

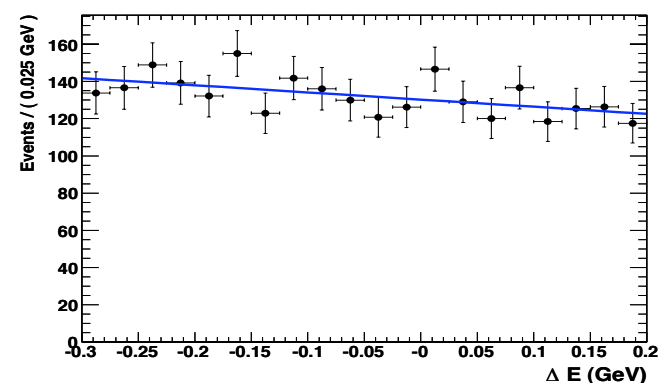

(e) Continuum events modelled as an order-1 polynomial.

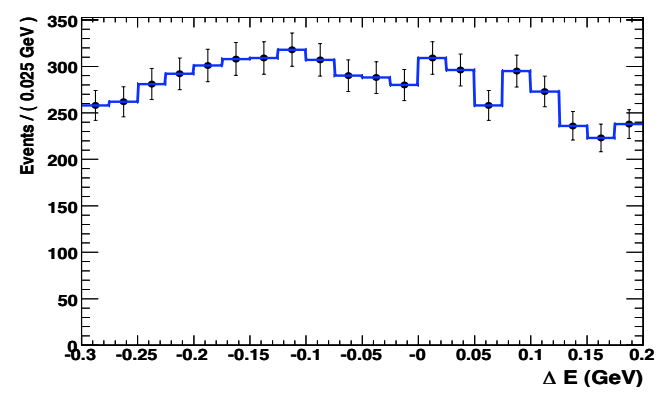

(g) $B \rightarrow K^{*} \gamma$ events where the candidate was incorrectly reconstructed ( $X_{s}$ mass cross-feed). The $\mathrm{PDF}$ is the $1 \mathrm{D}$ projection of the $2 \mathrm{D}$ histogram.

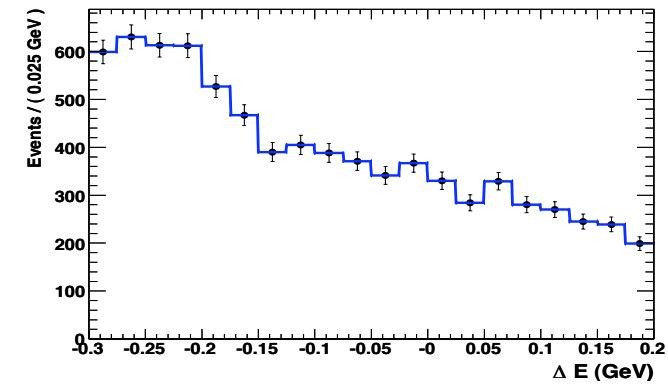

(b) $B \rightarrow X_{d} \gamma \mathrm{KN}$ model events where the candidate was incorrectly reconstructed (self cross-feed). The $\mathrm{PDF}$ is the $1 \mathrm{D}$ projection of the $2 \mathrm{D}$ histogram.

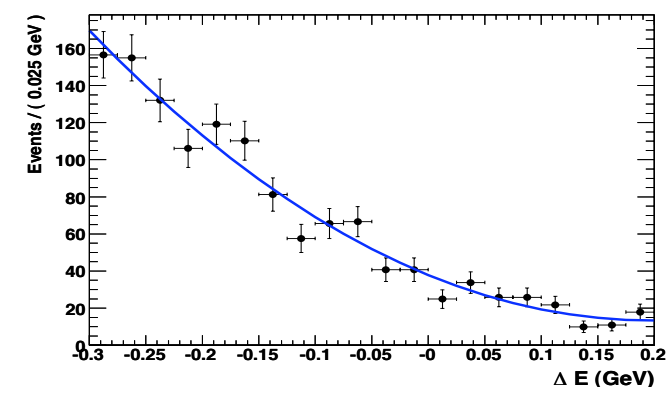

(d) Generic $B \bar{B}$ events modelled as an order-2 polynomial.

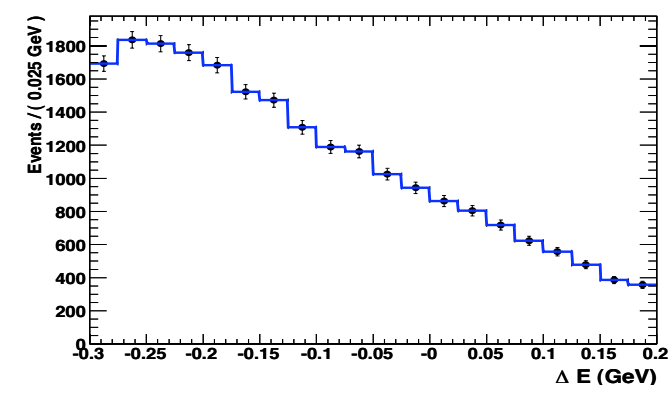

(f) $B \rightarrow X_{s} \gamma \mathrm{KN}$ model events where the candidate was incorrectly reconstructed ( $X_{s}$ self cross-feed). The PDF is the $1 \mathrm{D}$ projection of the $2 \mathrm{D}$ histogram.

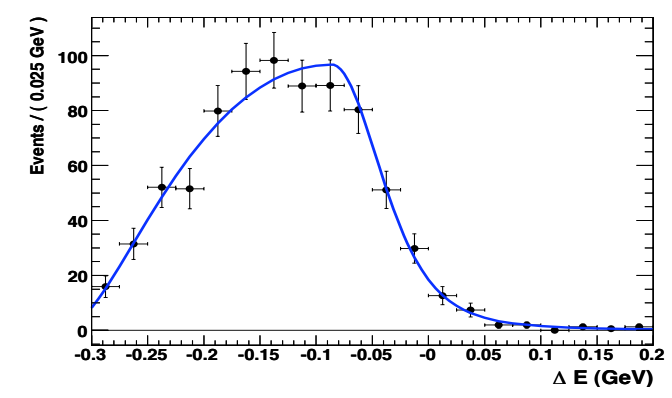

(h) $B \rightarrow X_{s} \gamma$ events with a correctly reconstructed candidate where the charged kaon is incorrectly identified as a charged pion ( $X_{s}$ PID cross-feed). Modelled as a Cruijff function.

Figure 4.12: $\Delta E$ distribution PDF fits to MC data for $B \rightarrow X_{d}^{H} \gamma$ candidates. PDF distributions (solid blue line) are overlaid on the MC data whose error bars reflect the statistical uncertainty from the chosen binning of those data. In all cases the number of events indicated by the ordinate is arbitrarily determined from the MC data statistics. 


\subsubsection{Combined Fit Overview}

The combined fit to experimental data follows the formalism of (4.2) with the EMLF mechanism resulting in the $w_{j}$ weights becoming the mean number of events of each event hypothesis, $n_{j}$. The $n_{j}$ and PDF parameters can either be fixed to pre-determined values or allowed to float in the fit to data. The signal yield, $n_{\text {sig }}$, must be floated as ultimately this is the measurement to be extracted from the experimental data. The choice of which other parameters to float is influenced by factors such as the data sample size, computational power, any correlation between those parameters and knowledge of inadequate modelling in MC data. Particularly important is the stability of the fit; floating too many variables may result in failure to find a stable minimum in $l$ and therefore the fit will not converge. However, for each parameter which is fixed there is a potential of introducing a systematic bias in the signal yield.

Accumulated experience from many years of $B A B A R$ data analysis means there is generally reasonable agreement between simulated data distributions and their experimental data equivalents. However, for correctly reconstructed signal distributions, despite corrections during detector simulation to improve the modelling of energy leakage in the EMC, significant differences between widths in MC and experimental data for $\Delta E$ can still exist. The $\Delta E$ signal width parameters, $\sigma_{L}$ and $\sigma_{R}$, are therefore floated when fitting for $X_{s}$ candidates in both mass regions as the signal contributions in experimental data will be significant enough to sufficiently measure them. The corresponding values for these parameters in $X_{d}$ candidate signal PDFs are then adjusted using the relative difference in $X_{s}$ candidate $\mathrm{MC}$ and experimental data. All remaining signal $\mathrm{MC}$ parameters in $\Delta E$ and $m_{E S}^{\prime}$ are fixed to the values measured in MC data.

Cross-feed normalisations are fixed in fits to data. Previous analyses in the BABAR radiative penguin working group show that the ratio of signal to cross-feed is well modelled by the simulated data and should be preserved when fitting to experimental data [1,30]. Each decay flavour is therefore fitted iteratively between mass regions with the relevant cross-feed yields corrected at each step to preserve their ratio relative to the new fitted signal yield. Iterations continue until the signal yields in both mass regions for that flavour converge. The $B \rightarrow X_{s} \gamma$ candidate data are fitted first and then the relevant fixed cross-feed yields of $B \rightarrow X_{s} \gamma$ events in $B \rightarrow X_{d} \gamma$ are modified to proportionally reflect the signal yields measured in the $B \rightarrow X_{s} \gamma$ data after convergence. Subsequently the $B \rightarrow X_{d} \gamma$ candidate data are fitted for, again iterating between the mass regions and updating the relevant $B \rightarrow X_{d} \gamma$ cross-feed yields at each step until convergence. In $B \rightarrow X_{d} \gamma$ fits the PID cross-feed PDF parameters are fixed to the values measured in MC data. 
Generic $B \bar{B}$ events are generated using decay rate measurements from previous $B A B A R$ analyses and tend to show reasonable agreement between $\mathrm{MC}$ and experimental data. Therefore the normalisations and PDF parameters are fixed using MC data estimates in all fits. Continuum data distributions and normalisations are not so well modelled. In this analysis it is found that floating continuum PDF parameters in the low mass region fits result in them becoming unstable, therefore only the continuum yields are floated and the PDF parameters are fixed to their MC data values. In the high mass region the continuum Argus function shape parameter, $\xi$, and polynomial parameter, $a_{1}$ are also floated as this does not affect the fit stability.

Table 4.6 summarises which PDF parameters and yields are floated and which are fixed in fits to experimental data. The systematic uncertainties which arise from fixing fit components are estimated in chapter 5 . Input values for variables floated in the combined fit to experimental data are determined from the MC data. PDF parameterisation variables are initially set to the values measured in MC data. The signal, cross-feed and continuum yields are initially set to the estimated data yields given in tables $3.22,3.23$ and where applicable table 4.1 .

\subsubsection{Studies to Test for Fit Stability and Bias}

The fits to experimental data are tested for potential bias and stability with 'toy' MC studies. These repeat the fit numerous times with MC data where contributions from each event hypothesis are aggregated into an ensemble dataset intended to reflect the expected event distribution in real data. Such aggregate data can be either 'pure' or 'embedded'. Pure aggregate data is generated randomly, seeding events from the default PDF distributions and normalisations for each event hypothesis in the fit; the generated data therefore reflect those distributions. Embedded aggregate data comprise sub-samples of the fully simulated MC events for a given event hypothesis and normalisation. Fits to pure aggregate data determine the suitability of combining the PDFs used in a given fit, checking for any internal bias. Fits to embedded aggregate data will check the suitability of the PDFs used for those data as correlations in the fully simulated data may not be accounted for in the PDF distributions, hence leading to a bias in the embedded fits. When embedding events the sample size is Poisson distributed about the predetermined normalisation to reflect that an EMLF is to be used.

Toy fits to aggregate data determine the stability of the fit setup; if a significant number of these fits fail to converge then the fit setup will not be suitable for using on experimental data and the strategy must be reconsidered. Furthermore any bias which arises from floating a given parameter can be determined; the floated parameter will 


\begin{tabular}{|c|c|c|c|}
\hline Hypothesis & Category & Fixed & Floated \\
\hline Signal & $\begin{array}{l}\text { Yield } \\
m_{E S} \operatorname{PDF} \\
\Delta E \operatorname{PDF}\left(B \rightarrow X_{s} \gamma\right) \\
\Delta E \operatorname{PDF}\left(B \rightarrow X_{d} \gamma\right)\end{array}$ & $\begin{array}{l}\mu, \sigma, \alpha, n \\
\mu, \alpha_{L}, \alpha_{R} \\
\mu, \alpha_{L}, \alpha_{R}, \sigma_{L}, \sigma_{R}\end{array}$ & $\begin{array}{l}n_{s i g} \\
\sigma_{L}, \sigma_{R}\end{array}$ \\
\hline Self cross-feed & Yield & $n_{s c f}$ (corrected iteratively) & \\
\hline Mass cross-feed & Yield & $n_{m c f}$ (corrected iteratively) & \\
\hline $\begin{array}{l}X_{s} \text { PID cross-feed } \\
\left(B \rightarrow X_{d} \gamma \text { only }\right)\end{array}$ & $\begin{array}{l}\text { Yield } \\
m_{E S} \mathrm{PDF} \\
\Delta E \mathrm{PDF}\end{array}$ & $\begin{array}{l}n_{P I D}^{X s} \\
\mu, \sigma, \alpha, n \\
\mu, \alpha_{L}, \alpha_{R}, \sigma_{L}, \sigma_{R}\end{array}$ & \\
\hline $\begin{array}{l}X_{s} \text { self cross-feed } \\
\left(B \rightarrow X_{d} \gamma \text { only }\right)\end{array}$ & Yield & $n_{s c f}^{X s}$ & \\
\hline $\begin{array}{l}X_{s} \text { mass cross-feed } \\
\left(B \rightarrow X_{d} \gamma \text { only }\right)\end{array}$ & Yield & $n_{m c f}^{X s}$ & \\
\hline Generic $B \bar{B}$ & $\begin{array}{l}\text { Yield } \\
m_{E S} \mathrm{PDF} \\
\Delta E \mathrm{PDF} \\
\end{array}$ & $\begin{array}{l}n_{G e n B} \\
\xi, p, f, \mu, \sigma \\
a_{1}, a_{2}\end{array}$ & \\
\hline Continuum & $\begin{array}{l}\text { Yield } \\
m_{E S} \operatorname{PDF}\left(B \rightarrow X^{L} \gamma\right) \\
\Delta E \operatorname{PDF}\left(B \rightarrow X^{L} \gamma\right) \\
m_{E S} \operatorname{PDF}\left(B \rightarrow X^{H} \gamma\right) \\
\Delta E \operatorname{PDF}\left(B \rightarrow X^{H} \gamma\right)\end{array}$ & $\begin{array}{l}\xi, p \\
a_{1} \\
p\end{array}$ & $\begin{array}{l}n_{\text {cont }} \\
\xi \\
a_{1} \\
\end{array}$ \\
\hline
\end{tabular}

TABLE 4.6: Summary of fixed and floated event class yields and PDF parameters in fits to experimental data.

have a known value when the aggregate data is generated, $\alpha_{\text {gen }}$. The value returned from the toy fit, $\alpha_{f i t}$, will then have an associated 'pull' which measures the difference in the generated and fitted values scaled by the error on that parameter calculated from the fit, $\sigma_{\alpha}$; explicitly the pull is defined as $\left(\alpha_{g e n}-\alpha_{f i t}\right) / \sigma_{\alpha}$. For an unbiased fit the pull distribution accumulated from numerous identical fits to different aggregate data samples will approach a Gaussian distribution centred on zero with unit width. Any departure from this distribution indicates internal bias in the fit which systematically biases the parameter under consideration. Such biases can result from an ill-defined fit strategy (for example fixing one parameter which is highly correlated with a floated parameter) or simply due to too few statistics for a given event hypothesis. This bias must be removed by revising the fit strategy or corrected for after the fit to data. In this analysis only potential bias on the signal yield is corrected for.

For each of the four fits to be carried out on experimental data, 500 toy fits to pure aggregate data are performed to check the suitability of combining the different event 
hypothesis PDFs in the fit. Additionally 500 toy fits embedding signal and cross-feed simulated data are performed to investigate potential bias in the floated yields due to these data. The resulting pull distribution histograms are fitted with a Gaussian function using a $\chi^{2}$ minimisation technique [63].

The toy study framework does not allow the iteration between mass regions to correct cross-feed components to be studied, so the cross-feed yields are additionally varied as part of the systematic studies described in chapter 5. Furthermore the framework does not persist the weighted corrections made to $B \rightarrow X_{s} \gamma$ phase space decays described in section 4.2 so all embedded studies are carried out with PDF distributions and normalisations determined from unweighted MC data. It is not believed that weighting the MC data will have a significant effect on the signal yield bias, so the unweighted embedded studies are still considered a robust investigation of fit bias. The pure toy studies do use the weighted MC data distributions and normalisations.

\subsubsection{1 $\quad X_{s}$ Candidate Fits}

Figure 4.13 shows the signal yield pull histograms and fitted Gaussian distributions from aggregate MC data toy studies for fits to low and high mass $B \rightarrow X_{s} \gamma$ candidates. The embedded toy studies require the signal, self cross-feed and mass cross-feed components to be sampled from fully simulated MC data and the remaining event hypotheses generated as pure aggregate MC data. Tables 4.7 and 4.8 list the fitted Gaussian parameters, $\mu$ and $\sigma$, to pull histograms for all components floated in the respective low and high mass fits. Also shown are the average correlation coefficients, $\rho_{\text {sig }}$, between each floated variable and the signal yield calculated from the embedded toy studies. In all cases the signal yield distributions are deemed acceptable and the average pull on the embedded signal yield distributions will be used to correct the signal yield obtained from fits to experimental data. Pulls calculated from each floated variable in the embedded study are used to estimate systematic uncertainties due to fit bias in section 5.1.3.4. Low correlation coefficients mean that the relatively large pulls observed for some signal PDF parameters are expected to have a relatively small effect on $n_{\text {sig }}$ and so are not a significant concern. All fits performed in these studies converged successfully.

\subsubsection{2 $\quad X_{d}$ Candidates}

Figure 4.14 shows the signal yield pull histograms and fitted Gaussian distributions from aggregate MC data toy studies for the fits to low and high mass $B \rightarrow X_{d} \gamma$ candidates. Two distinct embedded toy studies are carried out. Initially the signal, $X_{d}$ self crossfeed and $X_{d}$ mass cross-feed components are sampled from fully simulated MC data 


\begin{tabular}{|c|c|c|c|c|}
\hline \multicolumn{2}{|c|}{ Parameter } & Pure Study & Embedded Study & $\rho_{\text {sig }}$ \\
\hline \multirow[t]{2}{*}{$n_{\text {sig }}$} & $\mu$ & $0.038 \pm 0.047$ & $-0.127 \pm 0.048$ & 1.000 \\
\hline & $\sigma$ & $0.981 \pm 0.037$ & $0.999 \pm 0.037$ & \\
\hline \multirow[t]{2}{*}{$n_{\text {cont }}$} & $\mu$ & $-0.106 \pm 0.047$ & $-0.092 \pm 0.047$ & -0.069 \\
\hline & $\sigma$ & $0.978 \pm 0.044$ & $0.998 \pm 0.037$ & \\
\hline \multirow[t]{2}{*}{$\sigma_{L}$} & $\mu$ & $-0.003 \pm 0.048$ & $-0.461 \pm 0.054$ & 0.098 \\
\hline & $\sigma$ & $0.983 \pm 0.035$ & $1.100 \pm 0.043$ & \\
\hline \multirow[t]{2}{*}{$\sigma_{R}$} & $\mu$ & $0.070 \pm 0.051$ & $-0.282 \pm 0.045$ & 0.042 \\
\hline & $\sigma$ & $1.012 \pm 0.040$ & $0.964 \pm 0.034$ & \\
\hline
\end{tabular}

TABLE 4.7: Fitted Gaussian parameters to pull distributions from toy MC studies for all floated parameters in the low mass $B \rightarrow X_{s} \gamma$ fit. Also shown are the average correlation coefficients between each floated variable and $n_{\text {sig }}$ for the embedded toy studies.

\begin{tabular}{|c|c|c|c|c|}
\hline \multicolumn{2}{|c|}{ Parameter } & Pure Study & Embedded Study & $\rho_{\text {sig }}$ \\
\hline \multirow[t]{2}{*}{$n_{\text {sig }}$} & $\mu$ & $-0.050 \pm 0.044$ & $0.017 \pm 0.045$ & 1.000 \\
\hline & $\sigma$ & $0.924 \pm 0.039$ & $0.927 \pm 0.036$ & \\
\hline \multirow[t]{2}{*}{$n_{\text {cont }}$} & $\mu$ & $-0.030 \pm 0.044$ & $0.108 \pm 0.045$ & -0.202 \\
\hline & $\sigma$ & $0.947 \pm 0.034$ & $0.930 \pm 0.035$ & \\
\hline \multirow[t]{2}{*}{$\sigma_{L}$} & $\mu$ & $-0.077 \pm 0.049$ & $-0.834 \pm 0.046$ & 0.233 \\
\hline & $\sigma$ & $1.014 \pm 0.039$ & $0.954 \pm 0.034$ & \\
\hline \multirow[t]{2}{*}{$\sigma_{R}$} & $\mu$ & $-0.097 \pm 0.043$ & $-0.326 \pm 0.047$ & 0.167 \\
\hline & $\sigma$ & $0.926 \pm 0.035$ & $0.970 \pm 0.038$ & \\
\hline \multirow[t]{2}{*}{$\xi$} & $\mu$ & $-0.047 \pm 0.047$ & $0.002 \pm 0.043$ & 0.251 \\
\hline & $\sigma$ & $0.968 \pm 0.036$ & $0.895 \pm 0.036$ & \\
\hline \multirow[t]{2}{*}{$a_{1}$} & $\mu$ & $-0.003 \pm 0.046$ & $-0.135 \pm 0.050$ & 0.048 \\
\hline & $\sigma$ & $0.943 \pm 0.037$ & $1.042 \pm 0.041$ & \\
\hline
\end{tabular}

TABLE 4.8: Fitted Gaussian parameters to pull distributions from toy MC studies for all floated parameters in the high mass $B \rightarrow X_{s} \gamma$ fit. Also shown are the average correlation coefficients between each floated variable and $n_{\text {sig }}$ for the embedded toy studies.

and the remaining event hypotheses are generated as pure aggregate MC data. Finally the signal, $X_{d}$ self cross-feed, $X_{d}$ mass cross-feed, $X_{s}$ PID cross-feed, $X_{s}$ self cross-feed and $X_{s}$ mass cross-feed components are sampled from fully simulated MC data and the remaining event hypotheses are generated as pure aggregate MC data. Tables 4.9 and 4.10 list the fitted Gaussian parameters, $\mu$ and $\sigma$, to pull histograms for all components floated in the respective low and high mass regions for these toy studies. Also shown are the average correlation coefficients between each floated variable and $n_{\text {sig }}$ for the embedded toy study where both $X_{d}$ and $X_{s}$ MC data are embedded. In all cases the distributions are deemed acceptable and the average pull on the signal yield distribution with both $X_{d}$ and $X_{s}$ cross-feed events embedded will be used to correct the signal yield 


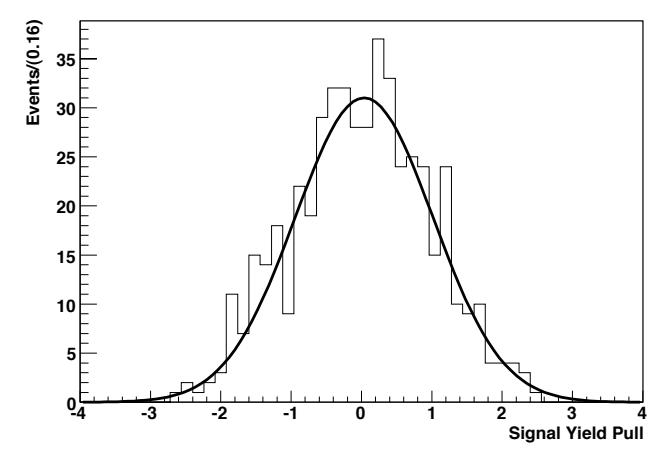

(a) Low mass pure toy signal yield pull.

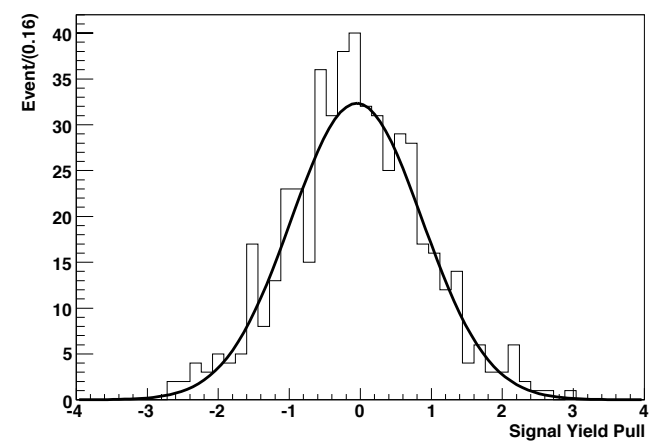

(c) High mass pure toy signal yield pull.

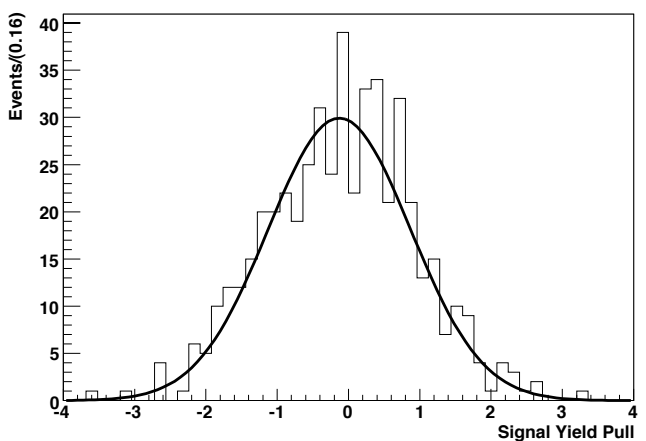

(b) Low mass embedded signal and $X_{s}$ cross-feed toy signal yield pull.

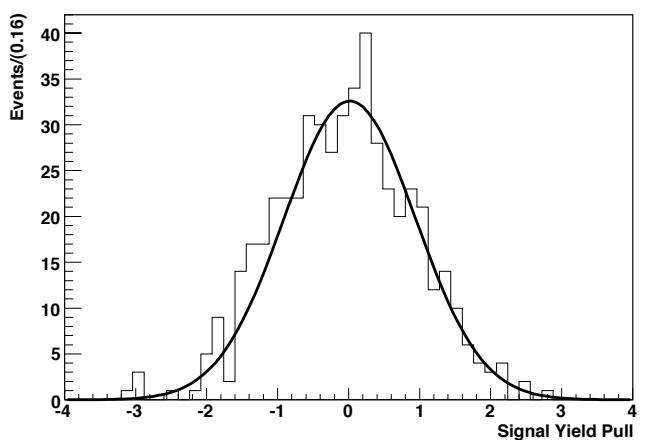

(d) High mass embedded signal and $X_{s}$ cross-feed toy signal yield pull.

Figure 4.13: Toy study signal yield pull histograms and fitted Gaussian distributions for $B \rightarrow X_{s} \gamma$ candidate fits to experimental data. Each study consists of 500 toy fits.

\begin{tabular}{|c|c|c|c|c|c|}
\hline \multicolumn{2}{|c|}{ Parameter } & Pure Study & Embedded Study & Embedded $X_{s}$ Study & $\rho_{\text {sig }}$ \\
\hline \multirow[t]{2}{*}{$n_{\text {sig }}$} & $\mu$ & $-0.182 \pm 0.049$ & $-0.021 \pm 0.048$ & $0.109 \pm 0.045$ & \multirow[t]{2}{*}{1.000} \\
\hline & $\sigma$ & $1.033 \pm 0.038$ & $1.010 \pm 0.035$ & $0.940 \pm 0.041$ & \\
\hline \multirow[t]{2}{*}{$n_{\text {cont }}$} & $\mu$ & $0.051 \pm 0.049$ & $0.009 \pm 0.048$ & $-0.025 \pm 0.045$ & \multirow[t]{2}{*}{-0.151} \\
\hline & $\sigma$ & $1.051 \pm 0.042$ & $1.001 \pm 0.039$ & $0.947 \pm 0.036$ & \\
\hline
\end{tabular}

TABLE 4.9: Fitted Gaussian parameters to pull distributions from toy MC studies for all floated parameters in the low mass $B \rightarrow X_{d} \gamma$ fit. Also shown are the average correlation coefficients between each floated variable and $n_{\text {sig }}$ for the embedded toy study where both $X_{d}$ and $X_{s}$ MC data are embedded.

obtained from fits to experimental data. Pulls from each floated variable in this full embedded study are used to evaluate a fit bias systematic uncertainty in section 5.1.3.4. All fits performed in these studies converged successfully. 


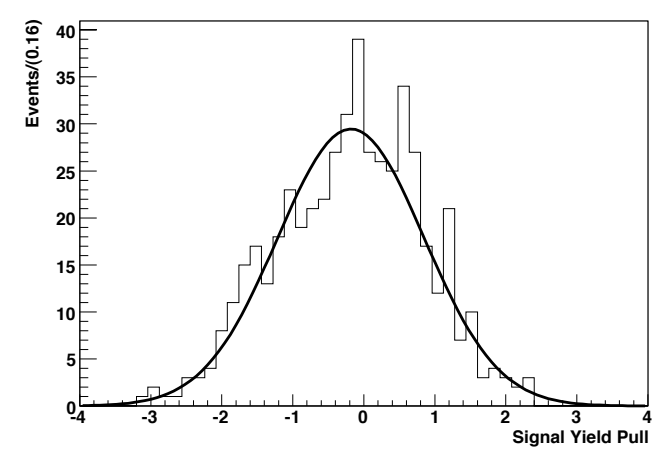

(a) Low mass pure toy signal yield pull.

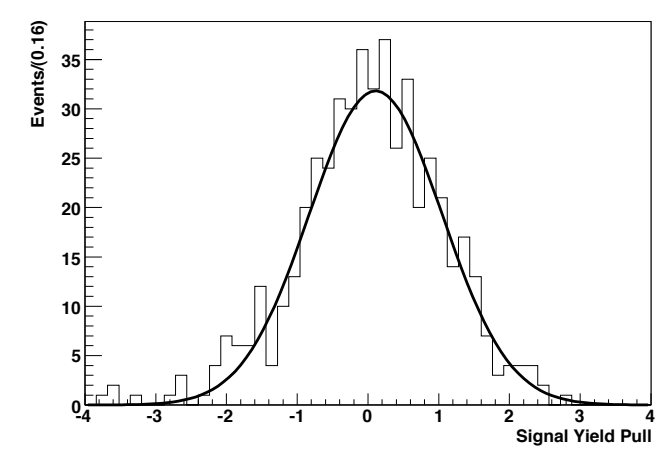

(c) Low mass embedded signal, $X_{d}$ cross-feed and $X_{s}$ cross-feed toy signal yield pull.

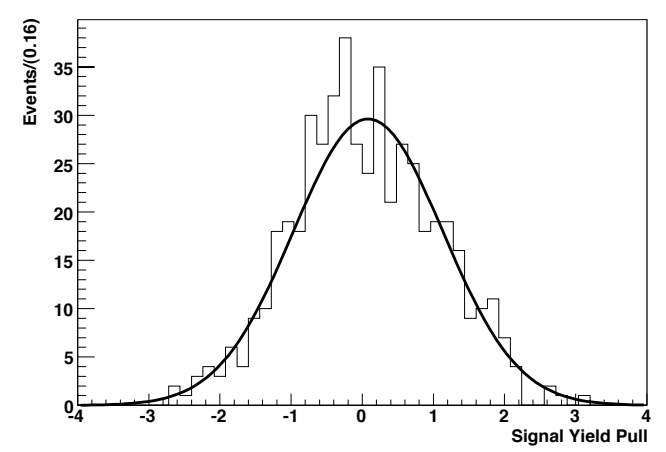

(e) High mass embedded signal and $X_{d}$ cross-feed toy signal yield pull.

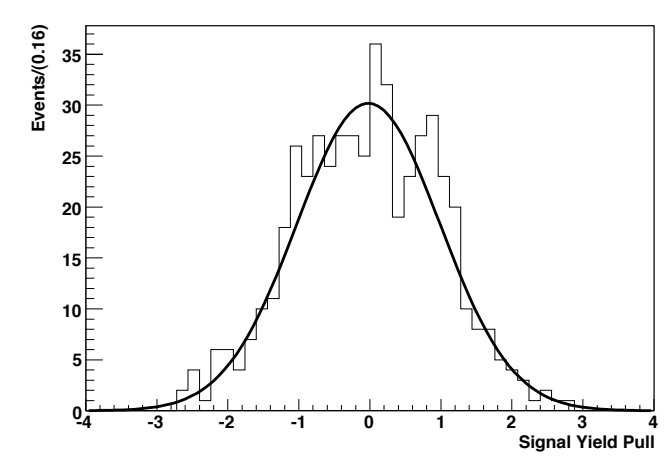

(b) Low mass embedded signal and $X_{d}$ cross-feed toy signal yield pull.

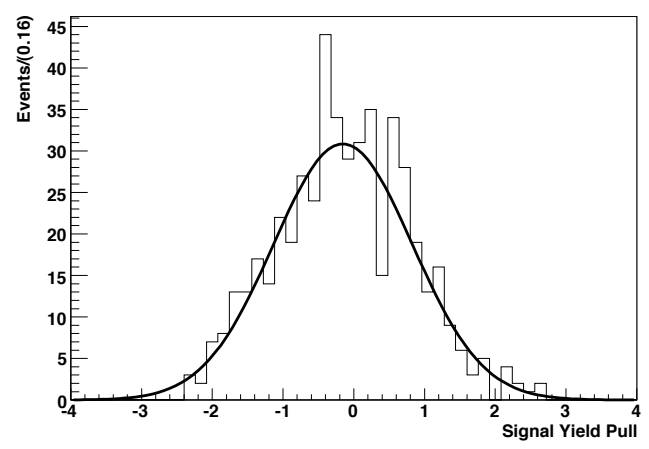

(d) High mass pure toy signal yield pull.

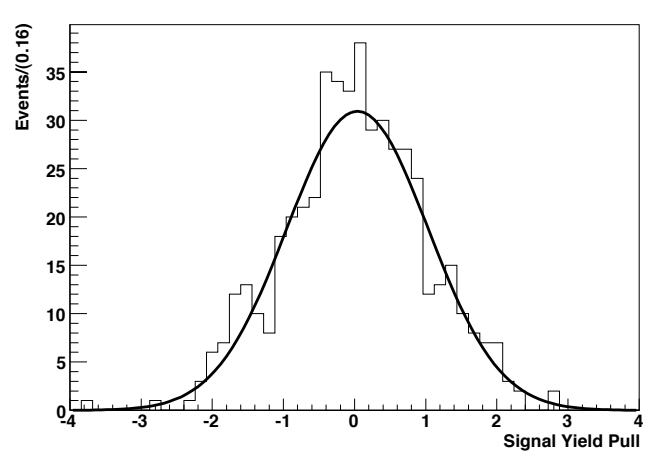

(f) High mass embedded signal, $X_{d}$ cross-feed and $X_{s}$ cross-feed toy signal yield pull.

Figure 4.14: Toy study signal yield pull histograms and fitted Gaussian distributions for $B \rightarrow X_{d} \gamma$ candidate fits to experimental data. Each study consists of 500 toy fits. 


\begin{tabular}{|c|c|c|c|c|c|}
\hline \multicolumn{2}{|c|}{ Parameter } & Pure Study & Embedded Study & Embedded $X_{s}$ Study & $\rho_{s i g}$ \\
\hline \multirow[t]{2}{*}{$n_{s i g}$} & $\mu$ & $-0.153 \pm 0.048$ & $0.082 \pm 0.052$ & $0.041 \pm 0.048$ & 1.000 \\
\hline & $\sigma$ & $0.983 \pm 0.041$ & $1.051 \pm 0.043$ & $0.995 \pm 0.043$ & \\
\hline \multirow[t]{2}{*}{$n_{\text {cont }}$} & $\mu$ & $0.036 \pm 0.047$ & $-0.068 \pm 0.045$ & $0.050 \pm 0.043$ & -0.222 \\
\hline & $\sigma$ & $0.997 \pm 0.038$ & $0.937 \pm 0.037$ & $0.893 \pm 0.036$ & \\
\hline \multirow[t]{2}{*}{$\xi$} & $\mu$ & $-0.104 \pm 0.050$ & $-0.027 \pm 0.046$ & $-0.097 \pm 0.046$ & 0.326 \\
\hline & $\sigma$ & $1.031 \pm 0.043$ & $0.986 \pm 0.037$ & $0.979 \pm 0.035$ & \\
\hline \multirow[t]{2}{*}{$a_{1}$} & $\mu$ & $0.057 \pm 0.044$ & $0.028 \pm 0.046$ & $-0.021 \pm 0.050$ & 0.026 \\
\hline & $\sigma$ & $0.907 \pm 0.035$ & $0.951 \pm 0.036$ & $1.031 \pm 0.043$ & \\
\hline
\end{tabular}

TABLE 4.10: Fitted Gaussian parameters to pull distributions from toy MC studies for all floated parameters in the high mass $B \rightarrow X_{d} \gamma$ fit. Also shown are the average correlation coefficients between each floated variable and $n_{\text {sig }}$ for the embedded toy study where both $X_{d}$ and $X_{s}$ MC data are embedded.

\subsection{Fits To Experimental Data}

The fits described above are applied to experimental data passing event selection cuts in each of the four event reconstruction categories. The results of these fits and their physical interpretation are presented here.

\subsection{1 $X_{s}$ Candidate Fit Results}

The $B \rightarrow X_{s} \gamma$ fits to experimental data converge after four complete iterations between the mass regions to correct cross-feed yields. Tables 4.11 and 4.12 list the initial, final and bias corrected values with associated errors for all variables floated in the fits to data in the low and high mass regions respectively. Bias corrections are applied by multiplying the average pull from the relevant toy study by the error returned from the experimental fit and then subtracting the resulting product from the fitted value for that variable. The tables also show the revised cross-feed yields after iteration. Figure 4.15 shows projection plots of the combined fit model and key contributing backgrounds for $m_{E S}^{\prime}$ and $\Delta E$ in the low mass region. The corresponding projection plots for the high mass region are shown in figure 4.16. The $m_{E S}^{\prime}$ projection plots are produced after applying a cut of $-0.15 \leq \Delta E \leq 0.1 \mathrm{GeV}$ and the $\Delta E$ projection plots are produced after applying a cut of $m_{E S}^{\prime} \geq 5.27 \mathrm{GeV} / c^{2}$. All projection plots show the fit model is in good agreement with the experimental data in both the high and low mass regions.

The low mass signal yield from experimental data is in reasonable agreement with the expected signal yield. The high mass signal yield is lower than the corresponding estimated value from MC studies. However, this does not account for systematic effects which are estimated in chapter 5 and so is not thought to be significant. 


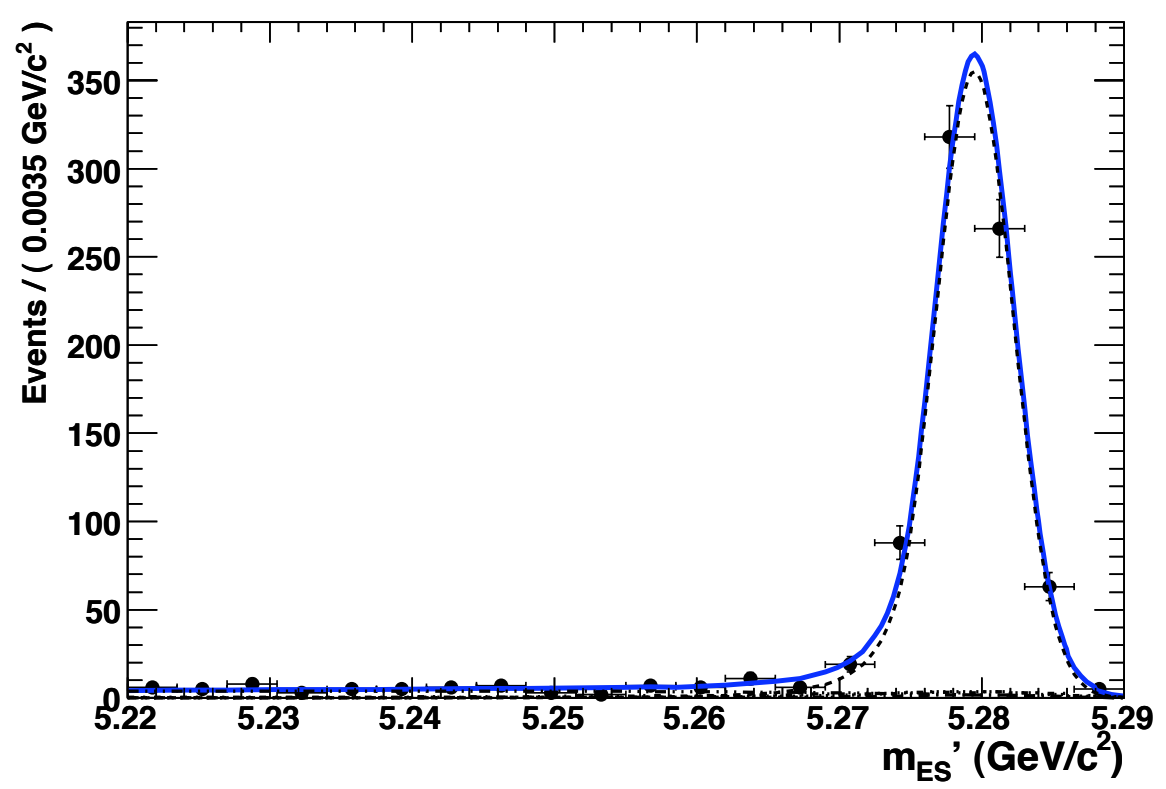

(a) $m_{E S}^{\prime}$ projection plot

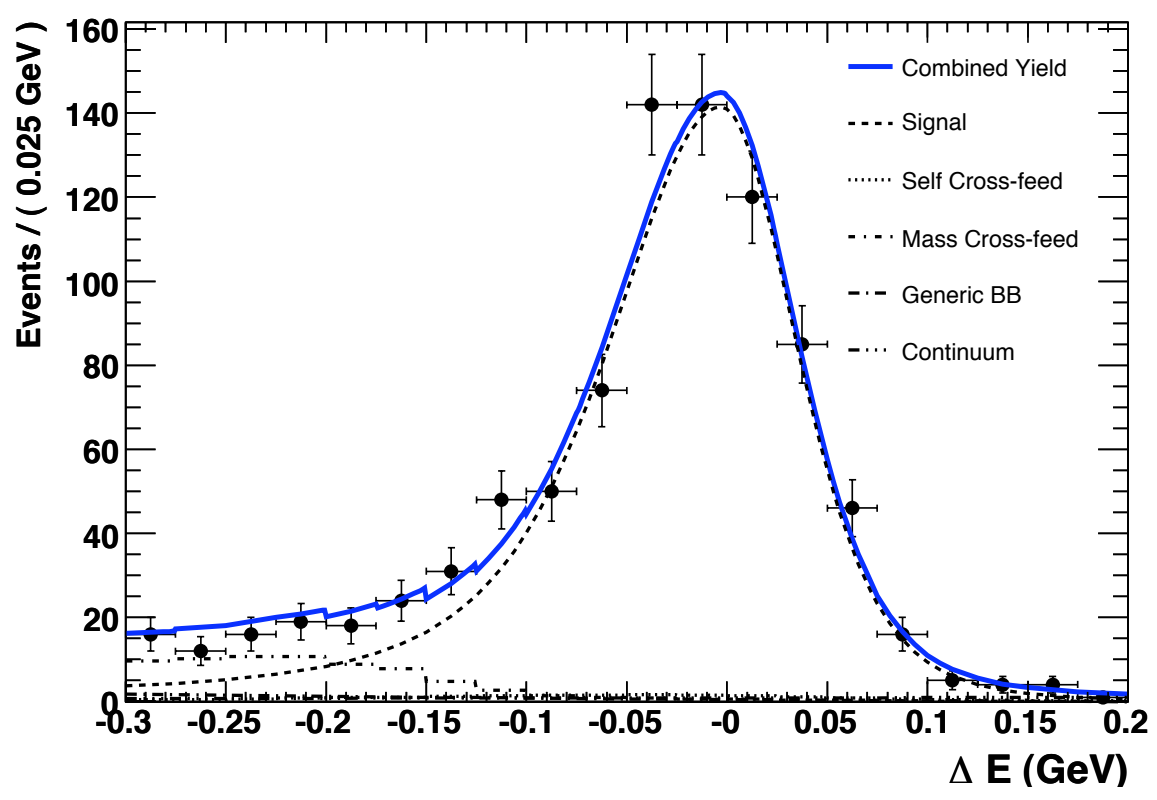

(b) $\Delta E$ projection plot

Figure 4.15: Projection plots of $m_{E S}^{\prime}$ and $\Delta E$ for $B \rightarrow X_{s}^{L} \gamma$ candidate fit to experimental data. The legend is applicable to both plots and indicates the relative contribution from each event hypothesis to the overall combined yield in experimental data. 


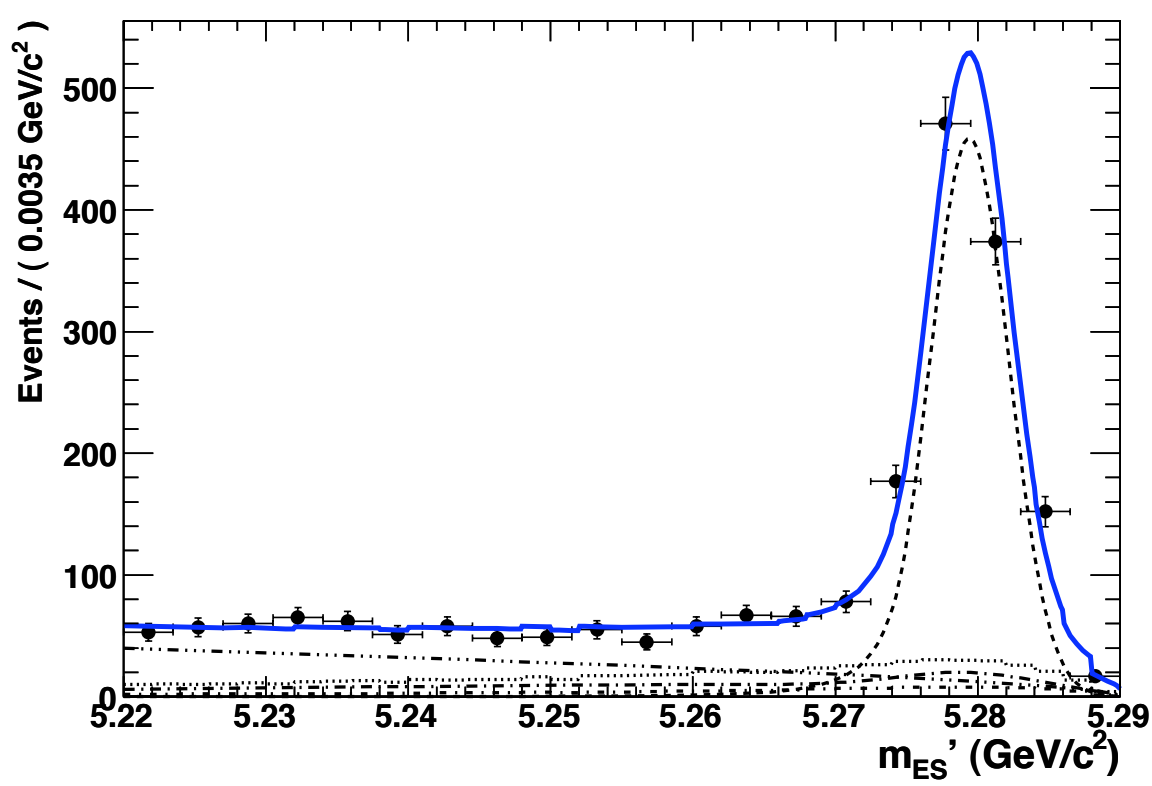

(a) $m_{E S}^{\prime}$ projection plot

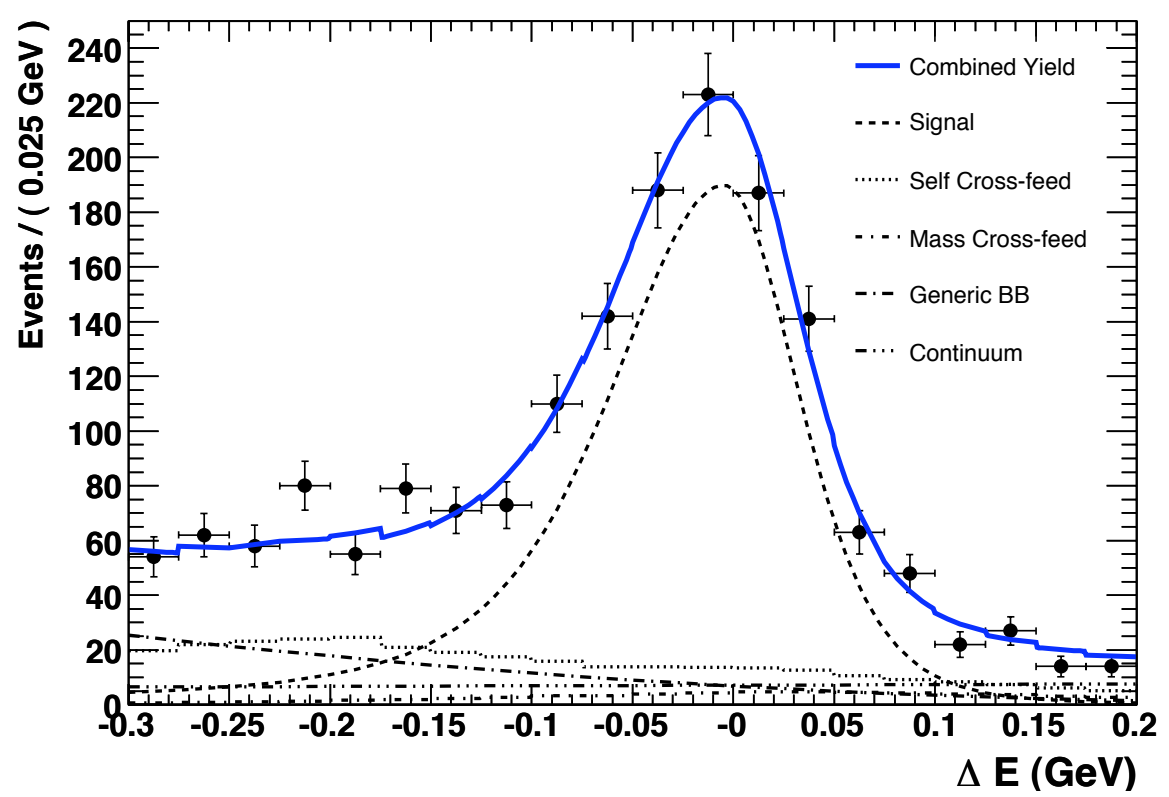

(b) $\Delta E$ projection plot

Figure 4.16: Projection plots of $m_{E S}^{\prime}$ and $\Delta E$ for $B \rightarrow X_{s}^{H} \gamma$ candidate fit to experimental data. The legend is applicable to both plots and indicates the relative contribution from each event hypothesis to the overall combined yield in experimental data. 


\begin{tabular}{|l|l|l|l|}
\hline Parameter & Initial Value & Final Value & Fit Bias Corrected Value \\
\hline$n_{\text {sig }}$ & 801 & $770 \pm 29$ & $774 \pm 29$ \\
$n_{\text {cont }}$ & 144 & $118 \pm 14$ & $119 \pm 14$ \\
$\sigma_{L}$ & $0.0688 \pm 0.0004$ & $0.0523 \pm 0.0029$ & $0.0536 \pm 0.0029$ \\
$\sigma_{R}$ & $0.0346 \pm 0.0003$ & $0.0365 \pm 0.0018$ & $0.0370 \pm 0.0018$ \\
$n_{\text {scf }}$ & 32 & 31 & - \\
$n_{m c f}$ & 155 & 149 & - \\
\hline
\end{tabular}

TABLE 4.11: Result of fit to experimental data for $B \rightarrow X_{s}^{L} \gamma$ candidates showing initial, final and bias-corrected values for variables floated in the fit as well as the corrected cross-feed yields after iteration.

\begin{tabular}{|l|l|l|l|}
\hline Parameter & Initial Value & Final Value & Fit Bias Corrected Value \\
\hline$n_{\text {sig }}$ & 1112 & $1016 \pm 39$ & $1015 \pm 39$ \\
$n_{\text {cont }}$ & 1113 & $996 \pm 51$ & $990 \pm 51$ \\
$\sigma_{L}$ & $0.0648 \pm 0.0008$ & $0.0532 \pm 0.0032$ & $0.0559 \pm 0.0032$ \\
$\sigma_{R}$ & $0.0341 \pm 0.0005$ & $0.0355 \pm 0.0019$ & $0.0361 \pm 0.0019$ \\
$\xi$ & $-9.3 \pm 9.3$ & $4.6 \pm 8.4$ & $4.6 \pm 8.4$ \\
$a_{1}$ & $0.47 \pm 0.16$ & $0.31 \pm 0.32$ & $0.35 \pm 0.32$ \\
$n_{\text {scf }}$ & 879 & 803 & - \\
$n_{m c f}$ & 155 & 149 & - \\
\hline
\end{tabular}

TABle 4.12: Result of fit to experimental data for $B \rightarrow X_{s}^{H} \gamma$ candidates showing initial, final and bias-corrected values for variables floated in the fit as well as the corrected cross-feed yields after iteration.

\subsection{2 $X_{d}$ Candidate Fit Results}

Before fitting for $B \rightarrow X_{d} \gamma$ candidates in experimental data the expected $X_{s}$ cross-feed yields and $\Delta E$ signal distribution widths are corrected using the results from fits to $B \rightarrow X_{s} \gamma$ candidates in experimental data. Table 4.13 lists the modified $X_{s}$ cross-feed component yields. They are corrected by applying the ratio of corresponding measured and estimated signal yield obtained from the $B \rightarrow X_{s} \gamma$ candidate fits. Table 4.14 lists the modified $\Delta E$ signal width parameters, $\sigma_{X d}^{\prime}$, given by,

$$
\sigma_{X d}^{\prime}=\sigma_{X d}^{M C} \frac{\sigma_{X s}^{d a t a}}{\sigma_{X s}^{M C}}
$$

where $\sigma_{X d}^{M C}$ is the corresponding fitted width in $B \rightarrow X_{d} \gamma$ candidate signal MC, $\sigma_{X s}^{\text {data }}$ is the bias corrected width measured in $B \rightarrow X_{s} \gamma$ candidate experimental data and $\sigma_{X s}^{M C}$ is the width from fitting $B \rightarrow X_{s} \gamma$ candidate signal MC data. The associated error for $\sigma_{X d}^{\prime}$ is obtained from,

$$
\epsilon=\sigma_{X d}^{\prime} \frac{\epsilon_{X s}^{\text {data }}}{\sigma_{X s}^{\text {data }}}
$$


(a) Low mass $B \rightarrow X_{d} \gamma$ candidate yields.

\begin{tabular}{|l|l|l|}
\hline Yield & Initial & Corrected \\
\hline$n_{P I D}^{X s}$ & 13 & 13 \\
$n_{s c f}^{X s}$ & 22 & 21 \\
$n_{m c f}^{X s}$ & 36 & 33 \\
\hline
\end{tabular}

(b) High mass $B \rightarrow X_{d} \gamma$ candidate yields.

\begin{tabular}{|l|l|l|}
\hline Yield & Initial & Corrected \\
\hline$n_{P I D}^{X s}$ & 11 & 10 \\
$n_{s c f}^{X s}$ & 324 & 295 \\
$n_{m c f}^{X s}$ & 18 & 17 \\
\hline
\end{tabular}

TABLE 4.13: Corrections of $B \rightarrow X_{s} \gamma$ cross-feed component yields in (a) the low mass and (b) the high mass fit to $B \rightarrow X_{d} \gamma$ candidates after fitting for $B \rightarrow X_{s} \gamma$ candidates in experimental data.

(a) Low mass $B \rightarrow X_{d} \gamma$ fit.

\begin{tabular}{|l|l|l|}
\hline Width & Initial & Corrected \\
\hline$\sigma_{L}$ & $0.0726 \pm 0.0011$ & $0.0566 \pm 0.0031$ \\
$\sigma_{R}$ & $0.0367 \pm 0.0007$ & $0.0392 \pm 0.0019$ \\
\hline
\end{tabular}

(b) High mass $B \rightarrow X_{d} \gamma$ fit.

\begin{tabular}{|l|l|l|}
\hline Width & Initial & Corrected \\
\hline$\sigma_{L}$ & $0.0684 \pm 0.0026$ & $0.0590 \pm 0.0034$ \\
$\sigma_{R}$ & $0.0352 \pm 0.0016$ & $0.0373 \pm 0.0020$ \\
\hline
\end{tabular}

TABLE 4.14: Corrections to $\Delta E$ signal widths in (a) the low mass and (b) the high mass fit to $B \rightarrow X_{d} \gamma$ candidates after fitting for $B \rightarrow X_{s} \gamma$ candidates in experimental data.

where $\epsilon_{X s}^{\text {data }}$ is the associated error from the fit to $B \rightarrow X_{s} \gamma$ candidate experimental data.

The $B \rightarrow X_{d} \gamma$ fits to experimental data converge after three complete iterations between the mass regions to correct cross-feed yields. Tables 4.15 and 4.16 list the initial, final and bias corrected values with associated errors for all variables floated in the fits to data in the low and high mass regions respectively. Also shown are the revised cross-feed yields after iteration. Figure 4.17 shows projection plots of the combined fit model and key contributing backgrounds for $m_{E S}^{\prime}$ and $\Delta E$ in the low mass region. The corresponding projection plots for the high mass region are shown in figure 4.16. The $m_{E S}^{\prime}$ projection plots are produced after applying a cut of $-0.15 \leq \Delta E \leq 0.1 \mathrm{GeV}$ and the $\Delta E$ projection plots are produced after applying a cut of $m_{E S}^{\prime} \geq 5.27 \mathrm{GeV} / c^{2}$. All projection plots show the fit model is in good agreement with the experimental data in both the high and low mass regions.

The low mass signal yield in experimental data is in good agreement with the expected yield. For the high mass signal yield an enhancement compared to MC data estimates is observed. However, these estimates use an order of magnitude calculation for the expected inclusive $B \rightarrow X_{d} \gamma$ BF. It follows that the initial and expected values should 


\begin{tabular}{|l|l|l|l|}
\hline Parameter & Initial Value & Final Value & Fit Bias Corrected Value \\
\hline$n_{\text {sig }}$ & 35 & $34 \pm 9$ & $33 \pm 9$ \\
$n_{\text {cont }}$ & 404 & $366 \pm 22$ & $367 \pm 22$ \\
$n_{\text {scf }}$ & 3 & 3 & - \\
$n_{m c f}$ & 7 & 12 & - \\
\hline
\end{tabular}

TABle 4.15: Result of fit to experimental data for $B \rightarrow X_{d}^{L} \gamma$ candidates showing initial, final and bias-corrected values for variables floated in the fit as well as the corrected cross-feed yields after iteration.

\begin{tabular}{|l|l|l|l|}
\hline Parameter & Initial Value & Final Value & Fit Bias Corrected Value \\
\hline$n_{\text {sig }}$ & 37 & $60 \pm 15$ & $59 \pm 15$ \\
$n_{\text {cont }}$ & 1722 & $1450 \pm 49$ & $1448 \pm 49$ \\
$\xi$ & $-19.8 \pm 2.9$ & $-5.0 \pm 5.3$ & $-4.5 \pm 5.3$ \\
$a_{1}$ & $-0.29 \pm 0.14$ & $-0.12 \pm 0.22$ & $-0.12 \pm 0.22$ \\
$n_{\text {scf }}$ & 37 & 60 & - \\
$n_{m c f}$ & 8 & 8 & - \\
\hline
\end{tabular}

TABle 4.16: Result of fit to experimental data for $B \rightarrow X_{d}^{H} \gamma$ candidates showing initial, final and bias-corrected values for variables floated in the fit as well as the corrected cross-feed yields after iteration.

therefore only be expected to agree in order of magnitude and this is indeed the case. The statistical significance of this measurement does improve significantly on the previous BABAR measurement [1] and represents the first evidence for $B \rightarrow X_{d} \gamma$ transitions in the hadronic mass region above the $\rho$ and $\omega$ resonances.

\subsubsection{Quality of Fits to Experimental Data}

Agreement between each combined yield PDF distribution and corresponding experimental data is evaluated numerically using a chi square goodness of fit test [63, 64]. Each 2D combined yield PDF is integrated numerically in a grid of 20 bins in $\Delta E$ and 20 bins $^{5}$ in $m_{E S}^{\prime}$ for this calculation. The integrated grid is then projected in each variable with a cut placed on the signal region of the orthogonal variable, thus emulating the projected PDF distributions shown above as binned histograms. The corresponding experimental data is projected identically for each variable. The chi square statistic comparing the PDF and experimental data histograms is given by,

$$
\chi^{2}=\sum_{k} \frac{\left(n_{P D F}^{k}-n_{\text {Data }}^{k}\right)^{2}}{\sigma_{\text {Data }}^{2}},
$$

\footnotetext{
${ }^{5}$ The binning in $m_{E S}^{\prime}$ is reduced from 35 bin to 20 bins for this calculation to ensure sufficient statistics per bin in the projection of this variable. In particular in the $B \rightarrow X_{d}^{L} \gamma$ fit has relatively few experimental data events.
} 


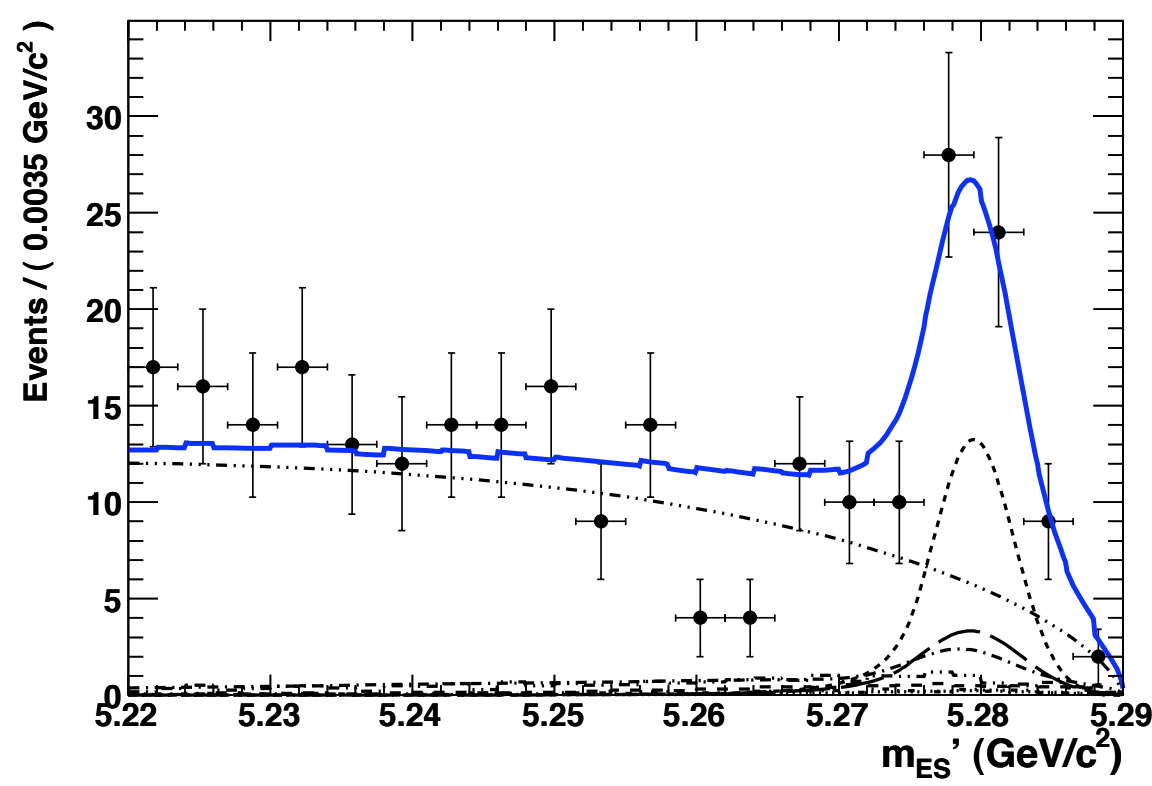

(a) $m_{E S}^{\prime}$ projection plot

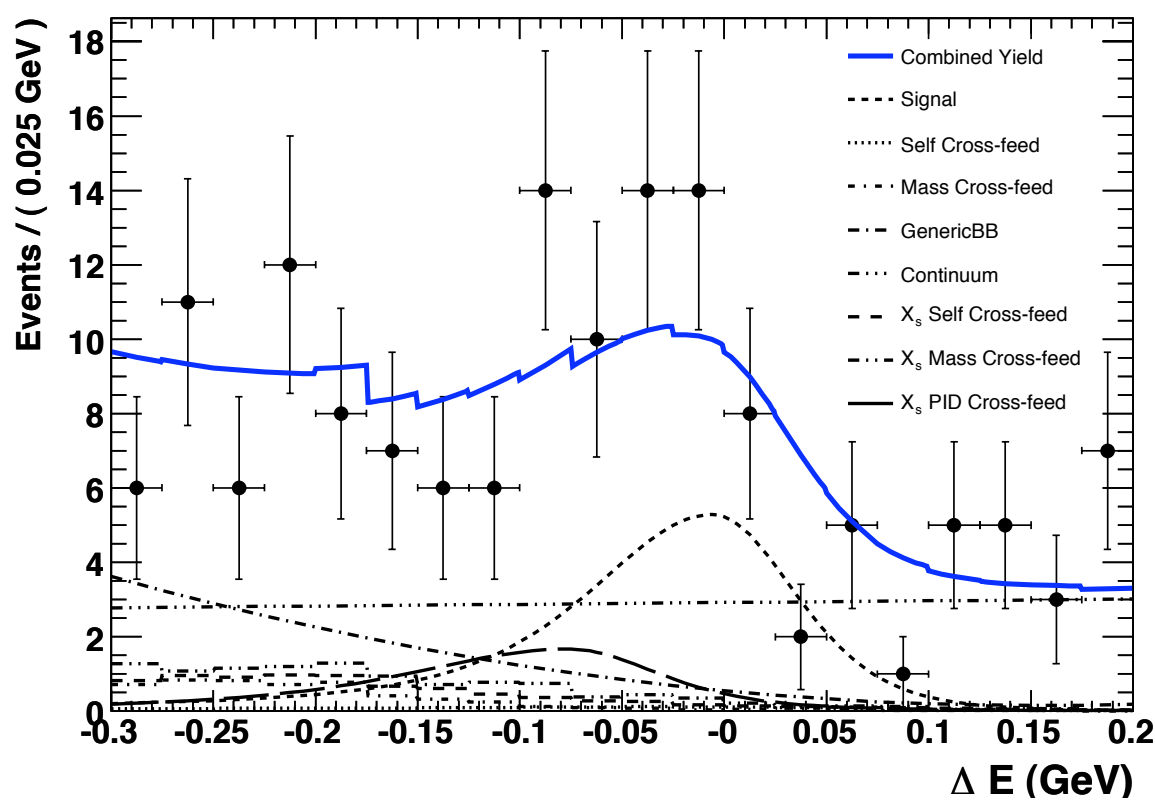

(b) $\Delta E$ projection plot

Figure 4.17: Projection plots of $m_{E S}^{\prime}$ and $\Delta E$ for $B \rightarrow X_{d}^{L} \gamma$ candidate fit to experimental data. The legend is applicable to both plots and indicates the relative contribution from each event hypothesis to the overall combined yield in experimental data. 


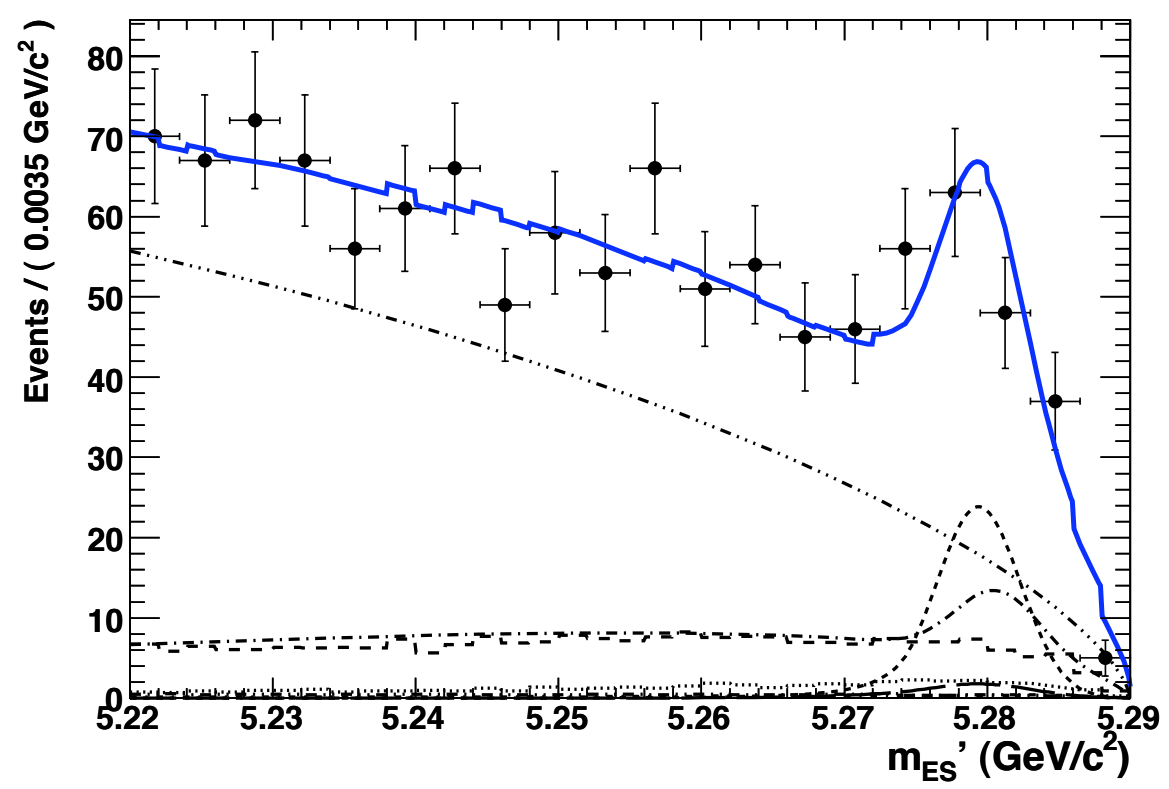

(a) $m_{E S}^{\prime}$ projection plot

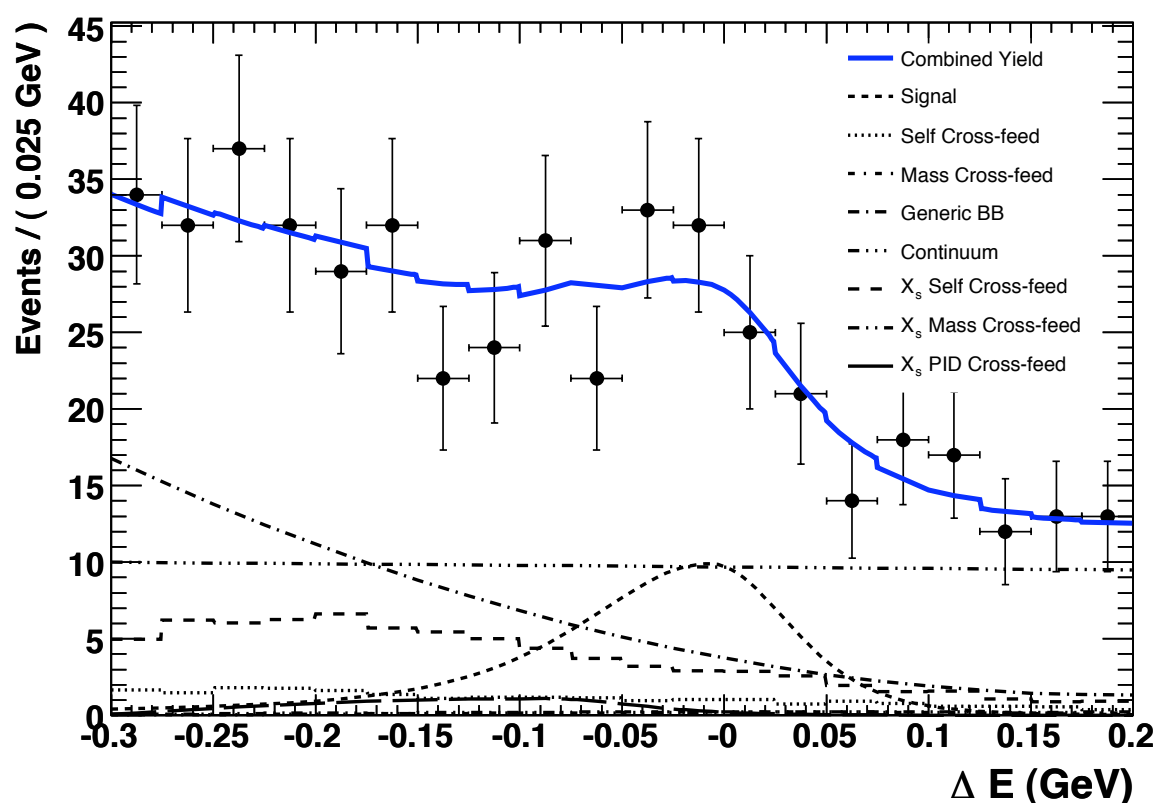

(b) $\Delta E$ projection plot

Figure 4.18: Projection plots of $m_{E S}^{\prime}$ and $\Delta E$ for $B \rightarrow X_{d}^{H} \gamma$ candidate fit to experimental data. The legend is applicable to both plots and indicates the relative contribution from each event hypothesis to the overall combined yield in experimental data. 


\begin{tabular}{|l|l|l|l|}
\hline Fit & Variable & $\chi_{\text {calc }}^{2}$ & $\mathcal{P}\left(\chi^{2}>\chi_{\text {calc }}^{2}\right)$ \\
\hline$B \rightarrow X_{s}^{L} \gamma$ & $m_{E S}$ & 15.153 & 0.713 \\
& $\Delta E$ & 14.669 & 0.743 \\
\hline$B \rightarrow X_{s}^{H} \gamma$ & $m_{E S}$ & 9.664 & 0.961 \\
& $\Delta E$ & 17.7486 & 0.539 \\
\hline$B \rightarrow X_{d}^{L} \gamma$ & $m_{E S}$ & 13.308 & 0.822 \\
& $\Delta E$ & 16.760 & 0.606 \\
\hline$B \rightarrow X_{d}^{H} \gamma$ & $m_{E S}$ & 16.188 & 0.645 \\
& $\Delta E$ & 6.276 & 0.997 \\
\hline
\end{tabular}

TABLE 4.17: $\chi^{2}$ goodness of fit tests for fits to experimental data. Each test has 19 DOF and the probability of obtaining a $\chi^{2}$ value greater than calculated given that many DOF is shown.

where $n_{P D F}^{k}$ and $n_{\text {Data }}^{k}$ are the respective projections for bin $k$ and $\sigma_{D a t a}$ is the positive (negative) Poisson error on the number of data events in that bin if $\left(n_{P D F}^{k}-n_{\text {Data }}^{k}\right)$ is positive (negative). Given a calculated chi square value, $\chi_{\text {calc }}^{2}$, and the number of degrees of freedom (DOF) in the calculation, which here is 19 for all cases, it is possible to calculate the probability that the $\chi^{2}$ value would exceed $\chi_{\text {calc }}^{2}$ in an independent repetition of the test $[63,64]$. This is to a good approximation the probability that the agreement between model and data will be worse if the analysis were repeated on an independent dataset. Table 4.17 lists the $\chi^{2}$ value calculated for each of the eight data projections and associated probability. It can be seen the probabilities are all in excess of 0.5 suggesting that the models are in all cases good representations of the data.

\subsubsection{Interpretation of Results}

The above signal yields are converted into measured BFs through the relation,

$$
\sum_{i=1}^{7} \mathcal{B}\left(B \rightarrow X_{q}^{i, m} \gamma\right)=\frac{n_{\text {sig }}}{2 n_{B \bar{B}} \epsilon}
$$

where $i$ is the mode index, $q=s, d$ identifies the decay flavour, $m=L, H$ identifies the mass region, $n_{\text {sig }}$ is the signal yield from the fit, $n_{B \bar{B}}$ is the total number of $B \bar{B}$ pairs in data (470.9 million $B \bar{B}$ pairs) and $\epsilon$ is the signal event selection efficiency calculated from MC data. Signal efficiencies are calculated by taking the ratio of signal events surviving cuts in a given mass range to the total number of signal events generated with true hadronic mass in that mass range. Table 4.18 lists the signal yields, calculated efficiencies and estimated measured BFs with associated statistical errors. 


\begin{tabular}{|l|l|l|l|}
\hline Fit & Yield & MC Efficiency & Measured BF \\
\hline$B \rightarrow X_{s}^{L} \gamma$ & $774 \pm 29$ & 0.0447 & $(1.84 \pm 0.07) \times 10^{-5}$ \\
$B \rightarrow X_{s}^{H} \gamma$ & $1015 \pm 39$ & 0.0171 & $(6.30 \pm 0.24) \times 10^{-5}$ \\
$B \rightarrow X_{d}^{L} \gamma$ & $33 \pm 9$ & 0.0361 & $(0.97 \pm 0.27) \times 10^{-6}$ \\
$B \rightarrow X_{d}^{H} \gamma$ & $59 \pm 15$ & 0.0174 & $(3.60 \pm 0.92) \times 10^{-6}$ \\
\hline
\end{tabular}

TABLE 4.18: Measured BF estimation and associated statistical error for each fit to experimental data. 


\section{Chapter 5}

\section{Post Fit Studies}

The results from fits to experimental data are interpreted as BF measurements. This chapter describes the estimation of systematic uncertainties for each measured $\mathrm{BF}$ and then details the model-dependent extrapolation studies using MC data to estimate the inclusive BFs of both $B \rightarrow X_{s, d} \gamma$. Finally the ratio of these inclusive fractions is used to estimate a value of $\left|V_{t d} / V_{t s}\right|$.

\subsection{Experimental Systematic Uncertainties}

Systematic uncertainties from the experimental techniques described in chapters 3 and 4 can be divided into five categories: Firstly differences in the reconstruction efficiency of primary particles within the BABAR framework between MC and experimental data; secondly differences in efficiencies between MC and experimental data of the cuts made during event reduction; thirdly uncertainties arising from fixing PDF parameters and event hypothesis yields in the MLF to experimental data; fourthly uncertainties in the models used to generate signal MC data, in particular the KN photon energy spectrum and JETSET phase space decay distribution of hadronic final states; finally the uncertainty in the estimate of the total number of $B \bar{B}$ pair events in experimental data. The error for this last category is given in section 3.1 .1 and corresponds to a $0.6 \%$ uncertainty on each measured BF.

\subsubsection{Reconstruction Efficiencies}

Lists of reconstructed charged tracks and neutral clusters accessed from the central event store have different reconstruction efficiencies in $\mathrm{MC}$ and experimental data. This is because the GEANT4 simulation of the BABAR detector does not always accurately model 


\begin{tabular}{|l|l|l|}
\hline Fit Class & $\mu_{t r k}$ & $\mu_{t r k} \sigma_{t r k}$ \\
\hline$B \rightarrow X_{s}^{L} \gamma$ & 1.43 & $0.30 \%$ \\
$B \rightarrow X_{s}^{H} \gamma$ & 2.31 & $0.49 \%$ \\
$B \rightarrow X_{d}^{L} \gamma$ & 1.57 & $0.33 \%$ \\
$B \rightarrow X_{d}^{H} \gamma$ & 1.93 & $0.41 \%$ \\
\hline
\end{tabular}

TABLE 5.1: Estimates of the charged track reconstruction systematic uncertainty for each experimental data fit.

the response of sub-detectors used in the reconstruction of those particle interactions. Dedicated groups within the BABAR collaboration study these differences with $\mathrm{MC}$ and experimental data control samples and advise analysts what uncertainty should be applied. The following summarises how the results of these dedicated BABAR studies have been applied to the reconstructed signal states in this analysis.

\subsubsection{Charged Tracks}

Differences between the reconstruction efficiencies of charged tracks in MC and experimental data are determined from a dedicated study carried out by the BABAR tracking group. The analysis is described in [68] and uses $\tau$ pair decays, where one $\tau$ decays leptonically and the subsequent charged lepton identifies the event. The recoiling $\tau$ must have an odd number of charged daughter particles. The study uses events where the recoiling $\tau$ has three charged daughters, two of which are charged pions either from a $\rho^{0} \rightarrow \pi^{+} \pi^{-}$decay or where both pions have total invariant mass greater than $300 \mathrm{MeV} / c^{2}$ and opposite charge to the lepton from the other $\tau$ decay. These two pions and the charged lepton are identified in event reconstruction through strict PID, geometrical and kinematic criteria. The remaining charged daughter is either detected or not and the difference in its detection efficiency between $\mathrm{MC}$ and experimental data is assigned as the systematic uncertainty for charged track reconstruction. To account for the average multiplicity of these events being lower than that of a $B \bar{B}$ event a further correction factor is applied to the systematic uncertainty. For the datasets and reconstruction framework software used in this analysis the BABAR tracking group advise assigning an uncertainty of $\sigma_{t r k}=0.21 \%$ per track from the GoodTracksLoose list after the multiplicity correction is applied. For each of the fits described in chapter 4 the product of this uncertainty with the mean track multiplicity of correctly reconstructed MC signal decays after all event reduction cuts, $\mu_{t r k}$, is therefore applied as the total systematic uncertainty due to track reconstruction. These are summarised in table 5.1. 


\begin{tabular}{|l|l|l|l|l|}
\hline & $B \rightarrow X_{s}^{L} \gamma$ & $B \rightarrow X_{s}^{H} \gamma$ & $B \rightarrow X_{d}^{L} \gamma$ & $B \rightarrow X_{d}^{H} \gamma$ \\
\hline \hline$\mu_{\pi^{0}}$ & 0.29 & 0.30 & 0.45 & 0.48 \\
$\mu_{\pi^{0}} \sigma_{\pi^{0}}$ & $0.9 \%$ & $0.9 \%$ & $1.3 \%$ & $1.4 \%$ \\
\hline$\mu_{\eta}$ & - & 0.04 & - & 0.03 \\
$\mu_{\eta} \sigma_{\eta}$ & - & $0.1 \%$ & - & $0.1 \%$ \\
\hline$\sigma_{\gamma}$ & $0.7 \%$ & $0.7 \%$ & $0.7 \%$ & $0.7 \%$ \\
\hline \hline$\sigma_{\text {neu }}$ & $1.6 \%$ & $1.7 \%$ & $2.0 \%$ & $2.2 \%$ \\
\hline
\end{tabular}

TABLE 5.2: Estimates of the neutral particle reconstruction systematic uncertainty for each experimental data fit.

\subsubsection{Neutral Particles}

Studies performed by the BABAR neutral particles group are used to determine reconstruction efficiency differences in MC and experimental data for lists of photons as well as reconstructed $\pi^{0}$ and $\eta$ mesons. The analysis described in [69] uses control samples of $\tau \rightarrow \pi \nu$ and $\tau \rightarrow \rho \nu$ decays to compare the reconstruction efficiencies of $\pi^{0}$ mesons and single photons. Consequently the BABAR neutral group recommend assigning a systematic uncertainty of $\sigma_{\pi^{0}}=3.0 \%$ per $\pi^{0}$ from the PiODefaultMass list and $1.8 \%$ per single photon from the GoodPhotonLoose list, therefore giving an uncertainty per $\eta$ meson of $\sigma_{\eta}=3.6 \%$, where $\eta \rightarrow \gamma \gamma$. The study described in [70] compares samples of $e^{+} e^{-} \rightarrow \mu^{+} \mu^{-} \gamma$ events in MC and experimental data to determine differences in reconstruction efficiency of high energy photons. Using the results of this study the BABAR neutral group advise assigning an uncertainty of $\sigma_{\gamma}=0.7 \%$ for photons with a laboratory frame energy greater than $1 \mathrm{GeV}$.

Table 5.2 summarises the total systematic uncertainty, $\sigma_{n e u}$, calculated for neutral particle reconstruction in each of the fits described in chapter 4 . The $\pi^{0}$ and $\eta$ uncertainties are calculated respectively as $\mu_{\pi^{0}} \sigma_{\pi^{0}}$ and $\mu_{\eta} \sigma_{\eta}$, where $\mu_{M}$ is the mean multiplicity of meson $M$ in correctly reconstructed signal $\mathrm{MC}$ decays after event reduction cuts. As all neutral particle reconstruction uncertainties arise from photon detection in the EMC they are $100 \%$ correlated and therefore the total systematic uncertainty is a linear combination of those due to individual particles.

\subsubsection{Event Reduction Cut Efficiencies}

Imperfections in the detector simulation can lead to differences in distributions of variables between MC and experimental data. In particular any difference in a variable distribution on which a cut is applied during event reconstruction and subsequent reduction can lead to a systematic difference in the signal efficiency estimated from MC 
data and the true cut efficiency in experimental data. The calculated BFs assume the MC efficiency accurately describes the data so an uncertainty must be applied for each variable which may negate this assumption. All variables identified as a potential source of uncertainty are listed below and their corresponding systematic uncertainty estimated.

\subsubsection{Photon Cuts}

The reduction cuts placed on the high energy photon of reconstructed candidates are the $\pi^{0} / \eta$ candidate veto, variables relating to EMC cluster quality, specifically the second moment and lateral moment and the cut on distance to the nearest track-matched cluster. Previous radiative penguin analyses using BABAR data have made extensive studies of such cuts and estimated the relative uncertainties which should be applied for each. This analysis does not significantly modify the implementation of these cuts so none of these studies are repeated. Instead the systematic uncertainties calculated in previous analyses are applied.

The analysis described in [71] investigates differences in performing the $\pi^{0} / \eta$ candidate veto in $\mathrm{MC}$ and experimental data. A simulated signal photon with energy $2.5 \mathrm{GeV}$ in the CM frame is embedded into different types of background MC and experimental data samples and the efficiency of the veto compared. The corresponding efficiencies are found to agree within $1 \%$ for each veto and hence this value is assigned as a systematic error. The errors are added linearly giving a total systematic uncertainty of $2 \%$ for these cuts. The same analysis also studies the effect of the track distance cut by embedding photon clusters extracted from $e^{+} e^{-} \rightarrow e^{+} e^{-} \gamma$ events into generic $B \bar{B}$ MC data and experimental data. An efficiency difference of $2 \%$ in applying the distance cut on these two samples is observed and applied as a systematic uncertainty.

The analysis described in [59] investigates photon cluster quality variables by comparing control samples of $e^{+} e^{-} \rightarrow \mu^{+} \mu^{-} \gamma$ decays in MC and experimental data. Only the second moment cut is found to show an efficiency difference, the magnitude of which is assigned as a systematic error of $2.1 \%$. The cluster quality cut uncertainty is added in quadrature with the distance cut uncertainty to give an combined photon quality systematic error of $2.9 \%$.

\subsubsection{Charged Track PID}

The BABAR PID group perform studies to identify differences in PID selector efficiencies between MC and experimental data as a function of track momentum. These studies use MC data events, from the control samples described in section 3.2.5, not used to 
train the classifiers and their experimental data equivalents. Figure 5.1 shows the results of these efficiency studies for the PID selectors used to identify signal candidates during event reduction, pionKMTight and kaonBDTNotAPion. For this analysis signal candidates are generally reconstructed from charged tracks with momentum less than $2.5 \mathrm{GeV} / c$ in the laboratory frame. The associated average systematic uncertainty for each selector are therefore taken from these plots to be $1.5 \%$ and $0.5 \%$ for pionKMTight and kaonBDTNotAPion respectively. Corrections for potential differences in event topology and track qualities between signal events and those used in the above control samples are not provided by the PID group; however, they advise that these are unlikely to be less than $1 \%$. Consequently an additional uncertainty of $1 \%$ is added in quadrature for each selector giving an overall uncertainty per track of $\sigma_{\pi}=1.8 \%$ and $\sigma_{K}=1.1 \%$ for pionKMTight and kaonBDTNotAPion respectively.
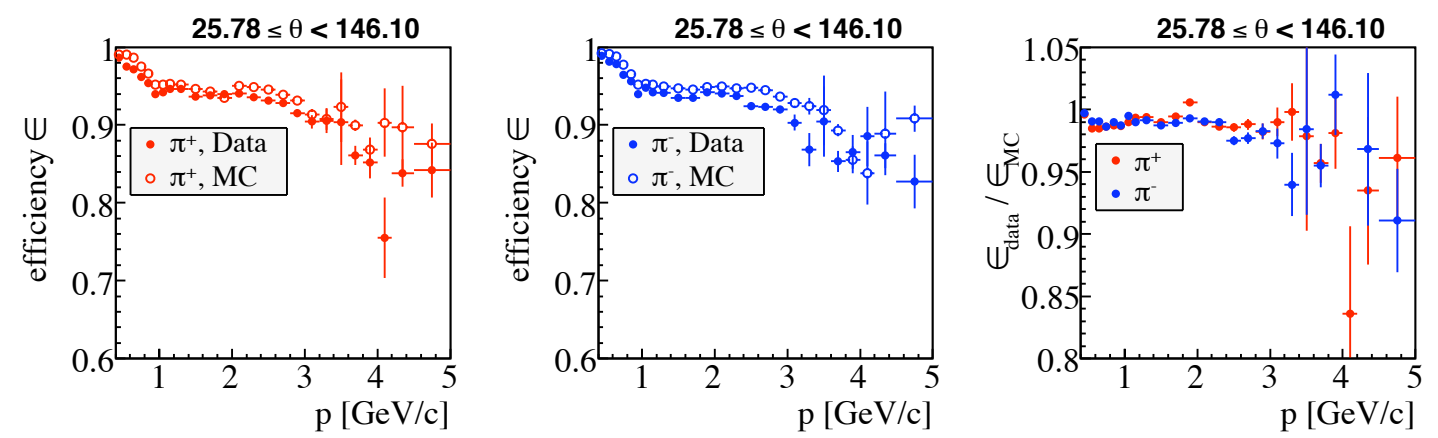

(a) Efficiency comparisons for true pions passing the pionKMTight selector. From the plot of $\epsilon_{d a t a} / \epsilon_{M C}$ an average systematic uncertainty of $1.5 \%$ per pion with momentum up to $2.5 \mathrm{GeV} / \mathrm{c}$ is assigned.
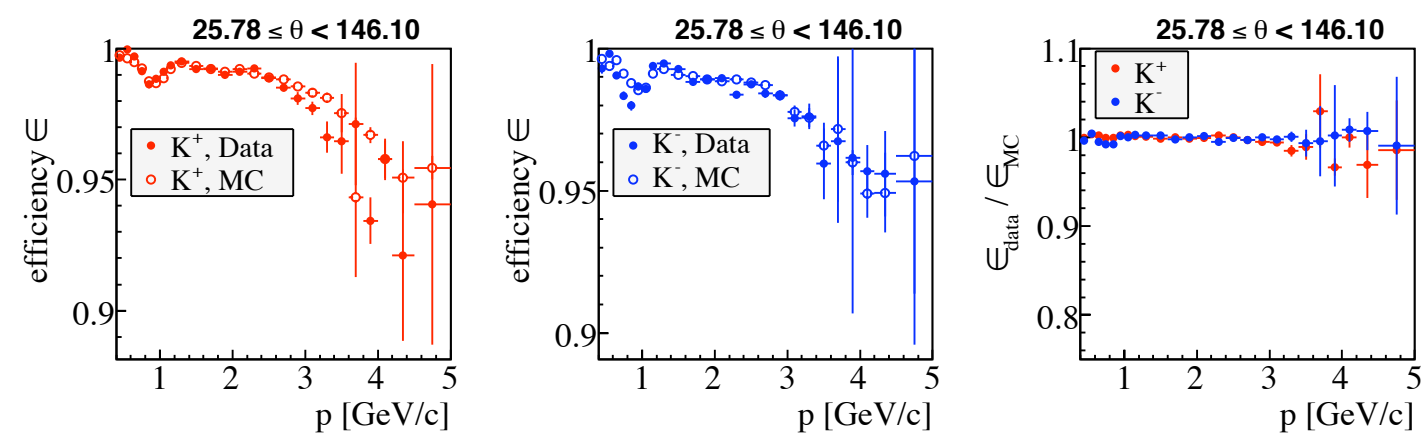

(b) Efficiency comparisons for true kaons passing the kaonBDTNotAPion selector. From the plot of $\epsilon_{\text {data }} / \epsilon_{M C}$ an average systematic uncertainty of $0.5 \%$ per kaon with momentum up to $2.5 \mathrm{GeV} / c$ is assigned.

Figure 5.1: Plots from the BABAR PID group showing efficiency comparisons of PID selector performance between MC and experimental data control samples for (a) pionKMTight and (b) kaonBDTNotAPion.

Signal candidates reconstructed in this analysis have PID selector requirements imposed on all charged tracks used in their reconstruction. Kaon candidates must pass the kaonBDTNotAPion selector and fail the pionKMLoose selector. Pion candidates must pass the pionKMTight selector and fail the kaonBDTNotAPion selector. Charged tracks 


\begin{tabular}{|l|l|l|l|l|}
\hline & $B \rightarrow X_{s}^{L} \gamma$ & $B \rightarrow X_{s}^{H} \gamma$ & $B \rightarrow X_{d}^{L} \gamma$ & $B \rightarrow X_{d}^{H} \gamma$ \\
\hline \hline$\mu_{\pi}$ & 0.71 & 1.31 & 1.57 & 1.93 \\
$\mu_{\pi} \sigma_{\pi}$ & $1.3 \%$ & $2.4 \%$ & $2.8 \%$ & $3.5 \%$ \\
\hline$\mu_{K}$ & 1.00 & 1.00 & - & - \\
$\mu_{K} \sigma_{K}$ & $1.1 \%$ & $1.1 \%$ & - & - \\
\hline \hline$\sigma_{P I D}$ & $2.4 \%$ & $3.5 \%$ & $2.8 \%$ & $3.5 \%$ \\
\hline
\end{tabular}

TABLE 5.3: Estimates of the PID selector systematic uncertainty for each experimental data fit.

passing the pionKMTight selector are a subset of those passing the pionKMLoose selector. One track in $10^{6}$ from the GoodTracksLoose list is found to pass both the pionKMTight and kaonBDTNotAPion in both signal MC and experimental data. For the purpose of applying a PID systematic uncertainty it is therefore assumed that the selectors are essentially mutually exclusive and that any track which fails one of these selectors due to PID inefficiency will not simultaneously be accepted by the other selector. Consequently the pion selector uncertainty need only be applied to tracks identified as pions and the kaon selector uncertainty only to tracks identified as kaons. There is significant overlap in many of the input variables used in each selector so a $100 \%$ correlation between the pion and kaon selector inefficiencies are assumed. The total PID systematic uncertainty for a class of signal events, $\sigma_{P I D}$, is therefore taken to be,

$$
\sigma_{P I D}=\sigma_{\pi} \mu_{\pi}+\sigma_{K} \mu_{K}
$$

where $\mu_{\pi}$ and $\mu_{K}$ are the mean number of respective charged pions and kaons in signal MC passing all event reduction cuts. Table 5.3 summarises the PID systematic uncertainties assigned to each of the signal event classes.

\subsubsection{Hadronic Candidate and Event Topology Cuts}

Event reduction cuts placed on variables relating to the hadronic candidate or event topology are considered individually for potential systematic differences in the signal selection efficiency between MC and experimental data. Four variables are identified as potential sources of uncertainty due to non-uniform distributions in MC data of correctly reconstructed candidates. These are the minimum daughter momentum cut, the vertex $\chi^{2}$ probability cut, the decay length cut which forms part of the $K_{S}^{0}$ veto in $B \rightarrow X_{d} \gamma$ candidate reconstruction and the NN cut.

Systematic uncertainties from placing cuts on these variables are determined by taking advantage of the dominant signal event contributions in fits to both the high and low 
mass region $B \rightarrow X_{s} \gamma$ candidate experimental data. In all cases the $B \rightarrow X_{s} \gamma$ candidate distributions for these variables are assumed to be a good representation of the corresponding $B \rightarrow X_{d} \gamma$ candidate distributions. Therefore the systematic uncertainty estimated from $B \rightarrow X_{s} \gamma$ data is also directly applicable to $B \rightarrow X_{d} \gamma$ data where the signal contribution in experimental data is not significant enough to allow an independent investigation. For each variable the cut is varied locally in MC and experimental data. The PDF parameterisations and fits to experimental data are then repeated for the new cut value and the corresponding value of $n_{\text {sig }} / \epsilon_{M C}$ calculated. This is then normalised to the value of $n_{s i g} / \epsilon_{M C}$ from the default cut value and the average deviation relative to the default value is then assigned as the systematic error for that variable.

Figure 5.2 shows the variation in $n_{\text {sig }} / \epsilon_{M C}$ with respect to the default cut value for high and low mass $B \rightarrow X_{s} \gamma$ candidate data for the minimum daughter momentum cut, $\left|p_{\text {min }}\right|$. The cuts are varied by $\pm 0.1 \mathrm{GeV} / c$ in steps of $0.025 \mathrm{GeV} / c$, which corresponds to the step size used in cut optimisation. Across this range an average local deviation of $1.0 \%$ and $1.7 \%$ are observed for $B \rightarrow X_{s}^{L} \gamma$ and $B \rightarrow X_{s}^{H} \gamma$ candidate data respectively.

Figure 5.3 shows the variation in $n_{\text {sig }} / \epsilon_{M C}$ with respect to the default cut value for high and low mass $B \rightarrow X_{s} \gamma$ candidate data for the vertex $\chi^{2}$ probability cut. The cuts are varied from $0.1 \%$ to $2.5 \%$ and across this range an average local deviation of $0.5 \%$ and $1.0 \%$ are observed for $B \rightarrow X_{s}^{L} \gamma$ and $B \rightarrow X_{s}^{H} \gamma$ candidate data respectively.

Figure 5.4 shows the variation in $n_{\text {sig }} / \epsilon_{M C}$ with respect to the default cut value for high and low mass $B \rightarrow X_{s} \gamma$ candidate data for the vertex decay length cut, $x_{D E C}$. The cuts are varied by $\pm 0.1 \mathrm{~cm}$ and across this range an average local deviation of $0.2 \%$ and $0.4 \%$ are observed for $B \rightarrow X_{s}^{L} \gamma$ and $B \rightarrow X_{s}^{H} \gamma$ candidate data respectively. This systematic uncertainty only applies to $B \rightarrow X_{d} \gamma$ candidate data as the decay length cut forms part of the $K_{S}^{0}$ veto which is only applied to these data.

Figure 5.5 shows the variation in $n_{s i g} / \epsilon_{M C}$ with respect to the default cut value for high and low mass $B \rightarrow X_{s} \gamma$ candidate data for the NN cut. The cuts are nominally varied by \pm 0.1 ; however, the default cut value of 0.92 for the low mass $\mathrm{NN}$ cut restricts the upper limit of variation to 0.98 . The cuts are varied in steps of 0.02 which corresponds to the step size used for this variable during the cut optimisation. Across this range an average local deviations of $2.5 \%$ and $3.1 \%$ are observed for $B \rightarrow X_{s}^{L} \gamma$ and $B \rightarrow X_{s}^{H} \gamma$ candidate data respectively. 


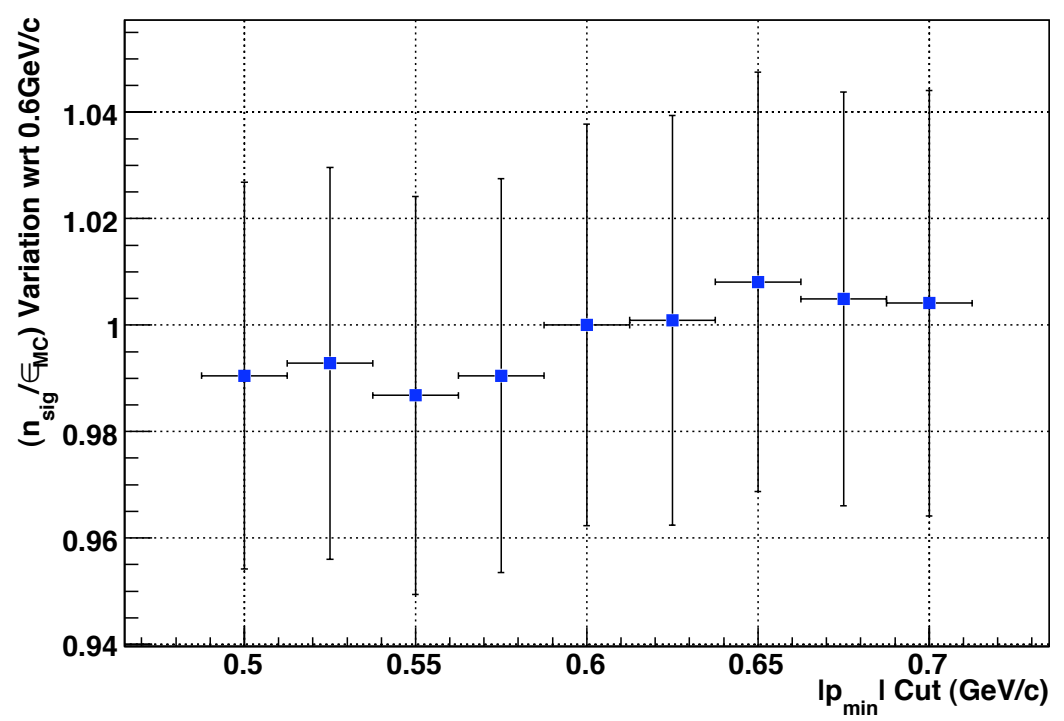

(a) $B \rightarrow X_{s}^{L} \gamma$ data, the default cut is $0.6 \mathrm{GeV} / c^{2}$. From these variations an average systematic uncertainty of $1.0 \%$ is assigned.

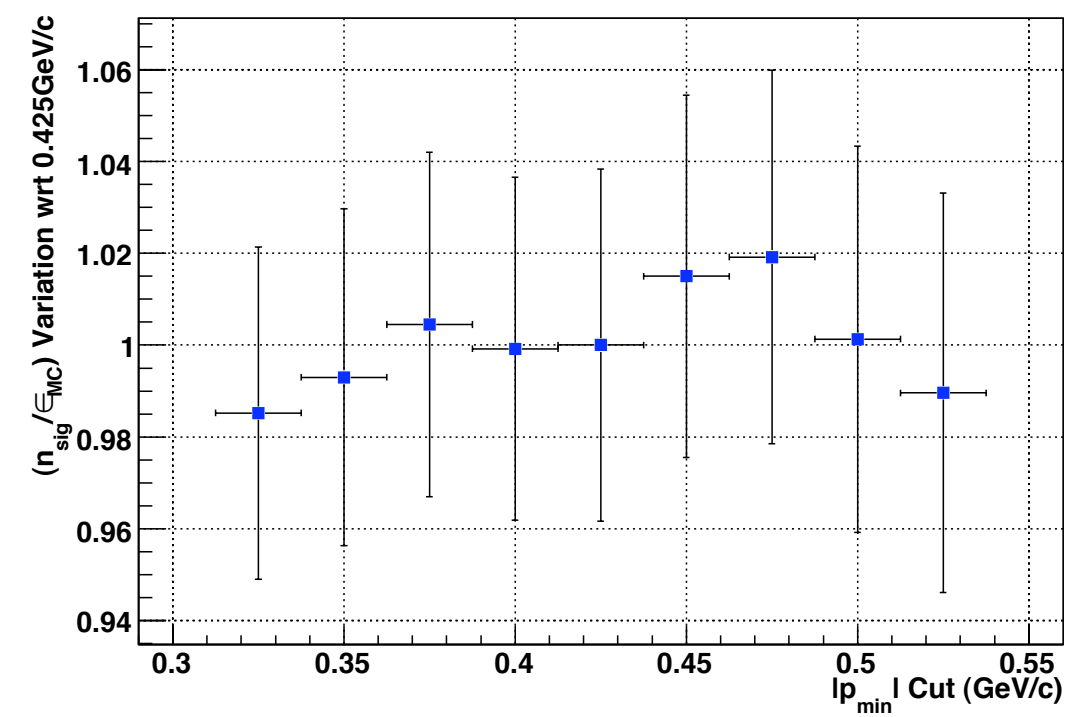

(b) $B \rightarrow X_{s}^{H} \gamma$ data, the default cut is $0.425 \mathrm{GeV} / c^{2}$. From these variations an average systematic uncertainty of $1.7 \%$ is assigned.

Figure 5.2: Systematic variation of $n_{s i g} / \epsilon_{M C}$ as a function of $\left|p_{\min }\right|$ cut, with respect to the default analysis cut for fits to (a) $B \rightarrow X_{s}^{L} \gamma$ data (b) $B \rightarrow X_{s}^{H} \gamma$ data. The vertical error bars represent the statistical uncertainty in fits to experimental data of the parameter $n_{\text {sig }}$. 


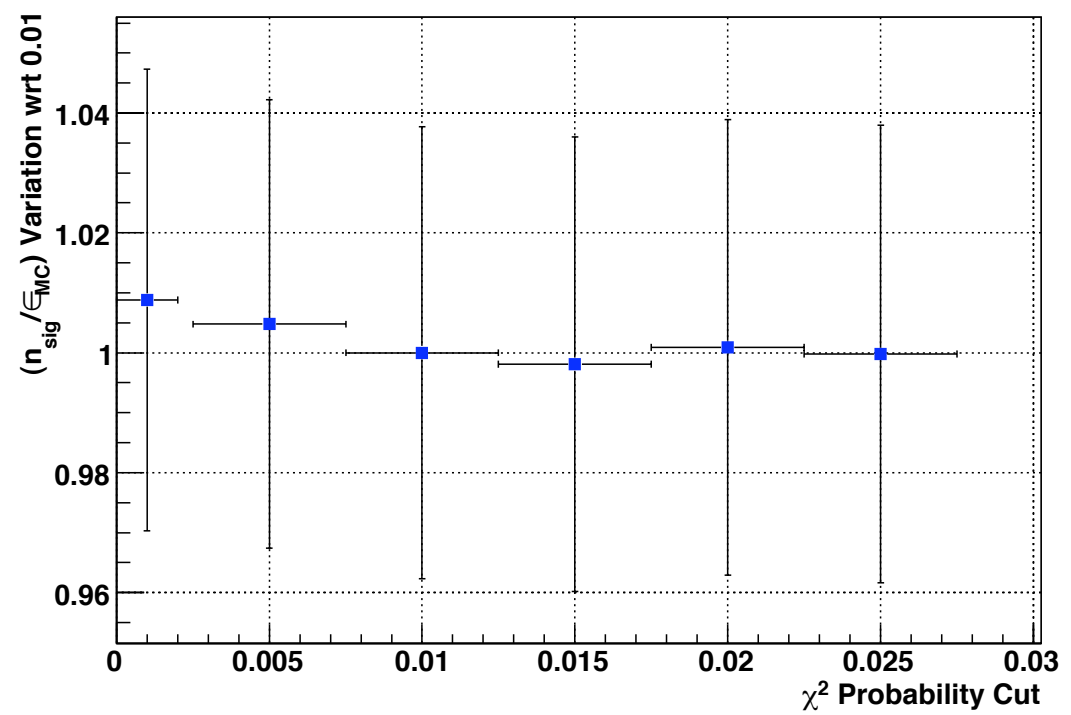

(a) $B \rightarrow X_{s}^{L} \gamma$ data, the default cut is 0.01 . From these variations an average systematic uncertainty of $0.5 \%$ is assigned.

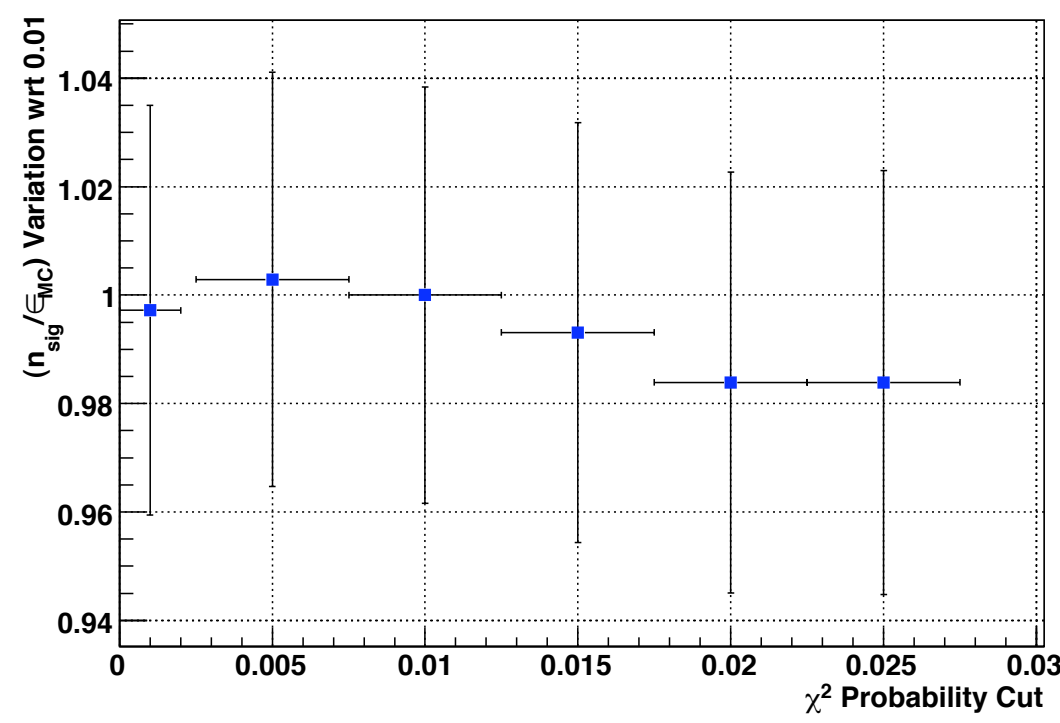

(b) $B \rightarrow X_{s}^{H} \gamma$ data, the default cut is 0.01 . From these variations an average systematic uncertainty of $1.0 \%$ is assigned.

Figure 5.3: Systematic variation of $n_{s i g} / \epsilon_{M C}$ as a function of $\chi^{2}$ probability cut, with respect to the default analysis cut for fits to (a) $B \rightarrow X_{s}^{L} \gamma$ data (b) $B \rightarrow X_{s}^{H} \gamma$ data. The vertical error bars represent the statistical uncertainty in fits to experimental data of the parameter $n_{s i g}$. 


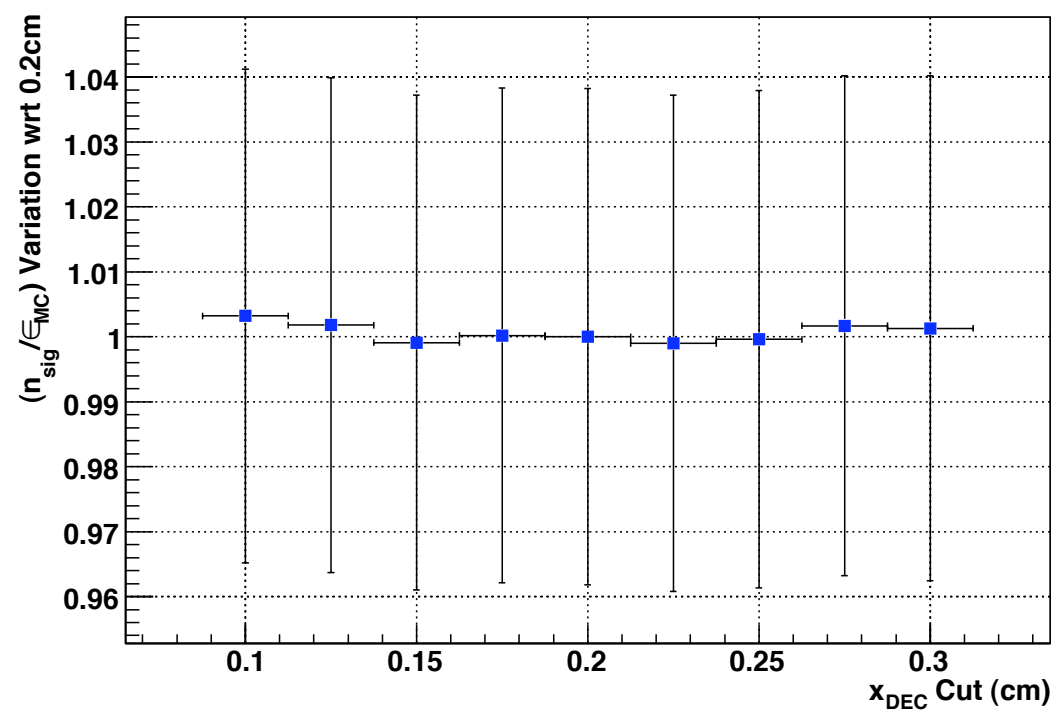

(a) $B \rightarrow X_{s}^{L} \gamma$ data, the default cut in $B \rightarrow X_{d}^{L} \gamma$ data is $0.2 \mathrm{~cm}$. From these variations an average systematic uncertainty of $0.2 \%$ is assigned.

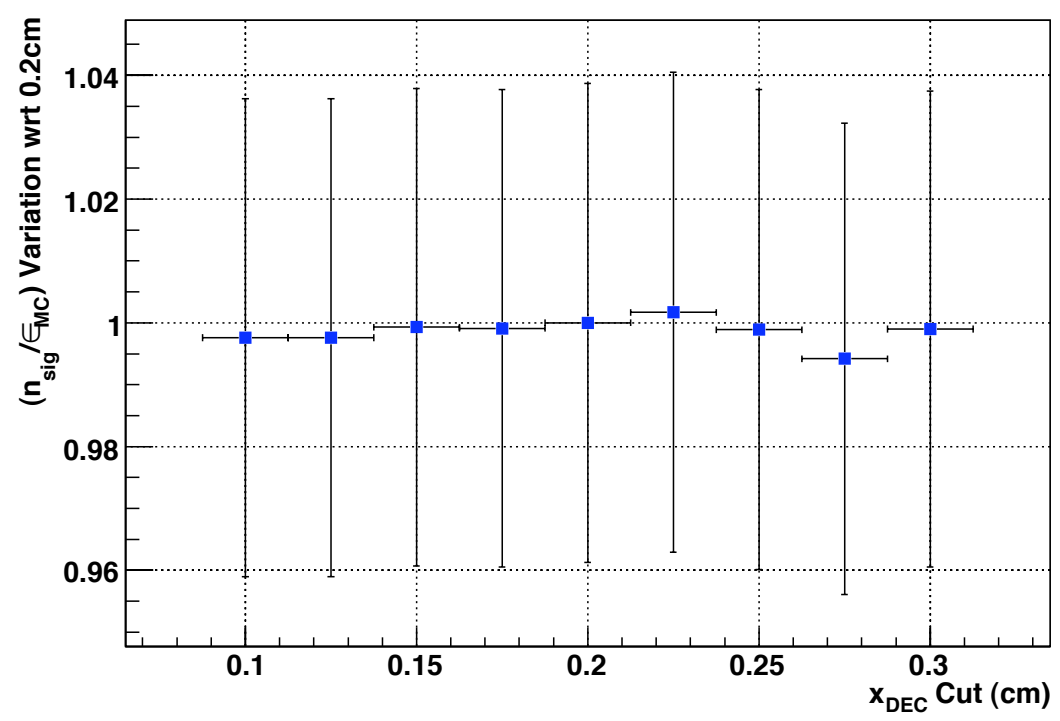

(b) $B \rightarrow X_{s}^{H} \gamma$ data, the default cut in $B \rightarrow X_{d}^{H} \gamma$ data is $0.2 \mathrm{~cm}$. From these variations an average systematic uncertainty of $0.4 \%$ is assigned.

FIgURE 5.4: Systematic variation of $n_{s i g} / \epsilon_{M C}$ as a function of $x_{D E C}$ cut, with respect to the default analysis cut for fits to (a) $B \rightarrow X_{s}^{L} \gamma$ data (b) $B \rightarrow X_{s}^{H} \gamma$ data. The vertical error bars represent the statistical uncertainty in fits to experimental data of the parameter $n_{\text {sig }}$. 


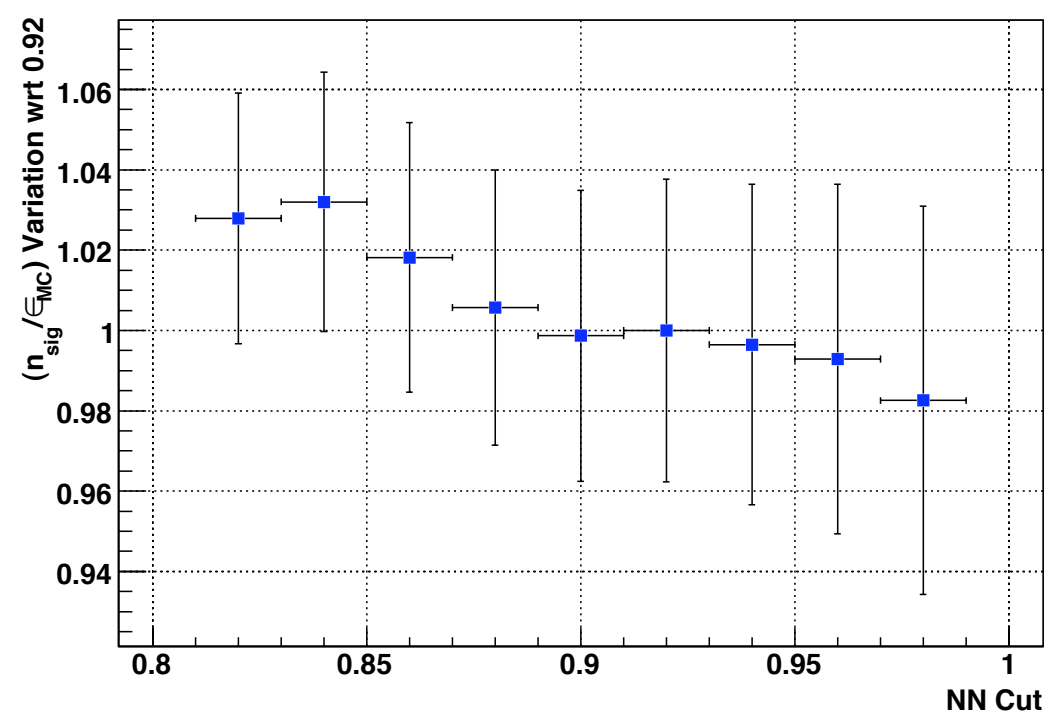

(a) $B \rightarrow X_{s}^{L} \gamma$ data, the default cut is 0.92 . From these variations an average systematic uncertainty of $2.5 \%$ is assigned.

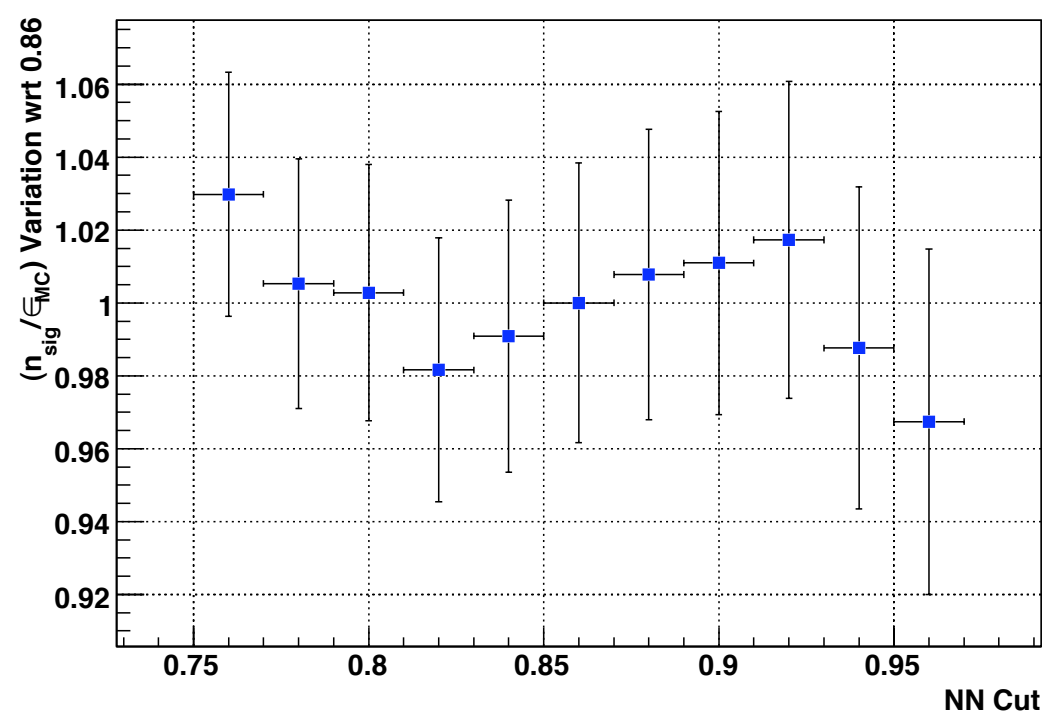

(b) $B \rightarrow X_{s}^{H} \gamma$ data, the default cut is 0.86 . From these variations an average systematic uncertainty of $3.1 \%$ is assigned.

FIgURE 5.5: Systematic variation of $n_{s i g} / \epsilon_{M C}$ as a function of NN cut, with respect to the default analysis cut for fits to (a) $B \rightarrow X_{s}^{L} \gamma$ data (b) $B \rightarrow X_{s}^{H} \gamma$ data. The vertical error bars represent the statistical uncertainty in fits to experimental data of the parameter $n_{\text {sig }}$. 


\subsubsection{Fit Uncertainties}

Event hypothesis yields and PDF parameters which are not floated in the EMLF to experimental data can lead to systematic differences in MC and experimental data. These difference can arise if the simulation does not accurately reflect the fit variable distribution shapes and normalisations or if the size of the MC data sample used to determine fixed parameters leads to significant statistical uncertainties on those estimated parameter values. Furthermore biases observed in toy MC studies indicate potential systematic uncertainties on the signal yield due to variables floated in the combined fit to data. Uncertainties must therefore be estimated for all potential sources of bias and for variables held constant in the fit, the details of which are described below.

\subsubsection{Fixed PDF Parameters}

Fixing PDF parameters assumes that the PDF distributions in MC data are a good model of the corresponding experimental data. However, the limited sample sizes of each MC event class results in an associated statistical error for each fitted parameter. These are the errors shown in tables 4.2, 4.3, 4.4 and 4.5. Consequently all fits to data are repeated for each fixed parameter, varying its value to the extremes of corresponding statistical error. The average variation in $n_{s i g}$ is then assigned as the systematic uncertainty due to that parameter. The systematic errors for all fixed variables in a given PDF are then combined, taking any correlations between parameters in the PDF fit into account. The correlation matrix between fixed PDF parameters, $\mathcal{C}$, is calculated from the fits to $\mathrm{MC}$ data discussed in section 4.4. The average systematic variations on $n_{\text {sig }}$ are expressed as an error vector $\epsilon$ whose size is equal to the number of fixed PDF parameters. This results in an overall systematic uncertainty for that PDF, $\epsilon^{\prime}$, given by,

$$
\epsilon^{\prime}=\sqrt{\boldsymbol{\epsilon}^{T} \mathcal{C} \epsilon}
$$

Systematic uncertainties for all PDFs in a given fit to data are combined in quadrature to give an overall uncertainty due to all fixed parameters in that fit.

Tables 5.4 and 5.5 show the calculated uncertainties for each fixed parameter from $m_{E S}^{\prime}$ and $\Delta E$ PDF distributions respectively, as well as the associated combined uncertainty for those variables. This gives a total systematic uncertainty of $0.3 \%$ for $B \rightarrow X_{s}^{L} \gamma$ data, $2.9 \%$ for $B \rightarrow X_{s}^{H} \gamma$ data, $5.9 \%$ for $B \rightarrow X_{d}^{L} \gamma$ data and $3.6 \%$ for $B \rightarrow X_{d}^{H} \gamma$ data due to fixed PDF parameters.

The 2D histogram PDFs which parameterise cross feed distributions do not have parameters to vary. However, each bin of the PDF does have an associated statistical error 


\begin{tabular}{|l|l|l|l|l|l|}
\hline Hypothesis & Parameter & $B \rightarrow X_{s}^{L} \gamma$ & $B \rightarrow X_{s}^{H} \gamma$ & $B \rightarrow X_{d}^{L} \gamma$ & $B \rightarrow X_{d}^{H} \gamma$ \\
\hline \hline \multirow{3}{*}{ Signal } & $\mu$ & $0.01 \%$ & $0.01 \%$ & $0.03 \%$ & $0.23 \%$ \\
& $\sigma$ & $0.02 \%$ & $0.11 \%$ & $0.10 \%$ & $0.48 \%$ \\
& $\alpha$ & $0.04 \%$ & $0.20 \%$ & $0.14 \%$ & $0.70 \%$ \\
& $n$ & $0.03 \%$ & $0.12 \%$ & $0.08 \%$ & $0.32 \%$ \\
\cline { 2 - 6 } & combined & $0.02 \%$ & $0.10 \%$ & $0.09 \%$ & $0.47 \%$ \\
\hline \hline \multirow{3}{*}{$X_{s}$ PID cross-feed } & $\mu$ & - & - & $0.12 \%$ & $0.03 \%$ \\
& $\sigma$ & - & - & $0.40 \%$ & $0.20 \%$ \\
& $\alpha$ & - & - & $0.62 \%$ & $0.28 \%$ \\
& $n$ & - & - & $0.41 \%$ & $0.16 \%$ \\
\cline { 2 - 6 } & combined & - & - & $0.36 \%$ & $0.13 \%$ \\
\hline \hline \multirow{3}{*}{ Generic $B \bar{B}$} & $\xi$ & $0.25 \%$ & $1.17 \%$ & $2.57 \%$ & $0.34 \%$ \\
& $p$ & $0.22 \%$ & $2.55 \%$ & $2.74 \%$ & $0.14 \%$ \\
& $\mu$ & $0.04 \%$ & $0.11 \%$ & $0.71 \%$ & $0.88 \%$ \\
& $\sigma$ & $0.12 \%$ & $1.03 \%$ & $1.65 \%$ & $1.81 \%$ \\
& $f$ & $0.25 \%$ & $2.91 \%$ & $3.69 \%$ & $3.60 \%$ \\
\cline { 2 - 6 } & combined & $0.14 \%$ & $0.74 \%$ & $2.24 \%$ & $3.04 \%$ \\
\hline \hline Continuum & $\xi$ & $0.68 \%$ & - & $12.49 \%$ & - \\
& $p$ & $0.75 \%$ & $2.77 \%$ & $14.26 \%$ & $0.16 \%$ \\
\hline \hline & Total Uncertainty & $0.30 \%$ & $2.87 \%$ & $5.51 \%$ & $3.08 \%$ \\
\cline { 2 - 6 } & combined & $0.26 \%$ & $2.77 \%$ & $5.02 \%$ & $0.16 \%$ \\
\hline
\end{tabular}

TABLE 5.4: Systematic uncertainties from fixing $m_{E S}^{\prime}$ PDF parameters in fits to experimental data.

so in principle each bin could be varied by the range of this error and the fit repeated. The combination in quadrature of the average variation in $n_{\text {sig }}$ from all bins would then give an overall uncertainty for the PDF. The fit framework used in this analysis does not allow such variations to be made without significant modification, therefore this uncertainty is not evaluated. The cross feed projection plots for fitted MC data in chapter 4 show that in the signal region these variations are generally small, therefore this is not expected to be a dominant systematic uncertainty. Neglecting this error is consistent with the previous version of this analysis [1].

\subsubsection{Agreement Between MC and Experimental Data}

The above systematic variations only account for uncertainties in fixed PDF parameters due to statistical limits of the MC data used for their determination. In the limit of infinite MC data points such uncertainties would be zero. An additional source of systematic uncertainty can arise if a parameter value is fundamentally different in the 


\begin{tabular}{|l|l|l|l|l|l|}
\hline Hypothesis & Parameter & $B \rightarrow X_{s}^{L} \gamma$ & $B \rightarrow X_{s}^{H} \gamma$ & $B \rightarrow X_{d}^{L} \gamma$ & $B \rightarrow X_{d}^{H} \gamma$ \\
\hline \hline \multirow{5}{*}{ Signal } & $\mu$ & $0.01 \%$ & $0.04 \%$ & $0.41 \%$ & $0.03 \%$ \\
& $\sigma_{L}$ & - & - & $1.14 \%$ & $0.83 \%$ \\
& $\sigma_{R}$ & - & - & $0.28 \%$ & $0.90 \%$ \\
& $\alpha_{L}$ & $0.03 \%$ & $0.11 \%$ & $0.19 \%$ & $0.54 \%$ \\
& $\alpha_{R}$ & $0.01 \%$ & $0.06 \%$ & $0.07 \%$ & $0.44 \%$ \\
\cline { 2 - 6 } & combined & $0.03 \%$ & $0.11 \%$ & $0.88 \%$ & $0.59 \%$ \\
\hline \hline \multirow{3}{*}{$X_{s}$ PID cross-feed } & $\mu$ & - & - & $1.55 \%$ & $0.75 \%$ \\
& $\sigma_{L}$ & - & - & $1.28 \%$ & $0.56 \%$ \\
& $\sigma_{R}$ & - & - & $0.74 \%$ & $0.42 \%$ \\
& $\alpha_{L}$ & - & - & $0.61 \%$ & $0.28 \%$ \\
& $\alpha_{R}$ & - & - & $0.02 \%$ & $0.05 \%$ \\
\cline { 2 - 6 } & combined & - & - & $0.52 \%$ & $0.24 \%$ \\
\hline \hline \multirow{5}{*}{ Generic $B \bar{B}$} & $a_{1}$ & $0.01 \%$ & $0.03 \%$ & $0.08 \%$ & $0.20 \%$ \\
& $a_{2}$ & $0.08 \%$ & $0.17 \%$ & $1.69 \%$ & $1.82 \%$ \\
\cline { 2 - 6 } & combined & $0.07 \%$ & $0.15 \%$ & $1.74 \%$ & $1.69 \%$ \\
\hline \hline Continuum & $a_{1}$ & $0.01 \%$ & - & $0.04 \%$ & - \\
\cline { 2 - 6 } & combined & $0.01 \%$ & - & $0.04 \%$ & - \\
\hline \hline & Total Uncertainty & $0.08 \%$ & $0.19 \%$ & $2.02 \%$ & $1.81 \%$ \\
\hline
\end{tabular}

TABLE 5.5: Systematic uncertainties from fixing $\Delta E$ PDF parameters in fits to experimental data.

MC data and experimental data due to modelling errors. However, such an uncertainty will only be significant if the difference is much greater than the statistical variations already considered.

Signal PDF distributions use the $B \rightarrow X_{s} \gamma$ fits to data as a control sample to compare $\mathrm{MC}$ and experimental data distributions. In these data the contribution of signal events dominates. Parameters which are known to not be modelled well in the MC data, specifically the widths of $\Delta E$ distributions, are measured and used to correct the signal shapes in $B \rightarrow X_{d} \gamma$ fits to data. The remaining fixed PDF parameters for these distributions show no significant difference between MC and experimental data beyond the statistical uncertainty of those parameters in the control sample.

Cross-feed distributions modelled as 2D histograms do not depend on fixed PDF parameters; however, the shape of these histograms could be expected to show systematic differences between MC and experimental data particularly for decays generated from JETSET. The application of weights to the $B \rightarrow X_{s} \gamma$ MC data discussed in section 4.2 can indicate the potential for such differences. In all cases the difference in shape between weighted and unweighted bins for high mass $B \rightarrow X_{s} \gamma \mathrm{MC}$ data histograms 
were found to be negligible. The only significant differences observed were in the overall normalisations which are discussed below.

Variations of fixed PDF parameters for generic $B$ background PDFs contribute significantly to the overall fit uncertainty. Tables 5.4 and 5.5 show that this can be attributed to the peaking component in $m_{E S}^{\prime}$ as generally the Argus function parameter variations in $m_{E S}^{\prime}$ for this PDF are anti-correlated and cancel. The peaking backgrounds typically from events with low multiplicity $B$ decays which have a similar final state to the signal mode reconstructed. The previous version of this analysis [1] carried out dedicated studies using charmless $B \rightarrow X \pi^{0}$ and $B \rightarrow X \eta$ MC samples to model such backgrounds with independent PDF distributions. No significant difference was observed between parameters of the peaking components from these distributions with those peaking backgrounds in other generic $B$ decays. Therefore in this analysis they were modelled with a combined PDF distribution determined from the generic sample without such events vetoed. Despite a larger generated generic sample in this analysis, the optimised cuts are generally tighter than before leading to similar numbers of events used to fit the PDFs for this background. The resulting statistical variations on fixed parameters are hence comparable. The previous analysis determined that these variations were in excess of any underlying systematic differences between the MC and experimental distribution parameters and this assumption is therefore made here too. Indeed from the statistical variations only two parameters from the $m_{E S}^{\prime}$ distributions are of specific concern due to the sensitivity of $n_{\text {sig }}$ to their respective values: the Gaussian width and relative fraction of Argus normalisation to Gaussian normalisation. The fraction parameter uncertainty is to an extent addressed in the normalisation variation discussed below. This is because the change in Gaussian normalisation will generally be absorbed by the similarly peaking signal distribution, hence biasing $n_{\text {sig }}$, whereas the change in Argus normalisation will generally be absorbed by the floated continuum Argus. Indeed variations in the Generic $B$ normalisations are anti-correlated with $n_{\text {sig }}$. Knowledge of potential uncertainties from the Gaussian width can be determined from signal distributions. The signal width in $m_{E S}^{\prime}$ is well modelled by the MC data as shown from the fits to $B \rightarrow X_{s} \gamma$ experimental data. This width represents the detector resolution limit of this variable for correctly reconstructed candidates. The possibility of a peaking background having width narrower than this limit is physically unreasonable. With the exception of high mass $B \rightarrow X_{s} \gamma$ candidates the negative statistical variation of the fixed peaking background width takes it below the signal width as can be seen from tables 4.2 and 4.4. Hence the variations made are already likely to be too conservative; in fact the variations in $n_{\text {sig }}$ from varying the the Gaussian widths are generally asymmetric with the negative, non-physical extreme giving the larger uncertainty. This is because a wider peaking component leads to this background having a 


\begin{tabular}{|l|l|l|l|l|}
\hline Yield & $B \rightarrow X_{s}^{L} \gamma$ & $B \rightarrow X_{s}^{H} \gamma$ & $B \rightarrow X_{d}^{L} \gamma$ & $B \rightarrow X_{d}^{H} \gamma$ \\
\hline$n_{s c f}$ & $0.96 \%$ & $3.39 \%$ & $1.12 \%$ & $1.92 \%$ \\
$n_{m c f}$ & $1.99 \%$ & $0.86 \%$ & $0.79 \%$ & $0.52 \%$ \\
$n_{P I D}^{X s}$ & - & - & $3.11 \%$ & $1.04 \%$ \\
$n_{s s}^{X s}$ & - & - & $1.61 \%$ & $3.21 \%$ \\
$n_{m c f}^{X s}$ & - & - & $2.97 \%$ & $0.24 \%$ \\
$n_{\text {GenB }}$ & $0.61 \%$ & $2.78 \%$ & $7.33 \%$ & $8.23 \%$ \\
\hline Total Uncertainty & $2.29 \%$ & $4.47 \%$ & $8.76 \%$ & $9.12 \%$ \\
\hline
\end{tabular}

TABLE 5.6: Systematic uncertainties from fixing event hypothesis yields in fits to experimental data.

more continuum-like shape, thus biasing the signal yield less.

The continuum PDF fixed parameter variations are only a significant concern in the low mass $B \rightarrow X_{d} \gamma$ fit to experimental data where it was not possible to float the Argus slope parameter due to toy study fits failing when this freedom was allowed in the combined yield fit. However, the statistical variations made are relatively large due to low MC data sample sizes after event selection cuts. The statistical variations are therefore assumed to be sufficient to parameterise potential systematic uncertainties.

\subsubsection{Fixed Event Class Normalisations}

In fits to experimental data the relative normalisations of cross feed and generic $B$ PDF contributions are fixed, thereby assuming the MC data accurately represent the relative reconstruction efficiencies of candidates within these event classes. However, not all of the contributions are necessarily well modelled, hence uncertainties exist in these normalisations. To estimate the associated systematic uncertainty the fits to data are repeated assuming a predetermined 'conservative' variation in each normalisation and taking the average variation in $n_{\text {sig }}$ as the uncertainty for that event class. Individual uncertainties from each event class are combined in quadrature to give an overall systematic uncertainty for a particular data fit.

Table 5.6 summarises the uncertainties estimated from variations made to each event class normalisation in fits to experimental data. With the exception of PID cross feed, cross feed contributions are varied by $\pm 30 \%$. This is because the application of weights to $B \rightarrow X_{s} \gamma \mathrm{MC}$ data discussed in section 4.2 do not vary the relative normalisations by more than this amount. This is therefore used as an estimate of how much normalisations could vary for all cross feed distributions. The iterative updating of these yields in fits to data means that this is likely to be conservative variation as in those iterations all cross-feed normalisations converged. The PID cross feed only contributes to $B \rightarrow X_{d} \gamma$ 


\begin{tabular}{|l|l|l|l|l|}
\hline Parameter & $B \rightarrow X_{s}^{L} \gamma$ & $B \rightarrow X_{s}^{H} \gamma$ & $B \rightarrow X_{d}^{L} \gamma$ & $B \rightarrow X_{d}^{H} \gamma$ \\
\hline$n_{\text {sig }}$ & $0.5 \%$ & $0.1 \%$ & $2.9 \%$ & $1.0 \%$ \\
$n_{\text {cont }}$ & $0.0 \%$ & $0.1 \%$ & $0.1 \%$ & $0.2 \%$ \\
$\sigma_{L}$ & $0.2 \%$ & $0.8 \%$ & - & - \\
$\sigma_{R}$ & $0.0 \%$ & $0.2 \%$ & - & - \\
$\xi$ & - & $0.0 \%$ & - & $0.7 \%$ \\
$a_{1}$ & - & $0.0 \%$ & - & $0.0 \%$ \\
\hline Combined Uncertainty & $0.6 \%$ & $0.9 \%$ & $2.9 \%$ & $1.3 \%$ \\
\hline
\end{tabular}

TABLE 5.7: Systematic uncertainties from bias on parameters floated in fits to experimental data.

data samples and is formed of correctly reconstructed modes which have been measured in the corresponding $B \rightarrow X_{s} \gamma$ data to a statistical accuracy of lower than $5 \%$ in both the high and low mass regions. Control sample studies by the PID group show that the rate of charged kaons being misidentified as pions is less than $10 \%$ for all momenta with reasonable agreement between $\mathrm{MC}$ and experimental data. For these reasons the PID cross feed normalisation is varied by $\pm 10 \%$ or \pm 1 event, whichever is greater.

Studies of generic $B$ MC data find the generated events of such candidates are generally not dominated by a particular $B$ decay. As discussed above, this background was parameterised differently in the previous version of this analysis. The normalisation is varied by $\pm 50 \%$. This is to conservatively allow for the possibility of a significantly larger or smaller peaking $m_{E S}^{\prime}$ background component which, as seen from the statistical variations above, is potentially a very significant systematic uncertainty. It should be noted that it is potentially possible to make a more accurate estimate of the agreement of generic $B$ background normalisation between $\mathrm{MC}$ and experimental data. The hadronic mass distribution of $B \rightarrow X_{s}^{H} \gamma$ candidates in generic $B$ events contains a peak at the $D^{0}$ where the $D^{0}$ decay is identical to the an $X_{s}$ final state, has been correctly reconstructed but combined with a photon from elsewhere in the event. The corresponding peak is also observed in the hadronic mass distribution of the experimental data. A detailed long term study may therefore be possible using this peak to better understand any difference between MC and experimental data normalisation.

\subsubsection{Fit Bias}

Bias on the signal yield from parameters floated in fits to data are a potential source of systematic uncertainty. The embedded toy studies pulls calculated in section 4.5.2 are used to correct floated parameters for biases in their own value in fits to experimental data. To evaluate the effect of such biases on signal yields each fit is repeated fixing a 
given parameter to its bias corrected value and allowing all other parameters to float as normal. The resulting variation in the signal yield is taken as the uncertainty due to the bias from that parameter. The uncertainty on signal yield itself is simply the relative size of its own bias correction. Table 5.7 lists the uncertainties calculated from this method for each fit to data. The combined uncertainty takes correlations between the floated variables into account by using correlation matrices calculated from the default fits to experimental data and applying (5.2).

\subsubsection{Signal Model Uncertainties}

Assumptions made in modelling signal decay distributions in MC data can have associated systematic uncertainties if the model is not an accurate representation of true signal events or has any associated uncertainty itself. This section considers potential sources of error on the signal efficiency calculated from signal MC samples and quantifies the corresponding systematic uncertainties.

\subsubsection{Photon Spectrum Model}

Systematic variations to the KN photon spectrum parameterisation, given numerically in chapter 1 , are used to estimate the associated uncertainty of the signal efficiency due to this model. Signal MC data with a total 2,122,000 events are generated for both $B \rightarrow X_{s} \gamma$ and $B \rightarrow X_{d} \gamma$ decays under each set of alternative KN parameters. From these data the relative contribution of signal states in the range $1.0 \leq m_{X} \leq 2.0 \mathrm{GeV} / c^{2}$ before cuts are determined. To estimate the number of events passing all cuts under an alternative model the analysis is not repeated on these new data. Instead all generated MC events in the signal mass range, $N_{\text {sig }}$, are separated into 50 equal width bins of generated hadronic mass, each containing $n_{i}(i=1 . .50)$ events. These are then normalised to the total number of events in the signal mass range, giving a proportion of events in each bin, $\zeta_{i}=n_{i} / N_{s i g}$. The proportion of events in that bin are then compared to the corresponding proportion under the default $\mathrm{KN}$ parameterisation, $\zeta_{i}^{\prime}$, through the relation $\zeta_{i} / \zeta_{i}^{\prime}$. This ratio of proportions is interpreted as a weight which, when applied to the default KN model, maps the default parameterisation onto the new parameterisation. Thus a weighted sum based on the generated hadronic mass of correctly reconstructed events passing all cuts in the default model gives the expected number of events which will pass all cuts under that alternative parameterisation.

Figure 5.6 shows plots of $\zeta_{i} / \zeta_{i}^{\prime}$ across the mass range for each alternative KN model for both $B \rightarrow X_{s} \gamma$ and $B \rightarrow X_{d} \gamma$ transitions. To simplify the calculation of weighted event sums, the weights are parameterised as a linear function in hadronic mass by 

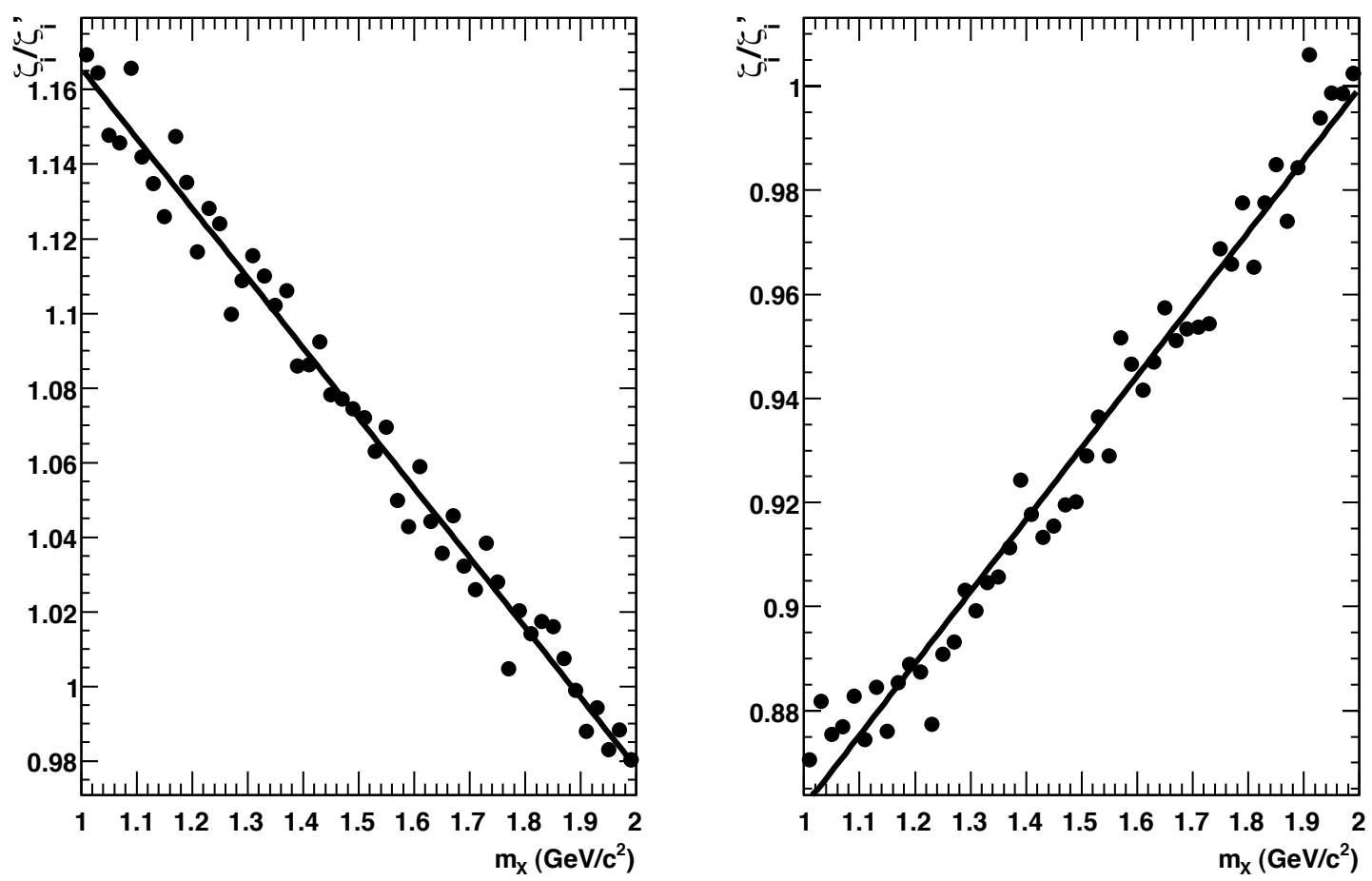

(a) $B \rightarrow X_{s} \gamma$ alternative $\mathrm{KN}$ mass weights and fitted linear polynomials. The left plot shows the weight distribution needed when using the default parameterisation, $\left(m_{b}, \mu_{\pi}^{2}\right)=\left(4.65 \mathrm{GeV} / c^{2}, 0.52 \mathrm{GeV}^{2}\right)$, to emulate the parameterisation $\left(4.70 \mathrm{GeV} / c^{2}, 0.45 \mathrm{GeV}^{2}\right)$ and the right plot the corresponding distribution to emulate the parameterisation $\left(4.60 \mathrm{GeV} / c^{2}, 0.60 \mathrm{GeV}^{2}\right)$.
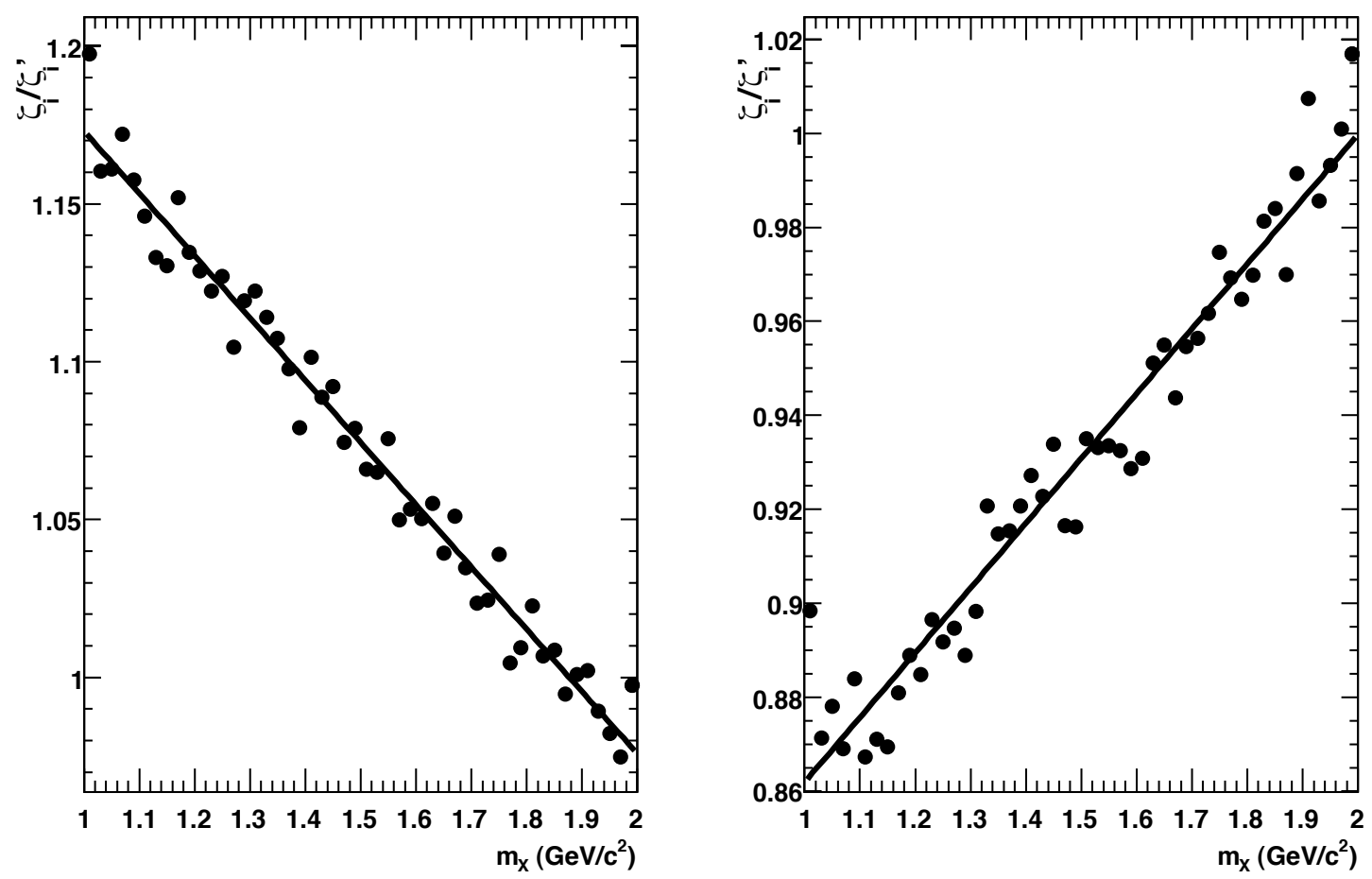

(b) $B \rightarrow X_{d} \gamma$ alternative $\mathrm{KN}$ mass weights and fitted linear polynomials. The left plot shows the weight distribution needed when using the default parameterisation, $\left(m_{b}, \mu_{\pi}^{2}\right)=\left(4.65 \mathrm{GeV} / c^{2}, 0.52 \mathrm{GeV}^{2}\right)$, to emulate the parameterisation $\left(4.70 \mathrm{GeV} / c^{2}, 0.45 \mathrm{GeV}^{2}\right)$ and the right plot the corresponding distribution to emulate the parameterisation $\left(4.60 \mathrm{GeV} / c^{2}, 0.60 \mathrm{GeV}^{2}\right)$.

Figure 5.6: Calculated hadronic mass event weight distributions for emulation of alternative KN model parameterisations from the default in (a) $B \rightarrow X_{s} \gamma$ MC data and (b) $B \rightarrow X_{d} \gamma \mathrm{MC}$ data. Each distribution is fitted with a linear polynomial function. 


\begin{tabular}{|l|l|l|}
\hline KN parameterisation & Weight Function & $\epsilon_{M C}$ \\
\hline$\left(4.65 \mathrm{GeV} / c^{2}, 0.52 \mathrm{GeV}^{2}\right)$ & 1.000 & $1.71 \%$ \\
$\left(4.60 \mathrm{GeV} / c^{2}, 0.60 \mathrm{GeV}^{2}\right)$ & $0.733+0.148 m_{X}$ & $1.82 \%$ \\
$\left(4.70 \mathrm{GeV} / c^{2}, 0.45 \mathrm{GeV}^{2}\right)$ & $1.272-0.176 m_{X}$ & $1.62 \%$ \\
\hline
\end{tabular}

TABLE 5.8: Fitted weight functions and calculated MC data efficiency for alternative $\mathrm{KN}$ parameterisations in $B \rightarrow X_{s} \gamma \mathrm{MC}$ data.

\begin{tabular}{|l|l|l|}
\hline KN parameterisation & Weight Function & $\epsilon_{M C}$ \\
\hline$\left(4.65 \mathrm{GeV} / c^{2}, 0.52 \mathrm{GeV}^{2}\right)$ & 1.000 & $1.74 \%$ \\
$\left(4.60 \mathrm{GeV} / c^{2}, 0.60 \mathrm{GeV}^{2}\right)$ & $0.733+0.147 m_{X}$ & $1.85 \%$ \\
$\left(4.70 \mathrm{GeV} / c^{2}, 0.45 \mathrm{GeV}^{2}\right)$ & $1.285-0.185 m_{X}$ & $1.64 \%$ \\
\hline
\end{tabular}

TABLE 5.9: Fitted weight functions and calculated MC data efficiency for alternative KN parameterisations in $B \rightarrow X_{d} \gamma \mathrm{MC}$ data.

fitting 1D polynomials to the corresponding histograms. Tables 5.8 and 5.9 list the respective event weight functions and calculated $\mathrm{MC}$ data efficiencies for different $\mathrm{KN}$ models in $B \rightarrow X_{s} \gamma$ and $B \rightarrow X_{d} \gamma$ MC data. From these efficiency variations an average uncertainty of $5.8 \%$ and $6.0 \%$ are assigned to the measured $B \rightarrow X_{s}^{H} \gamma$ and $B \rightarrow X_{d}^{H} \gamma$ BFs respectively.

\subsubsection{JETSET Phase Space Model}

Uncertainties in the signal efficiency due to differences between the relative distribution of final states for the JETSET decay model in KN signal MC data and their true distribution in experimental data are evaluated for $B \rightarrow X_{s}^{H} \gamma$ and $B \rightarrow X_{d}^{H} \gamma$ decays.

As discussed in section 4.2, correction weights have been applied to $B \rightarrow X_{s}^{H} \gamma \mathrm{MC}$ data before calculating the signal efficiency. Therefore the only remaining uncertainty in the distribution of these decays in experimental data arises from the statistical uncertainty of these measured weights. The weights and their associated uncertainties are given in table B.6 of appendix B. For each signal mode the applied weight is varied to the extremes of its quoted error while keeping the other signal weights constant. The total signal efficiency, $\epsilon_{M C}$, is then recalculated and the average relative variation in the efficiency assigned as the associated uncertainty for that mode. The uncertainties for each mode are then added in quadrature to give an overall systematic uncertainty due to the relative distribution of signal states in $B \rightarrow X_{s}^{H} \gamma$ data. Table 5.10 list the relative uncertainty of each signal mode due to its weight. The combined uncertainty for all signal modes is $3.5 \%$. 


\begin{tabular}{|l|l|}
\hline Signal Mode & Uncertainty \\
\hline$B^{0} \rightarrow K^{+} \pi^{-} \gamma$ & $1.0 \%$ \\
$B^{0} \rightarrow K^{+} \pi^{0} \gamma$ & $0.5 \%$ \\
$B^{0} \rightarrow K^{+} \pi^{-} \pi^{+} \gamma$ & $0.6 \%$ \\
$B^{0} \rightarrow K^{+} \pi^{-} \pi^{0} \gamma$ & $1.2 \%$ \\
$B^{0} \rightarrow K^{+} \pi^{-} \pi^{+} \pi^{-} \gamma$ & $0.9 \%$ \\
$B^{0} \rightarrow K^{+} \pi^{-} \pi^{+} \pi^{0} \gamma$ & $2.7 \%$ \\
$B^{0} \rightarrow K^{+} \eta \gamma$ & $0.7 \%$ \\
\hline All Signal & $3.5 \%$ \\
\hline
\end{tabular}

TABLE 5.10: Systematic uncertainties due to uncertainties in the weights applied to $B \rightarrow X_{s}^{H} \gamma$ signal MC data modes.

Calculating the corresponding uncertainty for $B \rightarrow X_{d}^{H} \gamma$ data is less straightforward as no information from experimental data exist and the default distribution of final states from JETSET decays is assumed to be correct when calculating $\epsilon_{M C}$. Instead an alternative model of $B \rightarrow X_{d}^{H} \gamma$ final state distributions is proposed using the known weights from $B \rightarrow X_{s}^{H} \gamma$ data, applying them to the $B \rightarrow X_{d}^{H} \gamma$ data and then taking the resulting change in $\mathrm{MC}$ efficiency as an estimate of the size of systematic variation which could occur due to the difference in final state distributions in MC and experimental data.

The alternative model of signal mode contributions for $B \rightarrow X_{d}^{H} \gamma$ data is designed to reflect the relative contribution of different final states previously measured in $B \rightarrow$ $X_{s}^{H} \gamma$ data. For these data the $s$ quark produced in the radiative penguin transition is subsequently found in either a charged or neutral $K$ meson. In substituting the $s$ quark for a $d$ quark the confined quark state substitutions listed in table 5.11 are assumed. From isospin symmetry, the $|\bar{d}\rangle$ state contributes to the $\pi^{0}, \eta$ and $\eta^{\prime}$ states in the pseudoscalar meson nonet. Under this scheme the meson states are given as [5],

$$
\pi^{0}=\frac{(|u \bar{u}\rangle-|d \bar{d}\rangle)}{\sqrt{2}}, \quad \eta=\frac{(|u \bar{u}\rangle+|d \bar{d}\rangle-2|s \bar{s}\rangle)}{\sqrt{6}}, \quad \eta^{\prime}=\frac{(|u \bar{u}\rangle+|d \bar{d}\rangle+|s \bar{s}\rangle)}{\sqrt{3}} .
$$

It follows that, ignoring factors such as mass difference which break $\mathrm{SU}(3)$ isospin symmetry, the $|\bar{d}\rangle$ state will be found in the ratio $\left(\pi^{0}: \eta: \eta^{\prime}\right)=(3: 1: 2)$ and thus the neutral kaon states can be substituted by these mesons in this ratio.

For each signal mode in $B \rightarrow X_{d}^{H} \gamma \mathrm{MC}$ data a weight can now be calculated from the known $B \rightarrow X_{s}^{H} \gamma$ weights. In generated $B \rightarrow X_{s}^{H} \gamma$ signal MC data after weights are applied, a given mode $i$ will contribute a fraction $f_{i}^{s}$ to the total number of events with hadronic mass greater than $1.0 \mathrm{GeV} / c^{2}$. Adding the $f_{i}^{s}$ of all $B \rightarrow X_{s}^{H} \gamma$ modes which, on substitution of the kaon, correspond to the signal $B \rightarrow X_{d}^{H} \gamma$ mode $j$ under 


\begin{tabular}{|l|l|l|}
\hline$s$ State & $d$ State & Meson Substitution \\
\hline$|s \bar{u}\rangle$ & $|d \bar{u}\rangle$ & $K^{-} \rightarrow \pi^{-}$ \\
$|s \bar{d}\rangle$ & $|d \bar{d}\rangle$ & $\bar{K}^{0} \rightarrow \pi^{0}, \eta, \eta^{\prime}$ \\
\hline
\end{tabular}

TABLE 5.11: Comparison of final state particles when substituting the $s$ quark for a $d$ quark.

consideration gives the expected fraction of mode $j$ events with hadronic mass greater than $1.0 \mathrm{GeV} / c^{2}, f_{j}^{d}$. The weight, $w_{j}$, to apply to $B \rightarrow X_{d}^{H} \gamma$ events of mode $j$ is then the ratio of this fraction with the original fraction of such events, $f_{j}^{d^{\prime}}$, generated in the MC data:

$$
w_{j}=\frac{f_{j}^{d}}{f_{j}^{d^{\prime}}}=\frac{\sum_{i} f_{i}^{s}}{f_{j}^{d^{\prime}}} .
$$

Table 5.12 lists all of the $B \rightarrow X_{s}^{H} \gamma$ modes corresponding to a $B \rightarrow X_{d}^{H} \gamma$ signal mode from kaon substitution as well as showing the calculated weights. The mode $B^{+} \rightarrow$ $\pi^{+} \pi^{-} \pi^{+} \pi^{0} \gamma$ can contain contributions from intermediate $\eta \rightarrow \pi^{+} \pi^{-} \pi^{0}$ transitions hence under substitution the decay $B^{+} \rightarrow K^{0} \pi^{+} \gamma$ also contributes partially. This is accounted for in the calculation of the weight for this mode using the world average rate of $\eta \rightarrow$ $\pi^{+} \pi^{-} \pi^{0}$ decays as a proportion of all potential $\eta$ meson decays [14].

After weights are applied to the $B \rightarrow X_{d}^{H} \gamma \mathrm{MC}$ data the signal efficiency, $\epsilon_{M C}$, is calculated to be $1.54 \%$ which is a variation of $12.0 \%$ relative to the default efficiency used to calculate the measured BF. This is therefore assigned as the estimate of systematic uncertainty due to potential differences in $\mathrm{MC}$ and experimental data final state distributions for $B \rightarrow X_{d}^{H} \gamma$ data.

\subsubsection{Resonant Final States}

Uncertainties in the calculated signal efficiency for measurements in the low mass region exist due to the assumed relative contributions of different resonant states. In calculating $\epsilon_{M C}$ the relative resonant contributions are assumed to reflect the best measured values taken from [14]. Each measurement has a quoted error which results in a corresponding uncertainty due to that resonance in the efficiency calculation. To numerically evaluate this uncertainty the efficiency is recalculated varying the relative contribution of each resonance within the extremes of its error while leaving the other resonant contributions unchanged. The average relative variation in the efficiency is then interpreted as the uncertainty due to that resonance. Each resonance final state is assumed to have been measured independently thus the individual uncertainties are combined in quadrature to give an overall uncertainty due to all contributing resonances. Tables 5.13 lists the measured rates and associated uncertainties in the calculated signal efficiency of 


\begin{tabular}{|l|l|l|}
\hline$B \rightarrow X_{d}^{H} \gamma$ Mode & Contributing $B \rightarrow X_{s}^{H} \gamma$ Modes & Calculated Weight \\
\hline \hline$B^{0} \rightarrow \pi^{+} \pi^{-} \gamma$ & $B^{0} \rightarrow K^{+} \pi^{-} \gamma$ & 0.73 \\
\hline$B^{+} \rightarrow \pi^{+} \pi^{0} \gamma$ & $B^{0} \rightarrow K^{+} \pi^{0} \gamma$ & \\
& $B^{+} \rightarrow K^{0} \pi^{+} \gamma$ & 0.47 \\
\hline$B^{+} \rightarrow \pi^{+} \pi^{-} \pi^{+} \gamma$ & $B^{+} \rightarrow K^{+} \pi^{-} \pi^{+} \gamma$ & 1.78 \\
\hline$B^{0} \rightarrow \pi^{+} \pi^{-} \pi^{0} \gamma$ & $B^{0} \rightarrow K^{+} \pi^{-} \pi^{0} \gamma$ & \\
& $B^{0} \rightarrow K^{0} \pi^{+} \pi^{-} \gamma$ & 1.38 \\
\hline$B^{0} \rightarrow \pi^{+} \pi^{-} \pi^{+} \pi^{-} \gamma$ & $B^{0} \rightarrow K^{+} \pi^{-} \pi^{+} \pi^{-} \gamma$ & 0.77 \\
\hline$B^{+} \rightarrow \pi^{+} \pi^{-} \pi^{+} \pi^{0} \gamma$ & $B^{0} \rightarrow K^{+} \pi^{-} \pi^{+} \pi^{0} \gamma$ & \\
& $B^{+} \rightarrow K^{0} \pi^{+} \pi^{-} \pi^{+} \gamma$ & \\
& $B^{+} \rightarrow K^{0} \pi^{+} \gamma$ & 0.96 \\
\hline$B^{+} \rightarrow \pi^{+} \eta \gamma$ & $B^{+} \rightarrow K^{+} \eta \gamma$ & \\
& $B^{+} \rightarrow K^{0} \pi^{+} \gamma$ & 1.09 \\
\hline
\end{tabular}

TABLE 5.12: Weights assigned to $B \rightarrow X_{d}^{H} \gamma$ signal modes to obtain an alternative model of final state contributions. The $B \rightarrow X_{s}^{H} \gamma$ modes used to calculate each weight, as described in the text, are also shown.

\begin{tabular}{|l|l|l|}
\hline Resonant Decay & BF from $[14]$ & Uncertainty \\
\hline$B \rightarrow K^{*+} \gamma$ & $(4.0 \pm 0.3) \times 10^{-5}$ & $0.4 \%$ \\
$B \rightarrow K^{* 0} \gamma$ & $(4.0 \pm 0.2) \times 10^{-5}$ & $0.2 \%$ \\
$B \rightarrow \rho^{+} \gamma$ & $\left(8.8_{-2.5}^{+2.9}\right) \times 10^{-7}$ & $1.5 \%$ \\
$B \rightarrow \rho^{0} \gamma$ & $(9.3 \pm 2.1) \times 10^{-7}$ & $3.1 \%$ \\
$B \rightarrow \omega \gamma$ & $\left(4.6_{-1.7}^{+2.0}\right) \times 10^{-7}$ & $7.4 \%$ \\
\hline
\end{tabular}

TABLE 5.13: Uncertainty in signal efficiency due to assumed relative contribution of individual resonant final states.

resonances contributing to $B \rightarrow X_{s}^{L} \gamma$ and $B \rightarrow X_{d}^{L} \gamma$ data. The relative contribution of resonant decays to non-reconstructed final states and the proportion of events with hadronic mass less than $1.0 \mathrm{GeV} / c^{2}$ are assumed to be accurately modelled by MC data. The combined uncertainty in the signal efficiency is $0.4 \%$ for $B \rightarrow X_{s}^{L} \gamma$ data and $8.2 \%$ for the $B \rightarrow X_{d}^{L} \gamma$ data.

\subsubsection{Summary of Experimental Systematic Uncertainties}

Table 5.14 summarises all systematic uncertainty estimates detailed above. These are combined in quadrature to give an overall systematic uncertainty for each of the five sources of systematic error outlined and hence a total systematic uncertainty for each measured BF. Table 5.15 summarises the four measured BFs showing the statistical uncertainty and applying the corresponding systematic uncertainties given in table 5.14. 
The $B \rightarrow X_{s}^{L} \gamma$ systematic uncertainty is dominated by the event reductions cuts, in particular the photon selection, PID requirements and NN cut. There is no dominant systematic uncertainty for $B \rightarrow X_{s}^{H} \gamma$ decays; the event selection, fit and signal model uncertainties all have similar contributions. For both high and low mass $B \rightarrow X_{d} \gamma$ the fit uncertainties, and signal model uncertainties, become more dominant. However, both of these measurements are still statistically limited.

\subsection{Measured BF Extrapolation and Extraction of $\left|V_{t d} / V_{t s}\right|$}

Extraction of $\left|V_{t d} / V_{t s}\right|$ requires extrapolation of the measured BFs to the full $B \rightarrow$ $X_{(s, d)} \gamma$ branching ratios required to calculate $R$ in (1.67). This extrapolation is based on the MC data and hence further systematic uncertainties due to potential differences compared to the experimental data must be evaluated. This section describes each $\mathrm{BF}$ extrapolation, estimates the associated systematic uncertainties and calculates $R$ to extract $\left|V_{t d} / V_{t s}\right|$.

\subsubsection{Low Mass Region BF Extrapolation}

Measured BFs with hadronic mass less than $1.0 \mathrm{GeV} / c^{2}$ are modelled entirely as exclusive resonant transitions. Corrections for unreconstructed final states of the subsequent vector meson decays are therefore required.

For $B \rightarrow X_{s}^{L} \gamma$ data the contribution in MC data from unreconstructed final states are assumed to be accurately represented by the MC data which considers strong decay contributions of $K^{*}$ mesons as well as $K^{*} \rightarrow K \gamma$ final states. Tables B.2 and B.1 of appendix $\mathrm{B}$ show the relative contribution of unreconstructed final states to these transitions with hadronic mass less than $1.0 \mathrm{GeV} / c^{2}$ in $\mathrm{MC}$ data. In total $50.1 \%$ of the final states are not reconstructed. Correcting for this gives an extrapolated BF of $[3.68 \pm 0.14$ (stat.) \pm 0.20 (sys.) $] \times 10^{-5}$. As the decays of $K^{*}$ mesons are well known [14] no systematic uncertainty is applied due to this extrapolation.

The MC model of $B \rightarrow X_{d}^{L} \gamma$ data only considers strong decays of $\rho$ mesons which correspond to reconstructed modes. The decay model of $\omega$ mesons does contain contributions from unreconstructed final states, a summary of which is given table B.3 of appendix B for MC data with hadronic mass less than $1.0 \mathrm{GeV} / c^{2}$. In total, after weighting the relative contributions of $\left(\rho^{+}: \rho^{0}: \omega\right)$ to world average measurements, $2.0 \%$ of final states are not reconstructed. Correcting for these gives an extrapolated BF of $[0.99 \pm 0.27$ (stat. $) \pm 0.14($ sys. $)] \times 10^{-6}$. The dominant $\omega \rightarrow \pi^{+} \pi^{-} \pi^{0}$ transition has an associated uncertainty of $0.8 \%$ [14]; however, propagating this as a systematic error in the 


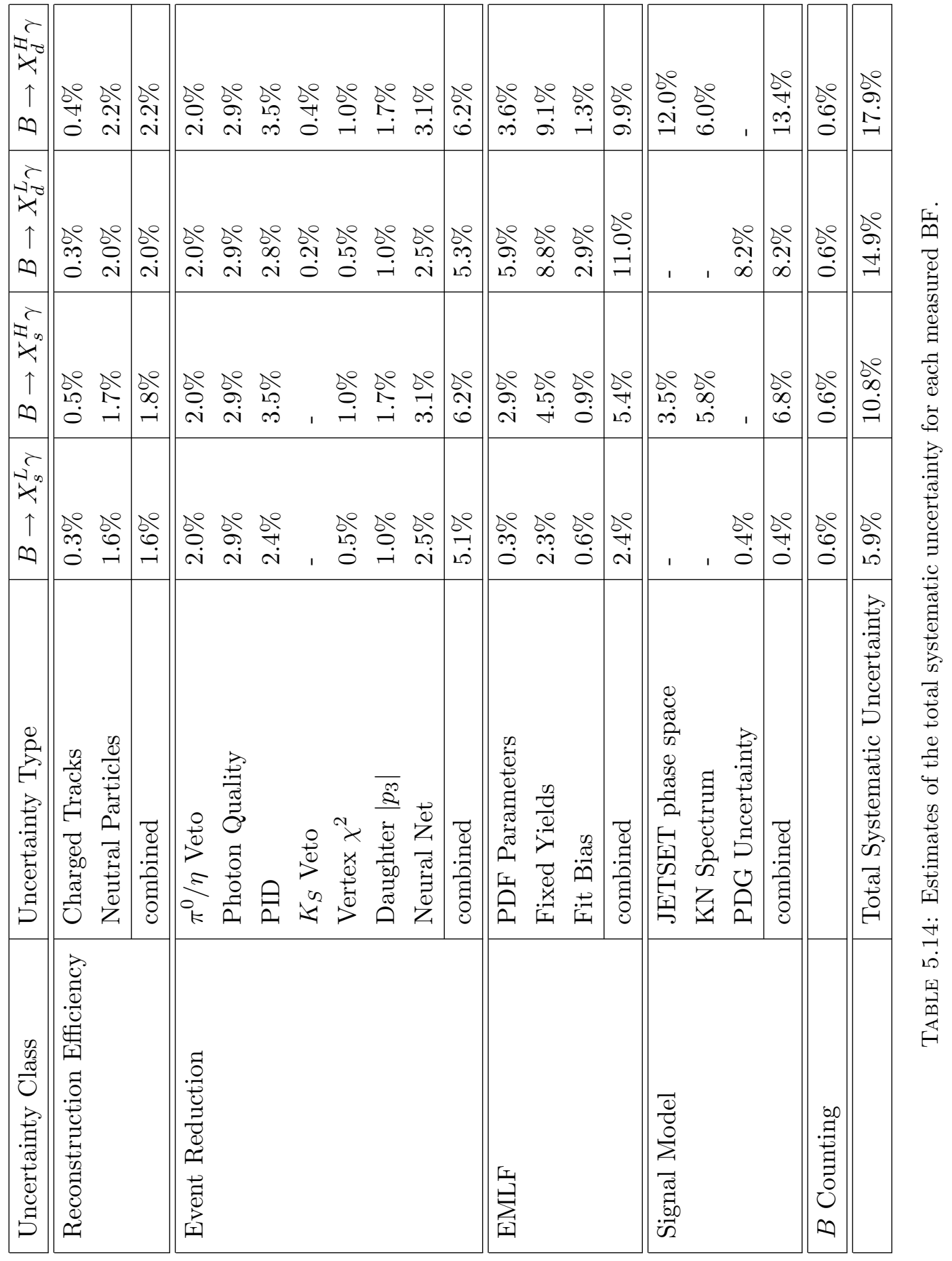




\begin{tabular}{|l|l|}
\hline Fit & Measured BF \\
\hline$B \rightarrow X_{s}^{L} \gamma$ & $(1.84 \pm 0.07 \pm 0.11) \times 10^{-5}$ \\
$B \rightarrow X_{s}^{H} \gamma$ & $(6.30 \pm 0.24 \pm 0.68) \times 10^{-5}$ \\
$B \rightarrow X_{d}^{L} \gamma$ & $(0.97 \pm 0.27 \pm 0.14) \times 10^{-6}$ \\
$B \rightarrow X_{d}^{H} \gamma$ & $(3.60 \pm 0.92 \pm 0.64) \times 10^{-6}$ \\
\hline
\end{tabular}

TABLE 5.15: Measured BF estimation showing associated statistical error for each fit to experimental data and the corresponding estimate of systematic uncertainty before BF extrapolation.

extrapolation shows it has a negligible contribution to the overall systematic uncertainty and so it is not considered further.

\subsubsection{High Mass Region BF Extrapolation}

Measured BFs with hadronic mass in the range $1.0 \leq m_{X} \leq 2.0 \mathrm{GeV} / c^{2}$ are used to estimate the total $\mathrm{BF}$ of $B \rightarrow X_{(s, d)} \gamma$ transitions with mass greater than $1.0 \mathrm{GeV} / c^{2}$. This extrapolation is made in two steps, firstly a total $\mathrm{BF}$ in the measured mass range is estimated and then the $\mathrm{KN}$ model is used to infer the correction required for unreconstructed states with mass greater than $2.0 \mathrm{GeV} / c^{2}$.

\subsubsection{Correction for Final States with $1.0 \leq m_{X} \leq 2.0 \mathrm{GeV} / c^{2}$}

The total number of events in the mass range $1.0 \leq m_{X} \leq 2.0 \mathrm{GeV} / c^{2}$ is determined by the KN model parameterisation. With the default MC models (weighted for $B \rightarrow X_{s}^{H} \gamma$ data and as generated for $B \rightarrow X_{d}^{H} \gamma$ data) the correction is simply determined by the ratio of the total number of signal events to all events in the mass region. For $B \rightarrow X_{s}^{H} \gamma$ MC data $35.4 \%$ of events in this mass range are signal modes giving an extrapolated $\mathrm{BF}$ of $[17.8 \pm 0.7$ (stat. $) \pm 1.9$ (sys.) $] \times 10^{-5}$. The corresponding signal proportion in $B \rightarrow X_{d}^{H} \gamma \mathrm{MC}$ data is $42.3 \%$ of events in the mass range which gives extrapolated $\mathrm{BF}$ $[8.5 \pm 2.2$ (stat. $) \pm 1.5$ (sys.) $] \times 10^{-6}$. However, the proportion of signal events in the mass range is not necessarily well modelled due to uncertainties in the JETSET phase space decay model so further systematic uncertainties must be applied to the extrapolated BFs to account for this.

In section 5.1.4.2 varying the relative contributions of signal modes was used to determine a systematic uncertainty in the signal efficiency. In these alternative models the total number of signal events were not constant. It follows that, assuming the model changes do not significantly alter the results of fits to data, these variations result in a different extrapolated BF as well as a different measured BF. The errors must therefore 


\begin{tabular}{|l|l|}
\hline Signal Mode & Uncertainty \\
\hline$B^{0} \rightarrow K^{+} \pi^{-} \gamma$ & $1.8 \%$ \\
$B^{0} \rightarrow K^{+} \pi^{0} \gamma$ & $1.2 \%$ \\
$B^{0} \rightarrow K^{+} \pi^{-} \pi^{+} \gamma$ & $2.9 \%$ \\
$B^{0} \rightarrow K^{+} \pi^{-} \pi^{0} \gamma$ & $1.5 \%$ \\
$B^{0} \rightarrow K^{+} \pi^{-} \pi^{+} \pi^{-} \gamma$ & $0.6 \%$ \\
$B^{0} \rightarrow K^{+} \pi^{-} \pi^{+} \pi^{0} \gamma$ & $0.9 \%$ \\
$B^{0} \rightarrow K^{+} \eta \gamma$ & $1.4 \%$ \\
\hline All Signal & $4.3 \%$ \\
\hline
\end{tabular}

TABLE 5.16: Systematic uncertainties due to uncertainties in the weights applied to $B \rightarrow X_{s}^{H} \gamma$ signal MC data modes after BF extrapolation in the signal mass range. These values modify those given in table 5.10 as potentially correlated variations in the overall proportion of signal events within the mass range are accounted for.

be propagated for the extrapolated BF accounting for this effect. Table 5.16 lists the average variation in the extrapolated BFs obtained from the existing calculated uncertainty for each $B \rightarrow X_{s}^{H} \gamma$ signal mode. These are then combined in quadrature to give an overall corrected systematic uncertainty of $4.3 \%$ for the extrapolated BF. When propagating the alternative $B \rightarrow X_{d}^{H} \gamma$ signal model systematic uncertainty, the relative proportion of signal events in the new model is $45.0 \%$ and the measured BF due to the efficiency change is $4.09 \times 10^{-6}$. This therefore results in an extrapolated BF which varies by $6.9 \%$ from the default value. This is assigned as the corrected systematic for the extrapolated $\mathrm{BF}$ of these data.

Further uncertainties in the proportion of signal events within the signal mass region arise from the modelled distribution of unreconstructed modes the relative contributions of which are not well known. These are classified into two categories, those with 2-4 bodies in the final state and those with 5 or more bodies in the final state. The previous version of this analysis investigated the contribution from such states by engineering alternative fragmentation models of the hadronic system and seeing how the proportion of those unreconstructed classes change relative to the default model [1]. Models considered are applied to both $X_{s}$ and $X_{d}$ MC data and include the default JETSET fragmentation, the application of weights measured in previous $B \rightarrow X_{s} \gamma$ experimental datasets (see section 4.2) and a hybrid model of exclusive resonant MC data with the JETSET events. The hybrid model uses the resonant decays listed in table 5.17; the resonant decays are assigned half of the the total inclusive width in this region with the other half remain JETSET decays. Each resonance is assumed to contribute equally. The variations in unknown modes under these alternative models corresponds to no more than $50 \%$ for either category, hence this can be considered a conservative limit. The previous analysis study motivates assigning a systematic uncertainty on the extrapolated 


\begin{tabular}{|l|l|}
\hline$B \rightarrow X_{s}^{H} \gamma$ & $B \rightarrow X_{d}^{H} \gamma$ \\
\hline$B \rightarrow K_{1}(1270) \gamma$ & $B \rightarrow h_{1}(1170) \gamma$ \\
$B \rightarrow K_{1}(1400) \gamma$ & $B \rightarrow b_{1}(1235) \gamma$ \\
$B \rightarrow K^{*}(1410) \gamma$ & $B \rightarrow a_{1}(1260) \gamma$ \\
$B \rightarrow K_{2}^{*}(1430) \gamma$ & $B \rightarrow f_{2}^{0}(1270) \gamma$ \\
$B \rightarrow K^{*}(1680) \gamma$ & $B \rightarrow f_{1}^{0}(1285) \gamma$ \\
& $B \rightarrow a_{2}^{0}(1320) \gamma$ \\
\hline
\end{tabular}

TABLE 5.17: Resonant decays used to construct the hybrid fragmentation model investigated in the previous version of this analysis [1].

BF whereby unreconstructed contributions in the default models are varied individually by $\pm 50 \%$. The uncertainty for a given unknown category is calculated by varying one unknown mode while keeping the relative contribution of signal and the remaining unknown mode constant, but renormalising them relative to the varied mode to maintain overall unity in the mass range. The resulting variation in the signal contribution then forms the systematic uncertainty; if the variation is asymmetric the larger value is taken. By keeping the other unknown mode constant relative to the signal in a given variation the uncertainties from each unknown class are to a good approximation independent and hence their sum in quadrature gives the overall uncertainty due to unreconstructed final states from JETSET decays.

The distribution of unreconstructed states in $B \rightarrow X_{s}^{H} \gamma \mathrm{MC}$ data do not include decays corresponding to a signal mode where the $K^{+}$final state meson is substituted for a $K^{0}$ meson. This is because such decays exhibit an isospin symmetry with the signal modes and are therefore constrained by that symmetry. In the signal mass range the proportion of signal modes is $35.4 \%$, corresponding $K^{0}$ modes $36.4 \%$, other $2-4$ body modes $16.0 \%$ and 5 or more body modes $12.2 \%$. Varying the $2-4$ body mode proportion by $\pm 50 \%$ gives an average relative change in the extrapolated $\mathrm{BF}$ of $9.6 \%$. The corresponding variation of 5 or more body modes gives a change of $7.0 \%$. An overall systematic uncertainty due to unreconstructed modes is therefore taken to be $11.9 \%$. Accounting for this and the above corrected signal JETSET uncertainty the extrapolated BF in the signal mass range for $B \rightarrow X_{s}^{H} \gamma$ data is therefore $[17.8 \pm 0.7$ (stat. $) \pm 2.9$ (sys.) $] \times 10^{-5}$.

Unreconstructed states in $B \rightarrow X_{d}^{H} \gamma \mathrm{MC}$ data are all varied as none are expected to be constrained by the signal modes. In the signal mass range for these data the proportion of signal modes is $42.3 \%$, other $2-4$ body modes $27.0 \%$ and 5 or more body modes $30.7 \%$. The uncertainty obtained from varying the $2-4$ body mode proportion by $\pm 50 \%$ is $19.2 \%$ and the corresponding uncertainty due to 5 or more body modes is $23.3 \%$. This gives an overall systematic uncertainty due to unreconstructed modes of $30.2 \%$. Correcting the systematic error to account for the above extrapolation uncertainties gives 
the extrapolated $\mathrm{BF}$ in the signal mass range for $B \rightarrow X_{d}^{H} \gamma$ data as $[8.5 \pm 2.2$ (stat.) \pm 2.9 (sys.) $] \times 10^{-6}$.

\subsubsection{Correction for Final States with $m_{X}>2.0 \mathrm{GeV} / c^{2}$}

Given the extrapolated BF values calculated in the hadronic mass range $1.0 \leq m_{X} \leq$ $2.0 \mathrm{GeV} / c^{2}$, estimates of the total BF for all masses greater than $1.0 \mathrm{GeV} / c^{2}$ are made for both $B \rightarrow X_{s}^{H} \gamma$ and $B \rightarrow X_{d}^{H} \gamma$ data. The generated signal MC data using the default KN model parameterisation shows that for all masses greater than $1.0 \mathrm{GeV} / c^{2}$ in $B \rightarrow X_{s}^{H} \gamma$ data, $60.2 \%$ of events are generated with $1.0 \leq m_{X} \leq 2.0 \mathrm{GeV} / c^{2}$. Given this proportion the total $\mathrm{BF}$ with mass greater than $1.0 \mathrm{GeV} / c^{2}$ is calculated to be $[29.5 \pm 1.1$ (stat. $) \pm 4.8$ (sys.) $] \times 10^{-5}$. For $B \rightarrow X_{d}^{H} \gamma$ data the corresponding proportion of events with $1.0 \leq m_{X} \leq 2.0 \mathrm{GeV} / c^{2}$ is also $60.2 \%$ which gives the total BF with mass greater than $1.0 \mathrm{GeV} / c^{2}$ as $[14.2 \pm 3.6$ (stat.) $\pm 4.8($ sys. $)] \times 10^{-6}$. However, the $\mathrm{KN}$ model contribution to the systematic uncertainties in the above BFs must be corrected to account for uncertainties in the extrapolation due to the parameterisation of this model. For each transition flavour the complete high mass extrapolation is repeated assuming alternative KN parameterisations. The efficiencies given in table 5.8 are used to revise the measured $\mathrm{BF}$ calculation assuming no change in the result of the fit to experimental data. The BFs for all hadronic masses greater than $1.0 \mathrm{GeV} / c^{2}$ are then recalculated using the relevant proportions of signal candidates under the alternative KN scheme. The average variation in the full $\mathrm{BF}$ for $B \rightarrow X_{s}^{H} \gamma$ data is $1.6 \%$ and the corresponding variation in $B \rightarrow X_{d}^{H} \gamma$ data is $1.9 \%$. These are therefore assigned as the corrected systematic uncertainties due to different parameterisations of the $\mathrm{KN}$ model. With this correction applied the the total BFs for transitions with hadronic mass greater than $1.0 \mathrm{GeV} / c^{2}$ is $[29.5 \pm 1.1$ (stat.) \pm 4.5 (sys.) $] \times 10^{-5}$ for $B \rightarrow X_{s}^{H} \gamma$ data and $[14.2 \pm 3.6$ (stat. $) \pm 4.7$ (sys.) $] \times 10^{-6}$ for $B \rightarrow X_{d}^{H} \gamma$ data.

\subsubsection{Summary of Extrapolated BFs}

Table 5.18 summarises the extrapolated BFs calculated for each mass region. For the high mass region both the extrapolated $\mathrm{BF}$ in the hadronic mass range $1.0 \leq m_{X} \leq 2.0 \mathrm{GeV} / c^{2}$ and the extrapolated BF for all hadronic masses with $1.0 \mathrm{GeV} / c^{2} \leq m_{X}$ are shown. The full extrapolated BFs in the high and low mass regions are not directly combined into an overall inclusive BF due to correlations in the systematic uncertainties which are discussed below. 


\begin{tabular}{|l|l|l|}
\hline Fit & Hadronic Mass Range & Extrapolated BF \\
\hline \hline$B \rightarrow X_{s}^{L} \gamma$ & $<1.0 \mathrm{GeV} / c^{2}$ & $(3.68 \pm 0.14 \pm 0.20) \times 10^{-5}$ \\
$B \rightarrow X_{s}^{H} \gamma$ & $1.0-2.0 \mathrm{GeV} / c^{2}$ & $(17.8 \pm 0.7 \pm 2.9) \times 10^{-5}$ \\
& $\geq 1.0 \mathrm{GeV} / c^{2}$ & $(29.5 \pm 1.1 \pm 4.5) \times 10^{-5}$ \\
\hline$B \rightarrow X_{d}^{L} \gamma$ & $<1.0 \mathrm{GeV} / c^{2}$ & $(0.99 \pm 0.27 \pm 0.14) \times 10^{-6}$ \\
$B \rightarrow X_{d}^{H} \gamma$ & $1.0-2.0 \mathrm{GeV} / c^{2}$ & $(8.5 \pm 2.2 \pm 2.9) \times 10^{-6}$ \\
& $\geq 1.0 \mathrm{GeV} / c^{2}$ & $(14.2 \pm 3.6 \pm 4.7) \times 10^{-6}$ \\
\hline
\end{tabular}

TABLE 5.18: Extrapolated BF estimation showing the associated statistical error for each fit to experimental data and the corresponding estimate of systematic uncertainty which has been recalculated to account for uncertainties in the extrapolation method.

\subsubsection{Calculation of $\left|V_{t d} / V_{t s}\right|$ and Associated Uncertainty}

The evaluation of $R$ is given by the relation,

$$
R=\frac{\mathcal{B}\left(B \rightarrow X_{d} \gamma\right)^{m_{x}<1.0 \mathrm{GeV} / c^{2}}+\mathcal{B}\left(B \rightarrow X_{d} \gamma\right)^{m_{x}>1.0 \mathrm{GeV} / c^{2}}}{\mathcal{B}\left(B \rightarrow X_{s} \gamma\right)^{m_{x}<1.0 \mathrm{GeV} / c^{2}}+\mathcal{B}\left(B \rightarrow X_{s} \gamma\right)^{m_{x}>1.0 \mathrm{GeV} / c^{2}}}
$$

Using the extrapolated BFs calculated above therefore gives $R=\left(15.14 \times 10^{-6}\right) /(33.22 \times$ $\left.10^{-5}\right)=0.0456$. The corresponding uncertainties in $R$ must take account any expected correlations in the contributions to the uncertainties on each extrapolated BF. There are four possible correlations for each uncertainty. Firstly no correlation in the uncertainty; in this case the average variation in $R$ must be calculated separately for the variation due to each individual $\mathrm{BF}$ and these contributions then added in quadrature. Secondly the BFs corresponding to the same flavour transition have a correlated uncertainty; in this case the the average variation in $R$ due to each flavour transition is calculated and these are added in quadrature. Thirdly the BFs corresponding to the same mass range have a correlated uncertainty; similarly the average variation in $R$ due to each mass range is calculated and these are added in quadrature. Finally the uncertainty is correlated for all BFs; in this case the average variation in $R$ due to all BFs is calculated. The propagation of the overall PID systematic is calculated as the linear combination of the individual charged pion and charged kaon PID contributions to the uncertainty in $R$. This is due to potential correlations in the input variables for each PID selector. The charged pion PID uncertainty in $R$ is correlated for all BFs and calculated to be $1.2 \%$. The charged kaon PID contribution is also correlated for all BFs (although $B \rightarrow X_{d} \gamma$ BFs have no contribution from this uncertainty) and calculated to give a 1.1\% uncertainty in $R$. Table 5.19 list all sources of uncertainty calculated for the individual BFs, indicates their expected correlation and evaluates the corresponding uncertainty on $R$. Combining 


\begin{tabular}{|l|l|l|}
\hline Uncertainty Type & Correlation Type & $R$ Uncertainty \\
\hline Fit; Statistical & None & $24.2 \%$ \\
\hline Track Reconstruction & All & $0.1 \%$ \\
Neutral Reconstruction & All & $0.5 \%$ \\
Photon Cuts & All & $0.0 \%$ \\
Combined PID & All & $2.3 \%$ \\
$K_{S}$ Veto & Flavour & $0.4 \%$ \\
Vertex $\chi^{2}$ & Mass Range & $0.1 \%$ \\
Daughter $\left|p_{3}\right|$ & Mass Range & $0.1 \%$ \\
Neural Net & Mass Range & $0.1 \%$ \\
Fit; PDF Fixed Parameters & None & $4.3 \%$ \\
Fit; Fixed $B \rightarrow X_{s}^{L} \gamma$ XF Yield & All & $0.5 \%$ \\
Fit; Fixed $B \rightarrow X_{s}^{H} \gamma$ XF Yield & All & $0.0 \%$ \\
Fit; Fixed $B \rightarrow X_{d}^{L} \gamma$ XF Yield & Flavour & $0.6 \%$ \\
Fit; Fixed $B \rightarrow X_{d}^{H} \gamma$ XF Yield & Flavour & $1.9 \%$ \\
Fit; Fixed PID XF Yield & Flavour & $1.2 \%$ \\
Fit; Fixed Generic $B$ Yields & All & $5.6 \%$ \\
Fit; Floated Parameter Bias & None & $1.5 \%$ \\
JETSET Model Signal Modes & None & $7.5 \%$ \\
JETSET Model Unreconstructed 2-4 Body Modes & Mass Range & $9.5 \%$ \\
JETSET Model Unreconstructed 5+ Body Modes & Mass Range & $15.6 \%$ \\
KN Spectrum & Mass Range & $0.4 \%$ \\
PDG Uncertainty & None & $0.5 \%$ \\
$B$ Counting & All & $0.0 \%$ \\
\hline Combined Systematic & - & $21.3 \%$ \\
\hline
\end{tabular}

TABLE 5.19: Estimated uncertainties in the calculation of $R$. There are four correlation types. 'All' represents the case where all four extrapolated BFs are varied simultaneously. 'Flavour' represents the case where BFs of the same flavour are varied simultaneously and the resulting uncertainties in $R$ combined in quadrature. 'Mass Range' represents the case where BFs in the same mass region are varied simultaneously and the resulting uncertainties in $R$ combined in quadrature. 'None' represents the case where each $\mathrm{BF}$ is varied independently and the resulting uncertainties in $R$ combined in quadrature.

these individual uncertainty contributions in quadrature gives,

$$
R=0.0456 \pm 0.0110(\text { stat. }) \pm 0.0097 \text { (sys.) }
$$

Solving (1.67) for the calculated value of $R$ gives,

$$
\left|\frac{V_{t d}}{V_{t s}}\right|=0.211 \pm 0.023(\text { stat. }) \pm 0.022(\text { sys. }) \pm 0.001(\text { th. }),
$$


where the final uncertainty is the associated theoretical error estimated numerically from the variations shown in figure 1.9. This value is in good agreement with the world average value from $B$ mixing experiments which is $0.209 \pm 0.006$ [14]. The systematic uncertainty of this measurement is dominated by the uncertainties due to the JETSET phase space model, particularly in extrapolating the measured BFs to inclusive values. The systematic and statistical uncertainties are of a similar order. 


\section{Chapter 6}

\section{Summary and Future Prospects}

The analysis presented uses experimental data recorded with the BABAR detector to measure the sum of seven exclusive $B \rightarrow X_{s} \gamma$ and $B \rightarrow X_{d} \gamma$ decay BFs in the hadronic mass ranges $0.5 \leq m_{X}<1.0 \mathrm{GeV} / c^{2}$ and $1.0 \leq m_{X} \leq 2.0 \mathrm{GeV} / c^{2}$. For each mass range the event selection has been optimised with simulated data to maximise the signal yield of CKM-suppressed $B \rightarrow X_{d} \gamma$ transitions over their associated combinatoric backgrounds. Identical event selection has been applied to $B \rightarrow X_{s} \gamma$ transitions as to their $B \rightarrow X_{d} \gamma$ counterparts. Signal yields in experimental data have been extracted with unbinned extended maximum likelihood fits and interpreted as BF measurements. Comprehensive evaluation of the systematic uncertainties relating to these measurements have been made. Table 6.1 lists the measured BF for each transition flavour in each mass range and their associated uncertainties. The measured $B \rightarrow X_{d} \gamma$ BF in the mass range $1.0 \leq m_{X} \leq 2.0 \mathrm{GeV} / c^{2}$ represents the first statistically significant signal yield of such transitions with hadronic mass above the $\rho / \omega$ resonant peaks.

The measured BFs have been extrapolated in a model dependent way to estimate BFs for all $B \rightarrow X_{s} \gamma$ and $B \rightarrow X_{d} \gamma$ transitions in the hadronic mass ranges $m_{X}<1.0 \mathrm{GeV} / c^{2}$ and $m_{X} \geq 1.0 \mathrm{GeV} / c^{2}$. Evaluations of the associated systematic uncertainties due to the extrapolation technique have been made. Table 6.2 lists the extrapolated BF for each transition flavour in each mass range and their associated uncertainties. The existing world average values of these measurements from [14] are shown in table 6.3. Allowing

\begin{tabular}{|l|l|l|}
\hline & $0.5 \leq m_{X}<1.0 \mathrm{GeV} / c^{2}$ & $1.0 \leq m_{X} \leq 2.0 \mathrm{GeV} / c^{2}$ \\
\hline$B \rightarrow X_{s} \gamma$ & $(1.84 \pm 0.07 \pm 0.11) \times 10^{-5}$ & $(6.30 \pm 0.24 \pm 0.68) \times 10^{-5}$ \\
$B \rightarrow X_{d} \gamma$ & $(0.97 \pm 0.27 \pm 0.14) \times 10^{-6}$ & $(3.60 \pm 0.92 \pm 0.64) \times 10^{-6}$ \\
\hline
\end{tabular}

TABLE 6.1: Summary of measured BFs. In each case the first error is statistical and the second systematic. 


\begin{tabular}{|l|l|l|}
\hline & $m_{X}<1.0 \mathrm{GeV} / c^{2}$ & $1.0 \leq m_{X} \mathrm{GeV} / c^{2}$ \\
\hline$B \rightarrow X_{s} \gamma$ & $(3.68 \pm 0.14 \pm 0.20) \times 10^{-5}$ & $(29.5 \pm 1.1 \pm 4.5) \times 10^{-5}$ \\
$B \rightarrow X_{d} \gamma$ & $(0.99 \pm 0.27 \pm 0.14) \times 10^{-6}$ & $(14.2 \pm 3.6 \pm 4.7) \times 10^{-6}$ \\
\hline
\end{tabular}

TABLE 6.2: Summary of extrapolated BFs. In each case the first error is statistical and the second systematic.

\begin{tabular}{|l|l|}
\hline Mode & BF from [14] \\
\hline$B \rightarrow K^{*} \gamma$ & $(4.2 \pm 0.6) \times 10^{-5}$ \\
$\bar{b} \rightarrow \bar{s} \gamma$ & $(35.6 \pm 2.5) \times 10^{-5}$ \\
$B \rightarrow \rho / \omega \gamma$ & $(1.3 \pm 0.2) \times 10^{-6}$ \\
\hline
\end{tabular}

TABLE 6.3: Existing world average BF measurements.

\begin{tabular}{|l|l|}
\hline Measurement & $\left|V_{t d} / V_{t s}\right|$ \\
\hline Inclusive Penguin & $0.211 \pm 0.032$ \\
Exclusive Penguin [14] & $0.210 \pm 0.040$ \\
$B$ Mixing [14] & $0.209 \pm 0.006$ \\
\hline
\end{tabular}

TABLE 6.4: $\left|V_{t d} / V_{t s}\right|$ measurement comparisons.

for the fact that $10 \%$ of $K^{*}$ and $\rho$ resonance peaks have hadronic mass greater than $1.0 \mathrm{GeV} / c^{2}$ is can be seen that the extrapolated BFs are in good agreement with the world average values.

The extrapolated BFs are combined to give a ratio of the $B \rightarrow X_{d} \gamma$ transition to the $B \rightarrow X_{s} \gamma$ transition of $R(d \gamma / s \gamma)=0.0456 \pm 0.0110$ (stat.) \pm 0.0097 (sys.). This parameter is interpreted using its theoretical relation to the CKM parameters $(\bar{\rho}, \bar{\eta})$ presented in [22]. After transforming the basis of this relation to the orthogonal coordinates $\left(\left|V_{t d} / V_{t s}\right|, \beta\right)$, discussed in section 1.2.2.2, the calculated value of $R(d \gamma / s \gamma)$ is found to correspond to $\left|V_{t d} / V_{t s}\right|=0.211 \pm 0.023$ (stat.) \pm 0.022 (sys.) \pm 0.001 (th.) where the final error corresponds to the uncertainty in the theoretical relation. The extracted value of $\left|V_{t d} / V_{t s}\right|$ is compared to previous measurements in table 6.4 ; this table essentially summarises the measurements discussed in section 1.3. It is found to be entirely consistent and has uncertainties comparable to those from exclusive radiative penguin measurements; furthermore it makes a significant improvement compared the previous version of this analysis [1]. The agreement of both this and the exclusive radiative penguin measurement with the value extracted from $B$ mixing suggests no significant new physics amplitude due to the operator $C_{7}(\mu) Q_{7}$ which exclusively contributes to calculations of the radiative penguin processes. 


\subsection{Future Prospects}

This measurement is performed on the complete $B A B A R$ experimental dataset collected at the $\Upsilon(4 S)$ resonance and so is unlikely to be improved by the BABAR collaboration. However, there is an ongoing $B A B A R$ analysis to update the $B \rightarrow X_{s} \gamma$ measurement from [30] using the full experimental dataset. The systematic uncertainty due to model dependancies in $B \rightarrow X_{s}^{H} \gamma$ MC data could be significantly reduced using the results of such an analysis, although these do not dominate the total systematic uncertainty calculated here.

Other future prospects for improving the measurement of $\left|V_{t d} / V_{t s}\right|$ from radiative penguin decays are threefold. Firstly the BELLE experiment at KEK in Japan [72] also has a significant volume of $e^{+} e^{-}$collision data recorded at the $\Upsilon(4 S)$ resonance, the collection of which is ongoing at the time of writing. These data could well be used to perform an analysis similar to the one presented here, reducing the statistical uncertainties due to $B \rightarrow X_{d} \gamma$ transitions. However, the measurement is unlikely to improve the modelling uncertainties due to JETSET. Secondly the LHCb experiment at CERN [73] has started to record proton-proton collision data with the aim of measuring processes from both $B$ and $B_{s}$ meson decays. The noisier environment of hadronic collisions makes the prospect of measuring $\left|V_{t d} / V_{t s}\right|$ from inclusive decays unlikely, although studies suggest a competitive measurement from exclusive decays is possible and this forms an important part of their physics program [74]. However, such a measurement from exclusive decays will suffer from the larger theoretical uncertainties discussed in section 1.2.2.3. Finally proposals exist for a next generation of high luminosity $e^{+} e^{-}$collision experiments dedicated to data collection at the $\Upsilon(4 S)$ resonance $[75,76]$. The proposal in [75] uses a detector design heavily based on the BABAR experiment and in fact proposes to reuse components of the now dismantled BABAR detector. These designs project potential datasets in excess of $30 \mathrm{ab}^{-1}$ which could potentially make the $B \rightarrow X_{d} \gamma$ transition measurements as competitive as existing $B \rightarrow X_{s} \gamma$ measurements. It follows that a measurement of $\left|V_{t d} / V_{t s}\right|$ similar to the measurement presented here could be made with much reduced statistical error. If this were to be the case such a measurement would also have to undertake significant studies to reduce the dominant systematic uncertainties. Datasets of this size could well be used to measure a significantly larger proportion of $B \rightarrow X_{d} \gamma$ decay modes at a relatively small cost in statistical power (as demonstrated when modes eight and nine of table 3.1 were investigated here). This would reduce the modelling uncertainty; however the increased contribution of combinatoric backgrounds from generic $B$ decays would also need extensive study as that was shown to be a significant contribution to the systematic uncertainty here. In any case these high luminosity experiments, if approved, are unlikely to begin data collection 
before 2015. Significant improvement of the measurement presented here is therefore unlikely in the near future.

\subsection{Addendum}

The analysis presented in this thesis has been modified by the BABAR collaboration, with assistance from the author, and was submitted to Physical Review Letters on 21st May 2010 [77]. The modifications to the methods presented in this thesis are briefly summarised.

Event selection is unmodified and the MC and experimental datasets used in MLFs are identical. The fits to data are significantly modified. The generic $B$ normalisation is allowed to float at a cost in fit bias on the signal yield. However, the additional bias is significantly less than the systematic uncertainties associated with fixed normalisations for this background class which were calculated here. In addition the weights for measured $B \rightarrow X_{s} \gamma$ modes are evaluated from fits to the high mass $B \rightarrow X_{s} \gamma$ experimental data. They are generally found to be consistent with weights from the previous version of this analysis, but do modify the the signal efficiency of the $B \rightarrow X_{s}^{H} \gamma$ measurement and thus give a different $\mathrm{BF}$; this also modifies the signal model systematic uncertainty calculated when the weights are applied to $B \rightarrow X_{d}^{H} \gamma \mathrm{MC}$ data. Furthermore the submitted analysis assumes isospin holds for $\rho$ and $\omega$ resonant decays, whereas the analysis here uses the central value of previous exclusive radiative penguin measurements. This sightly modifies the measured and extrapolated $B \rightarrow X_{d}^{L} \gamma$ BF. The evaluation of the NN systematic uncertainty is modified but still evaluated with the high statistics $B \rightarrow X_{s} \gamma$ experimental data. The NN systematic is used to correct the efficiency in MC data which is not done here. This results in different signal efficiencies and hence measured BFs for all experimental data. The BF extrapolation is not significantly modified; however, an additional alternative JETSET fragmentation model is engineered whereby only low mass scalar mesons are allowed. This does not increase the $\pm 50 \%$ variation observed previously in unreconstructed modes. The published analysis finds $\left|V_{t d} / V_{t s}\right|=0.199 \pm 0.022 \pm 0.024 \pm 0.002[77]$ where the first error is statistical, the second error systematic and third error theoretical. The theoretical error is slightly larger as it corresponds to a different value of the ratio $R$. This result varies from the measurement in this thesis by $\sim 5 \%$; the variation is entirely consistent with the systematic difference in the fit method and the modified NN systematic in the published analysis. 


\section{Appendix A}

\section{Monomial Functions and Sphericity Tensor Variable Distributions}

This appendix contains plots of the monomial functions and sphericity tensor variables discussed in sections 3.3.2.2 and 3.3.2.3 respectively. In all cases the solid line shows the distribution for correctly reconstructed signal candidates before event selection cuts are applied and the dashed line shows the corresponding distribution for continuum backgrounds.

\section{A.1 Monomial Function Distributions}

Figures A.1-A.3 show the longitudinal monomials for the ROE with respect to the thrust axis of the ROE calculated in the CM frame. Note that $L_{1}^{T}$ is identical to the thrust of the ROE. Figures A.4-A.6 show the perpendicular monomials for the ROE with respect to the thrust axis of the ROE calculated in the CM frame. Figures A.7-A.9 show the longitudinal monomials for the ROE with the momentum axis of the high energy photon used in candidate reconstruction calculated in the CM frame. Figures A.10-A.12 show the perpendicular monomials for the ROE with the momentum axis of the high energy photon used in candidate reconstruction calculated in the CM frame. 


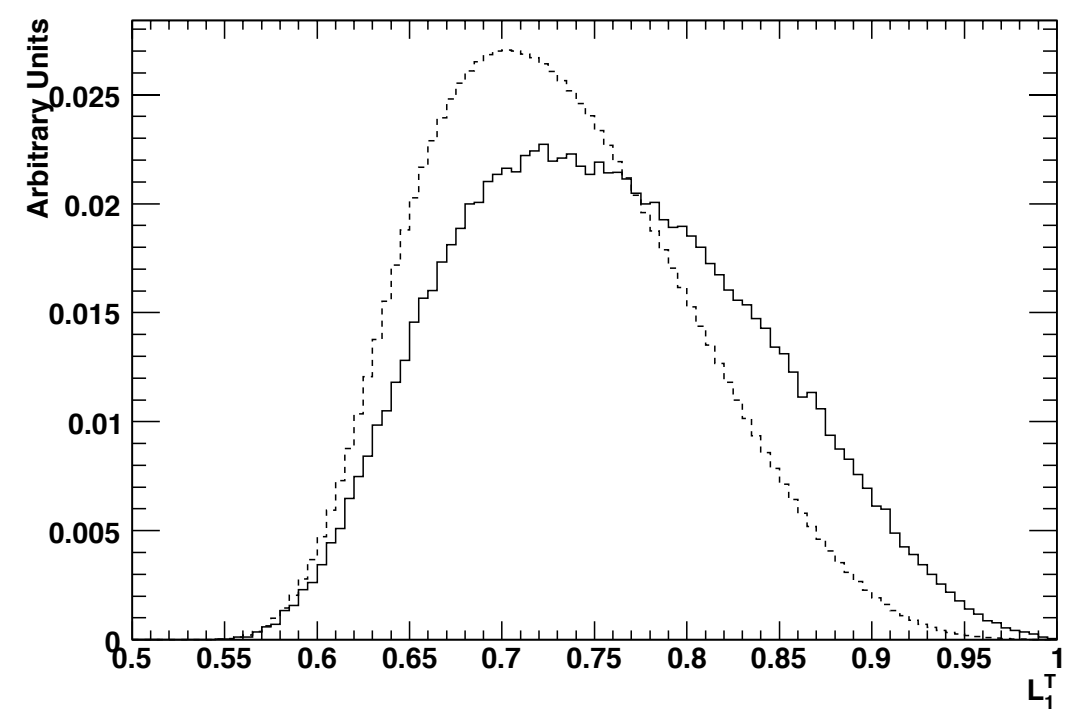

Figure A.1: Distributions of the monomial $L_{1}^{T}$

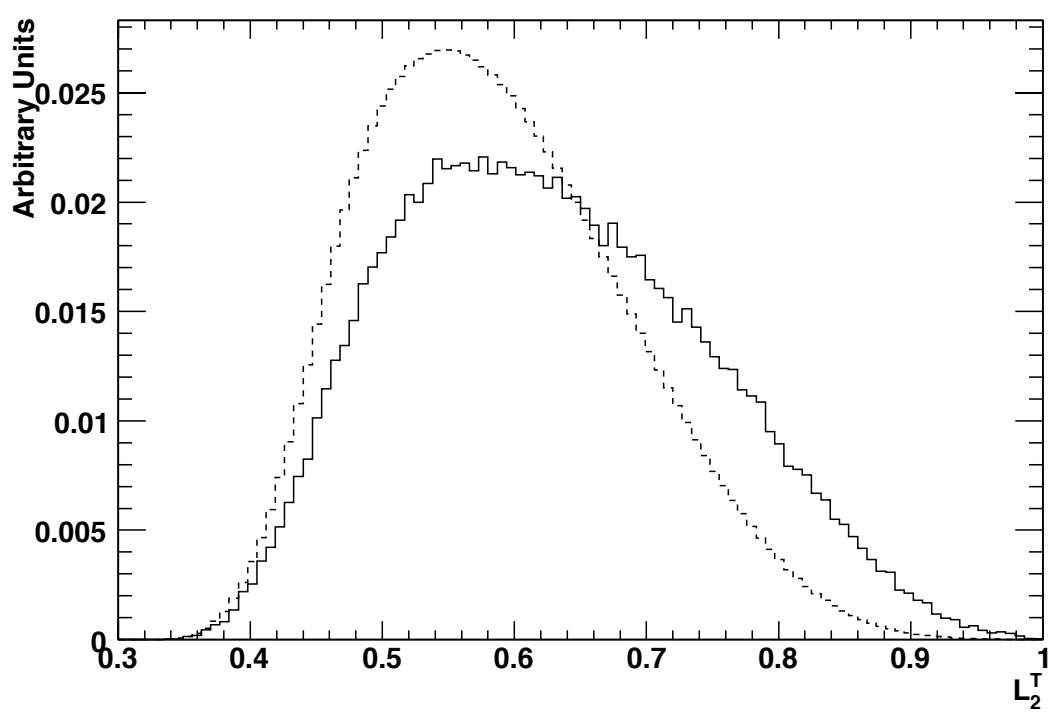

Figure A.2: Distributions of the monomial $L_{2}^{T}$ 


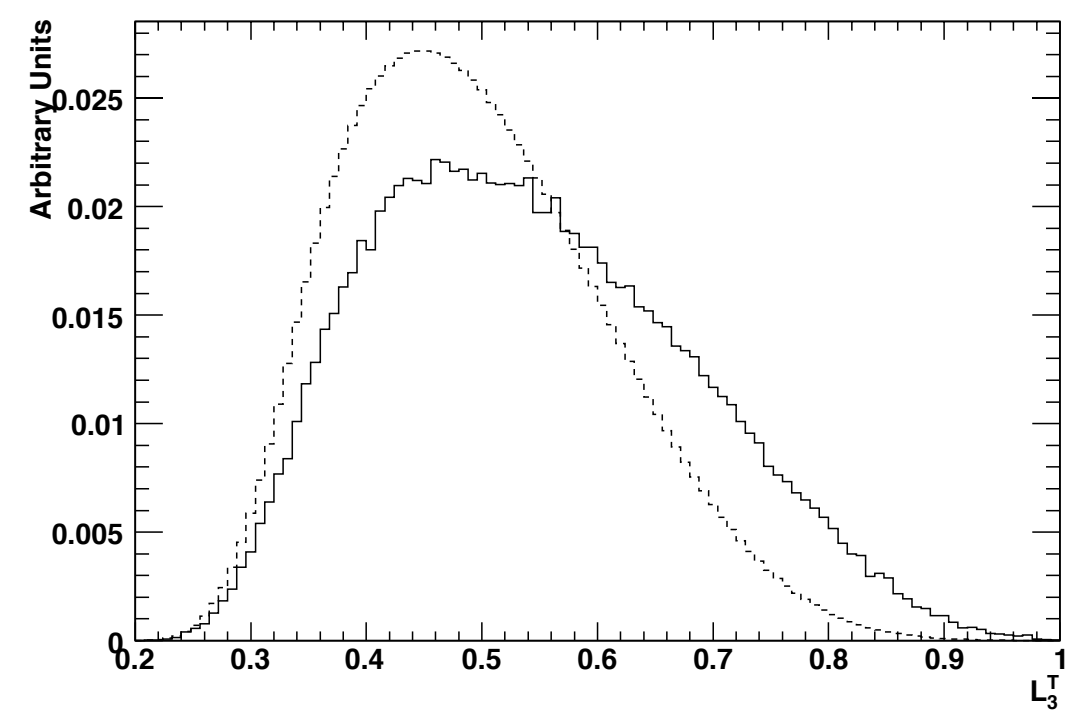

Figure A.3: Distributions of the monomial $L_{3}^{T}$

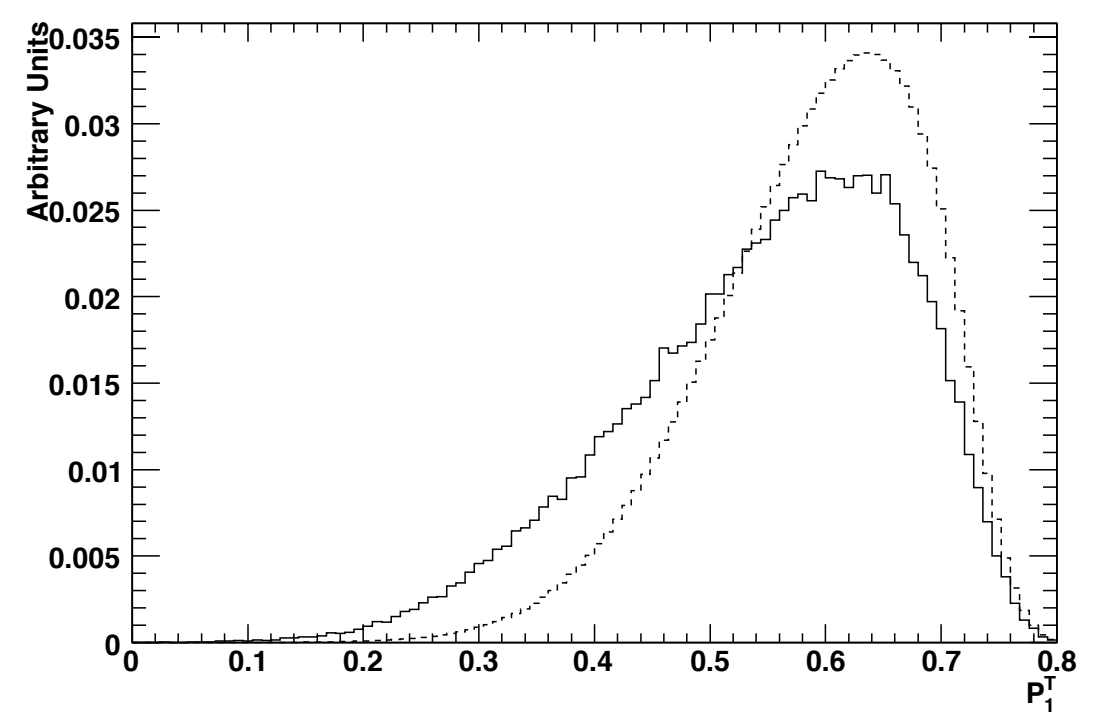

Figure A.4: Distributions of the monomial $P_{1}^{T}$ 


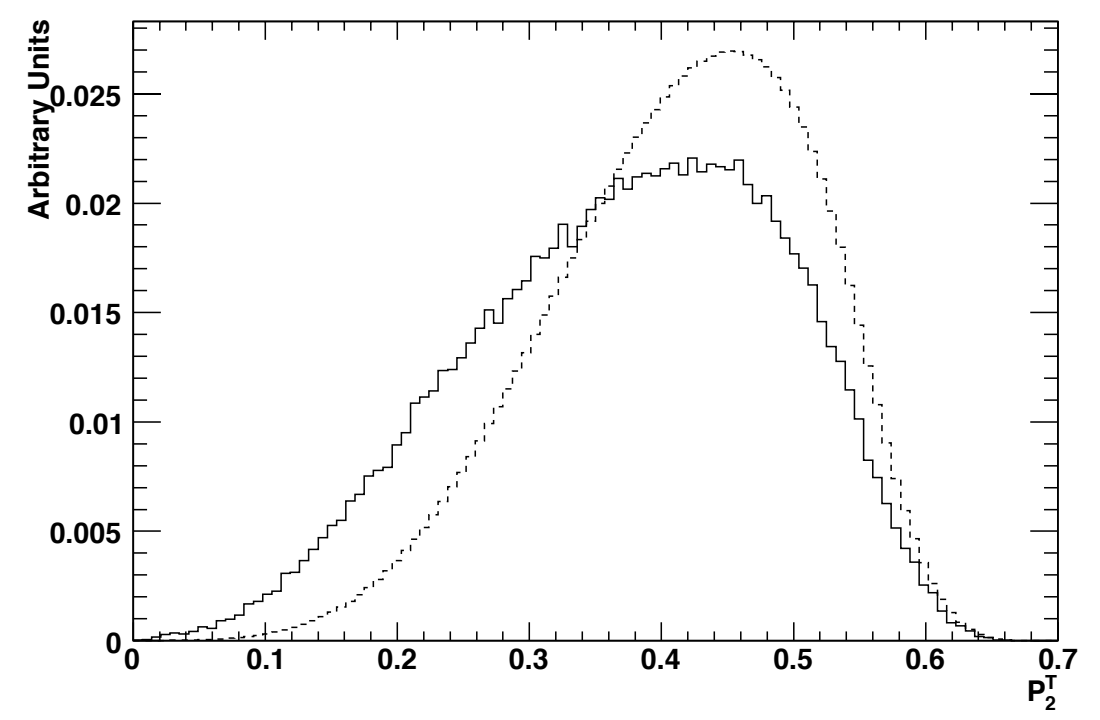

Figure A.5: Distributions of the monomial $P_{2}^{T}$

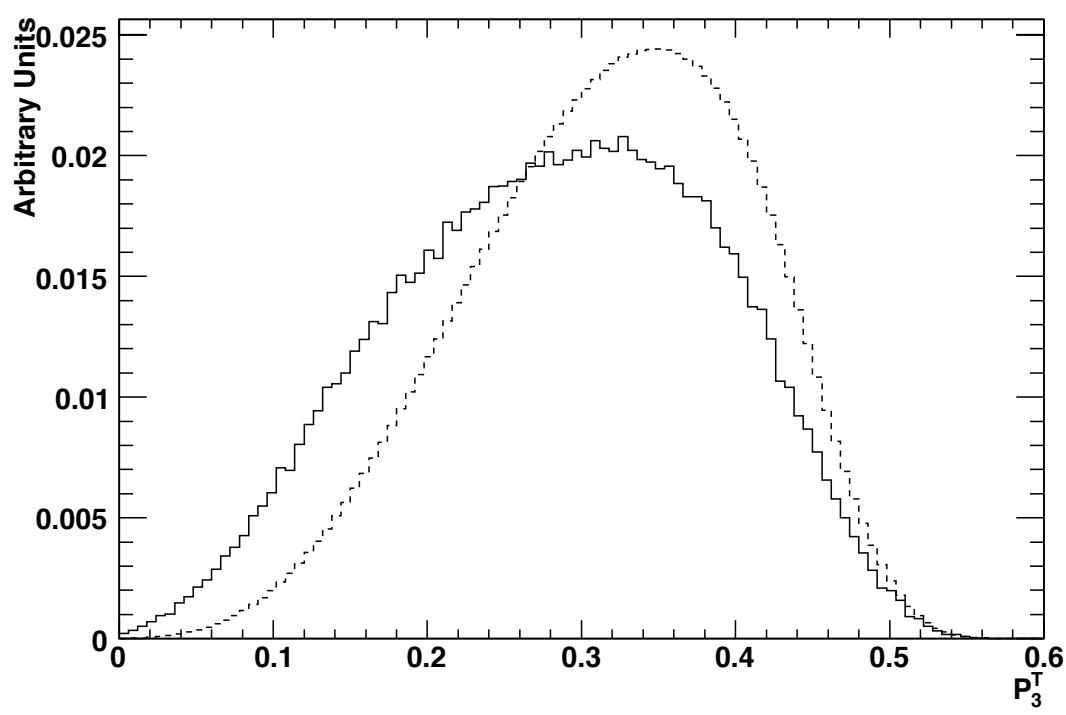

Figure A.6: Distributions of the monomial $P_{3}^{T}$ 


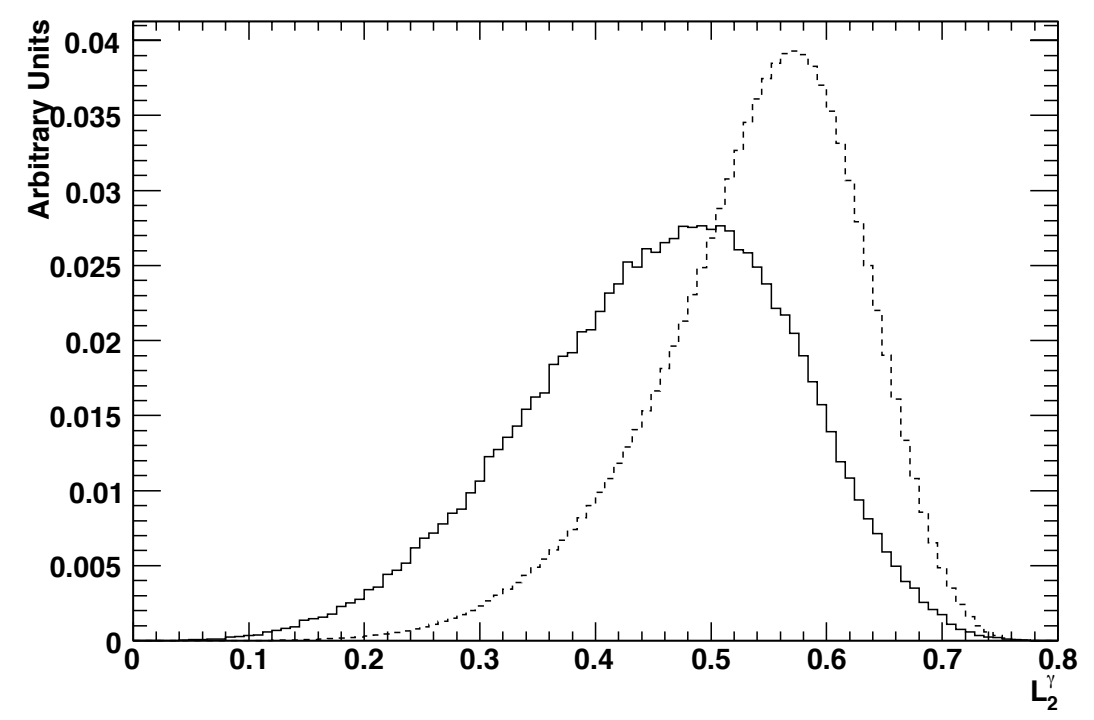

FIgURE A.7: Distributions of the monomial $L_{1}^{\gamma}$

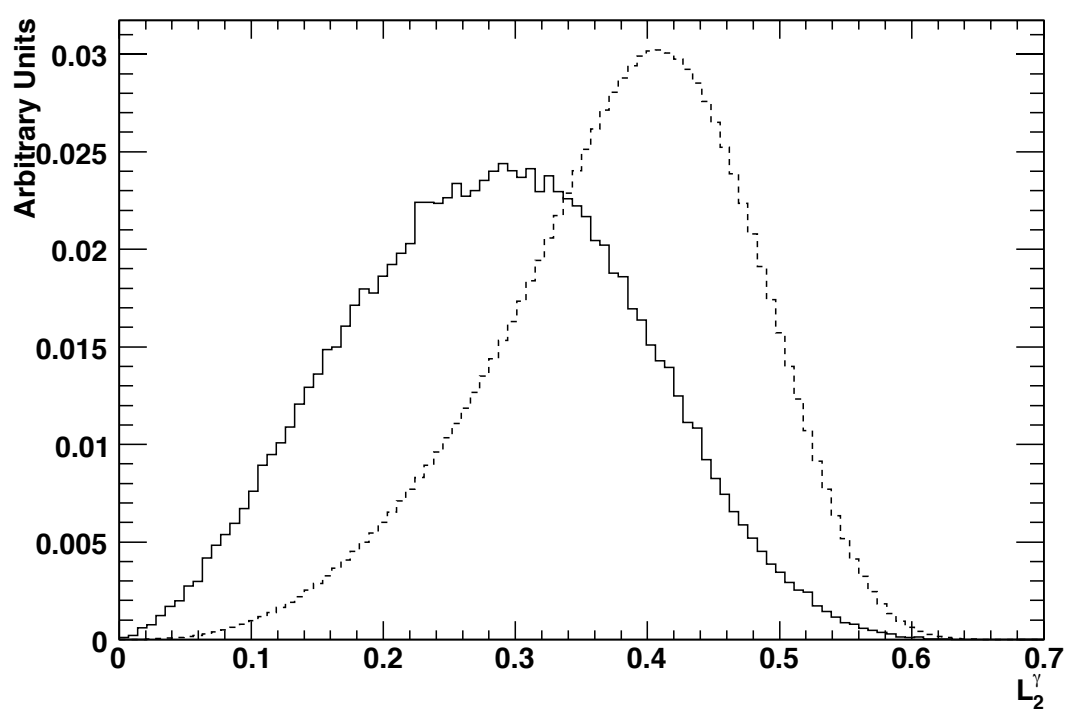

Figure A.8: Distributions of the monomial $L_{2}^{\gamma}$ 


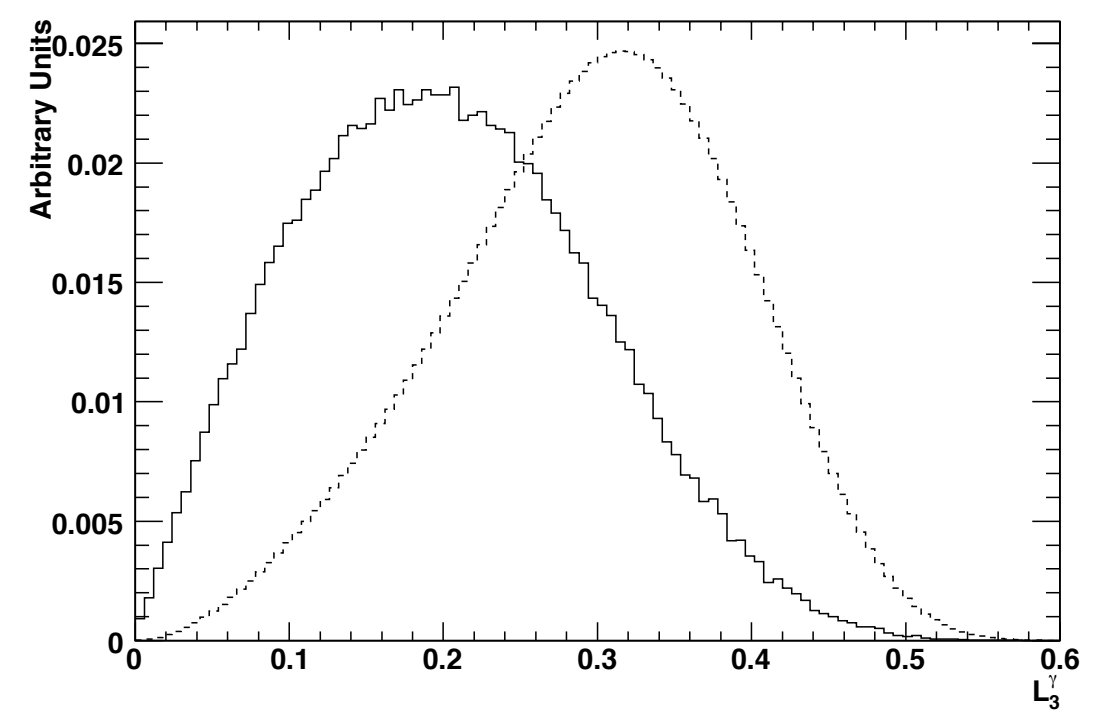

Figure A.9: Distributions of the monomial $L_{3}^{\gamma}$

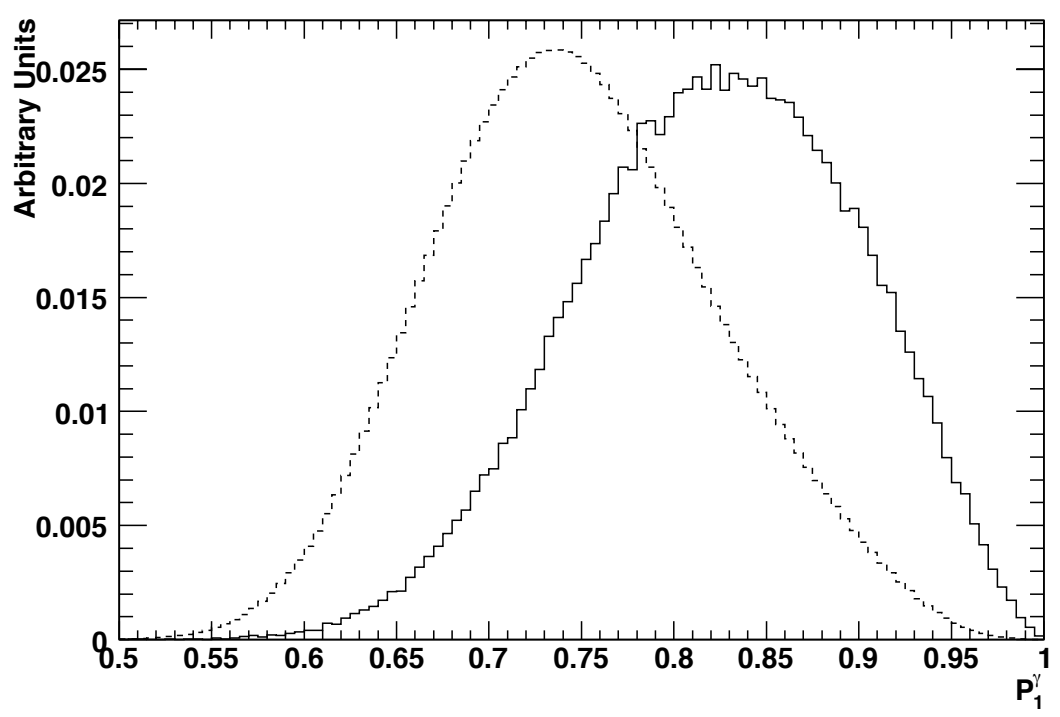

Figure A.10: Distributions of the monomial $P_{1}^{\gamma}$ 


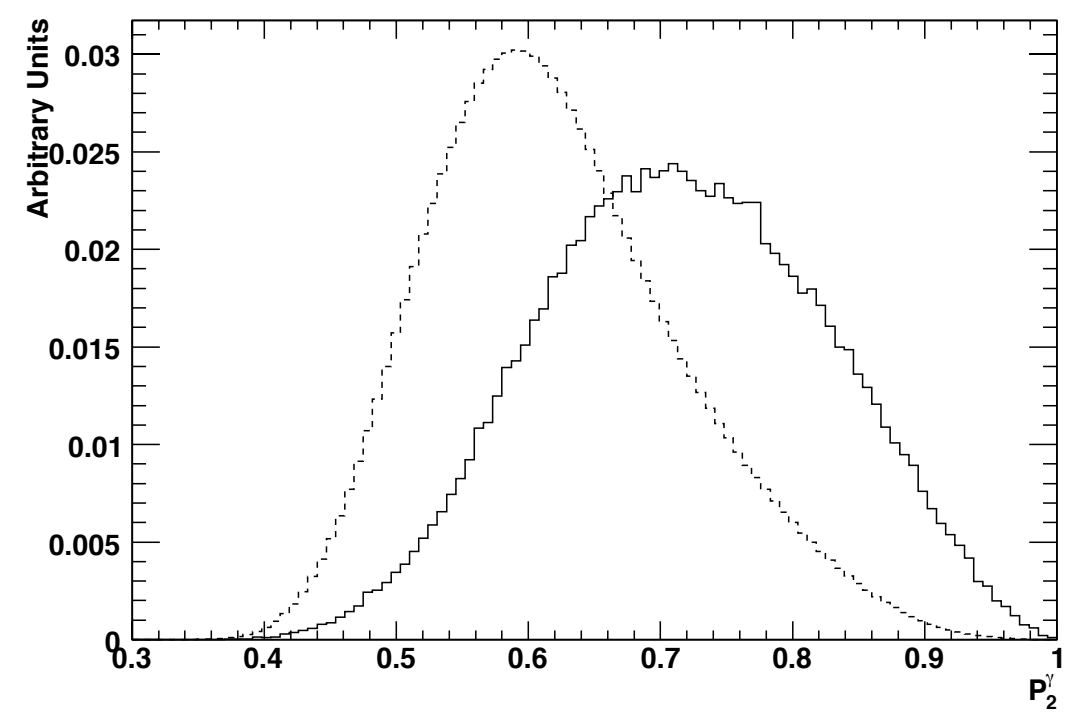

Figure A.11: Distributions of the monomial $P_{2}^{\gamma}$

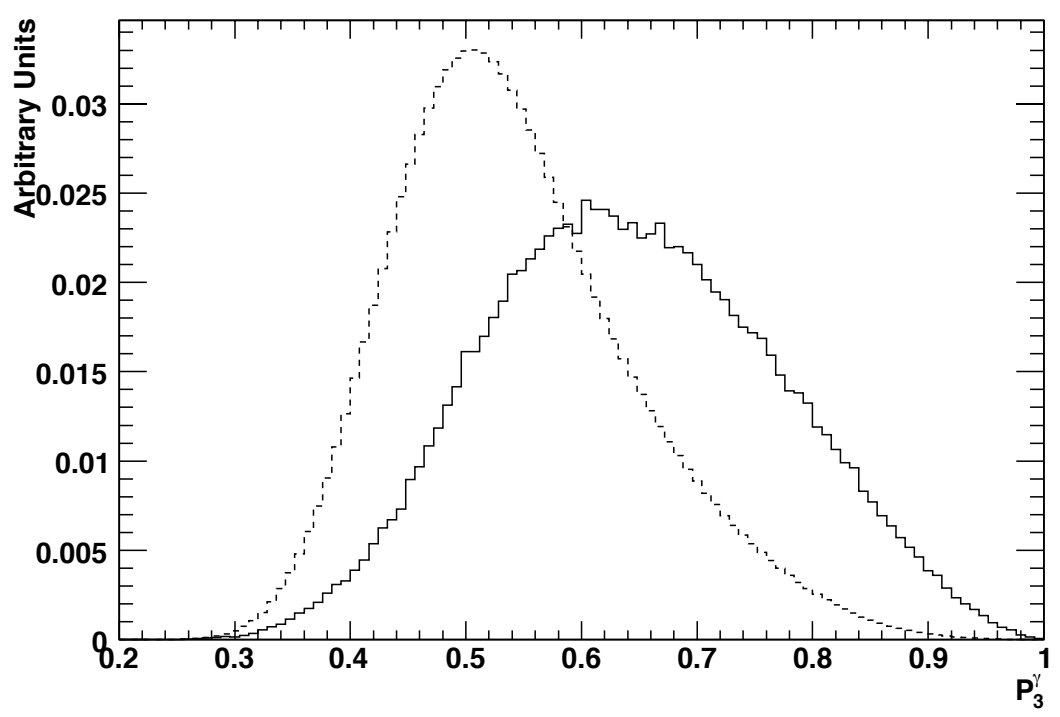

Figure A.12: Distributions of the monomial $P_{2}^{\gamma}$ 


\section{A.2 Sphericity Tensor Variable Distributions}

Figure A.13 shows the sphericity of the ROE, figure A.14 shows the planarity of the ROE and A.15 shows the aplanarity of the ROE.

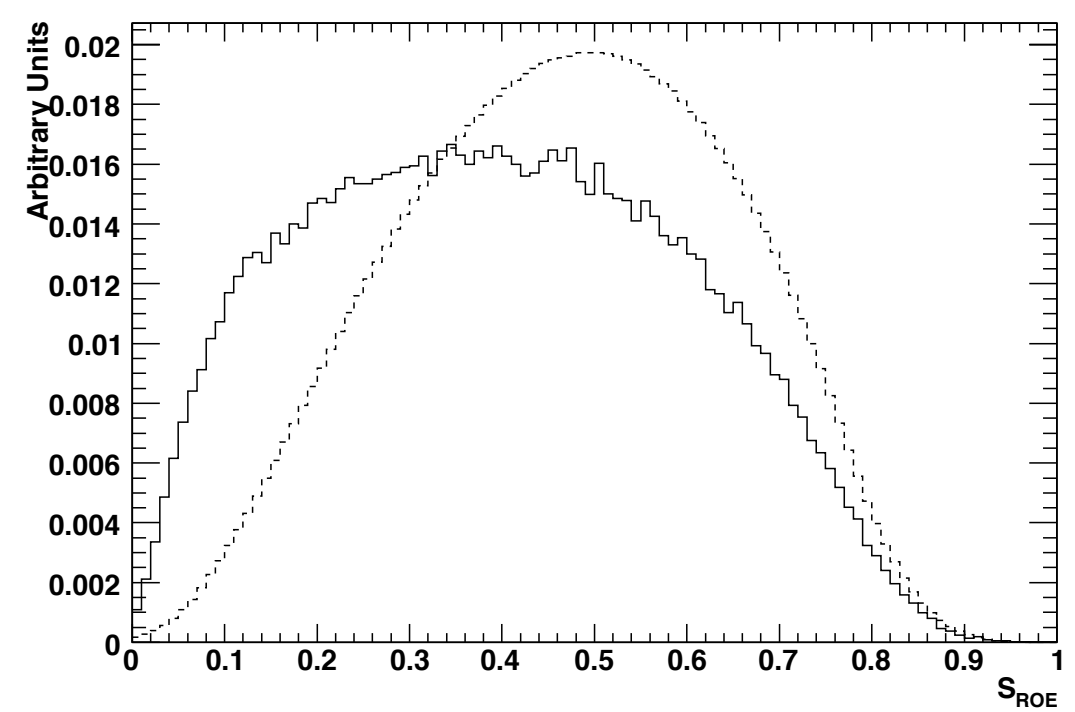

Figure A.13: Distributions of $S_{R O E}$

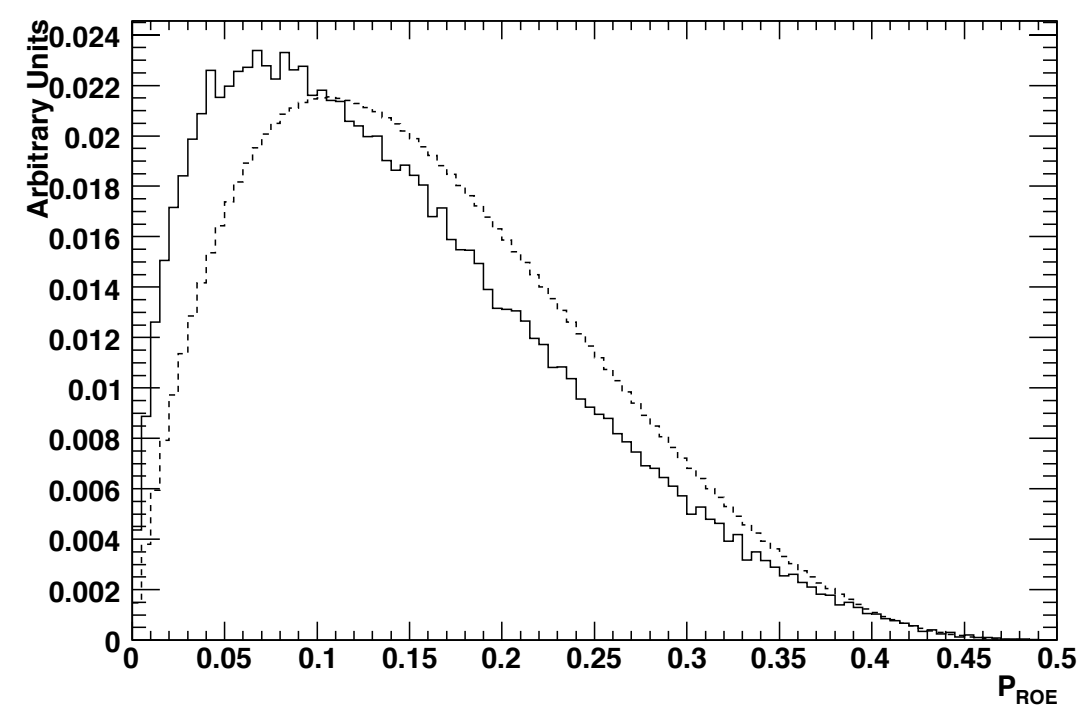

Figure A.14: Distributions of $P_{R O E}$ 


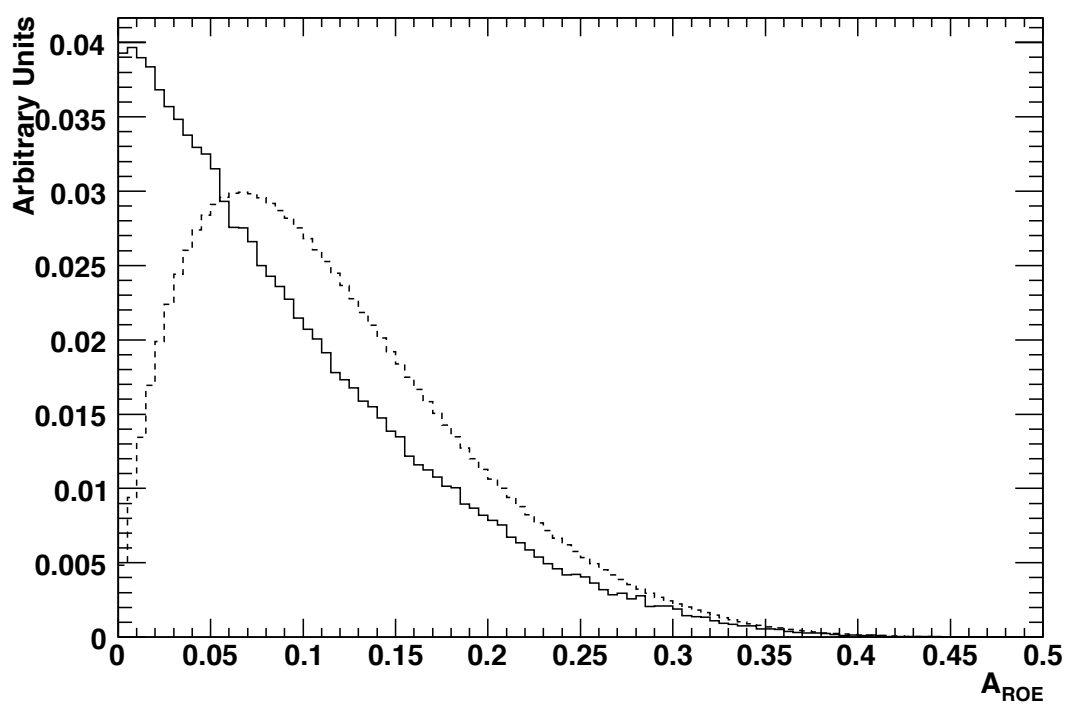

Figure A.15: Distributions of $A_{R O E}$ 


\section{Appendix B}

\section{Signal Monte Carlo Data Event Classification}

This appendix contains tables summarising the distributions of hadronic final states in the generated signal MC data. Where a particular final state corresponds to one of the reconstructed modes listed in table 3.1 this is indicated numerically in parentheses after that mode.

\section{B.1 Resonant MC data}

\section{B.1.1 $B \rightarrow X_{s} \gamma$}

Tables B.1 and B.2 list the distribution of final states for $B^{+} \rightarrow K^{*+} \gamma$ and $B^{0} \rightarrow K^{* 0} \gamma$ respectively. These distributions are determined by the measured decays of the $K^{*}$ resonance [14].

\begin{tabular}{|l|l|}
\hline Mode & Hadronic Mass $<1.0 \mathrm{GeV} / c^{2}$ \\
\hline$B^{+} \rightarrow K^{*+}\left(K^{0} \pi^{+}\right) \gamma$ & $3,827,841$ \\
$B^{+} \rightarrow K^{*+}\left(K^{+} \pi^{0}\right) \gamma(2)$ & $1,918,199$ \\
Other 2 body $K^{*+}$ & 6,131 \\
\hline Total & $5,752,171$ \\
\hline
\end{tabular}

TABLE B.1: Distribution of final states in $B^{+} \rightarrow K^{*+} \gamma$ signal MC data 


\begin{tabular}{|l|l|}
\hline Mode & Hadronic Mass $<1.0 \mathrm{GeV} / c^{2}$ \\
\hline$B^{0} \rightarrow K^{* 0}\left(K^{+} \pi^{-}\right) \gamma(1)$ & $3,814,990$ \\
$B^{0} \rightarrow K^{* 0}\left(K^{0} \pi^{0}\right) \gamma$ & $1,900,490$ \\
Other 2 body $K^{* 0}$ & 11,993 \\
\hline Total & $5,727,473$ \\
\hline
\end{tabular}

TABLE B.2: Distribution of final states in $B^{0} \rightarrow K^{* 0} \gamma$ signal MC data

\begin{tabular}{|l|l|}
\hline Mode & Hadronic Mass $<1.0 \mathrm{GeV} / c^{2}$ \\
\hline$B^{0} \rightarrow \omega\left(\pi^{+} \pi^{-}\right) \gamma(1)$ & 15,738 \\
$B^{0} \rightarrow \omega\left(\pi^{+} \pi^{-} \pi^{0}\right) \gamma(4)$ & 576,785 \\
$B^{0} \rightarrow \omega\left(\pi^{+} \pi^{-} \pi^{+} \pi^{-}\right) \gamma(5)$ & 596 \\
Other 2 body $\omega$ & 55,967 \\
Other 3 body $\omega$ & 914 \\
\hline Total & 650,000 \\
\hline
\end{tabular}

TABLE B.3: Distribution of final states in $B^{0} \rightarrow \omega \gamma$ signal MC data

\section{B.1.2 $\quad B \rightarrow X_{d} \gamma$}

Table B.3 lists the distribution of final states in $B^{0} \rightarrow \omega \gamma \mathrm{MC}$ data. This distribution is determined by the measured decays of the $\omega$ resonance [14]. The distributions of final states for $B \rightarrow \rho \gamma$ modes are not shown as the MC data assumes all $\rho^{+} \rightarrow \pi^{+} \pi^{0}$ and all $\rho^{0} \rightarrow \pi^{+} \pi^{-}$.

\section{B.2 Non-Resonant MC data with Generated Hadronic Mass Following the KN Model}

\section{B.2.1 $\quad B \rightarrow X_{s} \gamma$}

Tables B.4 and B.5 show the distribution of final states for high mass signal MC data for $B^{+} \rightarrow X_{s}^{+} \gamma$ and $B^{0} \rightarrow X_{s}^{0} \gamma$ decays respectively. The hadronic mass is determined from the $\mathrm{KN}$ model and the final state distributions are determined by phase space decays from JETSET [44]. The total number of decays are shown as well as the number with generated hadronic mass within the mass range of reconstructed candidates. The application of weights to these data, as described in section 4.2, is shown in table B.6. 


\begin{tabular}{|l|l|l|}
\hline Mode & Hadronic Mass $\geq 1.0 \mathrm{GeV} / c^{2}$ & Hadronic Mass $1.0-2.0 \mathrm{GeV} / c^{2}$ \\
\hline$B^{+} \rightarrow K^{0} \pi^{+} \gamma$ & 970,100 & 856,693 \\
$B^{+} \rightarrow K^{+} \pi^{0} \gamma(2)$ & 595,196 & 526,122 \\
$B^{+} \rightarrow K^{+} \pi^{+} \pi^{-} \gamma(3)$ & $1,098,686$ & 915,113 \\
$B^{+} \rightarrow K^{0} \pi^{+} \pi^{0} \gamma$ & $1,332,026$ & $1,110,800$ \\
$B^{+} \rightarrow K^{+} \pi^{0} \pi^{0} \gamma(8)$ & 271,142 & 226,589 \\
$B^{+} \rightarrow K^{0} \pi^{+} \pi^{-} \pi^{+} \gamma$ & 391,333 & 255,914 \\
$B^{+} \rightarrow K^{+} \pi^{+} \pi^{-} \pi^{0} \gamma(6)$ & $1,004,779$ & 701,015 \\
$B^{+} \rightarrow K^{0} \pi^{+} \pi^{0} \pi^{0} \gamma$ & 346,501 & 227,603 \\
$B^{+} \rightarrow K^{+} \pi^{+} \pi^{-} \pi^{+} \pi^{-} \gamma$ & 139,007 & 61,728 \\
$B^{+} \rightarrow K^{0} \pi^{+} \pi^{-} \pi^{+} \pi^{0} \gamma$ & 613,763 & 315,611 \\
$B^{+} \rightarrow K^{+} \pi^{+} \pi^{-} \pi^{0} \pi^{0} \gamma$ & 397,167 & 196,662 \\
$B^{+} \rightarrow K^{+} \eta \gamma(7)$ & 85,037 & 74,348 \\
$B^{+} \rightarrow K^{0} \pi^{+} \eta \gamma$ & 63,897 & 46,157 \\
$B^{+} \rightarrow K^{+} \pi^{0} \eta \gamma$ & 34,520 & 24,968 \\
$B^{+} \rightarrow K^{+} \pi^{+} \pi^{-} \eta \gamma$ & 35,901 & 19,172 \\
$B^{+} \rightarrow K^{0} \pi^{+} \pi^{0} \eta \gamma$ & 37,407 & 19,274 \\
$B^{+} \rightarrow K^{+} K^{-} K^{+} \gamma$ & 60,945 & 40,808 \\
$B^{+} \rightarrow K^{0} K^{+} K^{-} \pi^{+} \gamma$ & 55,037 & 18,265 \\
$B^{+} \rightarrow K^{+} K^{-} K^{+} \pi^{0} \gamma$ & 29,800 & 10,259 \\
Other 2 body & 30 & 0 \\
Other 3 body & 93,615 & 51,192 \\
Other 4 body & 233,179 & 137,447 \\
Other $5+$ body & $2,536,332$ & 445,731 \\
\hline Total & $10,425,400$ & $6,281,471$ \\
\hline
\end{tabular}

TABle B.4: Distribution of final states in high mass $B^{+} \rightarrow X_{s}^{+} \gamma$ signal MC data. The hadronic mass is determined by the KN model.

\section{B.2.2 $\quad B \rightarrow X_{d} \gamma$}

Tables B.7 and B.8 show the distribution of final states for high mass signal MC data for $B^{+} \rightarrow X_{d}^{+} \gamma$ and $B^{0} \rightarrow X_{d}^{0} \gamma$ decays respectively. The hadronic mass is determined from the $\mathrm{KN}$ model and the final state distributions are determined by phase space decays from JETSET [44]. The total number of decays are shown as well as the number with generated hadronic mass within the mass range of reconstructed candidates. 


\begin{tabular}{|l|l|l|}
\hline Mode & Hadronic Mass $\geq 1.0 \mathrm{GeV} / c^{2}$ & Hadronic Mass $1.0-2.0 \mathrm{GeV} / c^{2}$ \\
\hline$B^{0} \rightarrow K^{+} \pi^{-} \gamma(1)$ & 971,350 & 858,131 \\
$B^{0} \rightarrow K^{0} \pi^{0} \gamma$ & 594,227 & 524,904 \\
$B^{0} \rightarrow K^{0} \pi^{+} \pi^{-} \gamma$ & $1,104,038$ & 919,640 \\
$B^{0} \rightarrow K^{+} \pi^{-} \pi^{0} \gamma(4)$ & $1,334,309$ & $1,112,561$ \\
$B^{0} \rightarrow K^{0} \pi^{0} \pi^{0} \gamma$ & 268,178 & 224,300 \\
$B^{0} \rightarrow K^{+} \pi^{-} \pi^{+} \pi^{-} \gamma(5)$ & 393,695 & 256,414 \\
$B^{0} \rightarrow K^{0} \pi^{+} \pi^{-} \pi^{0} \gamma$ & $1,036,398$ & 725,643 \\
$B^{0} \rightarrow K^{+} \pi^{-} \pi^{0} \pi^{0} \gamma(9)$ & 347,271 & 228,359 \\
$B^{0} \rightarrow K^{0} \pi^{+} \pi^{-} \pi^{+} \pi^{-} \gamma$ & 141,785 & 63,083 \\
$B^{0} \rightarrow K^{+} \pi^{-} \pi^{+} \pi^{-} \pi^{0} \gamma$ & 569,578 & 289,191 \\
$B^{0} \rightarrow K^{0} \pi^{+} \pi^{-} \pi^{0} \pi^{0} \gamma$ & 407,614 & 203,538 \\
$B^{0} \rightarrow K^{0} \eta \gamma$ & 85,063 & 74,155 \\
$B^{0} \rightarrow K^{+} \pi^{-} \eta \gamma$ & 63,890 & 46,109 \\
$B^{0} \rightarrow K^{0} \pi^{0} \eta \gamma$ & 34,088 & 24,576 \\
$B^{0} \rightarrow K^{0} \pi^{+} \pi^{-} \eta \gamma$ & 39,168 & 21,004 \\
$B^{0} \rightarrow K^{+} \pi^{-} \pi^{0} \eta \gamma$ & 37,538 & 19,185 \\
$B^{0} \rightarrow K^{0} K^{+} K^{-} \gamma$ & 61,209 & 41,026 \\
$B^{0} \rightarrow K^{+} K^{-} K^{+} \pi^{-} \gamma$ & 34,781 & 12,085 \\
$B^{0} \rightarrow K^{0} K^{+} K^{-} \pi^{0} \gamma$ & 29,613 & 10,024 \\
Other 2 body & 29 & 0 \\
Other 3 body & 88,673 & 47,769 \\
Other 4 body & 206,255 & 110,333 \\
Other $5+$ body & $2,580,313$ & 465,627 \\
\hline Total & $10,429,063$ & $6,277,657$ \\
\hline
\end{tabular}

TABle B.5: Distribution of final states in high mass $B^{0} \rightarrow X_{s}^{0} \gamma$ signal MC data. The hadronic mass is determined by the $\mathrm{KN}$ model. 


\begin{tabular}{|l|l|l|}
\hline Mode & Weight & Hadronic Mass $\geq 1.0 \mathrm{GeV} / c^{2}$ \\
\hline$B^{0} \rightarrow K^{+} \pi^{-} \gamma(1)$ & $0.65 \pm 0.04$ & 631,378 \\
$B^{0} \rightarrow K^{+} \pi^{0} \gamma(2)$ & $0.36 \pm 0.06$ & 214,271 \\
$B^{0} \rightarrow K^{+} \pi^{-} \pi^{+} \gamma(3)$ & $1.34 \pm 0.11$ & $1,472,239$ \\
$B^{0} \rightarrow K^{+} \pi^{-} \pi^{0} \gamma(4)$ & $1.35 \pm 0.11$ & $1,801,317$ \\
$B^{0} \rightarrow K^{+} \pi^{-} \pi^{+} \pi^{-} \gamma(5)$ & $0.75 \pm 0.27$ & 295,271 \\
$B^{0} \rightarrow K^{+} \pi^{-} \pi^{+} \pi^{0} \gamma(6)$ & $1.00 \pm 0.23$ & $1,004,779$ \\
$B^{0} \rightarrow K^{+} \eta \gamma(7)$ & $1.05 \pm 0.41$ & 89,289 \\
2 body no $\pi^{0}$ & $0.50 \pm 0.07$ & 485,050 \\
2 body $1 \pi^{0}$ & $0.19 \pm 0.12$ & 112,903 \\
3 body no $\pi^{0}$ & $1.02 \pm 0.14$ & $1,126,119$ \\
3 body $1 \pi^{0}$ & $1.34 \pm 0.24$ & $1,784,915$ \\
4 body no $\pi^{0}$ & $2.67 \pm 0.96$ & $1,044,859$ \\
4 body $1 \pi^{0}$ & $1.29 \pm 0.61$ & $1,336,953$ \\
$3 / 4$ body $2 \pi^{0}$ & $1.89 \pm 1.33$ & $2,330,544$ \\
5 body & $1.32 \pm{ }_{-1.32}^{+1.55}$ & $2,994,966$ \\
$\eta$ modes & $0.83 \pm{ }_{-0.83}^{+1.00}$ & 358,122 \\
$K K K$ modes & $0.27 \pm{ }_{-0.27}^{+0.54}$ & 73,274 \\
Unclassified & 0.64 & $3,698,624$ \\
\hline Total & 1.00 & $20,854,463$ \\
\hline
\end{tabular}

TABLE B.6: Application of weights to high mass $B \rightarrow X_{s} \gamma$ signal MC data.

\begin{tabular}{|l|l|l|}
\hline Mode & $m_{X}^{\text {true }} \geq 1.0 \mathrm{GeV} / c^{2}$ & $1.0 \leq m_{X}^{\text {true }} \leq 2.0 \mathrm{GeV} / c^{2}$ \\
\hline$B^{+} \rightarrow \pi^{+} \pi^{0} \gamma(2)$ & 110,859 & 95,550 \\
$B^{+} \rightarrow \pi^{+} \pi^{-} \pi^{+} \gamma(3)$ & 95,291 & 79,024 \\
$B^{+} \rightarrow \pi^{+} \pi^{0} \pi^{0} \gamma(8)$ & 88,869 & 73,913 \\
$B^{+} \rightarrow \pi^{+} \pi^{-} \pi^{+} \pi^{0} \gamma(6)$ & 183,197 & 142,670 \\
$B^{+} \rightarrow \pi^{+} \eta \gamma(7)$ & 12,790 & 11,000 \\
Other 2 body & 9,907 & 6,985 \\
Other 3 body & 47,284 & 34,937 \\
Other 4 body & 87,150 & 58,688 \\
$5+$ body & 566,589 & 220,478 \\
\hline Total & $1,201,936$ & 723,245 \\
\hline
\end{tabular}

TABLE B.7: Distribution of final states in high mass $B^{+} \rightarrow X_{d}^{+} \gamma$ signal MC data. The hadronic mass is determined by the $\mathrm{KN}$ model. 


\begin{tabular}{|l|l|l|}
\hline Mode & $m_{X}^{\text {true }} \geq 1.0 \mathrm{GeV} / c^{2}$ & $1.0 \leq m_{X}^{\text {true }} \leq 2.0 \mathrm{GeV} / c^{2}$ \\
\hline$B^{0} \rightarrow \pi^{+} \pi^{-} \gamma(1)$ & 99,331 & 85,512 \\
$B^{0} \rightarrow \pi^{+} \pi^{-} \pi^{0} \gamma(4)$ & 197,465 & 165,434 \\
$B^{0} \rightarrow \pi^{+} \pi^{-} \pi^{+} \pi^{-} \gamma(6)$ & 44,327 & 32,459 \\
$B^{0} \rightarrow \pi^{+} \pi^{-} \pi^{0} \pi^{0} \gamma(9)$ & 131,267 & 101,180 \\
Other 2 body & 44,336 & 36,704 \\
Other 3 body & 53,659 & 39,488 \\
Other 4 body & 62,555 & 39,280 \\
$5+$ body & 568,768 & 222,774 \\
\hline Total & $1,201,708$ & 722,831 \\
\hline
\end{tabular}

TABLE B.8: Distribution of final states in high mass $B^{0} \rightarrow X_{d}^{0} \gamma$ signal MC data. The hadronic mass is determined by the KN model. 


\section{References}

[1] BABAR Collaboration, B. Aubert et al., "Measurement of $B \rightarrow X \gamma$ Decays and Determination of $\left|V_{t d} / V_{t s}\right|$," Phys. Rev. Lett. 102161803 (2009), BABAR Analysis Document \#1340.

[2] N. Cabibbo, "Unitary Symmetry and Leptonic Decays," Phys. Rev. Lett. 10531 (1963).

[3] M. Kobayashi and T. Maskawa, "CP Violation in the Renormalizable Theory of Weak Interactions," Prog. Theor. Phys. 49652 (1973).

[4] P. F. Harrison and H. R. Quinn (Ed.), "The BABAR Physics Book." Technical Report SLAC-R-504 (1998).

[5] A. Seiden, Particle Physics a Comprehensive Introduction, Addison Wesley (2005).

[6] D. Griffiths, Introduction to Elementary Particles, John Wiley \& Sons, Inc. (1987).

[7] F. Halzen and A. D. Martin, Quarks $\&$ Leptons: An Introductory Course in Modern Particle Physics, John Wiley \& Sons, Inc. (1984).

[8] N. Ellis and C. Dib (Ed.), "2007 CERN-CLAF School of High Energy Physics Proceedings," Technical Report CERN-2008-004 (2008).

[9] T. J. Greenshaw (Ed.), "Proceedings of the School for Experimental High Energy Physics Students held 3 to 15 September 2006," Technical Report RAL-TR-2007004 (2007).

[10] S. L. Glashow, "Partial Symmetries of Weak Interactions," Nucl. Phys. 22579 (1961).

[11] A. Salam and J. C. Ward, "Gauge Theory of Elementary Interactions," Phys. Rev. B136 763 (1964).

[12] S. Weinberg, "A Model of Leptons," Phys. Rev. Lett. 191264 (1967).

[13] P. W. Higgs, "Broken Symmetries, Massless Particles and Gauge Fields," Phys. Lett. 12132 (1964). 
[14] Particle Data Group, C. Amsler et al., "The Review of Particle Physics," Phys. Lett. B667 1 (2008).

[15] L. Wolfenstein, "Parametrization of the Kobayashi-Maskawa Matrix," Phys. Rev. Lett. 551945 (1983).

[16] A. J. Buras, M. E. Lautenbacher and G. Ostermaier, "Waiting for the top quark mass, $K^{+} \rightarrow \pi^{+} \nu \bar{\nu}, B_{s}^{0}-\bar{B}_{s}^{0}$ mixing and CP asymmetries in $B$ decays," Phys. Rev. D50 3433 (1994).

[17] S. Weinberg, The Quantum Theory of Fields, Cambridge University Press (1995).

[18] J. H. Christenson et al., "Evidence for the $2 \pi$ Decay of the $K_{2}^{0}$ Meson," Phys. Rev. Lett. 13138 (1964).

[19] BABAR Collaboration, B. Aubert et al., "Measurement of Time-Dependent $C P$ Asymmetry in $B^{0} \rightarrow c \bar{c} K^{(*) 0}$ Decays," Phys. Rev. D79 072009 (2009), BABAR Analysis Document \#1996.

[20] BABAR Collaboration, B. Aubert et al., "Observation of $C P$ Violation in $B^{0} \rightarrow$ $K^{+} \pi^{-}$and $B^{0} \rightarrow \pi^{+} \pi^{-}, "$ Phys. Rev. Lett. 99021603 (2007), BABAR Analysis Document \#1551.

[21] BABAR Collaboration, B. Aubert et al., "Evidence for Direct $C P$ Violation from Dalitz-plot analysis of $B^{ \pm} \rightarrow K^{ \pm} \pi^{\mp} \pi^{ \pm}$," Phys. Rev. D78 012004 (2008), BABAR Analysis Document \#1181.

[22] A. Ali, H. Asatrian and C. Greub, "Inclusive Decay Rate for $B \rightarrow X_{d}+\gamma$ in Nextto-Leading Logarithmic Order and CP Asymmetry in the Standard Model," Phys. Lett. B429 87 (1998).

[23] G. Buchalla, A. Buras and M. E. Lautenbacher, "Weak Decays Beyond Leading Logarithms," Rev. Mod. Phys. 681125 (1996).

[24] Tobias Hurth, "Present Status of Inclusive Rare B Decays," Rev. Mod. Phys. 75 1159 (2003).

[25] A. J. Buras, "Operator Product Expansion, Renormalisation Group and Weak Decays," Lect. Notes Phys. 55865 (2000).

[26] A. Ali, E. Lunghi, A. Ya. Parkhomenko, "Implication of the $B \rightarrow(\rho, \omega) \gamma$ Branching Ratios for the CKM Phenomenology," Phys. Lett. B595 323 (2004).

[27] A. L. Kagan and M. Neubert, "QCD Anatomy of $B \rightarrow X_{s} \gamma$ Decays," Eur. Phys. J. C7 5 (1999). 
[28] D. Benson, I.I. Bigi and N. Uraltsev, "On the Photon Energy Moments and their 'Bias' Corrections in $B \rightarrow X_{s} \gamma$," Nucl. Phys. B710 371 (2005).

[29] B.O. Lange, M. Neubert and G. Paz, "Theory of Inclusive Charmless B Decays and the Extraction of $V_{u b}, "$ Phys. Rev. D72 073006 (2005).

[30] BABAR Collaboration, B. Aubert et al., "Measurements of the $B \rightarrow X_{s} \gamma$ Branching Fraction and Photon Spectrum from a Sum of Exclusive Final States," Phys. Rev. D72 052004 (2005), BABAR Analysis Document \#768.

[31] O. L. Buchmüller and H. U. Flächer, "Fit to Moments of Inclusive $B \rightarrow X_{c} l \bar{\nu}$ and $B \rightarrow X_{s} \gamma$ Decay Distributions using Heavy Quark Expansions in the Kinetic Scheme," Phys. Rev. D73 073008 (2006).

[32] CDF Collaboration, A. Abulencia et al., "Observation of $B_{s}^{0}-\bar{B}_{s}^{0}$ Oscillations." Phys. Rev. Lett. 97242003 (2006).

[33] BABAR Collaboration, B. Aubert et al., "Measurements of Branching Fractions for $B^{+} \rightarrow \rho^{+} \gamma, B^{0} \rightarrow \rho^{0} \gamma$, and $B^{0} \rightarrow \omega \gamma . "$ Phys. Rev. D78 112001 (2008), BABAR Analysis Document \#1693.

[34] BELLE Collaboration, N. Taniguchi et al., "Measurement of branching fractions, isospin and $C P$-violating asymmetries for exclusive $b \rightarrow d \gamma$ modes." Phys. Rev. Lett. 101111801 (2008).

[35] BABAR Collaboration, D. Boutigny et al., "BABAR Technical Design Report." Technical Report SLAC-R-475 (1995).

[36] M. S. Zisman (Ed.), "PEP-II, An Asymmetric B Factory: Conceptual Design Report." Technical Report SLAC-R-418 (1993).

[37] S. Ecklund, C. Field and G. Mazaheri, "A Fast Luminosity Monitor System for PEP II," Nucl. Instrum. Meth. A463 68 (2001).

[38] BABAR Collaboration, B. Aubert et al., "The BABAR Detector." Nucl. Instrum. Meth. A479 1 (2002).

[39] BABAR Collaboration, Electron Identification Analysis Working Group, "Cut-Based Electron Identification," BABAR Analysis Document \#90 (2001).

[40] BABAR Collaboration, W. Menges, "The BABAR Muon System Upgrade." in "Proceedings of 2005 IEEE Nuclear Science Symposium and Medical Imaging Conference," (2005).

[41] Charge conjugation is implied throughout the subsequent text unless otherwise stated. 
[42] BABAR Collaboration, B. Aubert et al., "Measurement of the Branching Fraction and Photon Energy Moments of $B \rightarrow X_{s} \gamma$ and $A_{C P}\left(B \rightarrow X_{s+d} \gamma\right)$," Phys. Rev. Lett. 97171803 (2006), BABAR Analysis Document \#323.

[43] BABAR Collaboration, B. Aubert et al., "Measurement of the $B \rightarrow X_{s} \gamma$ Branching Fraction and Photon Energy Spectrum using the Recoil Method," Phys. Rev. D77 051103 (2008), BABAR Analysis Document \#1609.

[44] T. Sjöstrand, "High Energy Physics Event Genereation with PYTHIA 5.7 and JETSET 7.4," Comput. Phys. Commun. 8274 (1994).

[45] BABAR Collaboration, G. D. McGregor, "B Counting at BABAR," Technical Report SLAC-R-912 (2008).

[46] GEANT4 Collaboration, S. Agostinelli et al., "GEANT4: A Simulation Toolkit," Nucl. Instrum. Meth. A506 250 (2003).

[47] E. Barberio and Z. Was, "PHOTOS: A Univeral Monte Carlo for QED Radiative Corrections." Comput. Phys. Commun. 79291 (1994).

[48] BABAR Collaboration, G. Cowan et al., "Smearing of Monte Carlo Calorimeter Simulation," BABAR Analysis Document \#1639 (2007).

[49] BABAR Collaboration, M. Carpinelli et al., "The BABAR Vertexing," BABAR Analysis Document \#102 (2001).

[50] BABAR Collaboration, P. Ongmongkolkul et al., "Particle Identification Using Error Correction Output Code Multiclass Classifier," BABAR Analysis Document \#2199 (2009).

[51] BABAR Collaboration, W. T. Ford, "Choice of Kinematic Variables in $B$ Meson Reconstruction," BABAR Analysis Document \#53 (2000).

[52] E. Fahri, "Quantum Chromodynamics Test for Jets," Phys. Rev. Lett. 391587 (1977).

[53] G.C. Fox and S. Wolfram, "Observables for the Analysis of Event Shapes in $e^{+} e^{-}$ Annihilation and Other Processes," Phys. Rev. Lett. 411581 (1978).

[54] BABAR Collaboration, J. Ocariz et al., "Background Fighting in Charmless Twobody Analyses," BABAR Analysis Document \#346 (2002).

[55] J.D. Bjorken and S.J. Brodsky, "Statistical Model for Electron-Positron Annihilation into Hadrons," Phys. Rev. D1 1416 (1970). 
[56] BABAR Collaboration, J. Beringer, "BTagger - A Multivariate Tagging Algorithm with Categories Based on the Physics of the $B_{\text {tag }}$ Decay," BABAR Analysis Document \#317 (2002).

[57] BABAR Collaboration, D. Lange et al., "BtgTest and Tag04: Studies Towards an Improved Tagging Algorithm," BABAR Analysis Document \#730 (2004).

[58] BABAR Collaboration, R Bartoldus et al., "Trigger and Filter Documentation for the Run 1 Data," BABAR Analysis Document \#194 (2002).

[59] BABAR Collaboration, B. Aubert et al., "Branching Fractions of $B^{+} \rightarrow \rho^{+} \gamma, B^{0} \rightarrow$ $\rho^{0} \gamma$, and $B^{0} \rightarrow \omega \gamma$." Phys. Rev. Lett. 98151802 (2007), BABAR Analysis Document \#1301.

[60] I. Narsky, "StatPatternRecognition: A C ++ Package for Statistical Analysis of High Energy Physics Data," arXiv:physics/0507143 (2005).

[61] W. Verkerke and D. Kirkby, "The RooFit Toolkit for Data Modeling," arXiv:physics/0306116 (2003).

[62] F. James and M. Roos, "Minuit' A System for Function Minimization and Analysis of the Parameter Errors and Correlations," Comput. Phys. Commun. 10343 (1975).

[63] L. Lyons, Statistics for Nuclear and Particle Physicists, Cambridge University Press (1992).

[64] R. Barlow, Statistics: A Guide to the Use of Statistical Methods in the Physical Sciences, John Wiley \& Sons, Ltd. (1989).

[65] ARGUS Collaboration, H. Albrecht et al., "Reconstruction of B Mesons," Phys. Lett. B185 218 (1987).

[66] J.E. Gaiser, "Hadron Production by $e^{+} e^{-}$Annihilation at Center-of-Mass Energies Between 2.6 and 7.8 GeV," Ph.D Thesis, Technical Report SLAC-255 (1982).

[67] BABAR Collaboration, B. Aubert et al., "Measurement of the Branching Fraction and the Time-Dependent $C P$-Violating Asymmetry for the Decay $B^{0} \rightarrow K_{S}^{0} \pi^{0}$," Phys. Rev. D71 111102 (2005), BABAR Analysis Document \#904.

[68] BABAR Collaboration, I.M. Nugent, "Tau31 Tracking Efficiency Study for 2004," BABAR Analysis Document \#931 (2004).

[69] BABAR Collaboration, M. Allen et al., "A Measurement of $\pi^{0}$ Efficiency Using $\tau \rightarrow$ $\rho \nu$ and $\tau \rightarrow \pi \nu$ Decays," BABAR Analysis Document \#870 (2004). 
[70] BABAR Collaboration, D. Payne et al., "Single Photon Efficiency Using $\mu^{+} \mu^{-} \gamma$," BABAR Analysis Document \#1110 (2005).

[71] BABAR Collaboration, B. Aubert et al., "Measurement of $B \rightarrow K^{*} \gamma$ Branching Fractions and Charge Asymmetries," Phys. Rev. Lett. 88101805 (2002), BABAR Analysis Document \#33.

[72] BELLE Collaboration, A. Abashian et al., "The BELLE Detector," Nucl. Instrum. Meth. A479 117 (2002).

[73] LHCb Collaboration, A. Augusto Alves et al., "The LHCb Detector at the LHC," JINST 3 S08005 (2008).

[74] LHCb Collaboration, G. Pakhlova and I. Belyaev, "Radiative B Decays with LHCb," Technical Report LHCb 2003-090 (2003).

[75] SuperB Collaboration, M. Bona et al., "SuperB: A High-Luminosity Asymmetric e+ e- Super Flavor Factory. Conceptual Design Report," arXiv:0709.0451 [hep-ex] (2007).

[76] BELLE II Collaboration, I. Adachi et al., "sBelle Design Study Report," arXiv:0810.4084 [hep-ex] (2008).

[77] BABAR Collaboration, P. del Amo Sanchez et al., "Study of $B \rightarrow X \gamma$ Decays and Determination of $\left|V_{t d} / V_{t s}\right| . "$ arXiv:1005.4087 [hep-ex] (2010). 
"We do not yet know all the basic laws: there is an expanding frontier of ignorance."

Richard Feynman 